\title{
Superconformal Invariants and Correlation Functions
}

\author{
Dissertation \\ zur Erlangung des \\ mathematisch-naturwissenschaftlichen Doktorgrades \\ „,Doctor rerum naturalium” \\ der Georg-August-Universität Göttingen
}

vorgelegt von

Holger Knuth

aus Bergisch-Gladbach

Göttingen, 2012 
Referent: Prof. Dr. Karl-Henning Rehren

Korreferentin: Prof. Laura Covi, $\mathrm{PhD}$

Tag der mündlichen Prüfung: 16. April 2012 


\section{Contents}

\begin{tabular}{lll}
\hline & Introduction & 1
\end{tabular}

\begin{tabular}{llr}
\hline 2 & Global conformal field theory & $\mathbf{7}$
\end{tabular}

2.1 Wightman axioms . . . . . . . . . . . . . . . . . 8

2.2 GCI and rationality of correlation functions . . . . . . . . . . 9

2.3 Partial wave expansion . . . . . . . . . . . . . . . . . . . . 11

$2.4 \quad$ Wightman positivity $\ldots \ldots \ldots \ldots \ldots$

$\begin{array}{lll}3 \mathcal{N}=1 \text { Superconformal field theories } & \mathbf{1 5}\end{array}$

3.1 Super-Poincaré algebra . . . . . . . . . . . . . . . . . . 16

3.2 Superspace . . . . . . . . . . . . . . . . . . . . . . . . 17

3.3 Superconformal symmetry . . . . . . . . . . . . . . . . . . . . . . . . 19

3.4 SUSY and superconformal field representations . . . . . . . . . . 25

$3.4 .1 \quad$ Supersymmetry multiplets . . . . . . . . . . . . . . . 26

3.4 .2 Superconformal multiplets . . . . . . . . . . . . . . . . . . 28

3.4 .3 Superfields $\ldots \ldots \ldots \ldots \ldots \ldots$

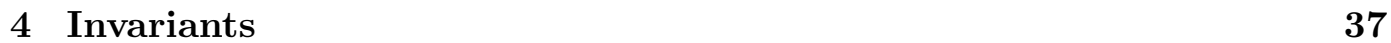

4.1 Three-point invariants . . . . . . . . . . . . . . . . . . . 38

4.2 Non-nilpotent four-point invariants . . . . . . . . . . . . . . . . . 40

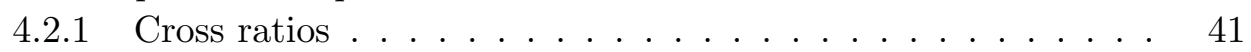

4.2 .2 Other non-nilpotent invariants . . . . . . . . . . . . . 42

4.3 Nilpotent four-point invariants $\ldots \ldots \ldots$. . . . . . . . . . . . 46

$4.3 .1 \quad$ Construction $\ldots \ldots \ldots \ldots \ldots$. . . . . . . . . . . . . . . . . 47

$4.3 .2 \quad$ Monomials of nilpotent invariants . . . . . . . . . . . . 54

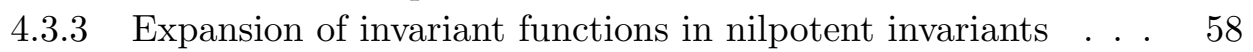

$4.3 .4 \quad$ Algorithmic calculations with explicit expansions of cross ratios and other functions of superspace coordinates . . . . . 65

$4.3 .5 \quad$ Alternative nilpotent invariants . . . . . . . . . . . 71

$\begin{array}{llr}5 & \text { Correlation functions } & \mathbf{7 5}\end{array}$

5.1 The two-point function . . . . . . . . . . . . . . . . 77

5.2 The three-point function . . . . . . . . . . . . . . . . . . . 78

5.3 The four-point function $\ldots \ldots \ldots \ldots$. . . . . . . . . . . 81

5.3 .1 Lowest Graßmannian order . . . . . . . . . . . . . . . . . 84 
5.3 .2 Iterative conclusion to higher Graßmannian orders . . . . . . 88

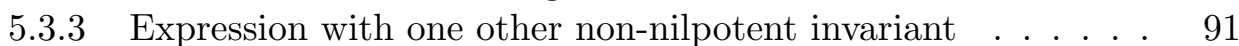

5.3 .4 Rational four-point functions $\ldots \ldots \ldots . \ldots . \ldots 93$

\begin{tabular}{|lll}
\hline 6 & From conformal to superconformal & 97
\end{tabular}

6.1 Pole bounds . . . . . . . . . . . . . . . . . . . . . 9 97

6.2 Partial wave expansion $\ldots \ldots \ldots \ldots$. . . . . . . . . . . . . 102

\begin{tabular}{lll}
\hline 7 & Conclusion & 105
\end{tabular}

\begin{tabular}{|lr}
\hline A Conventions and useful equations & 107
\end{tabular}

A.1 Pauli matrices . . . . . . . . . . . . . . . . . . . . . . . 107

A.2 Superconformal intervals . . . . . . . . . . . . . . . . . . . . 109

\begin{tabular}{ll}
\hline $\mathcal{N}=1$ superconformal algebra & 111
\end{tabular}

\begin{tabular}{lr}
\hline C Details on Invariants & 115
\end{tabular}

C.1 Lowest order of the nilpotent invariants $I_{i j k}$ and their derivatives . . 115

C.2 Derivatives of $T_{111}, T_{212}, T_{222}$ and $T_{0} \ldots \ldots \ldots \ldots \ldots 117$

C.3 Prod. of nilpotent invariants and their derivatives . . . . . . . . . 118

C.3.1 Proof of Lemma $5.3 \ldots \ldots$. . . . . . . . . . . . . . . . . . 119

C.3.2 Proof of Lemmal5.4 . . . . . . . . . . . . . . . . . . . . . 122

C.4 R-symmetry violating partial four-point invariants . . . . . . . . . 123

\begin{tabular}{lr}
\hline D Calculations using Maple & 129
\end{tabular}

D.1 Procedures for the calculation of expansions . . . . . . . . . . . . . 129

D.2 Replacing $\hat{X}_{1(2)-}^{2}$ by other non-nilpotent invariants . . . . . . . . . 133

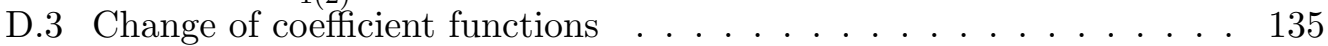

D.4 Expansion of inverses and square roots of Inv. . . . . . . . . . . . . 137

D.5 Combinatorics of non-nilpotent invariants . . . . . . . . . . . 137

\begin{tabular}{ll}
\hline Bibliography & 143
\end{tabular}

\begin{tabular}{ll}
\hline Acknowledgements & 149
\end{tabular} 


\section{Chapter 1}

\section{Introduction}

Quantum field theory has developed from the first steps it took as the straightforward application of 1920s quantum theory methods on classical fields to the modern picture of fields as elementary constituents in nature, but still remained a mystery regarding important conceptual questions. While the standard model of particle physics is a renormalizable quantum field theory, which produces extremely accurate and experimentally verified predictions for high energy physics, the perturbative techniques used for these predictions resemble a collection of recipes lacking a significant amount of mathematical understanding of the appearing entities. Already the appearance of non-linear functions of distributions does not rely on well-posed definitions.

On the one hand we are fortunate, that this situation, persisting for several decades now, allows the ignorance of the mathematical problems of quantum field theory for physicists interested in detailed results for high energy phenomenology. This has made the rapid progress in this field possible.

On the other hand this is at the same time a misfortune, as in many modern quantum field theory books students are not informed about and thus not sensitized for these problems.

This thesis is set up in the conceptual framework of axiomatic quantum field theory, which tries to construct quantum field theories within a consistent mathematical framework described by a set of axioms.

Axiomatic Quantum Field Theory. Although Hilbert published his mathematical problems [Hil] before all the groundbreaking developments of quantum physics and relativity in the early twentieth century, his text on the sixth problem could not match better the task axiomatic quantum field theory is concerned with. Framed by the reference to the situation at that time the central sentence is timeless ${ }^{1}$

The investigations on the foundations of geometry suggest the problem: To treat in the same manner, by means of axioms, those physical sciences in which mathematics plays an important part; in the first rank are the theory of probabilities and mechanic.

\footnotetext{
${ }^{1}$ This and the following two quotation originate form Hil].
} 
In his following description of the roles of mathematicians and physicists, one can perhaps recognize an ideal situation of a symbiosis of a mathematical physicist dealing with axiomatic quantum field theory and a phenomenologically interested quantum field theorist. Hilbert writes:

The mathematician will have also to take account not only of those theories coming near to reality, but also [...] of all logically possible theories. He must be always alert to obtain a complete survey of all conclusions derivable from the system of axioms assumed.

In axiomatic quantum field theory the systems of axioms try to express certain basic properties, which realistic theories should have. Still specific models investigated most often have unrealistic symmetries or dimensions of space-time. Although these choices are made because of the relative simplicity of these theories and not for reasons of completeness, they match the idea, that also unrealistic models within a realistic framework promote our view on quantum field theory.

The discussion of the sixth problem in [Hil] ends with

Further, the mathematician has the duty to test exactly in each instance whether the new axioms are compatible with the previous ones. The physicist, as his theories develop, often finds himself forced by the results of his experiments to make new hypotheses, while he depends, with respect to the compatibility of the new hypotheses with the old axioms, solely upon these experiments or upon a certain physical intuition, a practice which in the rigorously logical building up of a theory is not admissible. The desired proof of the compatibility of all assumptions seems to me also of importance, because the effort to obtain such proof always forces us most effectually to an exact formulation of the axioms.

Unfortunately this ideal symbiosis is not reached in quantum field theory, as we have already mentioned above.

While the interaction picture is used widely in perturbative calculations in quantum field theory, it is shown by Haag's theorem (cf. [Haa55, EF06]) not to exist, if one rigorously arguments with general properties of quantum field theories. Because of the empirical successes of perturbation theory this rigorous result is ignored by most quantum field theorists. The reason, why and under which limitations perturbation theory using the interaction picture, which has to be seen as a tool for calculations in quantum field theory, leads to correct results, remains unknown.

The Wightman axioms for correlation functions on Minkowski space in the fifties (published in the sixties [SW]) and later the Osterwalder-Schrader axioms for Euclidean Green's functions in the seventies OS73 took a central place in axiomatic quantum field theory. They collect the properties coming from the physical intuition, which a quantum field theory resp. equivalent problems in probability theory should have on the level of their correlation functions resp. their Schwinger functions.

With his axioms Wightman wanted to tackle, the "Main Problem of quantum field theory", as it is called in the book $[\mathrm{SW}]$ : 
$[\ldots]$ either to show that the idealizations involved in the fundamental notions of the theory (relativistic invariance, quantum mechanics, local fields, etc.) are incompatible in some physical sense, or to recast the theory in such a form that it provides a practical language for the description of elementary particle dynamics.

Already there it has been seen, that the Wightman axioms are consistent, as they are satisfied in free field theories. In a review of the situation in [FRS07 the authors point towards the second possibility of the "Main problem", when the investigations within different axiomatic approaches to quantum field theory including the Wightman axioms are evaluated:

None of them indicates a necessary failure or inconsistency of the framework of QFT.

Also in their book Wightman and Streater declared the goal to show, that there is an interacting theory satisfying his set of axioms. While this has been successful in low space-time dimensions, a four-dimensional interacting quantum field theory waits to be constructed. This would be a great step on the way to the long-term goal of a rigorous mathematical basis of gauge theories, which nature is obviously governed by. For the latter, however, it is known, that in general gauge theories lack a Hilbert space, which is an essential ingredient of the Wightman axioms. To present knowledge in Coulomb gauge the chance survives with a canonical quantization on a Hilbert space found for this case.

An important result within the Wightman approach is the Wightman reconstruction theorem. It shows, that the field representations within a theory are determined up to unitary equivalence by the collection of all Wightman (correlation) functions in this theory. This means, that this collection carries all information on this theory.

In chapter 2 the Wightman axioms and results from global conformal theories are reviewed. These theories are a good candidate to achieve the goal of an interaction four-dimensional quantum field theory.

The aim of the research project, which constitutes this thesis, is to enlarge the group of candidates with certain expedient properties to supersymmetric theories with global conformal symmetry.

One of these properties is the possibility to use a conformal partial wave expansion. In the conformal case and - in the consequence of this thesis - also the superconformal case this partial wave expansion leads to simple conditions equivalent to the positivity conditions for these correlation functions. This condition is one of the Wightman axioms needed for the probability interpretation of the correlation functions. It is extremely difficult to test, also because this has to be done for all n-point correlation functions.

Superconformal Symmetry. In the early beginnings of supersymmetry WZ74, MPS76, AMS76, AMS77, AMS78, four-dimensional $\mathcal{N}=1$ superconformal field theories came into the focus of several researchers. But due to the mainly perturbative approach to supersymmetric field theories, in which non-trivial theories could not sustain conformal invariance, the mainstream attached to super-Poincaré symmetry. 
Still insights in the conformal invariance of supersymmetric non-Abelian gauge theories at renormalization group fixed points with vanishing $\beta$-function (Sei95] and more recently [IW03, IW04, BIWW05]) brought new interest to a non-perturbative analysis of superconformal field theories in the mid-90s. The very recent study of new supergravity models with the potential to resolve some problems of minimal supersymmetric standard models $\left[\mathrm{FKL}^{+} 10 \mathrm{a}, \mathrm{FKL}^{+} 10 \mathrm{~b}\right]$ could possibly again renew attention on this topic. Here big emphasis is given to the underlying superconformal symmetry.

Chiral superfields gathered most attention not only because of their prominent role in supersymmetry through the construction of F-Term Lagrangians and as suitable multiplets containing elementary particles, but also for the sake of their relative simplicity. This thesis focuses on chiral and anti-chiral superfields as well, as it comes to correlation functions.

In the eighties the two authors of [DP85a, DP85b, DP87] concentrated on a group theoretical approach to extended conformal supersymmetry.

A later series of papers started also with results for chiral superfield in $\mathcal{N}=1$ HW99, HW97, HW96b, but it focused afterwards the analysis of theories with extended superconformal symmetry on analytic superspace (e.g. HW96a, HSW98, EHSW00, $\mathrm{EHP}^{+} 00, \mathrm{HH} 03, \mathrm{HH} 04$ ). This approach is purely on-shell. It was shown, that there are no nilpotent invariants of up to four points of analytic superspace [EHW99, HSSW00]. This is enough to investigate chiral/anti-chiral four-point functions, but cuts the perspective to deal with more general superfields.

Another line of work concentrated first on correlation functions of only chiral superfields for $\mathcal{N}=1$ Osb99, DO01a]. While the two point function is a pure contact term, which does not fulfill the Wightman axioms, the three point function is only consistent with the Ward identities, if the total R-charge is one. These publications already introduce part of the notation, which we also use in this thesis. Later papers then mainly dealt with $\mathcal{N}=4$ [AEPS02, DO02, DO03, DO04, DO06].

A more general approach closely related to [Osb99] is found in the work of Park Par98, Par99. He has derived implications of superconformal symmetry on correlation functions of arbitrary quasi-primary fields. For that purpose all invariants of the $\mathcal{N}$-extended superconformal group for all $\mathcal{N} \geq 1$ were constructed on ordinary super Minkowski space. It is stated, that correlation functions with vanishing Rcharge are functions of these invariants. For the case of non-vanishing R-charge the correlation functions are nilpotent and depend on a larger set of invariants, which are not required to be invariant under the R-symmetry.

In chapter 3 we review the basics of supersymmetry and superspace, which we see as a tool to organize fields of Minkowski space related by supersymmetry within one superfield on superspace. Then the superconformal group is discussed following mainly the work in [Par99]. We also look at the field representation of supersymmetry and superconformal symmetry as multiplets on Minkowski space and as superfields on superspace.

Chapter 4 investigates the three- and four-point invariants for $\mathcal{N}=1$ from [Par99]. It is argued, that there are only 10 independent invariants of four points: two cross ratios and eight nilpotent invariants. This is an important result in this 
thesis because it shows, that in $\mathcal{N}=1$ superconformal symmetry fewer invariants are needed to express any superconformal four-point function with vanishing R-charge than thought hitherto ( Par99] argues it to be maximally 16). With this result we find all monomials of nilpotent invariants, which are independent in a way, so that we have a unique expansion for every four-point invariants in these nilpotent invariants. We also proof the uniqueness within theorem 4.1. At last we go into the technical details, so that we are able to change to alternative sets of four-point invariants needed in the following chapter.

The correlation functions of superconformal chiral and anti-chiral scalar superfields are discussed in chapter 5. Two- and three-point functions do not need a long discussion. We mainly state existing results for vanishing and non-vanishing R-charge. The discussion of four-point functions of chiral and anti-chiral scalar superfields, which is given only for vanishing R-charge, is arranged around the proof of theorem 5.1 giving a specific form especially suitable for the transfer of results from global conformal to global superconformal theories, but valid for all superconformal theories. In this proof we start from the general form of scalar four-point functions and look at the chiral and anti-chiral restriction imposed on this form. This gives us insight in the cancellation of terms from different invariants. The nilpotent invariants appear in a differential operator, which is applied in the proven form of the superconformal four-point function to the invariant function appearing in the conformal four-point function of the lowest component fields of the chiral and antichiral superfields. After this proof we treat the special case of rational four-point functions. This rationality is a consequence of global (super)conformal symmetry.

Chapter 6 finally gives the first results and the outlook on the transfer of results from global conformal to global superconformal four-point functions. In the first part pole bounds are investigated and in the second part the partial wave expansion is discussed.

In the appendix we have chapters containing the conventional choices and useful equations, appendix $\mathrm{A}$, the superconformal algebra, appendix $\mathrm{B}$, details of the invariants, which are needed in the main text, appendix C, and useful Maple procedures for calculations with invariants, appendix $\mathrm{D}$. The discussion of superconformal four-point invariants without R-symmetry, which is not needed for the rest of the thesis, can be found in appendix C.4. 


\title{
Chapter 2
}

\section{Global conformal field theory}

\begin{abstract}
After more than eighty years of work on quantum field theory, we look back on an enormously successful story, which lead to a standard model of particle physics with stunningly precise matches of theoretical calculations and experimental results. Still there are significant mathematical problems left to reach a status of the theory as reached in quantum mechanics, where one can speak of a mathematically complete and consistent formalism. A better mathematical understanding of QFT could lead us to non-perturbative results, which we still lack in most situations. This makes the efforts to rigorously construct a four-dimensional interacting quantum field theory and to show, that it fulfills certain axioms implied by the physical interpretation of the theory, especially worthwhile - even if it is reached in a simplified toy-model at first.
\end{abstract}

One of the consequences from physical interpretation, which has taken center stage, is the needed positivity of the inner product of the Hilbert space of states, which persistently resists its verification because of the complicated properties of products of fields in interacting theories. This positivity goes back to the probability interpretation of such inner products appearing as correlation functions in QFT. Consequently one needs the positivity of all correlation functions and the compliance of Schwarz inequalities of correlation functions. This is one of the Wightman axioms, which are the most prominent set of axioms for QFT. ${ }^{1}$

Conformal field theory in higher (than two) dimensions got into the focus of a constructive approach on the basis of the Wightman axioms in the past decade. It investigates these theories on a compactified space-time, on which the covariance of the fields being conformal representations on Minkowski space - and not a covering space - is postulated, leading to the global conformal invariance (GCI) of the Wightman function, the correlation functions in this framework. Here the focus lies on the construction of a non-trivial quantum field theory in four dimensions on the compactification of Minkowski space, $\mathbb{R}^{1,3}$. The rationality of the correlation functions has been proven and bilocal fields made a transfer of methods from two dimensional conformal field theories possible. Concerning the positivity axiom conformal partial

\footnotetext{
${ }^{1}$ In the last 50 years investigations starting from suitable sets of axioms - name giving to the field of axiomatic quantum field theory - led to significant results. A quite recent status report can be found in FRS07.
} 
wave analysis has provided an access for its validation with explicit results for GCI four-point functions.

In this chapter we first take a short look at the Wightman axioms and then summarize results for GCI field theories focussing the partial wave expansion and its role for the investigation of Wightman positivity.

\subsection{Wightman axioms}

The book SW published in 1964 contained Wightman's set of axioms in a form, which is the basis of work on the construction of quantum field theories on Minkowski space down to the present date. It combines the focus on the correlation functions as actual observables with expectations for a physically realistic local quantum field theory. These correlation functions are given as tempered distributions and the axioms are given as properties of these so-called Wightman functions evaluated at arbitrary test functions from Schwartz space.

Here we leave this more general frame and look at Wightman functions given as vacuum expectation values of products of fields. Consequently we state Wightman axioms on these fields, which are defined as operator valued distributions. These axioms are not more fundamental, as one could think at first, because the Wightman reconstruction theorem states, that up to unitary equivalence the theory is determined by its Wightman functions SW].

We state the axioms for a space-time $M$ together with a space-time symmetry group, $G$. This may be Poincaré symmetry on Minkowski space, $\mathbb{R}^{1,3}$ as in [JO00] or conformal symmetry on conformally compactified Minkowski space as reviewed in Wag. Here we leave these entities undetermined:

- Hilbert space and symmetry group: The space-time symmetry group, $G$, has a unitary representation $U$ acting on the state space, $\mathcal{H}$, a separable Hilbert space. It leaves an element $\Omega \in \mathcal{H}$, the vacuum vector, invariant. It is unique up to a factor. The commuting generators of the translations have joint spectrum in the closure of the forward lightcone.

- Covariant fields: The fields, $\phi_{i}$, as well as its hermitian conjugate, are operator valued distributions. A dense subset, $\mathcal{D}_{\mathcal{H}}$, of $\mathcal{H}$ is spanned by these fields $\phi_{i} f, f \in S(M)$, acting on the vacuum, $\Omega$. They transform under $g \in G$ as

$$
U(g) \phi(f) U(g)^{-1}=\omega(g) \phi\left(f \circ g^{-1}\right),
$$

where $\omega(g)$ is a finite dimensional representation of $g$.

- Locality: Two fields, $\phi(f)$ and $\chi(g)$, commute resp. anti-commute depending on their spin, if the support of $f$ and the support of $g$ are space-like separated.

- Completeness: Every subset of $\mathcal{D}_{\mathcal{H}}$, which is invariant under the action of the fields, is also dense in $\mathcal{H}$. 
When one wants to make contact between these axioms and known realistic quantum field theories, the problem starts right in the first sentence in combination with the second item: it is far from obvious, that there is a separable Hilbert space spanned by the fields. The positive definiteness of the inner product on $\mathcal{H}$ and so the positivity of all Wightman functions has to be tested. Therefore we look at the properties of the Wightman functions resulting from the above axioms. We have the n-point Wightman functions evaluated at n-point of $M$ :

$$
\mathcal{W}_{n}\left(x_{1}, \ldots, x_{n}\right)=\left\langle\Omega, \phi_{1}\left(x_{1}\right) \ldots \phi_{n}\left(x_{n}\right) \Omega\right\rangle=\left\langle\phi_{1}\left(x_{1}\right) \ldots \phi_{n}\left(x_{n}\right)\right\rangle .
$$

- Invariance: Because of the covariance of the fields and the invariance of the vacuum, the Wightman functions are invariant with respect to space-time transformations, $g \in G$ :

$$
\mathcal{W}_{n}^{\prime}=\bigotimes_{n} \omega(g) \mathcal{W}_{n} \circ g^{-1}=\mathcal{W}_{n}
$$

- Locality: For space-like separated points $x_{i}$ and $x_{i+1}, i \in\{1, \ldots, n\}$ we have

$$
\mathcal{W}_{n}\left(x_{1}, \ldots, x_{i}, x_{i+1}, \ldots, x_{n}\right)=\mathcal{W}_{n}\left(x_{1}, \ldots, x_{i+1}, x_{i}, \ldots, x_{n}\right)
$$

- Wightman-positivity: From the positivity of the norm of the Hilbert space follows the condition, that for all finite sequences of test functions, $f_{p}$,

$$
\begin{aligned}
& \sum_{p, q} \int_{M^{p+q}} \bar{f}\left(x_{p}, \ldots, x_{1}\right)\left\langle\phi_{1}^{*}\left(x_{1}\right) \ldots \phi_{p}^{*}\left(x_{p}\right) \phi_{p+1}\left(x_{p+1}\right) \ldots \phi_{p+q}\left(x_{p+q}\right)\right\rangle \\
& \cdot f\left(x_{p+1}, \ldots, x_{p+q}\right) \geq 0 .
\end{aligned}
$$

- Spectral condition: As the Wightman functions are translation invariant, they are functions of the differences, $x_{i}-x_{i+1}, i \in\{1 \ldots n-1\}$, The Fourier transformation of the Wightman functions with respect to these variables has its support in the $\mathrm{n}$-fold tensor product of the completion of the forward cone as a consequence of the spectrum of the translation generator.

Especially the Wightman-positivity is a crucial property, which has to be shown, when a quantum field theory is constructed according to the other properties.

\subsection{Global conformal invariance and rationality of cor- relation functions}

Global conformal invariance is the invariance of the correlation functions under a single-valued action of the fourfold cover $G=S U(2,2)$ of the conformal group whenever $x$ and $g x, g \in G$, both belong to Minkowski space. This definition is stated in NST02a.

For conformal symmetry we have in eq. (2.1.1) a cocycle $\omega(g, x)$ instead of $\omega(g)$. Global conformal invariance implies a much stronger locality condition as the one 
of the Wightman axioms. As a pair of time-like separated points can be mapped by conformal transformations into space-like separated points, the Huygens' principle holds. Two fields evaluated at two points, which are not light-like separated, commute resp. anti-commute.

The field representations, being unitary representations of $S U(2,2)$, are labeled following [Mac77] by the scaling dimension, $d \in \mathbb{R}$, and $\left(j_{1}, j_{2}\right)$, the labels of a finite dimensional irreducible representation of $S L(2, \mathbb{C})$ (specifically on Minkowski space). These representations are induced from the ones of the compact subgroup $S(U(2) \times U(2))$ and their scaling dimension has a lower limit depending on $\left(j_{1}, j_{2}\right)$ :

$$
j_{1}=0 \vee j_{2}=0 \Rightarrow d \geq j_{1}+j_{2}+1 \quad, \quad j_{1} \neq 0 \wedge j_{1} \neq 0 \Rightarrow d \geq j_{1}+j_{2}+2 .
$$

In [NT01] it has been shown, that global conformal invariance, locality, translation invariance and spectral conditions imply, that all Wightman functions are rational functions:

$$
\mathcal{W}_{n}\left(x_{1}, \ldots, x_{n}\right)=\mathcal{P}\left(x_{1}, \ldots, x_{n}\right) \prod_{1 \leq k<l \leq n}\left[\left(x_{k}-x_{l}\right)^{2}+\mathrm{i} \epsilon\left(x_{k}^{0}-x_{l}^{0}\right)\right]^{-\mu_{k l}},
$$

where the pole degrees, $\mu_{k l}$, have an upper bound, which only depends on the two fields, $\phi_{k}$ and $\phi_{l}$, belonging to the representations $\left(d_{k}, j_{1 k}, j_{2 k}\right)$ and $\left(d_{l}, j_{1 l}, j_{2 l}\right)$, respectively:

$$
\mu_{k l} \leq \llbracket \frac{d_{k}+j_{1 k}+j_{2 k}+d_{l}+j_{1 l}+j_{2 l}}{2}-\frac{1-\delta_{j_{1 k} j_{2 l}} \delta_{j_{2 k} j_{1 l}} \delta_{d_{k} d_{l}}}{2} \rrbracket,
$$

where, for $r \in \mathbb{R}, n=[r] \in \mathbb{Z}$ is the number, such that $n \leq r$ and $n+1 \geq r$.

The highest pole contributing here is the one of the two point function, if it does not vanish anyway. This is just the cause of the Kronecker deltas. If the charges are not identical and thus the two point function is zero, the corresponding pole bound of the four-point function is equal to the one of the truncated part of this correlation function .

In the case of two fields with identical charges, the truncated four-point function has in general a stronger bound for these two fields than the whole four-point function, which contains the two point function of these fields(cf. [NT01]). Hence the bound of the truncated four-point function is generally eq. 2.2.3 without the Kronecker deltas. For identical charge we then get:

$$
\begin{aligned}
\left\langle\ldots \psi^{*}\left(x_{i}\right) \ldots \psi\left(x_{j}\right) \ldots\right\rangle^{T} & \sim\left[\left(x_{i}-x_{j}\right)^{2}+\mathrm{i} \epsilon\left(x_{i}^{0}-x_{j}^{0}\right)\right]^{-\mu_{i j}} \\
\text { with } \quad \mu_{i j} & \leq \llbracket d+j_{1}+j_{2}-\frac{1}{2} \rrbracket=d+j_{1}+j_{2}-1 .
\end{aligned}
$$

Here the field $\psi$ has the quantum numbers $\left(d, j_{1}, j_{2}\right)$ and the $\sim$ here means, that the whole function looks like eq. (2.2.2).

For real scalar fields belonging to the representations $(d, 0,0)$ these pole bounds get especially simple. For the truncated four-point function of four such fields with 
the same scaling dimension, $d$, are given in [NST02a]:

$$
\begin{aligned}
\mathcal{W}_{s c ., 4}^{T}(d)\left(x_{1}, \ldots, x_{4}\right)= & \mathcal{W}_{s c ., 4}\left(x_{1}, \ldots, x_{4}\right)-\mathcal{W}_{s c ., 2}\left(x_{1}, x_{2}\right) \mathcal{W}_{s c ., 2}\left(x_{3}, x_{4}\right) \\
& -\mathcal{W}_{s c ., 2}\left(x_{1}, x_{3}\right) \mathcal{W}_{s c ., 2}\left(x_{2}, x_{4}\right)-\mathcal{W}_{s c ., 2}\left(x_{1}, x_{4}\right) \mathcal{W}_{s c ., 2}\left(x_{2}, x_{3}\right) \\
= & \frac{\left[\left(x_{1}-x_{3}\right)^{2}\left(x_{2}-x_{4}\right)^{2}\right]^{d-2}}{\left[\left(x_{1}-x_{2}\right)^{2}\left(x_{2}-x_{3}\right)^{2}\left(x_{3}-x_{4}\right)^{2}\left(x_{1}-x_{4}\right)^{2}\right]^{d-1}} \sum_{\substack{i, j \geq 0 \\
i+j \leq 2 d-3}} c_{i j} s^{i} t^{j}
\end{aligned}
$$

with

$$
s=\frac{\left(x_{1}-x_{2}\right)^{2}\left(x_{3}-x_{4}\right)^{2}}{\left(x_{1}-x_{3}\right)^{2}\left(x_{2}-x_{4}\right)^{2}} \quad, \quad t=\frac{\left(x_{1}-x_{4}\right)^{2}\left(x_{2}-x_{3}\right)^{2}}{\left(x_{1}-x_{3}\right)^{2}\left(x_{2}-x_{4}\right)^{2}} .
$$

But only $\llbracket d^{2} / 3 \rrbracket$ coefficients are independent because of the locality properties of this Wightman function:

$$
c_{i j}=c_{j i} \quad \text { and } \quad c_{i j}=c_{i(2 d-3-i-j)}=c_{(2 d-3-i-j) j},
$$

which leaves $c_{i j}$ with $i \leq j \leq \frac{2 d-3-i}{2}$.

In the next section we will look at the partial wave expansion and show, how the coefficients of this expansion can be related to the finite number of independent amplitudes, which are left after the evaluation of pole bounds and locality properties for identical fields in the four-point function.

\subsection{Partial wave expansion}

The analysis of bilocal fields brought deeper insight into the structure of the Wightman functions. These fields, $V_{l}\left(x_{1}, x_{2}\right)$, appear in an operator product expansion of two neutral scalar fields:

$$
\begin{aligned}
\phi_{1}\left(x_{1}\right) \phi_{2}\left(x_{2}\right)= & \mathcal{W}_{s c ., 2}\left(x_{1}, x_{2}\right)+\sum_{l=1}^{d-1}\left(\frac{1}{4 \pi\left[\left(x_{1}-x_{2}\right)^{2}+\mathrm{i} \epsilon\left(x_{1}^{0}-x_{2}^{0}\right)\right]}\right)^{d-l} V_{l}\left(x_{1}, x_{2}\right) \\
& +: \phi_{1}\left(x_{1}\right) \phi_{2}\left(x_{2}\right):
\end{aligned}
$$

This splits the operator product into the different twist contributions, which is the difference of dimension and rank. In [NST02a] the main focus lies on scalar fields with scaling dimension 2. Based on that publication it has been shown in [NRT08, that models of these fields can be reduced to free fields. In [NST02b] the authors started to look at $d=4$ fields with the possible interpretation as a gauge invariant Lagrangian in mind. The next paper, [NST03], has led to another possibility to write down the truncated four point Wightman function, eq. (2.2.5), for $d=4$. This contains different five independent coefficients and has been the starting point for the example of the partial wave expansion of these correlation functions detailed in NRT05. 
The partial wave expansion of a four-point function is defined by the insertion of projectors, $\Pi_{k, L}$, which project onto each symmetric traceless representation $(2 k+$ $L, L / 2, L / 2)$ and, of course, form a partition of the unit:

$$
\begin{aligned}
\mathcal{W}_{s c ., 4}(4)\left(x_{1}, \ldots, x_{4}\right) & =\left\langle\phi\left(x_{1}\right) \phi\left(x_{2}\right) \phi\left(x_{3}\right) \phi\left(x_{4}\right)\right\rangle \\
& =\sum_{k, L}\left\langle\phi\left(x_{1}\right) \phi\left(x_{2}\right) \Pi_{k, L} \phi\left(x_{3}\right) \phi\left(x_{4}\right)\right\rangle \\
& =\left\langle\phi\left(x_{1}\right) \phi\left(x_{2}\right)\right\rangle\left\langle\phi\left(x_{3}\right) \phi\left(x_{4}\right)\right\rangle \sum_{k, L} B_{k, L} \beta_{k, L}(s, t) .
\end{aligned}
$$

For a four-point function of identical fields, the sum only contains the vacuum contribution, $k=L=0$, all positive even twists, $2 k$, and all non-negative even spins $L$ (cf. [NST03, NRT05]).

With DO01b] we get the partial waves, $\beta_{k, L}$, as functions of chiral variables, $u$ and $v$, defined by $u v=s$ and $(1-u)(1-v)=t$ :

$$
\beta_{k, L}=\frac{u v}{u-v}\left(G_{k+L}(u) G_{k-1}(v)-G_{k+L}(v) G_{k-1}(u)\right),
$$

where $G_{n}(z)$ is the product of a monomial of its argument and a hypergeometric function, which depends on the scaling dimensions of the fields. General formulas for this function, $G$, are e.g. given in Wag for two and four dimensions.

We sketch now, how this form of the partial waves is reached. They are eigenfunctions of a differential operator, which one gets from the Casimir operator,

$$
\mathcal{C}=\frac{1}{2} M^{\mu \nu} M_{\mu \nu}+\frac{1}{2}\left(P_{\mu} K^{\mu}+K_{\mu} P^{\mu}\right)-D^{2},
$$

with the generators of the conformal algebra $M_{\mu \nu}, P_{\mu}, K_{\mu}$ and $D$. As this Casimir operator has the irreducible positive energy representations of the conformal group as eigenvectors and so the projections in the partial expansion are just projections onto the eigenspaces of $\mathcal{C}$, it is inserted in the four-point function,

$$
\langle\phi \phi \mathcal{C} \phi \phi\rangle,
$$

to get the partial waves. Commuting it with the fields one can pull it out of the four-point function and gets a differential operator, in which the hypergeometric differential operator is found. It acts on the function of cross ratios $s$ and $t$ in the four-point function. This is the one mentioned above, which has the partial waves as eigenfunctions.

The next step of the calculation of the partial wave expansion consists of the substitution of variables in the expression of the four-point function in terms of finitely many independent amplitudes from the section 2.2 by the chiral variables. After multiplication by $\frac{u-v}{u v}$ to compensate the factor in (2.3.3), the result can be brought into a form, which is a finite linear combination of terms,

$$
\left(u^{i} \text { or }\left(\frac{u}{1-u}\right)\right)\left(v^{j} \text { or }\left(\frac{v}{1-v}\right)^{j}\right)-\left(v^{i} \text { or }\left(\frac{v}{1-v}\right)\right)\left(u^{j} \text { or }\left(\frac{u}{1-u}\right)^{j}\right) \text {, }
$$


with $i, j \geq 0$. One still needs to expand these terms into functions $G_{n}(z)$ with the help of a few expansion rules:

$$
\begin{aligned}
z^{p} & =\sum_{n \in p+\mathbb{N}_{0}} \frac{(-1)^{n-p}(p)_{n-p}^{2}}{(n-p) !(n+p-1)_{n-p}} G_{n}(z), \\
\left(\frac{z}{1-z}\right)^{p} & =\sum_{n \in p+\mathbb{N}_{0}} \frac{(p)_{n-p}^{2}}{(n-p) !(n+p-1)_{n-p}} G_{n}(z) .
\end{aligned}
$$

Here $(i)_{j}=(i+j-1) ! /(i-1)$ ! is the rising factorial, also called Pochhammer symbol. Sorting the terms leads to

$$
\begin{aligned}
\left\langle\phi\left(x_{1}\right) \phi\left(x_{2}\right) \phi\left(x_{3}\right) \phi\left(x_{4}\right)\right\rangle= & \left\langle\phi\left(x_{1}\right) \phi\left(x_{2}\right)\right\rangle\left\langle\phi\left(x_{3}\right) \phi\left(x_{4}\right)\right\rangle \\
& \frac{u v}{u-v} \sum_{m, n} X_{m, n}\left(G_{m}(u) G_{n}(v)-G_{m}(v) G_{n}(u)\right) .
\end{aligned}
$$

Relabeling the indices the comparison with eq. (2.3.2) with eq. (2.3.3) inserted provides the partial wave coefficients

$$
B_{k, L}=\left(X_{k+L, k-1}-X_{k-1, k+L}\right) .
$$

We have seen in this section, how we get the partial wave coefficients explicitly from an expression of the four-point function, which has only finitely many monomials in conformal cross ratios $s$ and $t$. In [NRT05] the explicit results for the coefficients $B_{k, L}$ are given for four identical field with scaling dimension 4 .

However, the described steps can be performed for any such expression. We discuss in chapter 6, how the results for superconformal chiral scalar four-point functions can give such an expression for a scalar conformal four-point function contained in it. Furthermore we see, that the partial wave expansion - computed as described in this section - could be directly promoted to the whole superconformal four-point function.

\subsection{Wightman positivity}

Now we know, how to write a four-point function, as an infinite sum: the partial wave expansion. Of cause, this is much less compact than its original form, but it grants us access to the conditions, which finitely many independent amplitudes left at the end of section 2.2 have to fulfill, so that this four-point function is Wightman positive. Thus it gives a piece of the puzzle to construct a model following the Wightman axioms: Only if all Wightman functions are Wightman positive, the model lives on a Hilbert space, as such a space has a positive definite inner product. Only then its Wightman functions have a sensible physical interpretation as probabilities. But the investigation of all n-point functions is still not the sufficient condition, because also Cauchy-Schwarz inequalities resulting from the mixed terms in eq. (2.1.5) have to be considered. Actually, this has been achieved only in two dimensions so far and 
led to the proof already mentioned above, that there are only free models with fields having scaling dimension $d=2$ (cf. [NRT08]).

As mentioned in [NRT05 the certain positivity of the four-point function of massless scalar free fields leads to the conclusion, that all partial waves are positive, because all coefficients in its partial wave expansion are strictly positive.

Hence the Wightman positivity of any four-point function is equivalent to the condition

$$
B_{k, L} \geq 0
$$

In the case of four identical scaling dimensions $d=4$ (cf. [NRT05]) a compact set with non-vanishing open interior of possible five-tuples of amplitudes is left by these conditions. Higher order correlation functions are discussed in [BMRW09] and a very recent preprint [NRW11]. 


\section{Chapter 3}

\section{$\mathcal{N}=1$ Superconformal field theories}

The obvious shortcomings of the otherwise enormously successful standard model of particle physics, such as the absence of particle masses, triggered a flood of theoretical ideas. On this path beyond the standard model some theories have been developed over several decades now, but still wait for empirical proof.

At this moment many hope for the near future to solve the puzzle of mass generation by the Higgs mechanism, when Higgs particles can be found by the detectors of the Large Hadron Collider. The same experiments at this proton collider could also possibly find first particles predicted by one of the most influential developments of physics beyond the standard model: Supersymmetry.

Supersymmetric theories have not any more the strict separation of space time symmetries and internal symmetries, which is demanded, if one sticks to ordinary symmetry algebras. With the help of $\mathbb{Z}_{2}$-graded algebras called superalgebras, which use not only commutators, but also anticommutators, one can construct space time symmetries, which also relate fermions with bosons and vice versa.

The success of supersymmetry can found in its ability to potentially explain several unsolved problems at once.

First there is the hierarchy problem, which is given by the many orders of magnitudes between the different energy scales in the standard model. Pertubative calculations have to be extremely fine tuned because of this fact. While supersymmetry does not change the scales, which leave some physicist ill at ease, it provides cancellation of contributions from bosons and fermions in pertubative calculations, which give the smallness of the electro-weak scale some kind of naturalness. This can be seen as a solution to a technical issue, as pertubative calculations are only tools applied on quantum field theories.

When the supersymmetry is broken, which is needed to match our empirical knowledge of particle physics, the possible existence of heavy particles, which have not been detected yet, is another consequence of supersymmetry and could bring an explanation to hitherto unexplained experimental data. Dark matter has been indirectly proven to exist e.g. by its gravitational influence on galactic rotation curves and by graviational lensing. A supersymmetric particle is a promising candidate for 
it. Also several extremely high energetic cosmic rays have been observed, which can not be explained by the standard particle zoo. But a heavy supersymmetric particle with a low cross section with normal matter could trigger such high energy particles from space.

In this thesis the unbroken superconformal symmetry group is taken as space time symmetry group, which contains super Poincare symmetry. Already the first supersymmetric theories discussed had superconformal symmetry (cf. WZ74, DS74, HLS75]), but as the focus went to physically realistic theories most work was done on theories with super Poincare symmetry.

Still superconformal symmetry has not only toy-model qualities. Insights in the conformal invariance of supersymmetric non-Abelian gauge theories at renormalization group fixed points with vanishing $\beta$-function ([Sei95] and more recently [IW03, IW04, BIWW05]) brought new interest to a non-perturbative analysis of superconformal field theories in the mid-90s. The very recent study of new supergravity models with the potential to resolve some problems of minimal supersymmetric standard models $\mathrm{FKL}^{+} 10 \mathrm{a}, \mathrm{FKL}^{+} 10 \mathrm{~b}$. could possibly again renew attention on this topic. Here big emphasis is given to the underlying superconformal symmetry.

In this chapter we first have a look at the supersymmetry algebra and then define superspace as a coset of the group of supertranslations. In section 3.3 superconformal symmetry is discussed. The transformation properties of superspace coordinates lead to those of intervals, which we need in the next chapter to construct superconformal invariants.

In section 3.4 field representations of the supersymmetry and of superconformal symmetry are discussed. First we investigate multiplets of fields on Minkowski space and then see, how these are grouped in superfields defined on superspace.

\subsection{Super-Poincaré algebra}

Supersymmetry is generated by operators $Q_{\alpha}$ and their hermitian conjugates $Q_{\alpha}^{\dagger}=$ $\bar{Q}_{\dot{\alpha}}$. They are Weyl spinors and relate fermionic fields to bosonic fields and vice versa. They are part of a supersymmetric extension of the Poincaré algebra, the $\mathcal{N}=1$ Super-Poincaré algebra. This is a graded algebra, which can be calculated solely from the Poincaré algebra and the choice of the constant in the anti-commutator of $Q_{\alpha}$ and its conjugate,

$$
\left\{Q_{\alpha}, \bar{Q}_{\dot{\alpha}}\right\}=2 \sigma_{\alpha \dot{\alpha}}^{\mu} P_{\mu},
$$

with the translation generator, $P_{\mu}=(H,-\mathbf{P})$. This is done e.g. in QKS10 and we will here summarize it here 1

Apart from the factor the expression in eq. (3.1.1) is determined by the index structure and it is this cause, which also gives the form of two of the other missing (anti-)commutators.

The commutator of $Q$ and $P^{\mu}$ can only be proportional to $\sigma^{\mu} \bar{Q}$. With the Jacobi

\footnotetext{
${ }^{1}$ In appendix A Pauli matrices and other choices within the possibities of conventions are given.
} 
identity of $P^{\mu}, P^{\nu}$ and $Q$ it can be shown to vanish,

$$
\left[Q, P^{\mu}\right]=0 \text {. }
$$

The anti-commutator of $Q$ with itself has to get its indices from a contraction of the anti-symmetrized product of sigma-matrices , i $/ 4\left(\sigma_{\mu} \bar{\sigma}_{\nu}-\sigma_{\nu} \bar{\sigma}_{\mu}\right)$, and the generator of Lorentz transformations, $M_{\mu \nu}$. But the anti-commutator commutes with $P_{\mu}$, as we have just seen, and this contraction does not. Thus it is a consequence of Poincaré symmetry, that the generators of supersymmetry anti-commute:

$$
\{Q, Q\}=0 .
$$

From the facts, that the supersymmetry generator, $Q_{\alpha}$, is on the one hand a spinor and on the other hand transforms unitarily under Lorentz transformations with the operator $U=\exp \left(-\mathrm{i} / 2 \omega_{\mu \nu} M^{\mu \nu}\right)$, one can read off the commutator with $M^{\mu \nu}$,

$$
\left[Q, M^{\mu \nu}\right]=\frac{\mathrm{i}}{4}\left(\sigma^{\mu} \bar{\sigma}^{\nu}-\sigma^{\nu} \bar{\sigma}^{\mu}\right) Q .
$$

A symmetry with respect to tranformations of the supersymmetry generators multiplying them by a $u(1)$ phase factor, called R-symmetry, leads to the following commutation relation:

$$
[Q, R]=-\mathrm{i} \frac{1}{2} Q
$$

This commutator is frequently taken to be part of the super Poincaré symmetry. The factor is convention and corresponds here to the one we have in the superconformal algebra in app. B. Other internal symmetries commute with the Super-Poincaré algebra. The (anti-)commutators of $\bar{Q}$ are given by the conjugation of the ones of $Q$.

\subsection{Superspace}

This section defines superspace as a coset of the supertranslational group, which is the quotient of the super Poincare group and the Lorentz group. Together with the properties of the Graßmann variables this can be found in a compact form e.g. in QKK10]. The general element of the translational supergroup is given by

$$
g(z)=e^{\mathrm{i}\left(x^{\mu} P_{\mu}+\theta^{\alpha} Q_{\alpha}+\bar{Q}_{\dot{\alpha}} \bar{\theta}^{\dot{\alpha}}\right)},
$$

where we find the supersymmetry generators, $Q_{\alpha}$ and $\bar{Q}_{\dot{\alpha}}$, and the generator of translations in Minkowski space $P_{\mu}$. The parameters $z^{M}=\left(x^{\mu}, \theta^{\alpha}, \bar{\theta}^{\dot{\alpha}}\right)$ are the coordinates of superspace. $\theta^{\alpha}$ and $\bar{\theta}^{\dot{\alpha}}$ are Graßmann-valued spinors. This space we denote as $\mathbb{R}^{4 \mid 4}$ because of four real and four Graßmann coordinates.

The composition of two of these group elements can be calculated with the help of the supersymmetry algebra:

$$
\begin{aligned}
g\left(z_{1}\right) g\left(z_{2}\right)=g\left(z_{3}\right) & =e^{\mathrm{i}\left(x_{3}^{\mu} P_{\mu}+\theta_{3} Q+\bar{Q} \bar{\theta}_{3}\right)} \\
\text { with } x_{3}^{\mu} & =x_{1}^{\mu}+x_{2}^{\mu}+\mathrm{i} \theta_{1} \sigma^{\mu} \bar{\theta}_{2}-\mathrm{i} \theta_{2} \sigma^{\mu} \bar{\theta}_{1}, \\
\theta_{3}^{\alpha} & =\theta_{1}+\theta_{2}, \\
\bar{\theta}_{3}^{\alpha} & =\bar{\theta}_{1}+\bar{\theta}_{2} .
\end{aligned}
$$


With the composition of two infinitesimally separated elements of this group we get the supertranslationally invariant one form, $e^{M}=\left(e^{\mu}, \mathrm{d} \theta^{\alpha}, \mathrm{d} \bar{\theta}^{\dot{\alpha}}\right)$ :

$$
\begin{aligned}
g(z) g(z+\mathrm{d} z)=g\left(z_{3}\right) & =e^{\mathrm{i}\left(e^{\mu} P_{\mu}+\mathrm{d} \theta Q+\bar{Q} \mathrm{~d} \bar{\theta}\right)} \\
\text { with } \quad e^{\mu} & =\mathrm{d} x^{\mu}+\mathrm{i} \mathrm{d} \theta \sigma^{\mu} \bar{\theta}-\mathrm{i} \theta \sigma^{\mu} \mathrm{d} \bar{\theta} .
\end{aligned}
$$

This brings us to the invariant supersymmetric interval length and the exterior derivative on superspace :

$$
\begin{aligned}
e^{2} & =\eta_{\mu \nu} e^{\mu} e^{\nu} \\
\mathrm{d}=e^{M} D_{M} & =\left(\mathrm{d} x^{\mu}+\mathrm{id} \theta \sigma^{\mu} \bar{\theta}-\mathrm{i} \theta \sigma^{\mu} \mathrm{d} \bar{\theta}\right) \frac{\partial}{\partial x^{\mu}}+\mathrm{d} \theta^{\alpha} D_{\alpha}-\mathrm{d} \bar{\theta}^{\dot{\alpha}} \bar{D}_{\dot{\alpha}}
\end{aligned}
$$

with the covariant derivatives, $D_{M}=\left(\partial_{\mu}, D_{\alpha}, \bar{D}_{\dot{\alpha}}\right)$,

$$
\begin{aligned}
D & =\frac{\partial}{\partial \theta}-\mathrm{i} \sigma^{\mu} \bar{\theta} \frac{\partial}{\partial x^{\mu}}, \\
\bar{D} & =-\frac{\partial}{\partial \bar{\theta}}+\mathrm{i} \theta \sigma^{\mu} \frac{\partial}{\partial x^{\mu}} .
\end{aligned}
$$

The actual reason for the results in this paper to be only valid for $\mathcal{N}=1$ is the property

$$
\begin{aligned}
\theta^{\alpha} \theta^{\beta} & =-\frac{1}{2}\left(\epsilon^{-1}\right)^{\alpha \beta} \theta \tilde{\theta} \\
\bar{\theta}^{\dot{\alpha}} \bar{\theta}^{\dot{\beta}} & =\frac{1}{2}\left(\bar{\epsilon}^{-1}\right)^{\dot{\alpha} \dot{\beta}} \tilde{\bar{\theta}} \bar{\theta}
\end{aligned}
$$

with the anti-symmetric $2 \times 2$ matrices $\left(\epsilon^{-1}\right)^{\alpha \beta}$ and $\left(\bar{\epsilon}^{-1}\right)^{\dot{\alpha} \dot{\beta}}$ (cf. appendix A).

For the discussion of chiral and anti-chiral superfields it is convenient to use chiral and anti-chiral coordinates, which are respectively defined by

$$
\begin{aligned}
& x_{+}{ }^{\mu}=x^{\mu}-\mathrm{i} \theta \sigma^{\mu} \bar{\theta}, \\
& x_{-}{ }^{\mu}=x^{\mu}+\mathrm{i} \theta \sigma^{\mu} \bar{\theta} .
\end{aligned}
$$

The covariant derivatives for these coordinates are

$$
\begin{array}{ccc}
\bar{D}_{+}=-\frac{\partial}{\partial \bar{\theta}} & , & D_{+}=\frac{\partial}{\partial \theta}-2 \mathrm{i} \sigma^{\mu} \bar{\theta} \frac{\partial}{\partial x^{-\mu}}, \\
D_{-}=\frac{\partial}{\partial \theta} \quad, & \bar{D}_{-}=-\frac{\partial}{\partial \bar{\theta}}+2 \mathrm{i} \theta \sigma^{\mu} \frac{\partial}{\partial x^{+\mu}} .
\end{array}
$$

As we will see in the following chapters the correlation functions are expressed in terms of the following intervals

$$
\begin{aligned}
x_{\overline{i j}}^{\mu} & =x_{i-}{ }^{\mu}-x_{j+}{ }^{\mu}-2 \mathrm{i} \theta_{j} \sigma^{\mu} \bar{\theta}_{i}, \\
\theta_{i j}^{\alpha} & =\theta_{i}^{\alpha}-\theta_{j}^{\alpha}, \\
\bar{\theta}_{i j}^{\dot{\alpha}} & =\bar{\theta}_{i}^{\dot{\alpha}}-\bar{\theta}_{j}^{\dot{\alpha}},
\end{aligned}
$$


which transform homogeneously under superconformal transformations, as we will see in the end of section $3.3^{2}$

We define hermitian $2 \times 2$ matrices - written with straight characters - for any four-vector, $v^{\mu}$, as the contraction with Pauli matrices,

$$
\begin{aligned}
\mathrm{v}_{\alpha \dot{\alpha}} & =v^{\mu} \sigma_{\mu_{\alpha \dot{\alpha}}}, \\
\tilde{\mathrm{v}}^{\dot{\alpha} \alpha} & =v^{\mu} \bar{\sigma}_{\mu}^{\dot{\alpha} \alpha} .
\end{aligned}
$$

For the interval, $x_{i j}{ }^{\mu}$, (cf. eq. 3.2.18) ) the inverses of these matrices are

$$
\mathrm{x}_{\bar{i} j}^{-1}=\frac{\tilde{\mathrm{x}}_{\bar{i} j}}{x_{\bar{i} j}{ }^{2}}, \quad \tilde{\mathrm{x}}_{\bar{i} j}^{-1}=\frac{\mathrm{x}_{\bar{i} j}^{-}}{x_{\bar{i} j}{ }^{2}} .
$$

\subsection{Superconformal symmetry}

We define superconformal transformations, as coordinate transformations of superspace, which change the infinitesimal supersymmetric length interval in eq. 3.2.8 only by a local scale factor, $\Omega(z, g)$ :

$$
e^{2}\left(z^{\prime}\right)=\Omega^{2}(z, g) e^{2}(z) .
$$

This is satisfied by supersymmetric versions of the finite transformations known from conformal symmetry (cf. [Par98]):

- Supertranslation:

$$
\begin{aligned}
x^{\prime \mu} & =x^{\mu}+a^{\mu}+\mathrm{i} \alpha \sigma^{\mu} \bar{\theta}-\mathrm{i} \theta \sigma^{\mu} \bar{\alpha}, \\
\theta^{\prime} & =\theta+\alpha, \\
\bar{\theta}^{\prime} & =\bar{\theta}+\bar{\alpha}
\end{aligned}
$$

with $a^{M}=\left(a^{\mu}, \alpha, \bar{\alpha}\right) \in \mathbb{R}^{4 \mid 4}$.

- Superdilation:

$$
\begin{aligned}
x^{\prime \mu} & =|\tau| x^{\mu}, \\
\theta^{\prime} & =\tau^{\frac{1}{2}} \theta, \\
\bar{\theta}^{\prime} & =\bar{\tau}^{\frac{1}{2}} \bar{\theta}
\end{aligned}
$$

with $\tau^{\frac{1}{2}} \in \mathbb{C}$.

- Super-Lorentz-transformations:

$$
\begin{aligned}
x^{\prime \mu} & =\left(e^{w}\right)^{\mu}{ }_{\nu} x^{\nu}, \\
\theta^{\prime} & =\theta e^{\frac{1}{4} w^{\mu \nu} \sigma_{\mu} \bar{\sigma}_{\nu}}, \\
\bar{\theta}^{\prime} & =\bar{\theta} e^{-\frac{1}{4} w^{\mu \nu} \bar{\sigma}_{\mu} \sigma_{\nu}}
\end{aligned}
$$

with the tensor exponential defined by its series expansion and the antisymmetric tensor $w^{\mu \nu}=-w^{\nu \mu}, w \in s o(1,3)$.

\footnotetext{
${ }^{2}$ The spinoral indices will be omitted in most of the paper and a tilde is used to indicate lower indices of the spinors.
} 
- Special superconformal transformation ${ }^{3}$.

$$
\begin{aligned}
x_{+}^{\prime \mu} & =\frac{x_{+}^{\mu}-b_{-}^{\mu} x_{+}^{2}+2 \beta \sigma^{\mu} \tilde{\mathrm{x}}_{+} \tilde{\theta}}{1+x_{+}^{2} b_{-}^{2}-2 x_{+}^{\nu} b_{-\nu}-8 \beta \tilde{\beta} \theta \tilde{\theta}-4 \beta \tilde{\theta}+4 \beta \mathrm{b}_{-} \tilde{\mathrm{x}}_{+} \tilde{\theta}} \\
\theta^{\prime} & =\frac{\theta-\theta \mathrm{x}_{+} \tilde{\mathrm{b}}_{-}+4 \beta \theta \tilde{\theta}-\mathrm{i} \tilde{\bar{\beta}} \tilde{\mathrm{x}}_{+}-\mathrm{i} \tilde{\bar{\beta}} \tilde{\mathrm{b}}_{-} x_{+}^{2}+4 \mathrm{i} \beta \theta \mathrm{x}_{+} \bar{\beta}}{1+x_{+}^{2} b_{-}^{2}-2 x_{+}^{\nu} b_{-\nu}-8 \beta \tilde{\beta} \theta \tilde{\theta}-4 \beta \tilde{\theta}+4 \beta \mathrm{b}_{-} \tilde{\mathrm{x}}_{+} \tilde{\theta}}, \\
x_{-}^{\prime}{ }^{\mu} & =\frac{x_{-}^{\mu}-b_{+}^{\mu} x_{-}^{2}+2 \tilde{\bar{\theta}} \tilde{\mathrm{x}}_{+} \sigma^{\mu} \bar{\beta}}{1+x_{-}^{2} b_{+}^{2}-2 x_{-}^{\nu} b_{+\nu}-8 \tilde{\bar{\theta}} \bar{\theta} \tilde{\bar{\beta}} \bar{\beta}-4 \tilde{\bar{\theta}} \bar{\beta}+4 \tilde{\bar{\theta}} \tilde{\mathrm{x}}_{-} \mathrm{b}_{+} \bar{\beta}} \\
\bar{\theta}^{\prime} & =\frac{\bar{\theta}-\tilde{\mathrm{b}}_{+} \mathrm{x}_{-} \bar{\theta}+4 \bar{\beta} \tilde{\bar{\theta}} \bar{\theta}-\mathrm{i} \tilde{\mathrm{x}}-\tilde{\beta}-\mathrm{i} \tilde{\mathrm{b}}_{+} \tilde{\beta} x_{-}^{2}+4 \mathrm{i} \bar{\beta} \beta \mathrm{x}_{-} \bar{\theta}}{1+x_{-}^{2} b_{+}^{2}-2 x_{-}^{\nu} b_{+\nu}-8 \tilde{\bar{\theta}} \tilde{\bar{\beta}} \tilde{\bar{\beta}}-4 \tilde{\bar{\theta}} \bar{\beta}+4 \tilde{\bar{\theta}} \tilde{\mathrm{x}}_{-} \mathrm{b}_{+} \bar{\beta}}
\end{aligned}
$$

with $b^{M}=\left(b^{\mu}, \beta, \bar{\beta}\right) \in \mathbb{R}^{4 \mid 4}$. Here we used the same notation as in eqns. (3.2.14) and 3.2.15): $b_{ \pm}{ }^{\mu}=b^{\mu} \mp \mathrm{i} \beta \sigma^{\mu} \bar{\beta}$. The straight characters denote again the matrix resulting from the contraction with the Pauli matrices (cf. eqns. (3.2.21) and (3.2.22).

The special superconformal transformations are written here for chiral and antichiral coordinates, because the equations for $x_{+}$and $x_{-}$are shorter than the one for $x$. The latter, of course, is just half of the sum of the former two and so it is for their transformations.

The form of the transformations of $x_{+}$and $x_{-}$also gives a glimpse on a property, which may be taken as a defining property for continuous superconformal transformations, as it was done in BK95. The superconformal transformation of chiral coordinates are functions of chiral coordinates again and the corresponding statement is true for anti-chiral coordinates.

The superdilations can be split into a conformal contribution, the dilations, and a contribution of supersymmetry, the R-symmetry, if we define $\tau^{\frac{1}{2}}=\lambda^{\frac{1}{2}} e^{\mathrm{i} \frac{1}{2} \Omega}$ with $\lambda \in \mathbb{R}^{+}$and the phase $\Omega \in \mathbb{R}\left(e^{\mathrm{i} \Omega} \in S^{1}\right)$ :

- Dilation:

$$
\begin{aligned}
x^{\prime \mu} & =\lambda x^{\mu}, \\
\theta^{\prime} & =\lambda^{\frac{1}{2}} \theta, \\
\bar{\theta}^{\prime} & =\lambda^{\frac{1}{2}} \bar{\theta} .
\end{aligned}
$$

- R-symmetry:

$$
\begin{aligned}
x^{\prime \mu} & =x^{\mu}, \\
\theta^{\prime} & =e^{\mathrm{i} \frac{1}{2} \Omega} \theta, \\
\bar{\theta}^{\prime} & =e^{-\mathrm{i} \frac{1}{2} \Omega} \bar{\theta} .
\end{aligned}
$$

\footnotetext{
${ }^{3}$ In Par98 the expressions in the derivation of this transformation suffer from contradicting signs. The correct formulas appear in Par99.
} 
Once the generator of infinitesimal superconformal transformations is found, one is quickly led to the superconformal algebra. In [Par99] this generator is calculated and for $\mathcal{N}=1$ that result gets:

$$
\mathcal{L}=h^{\mu} \partial_{\mu}+\zeta D+\tilde{\bar{\zeta}} \tilde{\bar{D}}
$$

where $\left(h^{\mu}, \zeta, \bar{\zeta}\right)$ has the components

$$
\begin{aligned}
h^{\mu} & =\frac{1}{2} \operatorname{tr}\left(\sigma^{\mu} \tilde{\mathrm{h}}\right), \\
\zeta & =-\mathrm{i} \frac{1}{8} \bar{D} \tilde{\mathrm{h}} \\
\tilde{\bar{\zeta}} & =\mathrm{i} \frac{1}{8} \tilde{D} \mathrm{~h}
\end{aligned}
$$

with

$$
\begin{aligned}
\tilde{\mathrm{h}}= & \sigma^{\mu} \tilde{\mathrm{x}}_{+} \mathrm{b} \tilde{\mathrm{x}}_{-}-\tilde{\mathrm{x}}_{-}\left(\frac{1}{4} w_{\mu \nu} \sigma^{\mu} \bar{\sigma}^{\nu}-\frac{1}{2} \lambda-4 \theta \tilde{\beta}\right) \\
& \left(\frac{1}{4} w_{\mu \nu} \bar{\sigma}^{\mu} \sigma^{\nu}+\frac{1}{2} \lambda+4 \tilde{\bar{\beta}} \bar{\theta}\right) \tilde{\mathrm{x}}_{+}+2 \Omega \bar{\theta} \theta+4 \mathrm{i}(\bar{\alpha} \theta-\bar{\theta} \alpha)+\tilde{\mathrm{a}} .
\end{aligned}
$$

The commutator of two copies of this generator is, of course, again of this form, as the algebra has to close:

$$
\left[\mathcal{L}_{1}, \mathcal{L}_{2}\right]=\mathcal{L}_{3}
$$

This contains the information, how the coefficients for an infinitesimal transformation looks like, which results from two successive arbitrary infinitesimal superconformal transformations. In app. B we see, how this also gives us the superconformal algebra in terms of its basic generators and state the whole algebra.

Furthermore the same information is contained in the matrix commutator with $(4 \mid 1) \times(4 \mid 1)$ supermatrices, which means, that it has a block structure with $4 \times 4$ and $1 \times 1$ real components on the diagonal and the rest Graßmann components, (cf. [Par99]) of the following kind:

$$
M=\left(\begin{array}{ccc}
\frac{1}{4} w_{\mu \nu} \bar{\sigma}^{\mu} \sigma^{\nu}+\frac{1}{2} \lambda+\mathrm{i} \frac{1}{6} \Omega & -\mathrm{i} \tilde{a} & 2 \bar{\alpha} \\
-\mathrm{ib} & \frac{1}{4} w_{\mu \nu} \sigma^{\mu} \bar{\sigma}^{\nu}-\frac{1}{2} \lambda+\mathrm{i} \frac{1}{6} \Omega & 2 \tilde{\beta} \\
2 \tilde{\bar{\beta}} & 2 \alpha & \mathrm{i} \frac{2}{3} \Omega
\end{array}\right) .
$$

This is a general supertraceless supermatrix with

$$
B M B^{-1}=-M^{\dagger}
$$

and

$$
B=\left(\begin{array}{ccc}
0 & 1 & 0 \\
1 & 0 & 0 \\
0 & 0 & -1
\end{array}\right)
$$

But most important is, that these supermatrices allow us to identify the superconformal group with the $(16 \mid 8)$ dimensional group $S U(2,2 \mid 1)$ generated by these 
supermatrices, as demonstrated in [Par99]. As we have done already above, left of the vertical dash real components and on the other side Graßmann components are considered. In summary we have the following infinitesimal parameters. Two fourvectors, $a^{\mu}$ and $b^{\mu}$, two real parameters, $\lambda$ and $\Omega$, and the six Lorentz parameters, $\omega \in s o(1,3)$, are in total 16 real parameters and thus one more - the R-symmetry than the conformal transformations in four dimensions have. The Graßmann spinors $\alpha, \bar{\alpha}, \beta$ and $\bar{\beta}$ have eight components.

We continue in the line of [Par99], when we define the following element of the supermatrix representation of the superconformal group,

$$
G(g)=e^{-M(g)},
$$

with an element, $g$, of the superconformal group.

A subgroup is the one of the supertranslations, of which we have already seen a representation in eq. (3.2.1) and thus know, that its parameters form superspace by definition. The restriction of the representation in eq. 3.3.30 to this subgroup is given by parameters $\left(a^{\mu}, \alpha, \bar{\alpha}\right)$ and all other parameters are zero. If we identify these nonzero parameters again with superspace and calculate the exponential, we get the following form of elements of this representation of the supertranslational group:

$$
G_{T}(z)=\left(\begin{array}{ccc}
1_{2 \times 2} & -\tilde{\mathrm{x}}_{+} & 2 \bar{\theta} \\
0 & 1_{2 \times 2} & 0 \\
0 & 2 \theta & 1
\end{array}\right)
$$

There is a unique decomposition of any element $G(g)$ into $G_{T}\left(b^{\mu}, \beta, \bar{\beta}\right) G_{0}(g)$.

This enables us to get any point in superspace represented by a $(2 \mid 1) \times(4 \mid 1)$ supermatrix from the origin of superspace:

$$
Z(z)=G_{T}(z) Z_{0}=G_{T}(z)\left(\begin{array}{cc}
0 & 0 \\
1_{2 \times 2} & 0 \\
0 & 1
\end{array}\right)=\left(\begin{array}{cc}
-\mathrm{i} \tilde{\mathrm{x}}_{+} & 2 \bar{\theta} \\
1_{2 \times 2} & 0 \\
2 \theta & 1
\end{array}\right) .
$$

Under a superconformal transformation, $g: z \rightarrow z^{\prime}, G_{T}$ is mapped to $G_{T}\left(z^{\prime}\right)$,

$$
G_{T}\left(z^{\prime}\right)=G(g)^{-1} G_{T}(z) G_{0}(z, g),
$$

with a $G(g)$ as in eq. 3.3.30 and a suitable element $G_{0}(z, g) \in G_{0}$. This can be easily verified for an infinitesimal transformation, where

$$
\begin{aligned}
& G_{T}\left(z^{\prime}\right)=(1+M) G_{T}(z)\left(1-\hat{M}_{0}\right) \quad \Rightarrow \quad \delta G_{T}(z)=M G_{T}(z)-G_{T}(z) \hat{M}_{0} \\
& \text { with } G(g)=e^{-M}, G_{0}(z, g)=e^{-\hat{M}_{0}(z, g)} \\
& \qquad \hat{M}_{0}(z, g)=\left(\begin{array}{ccc}
\hat{w}(z)+\frac{1}{2} \hat{\lambda}(z)+\mathrm{i} \frac{1}{6} \hat{\Omega}(z) & 0 & 0 \\
-\mathrm{i} \hat{\mathrm{b}}(z) & \tilde{\hat{w}}(z)-\frac{1}{2} \hat{\lambda}(z)+\mathrm{i} \frac{1}{6} \hat{\Omega}(z) & 2 \tilde{\hat{\beta}} \\
2 \tilde{\hat{\beta}} & 0 & \mathrm{i} \frac{1}{3} \hat{\Omega}
\end{array}\right),
\end{aligned}
$$


where the parameters are functions of $z$ :

$$
\begin{aligned}
\hat{w}(z) & =\frac{1}{4} w_{\mu \nu} \bar{\sigma}^{\mu} \sigma^{\nu}+4 \tilde{\bar{\beta}} \bar{\theta}+\tilde{\mathrm{x}}_{-} \mathrm{b}-\frac{1}{2} \operatorname{tr}\left(4 \tilde{\bar{\beta}} \bar{\theta}+\tilde{\mathrm{x}}_{-} \mathrm{b}\right) \mathbb{1} \\
\tilde{\hat{w}}(z) & =-\hat{w}(z)^{\dagger} \\
\hat{\lambda}(z) & =\frac{1}{4} \partial_{\mu} h^{\mu}(z) \\
\hat{\Omega}(z) & =\Omega+6 \theta \mathrm{b} \bar{\theta}+6 \mathrm{i}(\theta \tilde{\beta}+\tilde{\bar{\beta}} \bar{\theta}), \\
\tilde{\hat{\beta}}(z) & =-\mathrm{i} \frac{1}{4} \sigma^{\mu} \partial_{\mu} \bar{\zeta} \tilde{\hat{\beta}}(z)=\tilde{\hat{\beta}}(z)^{\dagger}
\end{aligned}
$$

with $h_{\mu}$ and $\bar{\zeta}$ from eqns. 3.3.22 and (3.3.24), respectively.

Now we can write the transformation of $Z(z)$ in eq. 3.3.32, as

$$
\delta Z(z)=M Z(z)-Z(z) H(z, g),
$$

where $H(z)$ is defined as

$$
\hat{M}_{0}(z, g) Z_{0}=Z_{0} H(z, g)
$$

We also need the following conjugate of $Z(z)$ to get in the end the representation of the superconformal group, which acts on $\mathbb{R}^{4 \mid 4}$ :

$$
\bar{Z}(z)=\bar{Z}_{0} G_{Z}(z)^{-1}=\left(\begin{array}{ccc}
1_{2 \times 2} & 0 & 0 \\
0 & 0 & 1
\end{array}\right) G_{Z}(z)^{-1}=\left(\begin{array}{ccc}
1_{2 \times 2} & \mathrm{i} \tilde{\mathrm{x}}_{-} & -2 \bar{\theta} \\
0 & -2 \theta & 1
\end{array}\right) .
$$

It changes under infinitesimal superconformal transformations as

$$
\delta \bar{Z}(z)=\bar{H}(z, g) \bar{Z}(z)-\bar{Z}(z) M
$$

with $H(z)$ given by

$$
\bar{Z}_{0} \hat{M}_{0}(z, g)=\bar{H}(z, g) \bar{Z}_{0} .
$$

We now get back to finite transformations of $Z(z)$ and $\bar{Z}(z)$. We first note, that for continuous finite transformations, $z \rightarrow z^{\prime}$, we can find a path $z_{t}$ with $t \in[0,1]$ in superspace, such that $z_{0}=z$ and $z_{1}=z^{\prime}$ and infinitesimally separated points on this track are related to each other by an infinitesimal superconformal transformation. For any function of superspace this can be formulated as a differential equation using the generator of infinitesimal transformations, $\mathcal{L}$ from eq. (3.3.21):

$$
\frac{\partial}{\partial t} f\left(z_{t}\right)=\mathcal{L} f\left(z_{t}\right)
$$

If we take this function to be $Z(z)$, we get

$$
\frac{\partial}{\partial t} Z\left(z_{t}\right)=M Z\left(z_{t}\right)-Z\left(z_{t}\right) H\left(z_{t}, g\right)
$$

where we take the behaviour of the function $Z$ under infinitesimal transformations directly from equation 3.3.41. 
This is solved by

$$
Z\left(z_{t}\right)=e^{t M} Z(z) K(z, t, g)
$$

with the appropriate $K(z, t, g)$, which is the unit matrix for $t=0$ and solves

$$
\frac{\partial}{\partial t} K(z, t, g)=-K(z, t, g) H\left(z_{t}, g\right) .
$$

We are interested here in the finite transformation given by $t=1$, just write $K(z, g)=K(z, 1, g)$ and insert $G(g)$ again:

$$
Z\left(z^{\prime}\right)=G(g)^{-1} Z(z) K(z, g) .
$$

In comparison to eq. (3.3.33) we find, that the form of $K(z, g)$ is given by the part of the matrix $\hat{M}_{0}(z, g)$ in eq. 3.3.35):

$$
G_{0}(z, g) Z_{0}=e^{-M_{0}(z, g)} Z_{0}=Z_{0} K(z, g) \quad \Rightarrow \quad K(z, g)=\left(\begin{array}{cc}
L\left(z_{+}, g\right) & 2 \Sigma(z, g) \\
0 & u(z, g)
\end{array}\right) .
$$

As $L\left(z_{+}, g\right)$ results only from the component of $M_{0}$ in the middle, it can be easily shown, that it only depends on chiral coordinates, $z_{+}=\left(x_{+}, \theta\right)$. Furthermore we can, of course, split it into its determinant and an element $\hat{L}\left(z_{+}, g\right) \in S L(2, \mathbb{C})$ :

$$
\begin{aligned}
\Omega_{+}\left(z_{+}, g\right) & =\operatorname{det}\left(L\left(z_{+}, g\right)\right) \\
\hat{L}\left(z_{+}, g\right) & =\frac{L\left(z_{+}, g\right)}{\Omega_{+}\left(z_{+}, g\right)^{\frac{1}{2}}} .
\end{aligned}
$$

Because of the triangular structure of $K(z, g)$ and also of the lower right $2 \times 2$ block in $M_{0}(z, g)$, we can directly get $u(z, g)$. It is just the exponent of the negative lower right component of $M_{0}(z, g)$ :

$$
u(z, g)=e^{-\mathrm{i} \frac{1}{3} \hat{\Omega}(z, g)}=\Upsilon(z, g)^{-\frac{1}{3}} \in U(1),
$$

where we also introduced $\Upsilon(z, g)$, which is frequently used in the following.

Analogously we get the transformation of $\bar{Z}(z)$ :

$$
\bar{Z}\left(z^{\prime}\right)=\bar{K}(z, g) \bar{Z}(z) G(g)
$$

with

$$
\begin{aligned}
\bar{K}(z, g) & =\left(\begin{array}{cc}
\bar{L}\left(z_{-}, g\right) & 0 \\
2 \bar{\Sigma}(z, g) & u^{-1}(z, g)
\end{array}\right) \\
\Omega_{-}\left(z_{-}, g\right) & =\operatorname{det}\left(\bar{L}\left(z_{-}, g\right)\right)=\Omega_{+}\left(z_{+}, g\right)^{*}, \\
\hat{\bar{L}}\left(z_{-}, g\right) & =\frac{\bar{L}\left(z_{-}, g\right)}{\Omega_{-}\left(z_{-}, g\right)^{\frac{1}{2}}}=\hat{L}\left(z_{+}, g\right)^{\dagger} \in S L(2, \mathbb{C}), \\
\bar{\Sigma}(z, g) & =\Sigma(z, g)^{\dagger} .
\end{aligned}
$$

We also define for later use

$$
\Omega(z, g)=\sqrt{\Omega_{+}\left(z_{+}, g\right) \Omega_{-}\left(z_{-}, g\right)} .
$$


These matrices $K(z, g)$ and $\bar{K}(z, g)$ are the key to the construction of invariants because they give the transformation rules for the superconformal intervals, out of which the invariants are build. We see this point, if we take the product,

$$
\bar{Z}\left(z_{1}\right) Z\left(z_{2}\right)=\left(\begin{array}{cc}
\mathrm{i}_{\overline{1}} & -2 \bar{\theta}_{12} \\
-2 \theta_{12} & 1
\end{array}\right)
$$

with the intervals in eqns. (3.2.18)-(3.2.20). The superconformal transformation of this product is given by eqns. (3.3.50) and 3.3 .55$)$ :

$$
\bar{Z}\left(z_{1}^{\prime}\right) Z\left(z_{2}^{\prime}\right)=\bar{K}\left(z_{1}, g\right) \bar{Z}\left(z_{1}\right) Z\left(z_{2}\right) K\left(z_{2}, g\right) .
$$

This results in the following rules for the components of $\bar{Z}\left(z_{1}\right) Z\left(z_{2}\right)$ :

$$
\begin{aligned}
\tilde{\mathrm{x}}_{\overline{1} 2}^{\prime} & =\bar{L}\left(z_{1-}, g\right) \tilde{\mathrm{x}}_{\overline{1} 2} L\left(z_{2+}, g\right), \\
\theta_{12}^{\prime} & =-\mathrm{i} \bar{\Sigma}\left(z_{1}, g\right) \tilde{\mathrm{x}}_{\overline{1} 2} L\left(z_{2+}, g\right)+u^{-1}\left(z_{1}, g\right) \theta_{12} L\left(z_{2+}, g\right), \\
\bar{\theta}_{12}^{\prime} & =-\mathrm{i} \bar{L}\left(z_{1-}, g\right) \tilde{\mathrm{x}}_{\overline{1} 2} \Sigma\left(z_{2}, g\right)+\bar{L}\left(z_{1-}, g\right) \bar{\theta}_{12} u\left(z_{2}, g\right) .
\end{aligned}
$$

The first line is one of the essential transformation rules, which we need in the next chapter. For another one we first have to look at its inverse:

$$
\left(\tilde{\mathrm{x}}_{\overline{1} 2}^{-1}\right)^{\prime}=\left(L\left(z_{2+}, g\right)\right)^{-1} \tilde{\mathrm{x}}_{\overline{1} 2}^{-1}\left(\bar{L}\left(z_{1-}, g\right)\right)^{-1}
$$

The product of this one with eq. (3.3.64) has the remarkable property, that it transforms only with terms depending on $z_{1}$ :

$$
\left(\theta_{12} \tilde{\mathrm{x}}_{\overline{1} 2}^{-1}\right)^{\prime}=-\mathrm{i} \bar{\Sigma}\left(z_{1}, g\right) \bar{L}^{-1}\left(z_{1-}, g\right)+u^{-1}\left(z_{1}, g\right) \theta_{12} \tilde{\mathrm{x}}_{\overline{1} 2}^{-1} \bar{L}^{-1}\left(z_{1-}, g\right) .
$$

Of cause, we can find a similar expression for $\bar{\theta}_{21}$ because of their relation by complex conjugation. Here we can see this easily, if we swap the indices 1 and 2 in eqns. (3.3.66) and 3.3.65) and take the product of the results:

$$
\left(\tilde{\mathrm{x}}_{\overline{2} 1}^{-1} \bar{\theta}_{21}\right)^{\prime}=-\mathrm{i} L^{-1}\left(z_{1+}, g\right) \Sigma\left(z_{1}, g\right)+L^{-1}\left(z_{1+}, g\right) \tilde{\mathrm{x}}_{\overline{2} 1}^{-1} \bar{\theta}_{21} u\left(z_{1}, g\right) .
$$

These transformation properties lay the basis for the superconformal invariants, which we discuss in the next chapter.

\subsection{Supersymmetric and superconformal field represen- tations}

In this section we first take a look at some field representations of supersymmetry alone and then see, how we proceed from these representations to the ones of the superconformal algebra. They can be expressed as supermultiplets, which contain several component fields living on Minkowski space. We restrict the discussion of these multiplets to the scalar ones, which are also the starting point on the way to scalar superconformal multiplets. Afterwards we use Graßmann variables to arrange the component fields in a multiplet in one superfield living on superspace. 


\subsubsection{Supersymmetry multiplets}

The starting point is a complex scalar field, $\phi\left(x^{\mu}\right)$, which correspond to a representation of the Poincaré symmetry with spin 0 . One can construct a representation of supersymmetry from this field, if one applies the supersymmetry generators, $Q$ and $\bar{Q}$, to it. The results have to be spinoral and can be defined as a factor times the fields $\chi$ and $\bar{\chi}$ :

$$
\begin{aligned}
{\left[\phi, Q_{\alpha}\right] } & =\mathrm{i} \tilde{\chi}_{\alpha} \\
{\left[\phi, \bar{Q}_{\dot{\alpha}}\right] } & =\mathrm{i} \tilde{\bar{\chi}}_{\dot{\alpha}}
\end{aligned}
$$

Note that $\chi$ and $\bar{\chi}$ are looked at as independent spinor fields here. Then one can apply the supersymmetry operators to these two new spinoral fields and define new fields again. But at this stage one has restrictions from the supersymmetry algebra. For example, the anticommutator,

$$
\left\{\tilde{\chi}_{\alpha}, Q_{\beta}\right\}=\left\{\left[\phi, Q_{\alpha}\right], Q_{\beta}\right\}=2 \mathrm{i} M_{\alpha \beta},
$$

appears in the following graded Jacobi identity, which has only two term because $Q$ anticommutes with itself:

$$
\left\{\left[\phi, Q_{\alpha}\right], Q_{\beta}\right\}+\left\{\left[\phi, Q_{\beta}\right], Q_{\alpha}\right\}=0 .
$$

The consequence is, that

$$
M_{\alpha \beta}=\epsilon_{\alpha \beta} M .
$$

In this way one can continue and finds, that the multiplet has a finite number of components, as the algebra leads to a closure of the supersymmetric transformations within the component fields. Thus all (anti-)commutators of the component fields with the supersymmetry generators are finally given by these component fields or derivatives of them. For the chiral multiplet - a restriction of this general multiplet, which we discuss below - all steps are given, for example, in Soh85. The infinitesimal supersymmetric transformations can be expressed with the help of infinitesimal spinoral entities, $\eta$ and $\bar{\eta}$ :

$$
\delta f=-\mathrm{i}[f, \eta Q+\bar{Q} \bar{\eta}],
$$

where $f$ stands for any component fields. Thus we get the following infinitesimal variations under supersymmetry transformations:

$$
\begin{aligned}
\delta \phi & =\eta \tilde{\chi}+\tilde{\bar{\chi}} \bar{\eta} \\
\delta \chi & =-2 \eta M-\mathrm{i} \tilde{\bar{\eta}} \bar{\sigma}^{\mu} \partial_{\mu} \phi-\tilde{\bar{\eta}} \tilde{\mathrm{V}}, \\
\delta \bar{\chi} & =2 \bar{\eta} N+\mathrm{i} \bar{\sigma}^{\mu} \tilde{\eta} \partial_{\mu} \phi-\tilde{\mathrm{V}} \tilde{\eta} \\
\delta M & =\tilde{\bar{\lambda}} \bar{\eta}-\frac{1}{2} \mathrm{i} \partial_{\mu} \chi \sigma^{\mu} \bar{\eta} \\
\delta N & =\eta \tilde{\lambda}+\frac{1}{2} \mathrm{i} \eta \sigma^{\mu} \partial_{\mu} \bar{\chi} \\
\delta V_{\mu} & =\eta \sigma_{\mu} \bar{\lambda}+\lambda \sigma_{\mu} \bar{\eta}+\frac{1}{2} \mathrm{i} \eta \sigma_{\nu} \bar{\sigma}_{\mu} \partial^{\nu} \tilde{\chi}-\frac{1}{2} \mathrm{i} \partial^{\nu} \tilde{\bar{\chi}} \bar{\sigma}_{\mu} \sigma_{\nu} \bar{\eta}
\end{aligned}
$$




$$
\begin{aligned}
\delta \lambda & =-2 \eta D-\mathrm{i} \tilde{\bar{\eta}} \bar{\sigma}^{\mu} \partial_{\mu} N-\frac{1}{2} \mathrm{i} \eta \sigma^{\nu} \bar{\sigma}^{\mu} \partial_{\nu} V_{\mu}, \\
\delta \bar{\lambda} & =2 \bar{\eta} D+\mathrm{i} \bar{\sigma}^{\mu} \tilde{\eta} \partial_{\mu} M+\frac{1}{2} \mathrm{i} \bar{\sigma}^{\mu} \sigma^{\nu} \bar{\eta} \partial_{\nu} V_{\mu}, \\
\delta D & =\frac{1}{2} \mathrm{i} \partial_{\mu}\left(\eta \sigma^{\mu} \bar{\lambda}-\lambda \sigma^{\mu} \bar{\eta}\right) .
\end{aligned}
$$

This is the general multiplet $\left(\phi, \chi^{\alpha}, \bar{\chi}^{\dot{\alpha}}, M, N, V_{\mu}, \lambda^{\alpha}, \bar{\lambda}^{\dot{\alpha}}, D\right)$. It has four complex (pseudo-)scalar: 4 , one complex vector field and so sixteen bosonic components in total and four complex Weyl spinors, so that the fermionic components have also altogether sixteen real degrees of freedom. This equality of the number of bosonic and fermionic field components is generally fulfilled.

We get smaller multiplets by suitable restriction on this general one. One of them is just the restriction to real bosonic components and in consequence $\bar{\chi}=\chi^{\dagger}$ and $\bar{\lambda}=\lambda^{\dagger}$.

In this thesis we will concentrate on the chiral multiplet, for which we restrict the transformation properties. We demand, that

$$
\left[\phi, \bar{Q}_{\dot{\alpha}}\right]=0 \text {. }
$$

This leads to the smallest possible multiplet in four dimensions. It contains only three fields: two complex scalars and a Weyl spinor. $\phi$ has to be complex because otherwise the conjugation of eq. (3.4.16) and (3.1.1) shows, that $\phi$ is constant.

We define the Weyl spinor a bit different to fit a commonly used normalization for chiral fields:

$$
\left[\phi, Q_{\alpha}\right]=\mathrm{i} \sqrt{2} \tilde{\psi}_{\alpha}
$$

This way one splits the factor two in eq. 3.4.3 into two parts, as we evaluate the algebra and define $F$ to get

$$
\left\{\psi_{\alpha}, Q_{\beta}\right\}=\mathrm{i} \sqrt{2} \epsilon_{\alpha \beta} F .
$$

Then the infinitesimal transformations of the chiral multiplet, $(\phi, \psi, m)$, are given by

$$
\begin{aligned}
\delta \phi & =\sqrt{2} \eta \tilde{\psi} \\
\delta \psi & =-\mathrm{i} \sqrt{2} \tilde{\bar{\eta}} \bar{\sigma}^{\mu} \partial_{\mu} \phi+\sqrt{2} \eta m \\
\delta m & =-\mathrm{i} \sqrt{2} \partial_{\mu} \psi \sigma^{\mu} \bar{\eta} .
\end{aligned}
$$

Chiral supermultiplets are essential for the supersymmetric extension of the standard model. In the standard model left-handed and right-handed components of fermions have different gauge transformations. So in a minimal supersymmetric extension they have to have different spin 0 superpartner and thus be contained in different multiplets. Chiral multiplets contain only one Weyl fermion, which can be either the right- or left-handed components. Consequently all fundamental fermions

${ }^{4}$ These transformation properties are perhaps clearer in four component notation (e.g. Soh85]) 
in the Minimal Supersymmetric extension of the Standard Model (MSSM) are contained in chiral multiplets (cf. e.g. Sha03]).

Alternative to the definition of the chiral multiplet by the restriction in eq. (3.4.16) one can demand the commutator with $Q$ to vanish. This condition is also fulfilled by the complex conjugate of $\phi$. Following this thought we call the complex field in the following commutator $\phi^{*}$ :

$$
\left[\phi^{*}, Q_{\alpha}\right]=0 .
$$

This defines the antichiral multiplet, which has the following supersymmetric transformation properties:

$$
\begin{aligned}
\delta \phi^{*} & =\sqrt{2} \tilde{\bar{\psi}} \bar{\eta} \\
\delta \bar{\psi} & =\mathrm{i} \sqrt{2} \eta \sigma^{\mu} \partial_{\mu} \phi^{*}+\sqrt{2} \bar{\eta} m^{*}, \\
\delta m^{*} & =\mathrm{i} \sqrt{2} \eta \sigma^{\mu} \partial_{\mu} \bar{\psi} .
\end{aligned}
$$

\subsubsection{Superconformal multiplets}

So far we have discussed multiplets on the basis of the super-Poincaré algebra. As this is a subalgebra of the superconformal algebra, we proceed from the general supersymmetric multiplets to the general superconformal multiplets applying the additional symmetry. If we look at the superconformal algebra given in appendix $B$, we find, that the key is the action of the special superconformal generators $K_{\mu}, S$ and $\bar{S}$, where the latter two are given as the commutator of $K_{\mu}$ and the supersymmetry generators $Q$ and $\bar{Q}$.

While Wigner's little group method for ordinary conformal symmetry views momentum space (cf. Wig39), we look at the little group here at the origin of position space. The little group in the superconformal case contains the Lorentz transformations, dilations, special superconformal and R-symmetry transformations. Moreover there are further restrictions - in contrast to conformal symmetry - from the commutators of the special superconformal generator and the supersymmetry generators.

As mentioned above, the general supersymmetry multiplet in eqns. 3.4.7(3.4.15) is the starting piont. We do not compute the whole multiplet here, as this would be too lengthy and not needed, as we only discuss in the following the chiral and anti-chiral multiplets. The following steps lead to the general superconformal multiplet:

- It is evaluated at $x^{\mu}=0$ and we focus first on the action of the little group on $\phi$, as the other component fields result form the repeated application of supersymmetry transformations on this field. We have the commutators,

$$
\begin{aligned}
{[\phi(0), D] } & =\mathrm{i} \eta \phi(0), \\
{[\phi(0), R] } & =\mathrm{i} \kappa \phi(0) \\
{\left[\phi(0), M_{\mu \nu}\right] } & =\Sigma_{\mu \nu} \phi(0), \\
{\left[\phi(0), K_{\mu}\right] } & =0
\end{aligned}
$$


The last commutator states a result already here, which follows from eq. (3.4.40) below. The algebraic relations of the generators determine those of the representations $\eta, \kappa$ and $\Sigma_{\mu \nu}$ of the dilations, the R-symmetry and the Lorentz transformations, respectively. Taking the last one as irreducible, the other two have to be constants, as we expect for the scaling dimension, $\eta$, the R-charge, $\kappa$.

- The action of the little group generators on the other component fields at the origin can be calculated with the supersymmetric transformations of the supersymmetry multiplet at the origin, the superconformal algebra and Jacobi identities. This also determines their quantum numbers $\eta$ and $\kappa$ in relation to the ones of $\phi$. For the component fields $\phi, \chi, \bar{\chi}, M, N, V_{\mu}, \bar{\lambda}, \lambda$, D have the scaling dimensions $\eta(\phi)+\Delta \eta$, where $\Delta \eta$ is $0,1 / 2,1 / 2,1,1,1,3 / 2,3 / 2,2$, respectively, and the R-charges $\kappa(\phi)+\Delta \kappa$, where $\Delta \kappa$ is $0,1 / 2,-1 / 2,1,-1,0$, $1 / 2,-1 / 2,0$,respectively. As the supersymmetry generators, $Q$ and $\bar{Q}$, have R-charges $1 / 2$ and $-1 / 2$, respectively (cf. eqns. (B.0.29) and (B.0.30), the repeated application of these generators cause the R-charges of the component fields accordingly.

- On the component fields the matrix representation of $M_{\mu \nu}$ on the rest of the multiplet has to be established. This can be done with the help of the Jacobi identities with component fields $f, Q$ resp. $\bar{Q}$ and $M_{\mu \nu}$. This way one gets from the representation on $f$ to the representation of the higher component field in the (anti-)commutators $[f, Q\}$ resp. $[f, \bar{Q}\}$. The fields $\phi, M$ and $N$ have the same Lorentz representation, $\Sigma_{\mu \nu}$,

$$
\begin{aligned}
{\left[\phi(0), M_{\mu \nu}\right] } & =\Sigma_{\mu \nu} \phi(0), \\
{\left[M(0), M_{\mu \nu}\right] } & =\Sigma_{\mu \nu} M(0), \\
{\left[N(0), M_{\mu \nu}\right] } & =\Sigma_{\mu \nu} N(0) .
\end{aligned}
$$

The first two fermions turn out to transform with the following matrix representation of the Lorentz transormations:

$$
\begin{aligned}
{\left[\chi(0), M_{\mu \nu}\right]=} & =\chi(0)\left(\Sigma_{\mu \nu}+\frac{1}{4} \mathrm{i}\left(\sigma_{\mu} \bar{\sigma}_{\nu}-\sigma_{\nu} \bar{\sigma}_{\mu}\right)\right), \\
{\left[\bar{\chi}(0), M_{\mu \nu}\right] } & =\left(\Sigma_{\mu \nu}-\frac{1}{4} \mathrm{i}\left(\bar{\sigma}_{\mu} \sigma_{\nu}-\bar{\sigma}_{\nu} \sigma_{\mu}\right)\right) \bar{\chi}(0) .
\end{aligned}
$$

These are in general reducible, as they are the tensor product, $\Sigma \otimes(1 / 2,0)$ and $\Sigma \otimes(0,1 / 2)$, respectively, of the representation $\Sigma$ with some parameters $\left(j_{1}, j_{2}\right)$ and the mentioned spinoral representations of the Lorentz algebra. Here the representation of the bosonic fields above, $\Sigma_{\mu \nu}$, appears again, which depends on the specific multiplet and is implicitly multiplied in this equation by a unit matrix. The calculations, which lead to these commutators use some handy identities given in the appendix in eqns. A.1.13 A.1.17.

For the higher component fields the calculation of the Jacobi identities involves also derivatives of component fields. We give an example for their Lorentz 
transformation calculated with the help of the commutator $\left[M_{\mu \nu}, P_{\rho}\right]$ (cf. eq. (B.0.4)):

$$
\left[\partial_{\rho} \phi, M_{\mu \nu}\right]=\Sigma_{\mu \nu} \partial_{\rho}-\eta_{\mu \rho} \partial_{\nu} \phi-\eta_{\nu \rho} \partial_{\mu} \phi .
$$

As mentioned already we skip the rest, which we will not need for the further discussion.

- The special superconformal generator, $K_{\mu}$, is the last generator of the little group of the point $x^{\mu}=0$, for which we have to figure out its representation on the component field. At this point we can already show, which fields may appear in the commutators. The key is its commutator with the dilation generator, $D$,

$$
\left[K_{\mu}, D\right]=-\mathrm{i} K_{\mu}
$$

The Jacobi identity of $K_{\mu}, D$ and an arbitrary component field at the origin, $f(0)$, leads to a condition on the scaling dimension, $\eta_{\kappa_{\mu}(f)}$, of the representation, $\kappa_{\mu}(f)$, of $K_{\mu}$ on the field, $f$,

$$
\begin{aligned}
0 & =\left[\left[K_{\mu}, D\right], f(0)\right]+\left[[D, f(0)], K_{\mu}\right]+\left[\left[f(0), K_{\mu}\right], D\right] \\
& =-\mathrm{i}\left[K_{\mu}, f(0)\right]+\mathrm{i} \eta_{f}\left[f(0), K_{\mu}\right]-\left[\kappa_{\mu}(f), D\right] \\
& =\kappa_{\mu}(f)\left(1-\eta_{f}+\eta_{\kappa_{\mu}(f)}\right) \\
\Rightarrow \quad \eta_{\kappa_{\mu}(f)} & =\eta_{f}-1
\end{aligned}
$$

$\eta_{f}$ is the scaling dimension of the field, $f$. The linearity of the transformation only allows terms with component fields and their derivatives. The derivatives lead to higher scaling dimensions. Thus there is no term, which can hold this condition for the lower component fields, $\phi, \chi$ and $\bar{\chi}$. Also $M$ and $N$ must have a trivial representation of $K_{\mu}$ : Only $\phi$ has the right scaling dimension, but cannot be put into a linear term, which is a four-vector.

- The (anti-)commutators of the component fields and the spinoral generators of the special superconformal algebra, $S$ and $\bar{S}$, are necessary for the next step, when we investigate the non-trivial commutators with $K_{\mu}$. We state some of them here. We use the vanishing commutators of $K_{\mu}$, which we know from the last step, in the Jacobi identity of $\phi, K_{\mu}$ and $Q$ resp. $\bar{Q}$. One summand contains the commutator of $K_{\mu}$ and $Q$ resp. $\bar{Q}$, which is essentially $\bar{S}$ resp. $S$ (cf. (B.0.24) ). The other two summands vanish, so that

$$
[\phi(0), \bar{S}]=[\phi(0), S]=0 .
$$

From this we can climb the ladder with $Q$ and $\bar{Q}$ again to get the results for the other components. Once more we look at the results with the fields evaluated at the origin, which are a bit shorter and can be translated to other coordinate points giving back the terms, which we save here. From suitable 
Jacobi identities of a component field, $f, Q$ or $\bar{Q}$ and $S$ or $\bar{S}$ it follows, that

$$
\begin{aligned}
& \{\bar{\chi}(0), S\}=\{\chi(0), \bar{S}\}=[M(0), \bar{S}]=[N(0), S]=0 \\
& \{\chi(0), S\}=-2 \Gamma_{-} \phi(0), \quad\{\bar{\chi}(0), \bar{S}\}=2 \bar{\Gamma}_{+} \phi(0), \\
& {[M(0), S]=\left(\Gamma_{-}-2 \mathrm{i}\right) \tilde{\chi}(0), \quad[N(0), \bar{S}]=-\tilde{\bar{\chi}}(0)\left(\bar{\Gamma}_{+}-2 \mathrm{i}\right)}
\end{aligned}
$$

where

$$
\begin{aligned}
& \Gamma_{ \pm}=\mathrm{i} \eta_{\phi}-\frac{1}{4}\left(\sigma^{\mu} \bar{\sigma}^{\nu}-\sigma^{\nu} \bar{\sigma}^{\mu}\right) \Sigma_{\mu \nu} \pm 3 \mathrm{i} \kappa_{\phi}, \\
& \bar{\Gamma}_{ \pm}=\mathrm{i} \eta_{\phi}-\frac{1}{4}\left(\bar{\sigma}^{\mu} \sigma^{\nu}-\bar{\sigma}^{\nu} \sigma^{\mu}\right) \Sigma_{\mu \nu} \pm 3 \mathrm{i} \kappa_{\phi} .
\end{aligned}
$$

For the other component fields we again need to consider the (anti-)commutators with the derivatives of component fields. Jacobi identities of $S$ resp. $\bar{S}$, $P_{\mu}$ and the component field lead to the results:

$$
\begin{aligned}
{\left[\partial_{\mu} \phi(0), S\right] } & =-\sigma_{\mu} \chi \overline{(0)} \\
{\left[\partial_{\mu} \phi(0), \bar{S}\right] } & =\chi(0) \sigma_{\mu} \\
\left\{\partial_{\mu} \chi, S\right\} & =-2 \mathrm{i} \Gamma-\partial_{\mu} \phi(0)-\sigma_{\mu} \bar{\sigma}^{\nu}\left(\mathrm{i} V_{\nu}(0)-\partial_{\nu} \phi(0)\right) \\
\left\{\partial_{\mu} \bar{\chi}(0), S\right\} & =2 \mathrm{i} \sigma_{\mu} \bar{\epsilon} N(0) \\
\left\{\partial_{\mu} \chi(0), \bar{S}\right\} & =2 \mathrm{i} \epsilon \sigma_{\mu} M(0) \\
\left\{\partial_{\mu} \bar{\chi}(0), \bar{S}\right\} & =2 \mathrm{i} \bar{\Gamma}_{+} \partial_{\mu} \phi(0)-\left(\mathrm{i} V_{\nu(0)}+\partial_{\nu} \phi(0)\right) \bar{\sigma}^{\nu} \sigma_{\mu}
\end{aligned}
$$

- At last we return to the special conformal generator, $K_{\mu}$. $V_{\rho}$ is the lowest component field, for which the commutator with this generator does not vanish. It can now be easily calculated from the first part of eq. 3.4 .43 , because we have $\left[\bar{Q}, K_{\mu}\right]=-\tilde{S} \sigma_{\mu}$. Furthermore we need the vanishing commutators of $K_{\mu}$ and $\chi$ and the non-vanishing commutator of $K_{\mu}$ and $\partial_{\nu} \phi$ :

$$
\left[\partial_{\nu} \phi(0), K_{\mu}\right]=-2 \Sigma_{\mu \nu} \phi(0)-2 \mathrm{i} \eta \eta_{\mu \nu} \phi(0),
$$

where $\eta$ is as above the scaling dimension of $\phi$. With this we get

$$
\left[V_{\nu}(0), K_{\mu}\right]=\left(2 \mathrm{i} \Sigma_{\mu \nu}-2 \eta \eta_{\mu \nu}-\mathrm{i} \operatorname{tr}\left(\Gamma_{-} \sigma_{\mu} \bar{\sigma}_{\nu}\right)\right) \phi(0) .
$$

One can already see from the fields appearing in all these commutators and the knowledge of the commutators of component fields and $Q$ or $\bar{Q}$, which we find implicitly in eq. 3.4.7) et sqq., that this superconformal multiplet will close.

Here we want to look at the chiral and anti-chiral multiplets, which are defined by the restrictions

$$
[\bar{Q}, \phi]=0 \quad \text { resp. } \quad\left[Q, \phi^{*}\right]=0 .
$$

This also leads to a relation between the scaling dimension and the R-charge for these representations. For the chiral field we follow this from the vanishing commutator,

$$
\begin{aligned}
0 & =[\{\bar{Q}, \tilde{\bar{S}}\}, \phi(0)] \\
& =-2 \mathrm{i}[D, \phi(0)]-\mathrm{i}\left(\bar{\sigma}^{\mu} \sigma^{\nu}-\bar{\sigma}^{\nu} \sigma^{\mu}\right)\left[M_{\mu \nu}, \phi\right]-6 \mathrm{i}[R, \phi(0)],
\end{aligned}
$$


with the help of eq. (B.0.27) and implicit unit matrices again. As this is zero for all values of the indices, the second summand has to be zero separately from the first and the third one. This restricts the possible Lorentz representations of $\phi$. The first and third summand give the relations

$$
\eta_{c h}=-3 \kappa_{c h} .
$$

Analogous to this, but with $\left[\{Q, \tilde{S}\}, \phi^{*}\right]$ we get the relation for anti-chiral multiplets,

$$
\eta_{a-c h}=3 \kappa_{a-c h} .
$$

The values for scaling dimension and R-charge of a multiplet are defined here as those of the lowest component field in it. We can finally give the superconformal transformations of the chiral multiplet analogously to the way we already gave the supersymmetry transformations of the supersymmetric general multiplet in eq. (3.4.7) et sqq.. Only three component fields are left and we also split the factor 2 from the anti-commutator $\{Q, \bar{Q}\}$ in two parts, as we have done for the chiral supersymmetric multiplet above. For the chiral multiplet the commutators with $K_{\mu}$ all vanish. So we have all transformations, which lead from one component field to another one grouped in the following commutators, with which define

$$
\delta_{s c} f(x)=-\mathrm{i}[f(x), \eta Q+\bar{Q} \bar{\eta}+\zeta S+\bar{S} \bar{\zeta}] .
$$

Here we want the commutators of the fields at any point of Minkowski space and not only the origin. The translation of the fields, $f$, is given by

$$
e^{\mathrm{i} x_{\mu} P^{\mu}} f(0) e^{-\mathrm{i} x_{\mu} P^{\mu}}=f(x) .
$$

For the translation of a (anti-)commutator both operators in it are translated and this way we get from all the (anti-)commutators with fields at the origin, which we have calculated in this section, to the (anti-)commutators of the fields at $x$ :

$$
\begin{aligned}
e^{\mathrm{i} x_{\mu} P^{\mu}}[f(0), O\} e^{-\mathrm{i} x_{\mu} P^{\mu}} & =\left[f(x), e^{\mathrm{i} x_{\mu} P^{\mu}} O e^{-\mathrm{i} x_{\mu} P^{\mu}}\right\} \\
& =\left[f(x), \sum_{i=1}^{\infty} \frac{\mathrm{i}^{i}}{i !} x^{\mu_{1}} \ldots x^{\mu_{i}}\left[P_{\mu_{1}},\left[\ldots,\left[P_{\mu_{i}}, O\right]\right]\right]\right\} .
\end{aligned}
$$

But this is not much to do for eq. (3.4.61), as the commutator of the translation generator and $S$ resp. $\bar{S}$ is just $\sigma_{\mu} \overline{\bar{Q}}$ resp. $-\tilde{Q} \sigma_{\mu}$. Here the sequence already terminates because the commutator of the latter two with $P_{\mu}$ is zero. Thus we have

$$
\begin{aligned}
e^{\mathrm{i} x_{\mu} P^{\mu}}[f(0), \zeta S] e^{-\mathrm{i} x_{\mu} P^{\mu}} & =[f(x), \zeta S]+\left[f(x), \zeta \sigma_{\mu} \tilde{\bar{Q}}\right] . \\
e^{\mathrm{i} x_{\mu} P^{\mu}}[f(0), \bar{S} \bar{\zeta}] e^{-\mathrm{i} x_{\mu} P^{\mu}} & =[f(x), \bar{S} \bar{\zeta}]-\left[f(x), \tilde{Q} \sigma_{\mu} \bar{\zeta}\right],
\end{aligned}
$$

We now plug this into eq. 3.4.61) and get

$$
\delta_{s c} f(x)=-\mathrm{i}\left([f(x), \xi Q+\bar{Q} \bar{\xi}]+e^{\mathrm{i} x_{\mu} P^{\mu}}[f(0), \zeta S+\bar{S} \bar{\zeta}] e^{-\mathrm{i} x_{\mu} P^{\mu}}\right),
$$


where we defined

$$
\xi=\eta-\tilde{\bar{\zeta}} \bar{\sigma}_{\mu} \quad, \quad \bar{\xi}=\bar{\eta}+\bar{\sigma}_{\mu} \tilde{\zeta}
$$

In the end we find

$$
\begin{aligned}
\delta_{s c} \phi & =\sqrt{2} \xi \tilde{\psi} \\
\delta_{s c} \psi & =-\mathrm{i} \sqrt{2} \tilde{\bar{\xi}} \bar{\sigma}^{\mu} \partial_{\mu} \phi+\sqrt{2} \xi m-\sqrt{2} \zeta \Gamma_{-} \phi, \\
\delta_{s c} m & =-\mathrm{i} \sqrt{2} \partial_{\mu} \psi \sigma^{\mu} \bar{\xi}-\sqrt{2} \zeta\left(\Gamma_{-}-2 \mathrm{i}\right) \tilde{\psi},
\end{aligned}
$$

with $\Gamma_{-}$given by eq. (3.4.46) and the mentioned different definitions for the chiral fields in contrast to the general multiplet, $\sqrt{2} \psi=\chi$ and $m=\sqrt{2} M$.

Analogously we get to the anti-chiral multiplet writing $\phi^{*}$ instead of $\phi$ to distinguish the lowest component field of chiral and anti-chiral multiplet and with $\sqrt{2} \bar{\psi}=\bar{\chi}$ and $m^{*}=\sqrt{2} N$ :

$$
\begin{aligned}
\delta_{s c} \phi^{*} & =\sqrt{2} \tilde{\bar{\psi}} \bar{\xi} \\
\delta_{s c} \bar{\psi} & =\mathrm{i} \sqrt{2} \xi \bar{\sigma}^{\mu} \partial_{\mu} \phi+\sqrt{2} \bar{\xi} m^{*}+\sqrt{2} \bar{\Gamma}_{+} \bar{\zeta} \phi \\
\delta_{s c} m^{*} & =\mathrm{i} \sqrt{2} \xi \sigma^{\mu} \partial_{\mu} \bar{\psi}-\sqrt{2} \tilde{\bar{\psi}} a\left(\bar{\Gamma}_{+}-2 \mathrm{i}\right) \bar{\zeta}
\end{aligned}
$$

with $\bar{\Gamma}_{+}$given by eq. (3.4.47).

\subsubsection{Superfields}

We have found supersymmetric and superconformal field representations in the last section. Given as multiplets they contain several fields, for which the transformation rules have to be given. We see in this section, that they can be given in a more handy form on superspace. These representations on superspace are called superfields and can be given just by their indices, quantum numbers and the constraints, which define them.

The general superfield is given by its expansion with respect to the Graßmann variables, where the component fields of the general multiplet (cf. eqns. (3.4.7)(3.4.7)) appear as coefficient fields:

$$
\begin{aligned}
F\left(x^{\mu}, \theta, \bar{\theta}\right)= & \phi\left(x^{\mu}\right)+\theta \tilde{\chi}\left(x^{\mu}\right)+\tilde{\bar{\theta}} \bar{\chi}\left(x^{\mu}\right)+\theta \tilde{\theta} M\left(x^{\mu}\right)+\tilde{\bar{\theta}} \bar{\theta} N\left(x^{\mu}\right)+\left(\theta \sigma_{\mu} \bar{\theta}\right) V^{\mu} \\
& +\theta \tilde{\theta} \tilde{\bar{\theta}} \bar{\lambda}\left(x^{\mu}\right)+\tilde{\bar{\theta}} \bar{\theta} \theta \tilde{\lambda}\left(x^{\mu}\right)+\theta \tilde{\theta} \overline{\bar{\theta}} \bar{\theta} D\left(x^{\mu}\right)
\end{aligned}
$$

Below we also discuss a representation of the generators of supersymmetry transformations on superspace, which gives back the transformation rules for the component fields, when it is applied to this expansion. The supersymmetric case and superconformal case differs in the respective representations of symmetry transformations on superspace acting on this superfield.

This general superfield can now be restricted by conditions, which lead to simpler superfields. The scalar chiral superfields, $\Phi$, are defined by

$$
\bar{D}_{+} \Phi\left(x_{+}^{\mu}, \theta, \bar{\theta}\right)=0
$$


and the scalar anti-chiral superfields, $\bar{\Phi}$, by

$$
D_{-} \bar{\Phi}\left(x_{-}^{\mu}, \theta, \bar{\theta}\right)=0 .
$$

Here chiral and anti-chiral coordinates and the corresponding covariant derivatives (eqns. (3.2.16) and (3.2.17) ) are already used, which is very convenient, as these conditions then simply mean, that chiral and anti-chiral fields do not depend on $\bar{\theta}$ and $\theta$, respectively.

The constraints have the corresponding consequences on the expansion of the superfields in Graßmann variables, $\theta$ and $\bar{\theta}$. As superfields are operator-valued distributions on superspace, the coefficients of this expansion are operator-valued distributions on Minkowski space - the coefficient fields - and form altogether a representations on Minkowski space. These are the multiplets, which we discussed in the last section, and we show this on the example of scalar chiral and anti-chiral superfields in this section. Because of eqns. (3.4.75) and (3.4.76) their expansions in $\theta$ and $\bar{\theta}$ using chiral resp. anti-chiral coordinates are

$$
\begin{aligned}
& \Phi\left(x_{+}^{\mu}, \theta\right)=\phi\left(x_{+}^{\mu}\right)+\sqrt{2} \theta \tilde{\psi}\left(x_{+}^{\mu}\right)+\theta \tilde{\theta} m\left(x_{+}^{\mu}\right), \\
& \bar{\Phi}\left(x_{-}^{\mu}, \bar{\theta}\right)=\phi^{*}\left(x_{-}^{\mu}\right)-\sqrt{2} \tilde{\bar{\theta}} \bar{\psi}\left(x_{-}^{\mu}\right)-\tilde{\bar{\theta}} \bar{\theta} m^{*}\left(x_{-}^{\mu}\right) .
\end{aligned}
$$

If we change coordinates to Minkowski space, the expansion gets longer, as the coefficient fields, $f$, are each expanded again,

$$
\begin{aligned}
f\left(x_{+}^{\mu}\right) & =f\left(x^{\mu}\right)-\mathrm{i} \theta \sigma_{\nu} \bar{\theta} \partial^{\nu} f\left(x^{\mu}\right)-\frac{1}{4} \theta \tilde{\theta} \tilde{\bar{\theta}} \bar{\theta} \partial_{\nu} \partial^{\nu} f\left(x^{\mu}\right), \\
f\left(x_{-}^{\mu}\right) & =f\left(x^{\mu}\right)+\mathrm{i} \theta \sigma_{\nu} \bar{\theta} \partial^{\nu} f\left(x^{\mu}\right)-\frac{1}{4} \theta \tilde{\theta} \tilde{\bar{\theta}} \bar{\theta} \partial_{\nu} \partial^{\nu} f\left(x^{\mu}\right) .
\end{aligned}
$$

And so we have

$$
\begin{aligned}
\Phi\left(x^{\mu}, \theta, \bar{\theta}\right)= & \phi\left(x^{\mu}\right)+\sqrt{2} \theta \tilde{\psi}\left(x^{\mu}\right)+\theta \tilde{\theta} m\left(x^{\mu}\right)-\mathrm{i} \theta \sigma_{\nu} \bar{\theta} \partial^{\nu} \phi\left(x^{\mu}\right) \\
& +\frac{\mathrm{i}}{\sqrt{2}} \theta \tilde{\theta} \partial_{\nu} \psi\left(x^{\mu}\right) \sigma^{\nu} \bar{\theta}-\frac{1}{4} \theta \tilde{\theta} \tilde{\bar{\theta}} \bar{\theta} \partial_{\nu} \partial^{\nu} \phi\left(x^{\mu}\right) \\
\bar{\Phi}\left(x^{\mu}, \theta, \bar{\theta}\right)= & \phi^{*}\left(x_{-}^{\mu}\right)-\sqrt{2} \tilde{\bar{\theta}} \bar{\psi}\left(x_{-}^{\mu}\right)-\tilde{\bar{\theta}} \bar{\theta} m^{*}\left(x_{-}^{\mu}\right)+\mathrm{i} \theta \sigma_{\nu} \bar{\theta} \partial^{\nu} \phi^{*}\left(x^{\mu}\right)(3.4 \\
& +\frac{\mathrm{i}}{\sqrt{2}} \tilde{\bar{\theta}} \bar{\theta} \theta \sigma^{\nu} \partial_{\nu} \bar{\psi}\left(x^{\mu}\right)-\frac{1}{4} \theta \tilde{\theta} \tilde{\bar{\theta}} \bar{\theta} \partial_{\nu} \partial^{\nu} \phi\left(x^{\mu}\right)
\end{aligned}
$$

We can represent the supersymmetry algebra and the superconformal algebra as differential operators on functions on superspace. For the supersymmetry algebra 
these operators acting on $F\left(x^{\mu}, \theta, \bar{\theta}\right)$ are defined by

$$
\begin{aligned}
{\left[F, P_{\mu}\right]=} & \mathcal{P}_{\mu} F=-\mathrm{i} \partial_{\mu} F \\
{[F, Q]=} & \mathcal{Q} F=\left(\mathrm{i} \frac{\partial}{\partial \theta}-\sigma_{\mu} \bar{\theta} \partial^{\mu}\right) F ; \\
{[F, \bar{Q}]=} & \overline{\mathcal{Q}} F=\left(\mathrm{i} \frac{\partial}{\partial \bar{\theta}}-\theta \sigma_{\mu} \partial^{\mu}\right) F \\
{\left[F, M_{\mu \nu}\right]=} & \mathcal{M}_{\mu \nu} F=-\mathrm{i}\left(x_{\mu} \partial_{\nu}-x_{\nu} \partial_{\mu}+\frac{1}{4} \theta\left(\sigma_{\mu} \bar{\sigma}_{\nu}-\sigma_{\mu} \bar{\sigma}_{\nu}\right) \frac{\partial}{\partial \theta}\right. \\
& \left.-\frac{1}{4}\left[\left(\bar{\sigma}_{\mu} \sigma_{\nu}-\bar{\sigma}_{\mu} \sigma_{\nu}\right) \bar{\theta}\right]_{\dot{\alpha}} \frac{\partial}{\partial \bar{\theta}^{\dot{\alpha}}}\right) F
\end{aligned}
$$

The infinitesimal supersymmetry transformations of the chiral and the anti-chiral multiplets, which we have seen in eqns. 3.4.19) 3.4.21 and (3.4.23)-3.4.25), can be extracted from the superfields with the operator, $-\mathrm{i}(\eta \mathcal{Q}-\overline{\mathcal{Q}} \bar{\eta})$ :

$$
\begin{aligned}
& \delta \Phi\left(x_{\mu}, \theta, \bar{\theta}\right)=-\mathrm{i}(\eta \mathcal{Q}-\overline{\mathcal{Q}} \bar{\eta}) \Phi\left(x_{\mu}, \theta, \bar{\theta}\right) \\
& \delta \bar{\Phi}\left(x_{\mu}, \theta, \bar{\theta}\right)=-\mathrm{i}(\eta \mathcal{Q}-\overline{\mathcal{Q}} \bar{\eta}) \bar{\Phi}\left(x_{\mu}, \theta, \bar{\theta}\right) .
\end{aligned}
$$

For the superconformal transformations of the superfields, we get back to the discussion of superconformal transformations in section 3.3 . Following the discussion of [Par99] it is enough to choose for a quasi-primary superfield, $F\left(x^{\mu}, \theta, \bar{\theta}\right)$, which transforms as

$$
\left(x^{\mu}, \theta, \bar{\theta}\right) \stackrel{g}{\rightarrow}\left(x^{\mu \prime}, \theta^{\prime}, \bar{\theta}^{\prime}\right) \quad F^{\prime}\left(x^{\mu^{\prime}}, \theta^{\prime}, \bar{\theta}^{\prime}\right)=F\left(x^{\mu}, \theta, \bar{\theta}\right) D\left(x^{\mu}, \theta, \bar{\theta} ; g\right),
$$

a representation, $D\left(x^{\mu}, \theta, \bar{\theta} ; g\right)$, of the subgroup of the stability group at $z=0$, $S L(2, \mathbb{C}) \times U(1) \times D$. This subgroup is generated by the elements $\hat{L}\left(z_{+}, g\right)$ from eq. (3.3.53), $\Upsilon(z, g)$ from eq. (3.3.54) and $\Omega(z, g)$ from eq. (3.3.60) of the respective factors. These go back to Lorentz transformation, dilation and R-symmetry, but include in their definition all parameters of superconformal transformations because of the integration.

For scalar superfields the $S L(2, \mathbb{C})$-representation is trivial, as it has no (spinoral) indices. The rest is in this case

$$
D\left(x^{\mu}, \theta, \bar{\theta} ; g\right)=\Omega(z, g)^{-\eta} \Upsilon(z, g)^{-\kappa} .
$$

In the case of chiral and anti-chiral superfields one finds, that the relation between $\eta$ and $\kappa$ leads to the following transformation rules,

$$
\begin{aligned}
& \Phi^{\prime}\left(x_{+}^{\mu \prime}, \theta^{\prime}\right)=\Omega_{+}\left(z_{+}, g\right)^{-\eta} \Phi\left(x_{+}^{\mu}, \theta\right) \\
& \bar{\Phi}^{\prime}\left(x_{-}^{\mu \prime}, \bar{\theta}^{\prime}\right)=\Omega_{-}\left(z_{-}, g\right)^{-\eta} \bar{\Phi}\left(x_{-}^{\mu}, \bar{\theta}\right),
\end{aligned}
$$

because

$$
\Omega_{ \pm}\left(z_{ \pm}, g\right)=\Omega(z, g) \Upsilon(z, g)^{\mp \frac{1}{3}}
$$


The infinitesimal transformations of these superfields are given by

$$
\begin{aligned}
& \tilde{\delta} \Phi\left(x_{+}^{\mu}, \theta\right)=-\left(\mathcal{L}+\eta \hat{\lambda}(z)-\frac{\mathrm{i}}{3} \eta \hat{\Omega}(z)\right) \Phi\left(x_{+}^{\mu}, \theta\right), \\
& \tilde{\delta} \bar{\Phi}\left(x_{-}^{\mu}, \bar{\theta}\right)=-\left(\mathcal{L}+\eta \hat{\lambda}(z)+\frac{\mathrm{i}}{3} \eta \hat{\Omega}(z)\right) \bar{\Phi}\left(x_{-}^{\mu}, \bar{\theta}\right),
\end{aligned}
$$

where we have $\mathcal{L}$ from eq. 3.3.21. The sums of the functions of $z$, which appear in these two expressions, depend only on $z_{+}$and $z_{-}$, respectively.

If the functions are inserted and all parameters of the superconformal transformations except $\epsilon, \bar{\epsilon}, \beta$ and $\bar{\beta}$ are set to zero, one can compare this with the results from eqns. 3.4.68) 3.4 .70 and 3.4.71) 3.4 .73 , which only contain the transformations, which relate different component fields of the multiplets. The two versions differ by a factor of $i$, which is convention, and in the version in this section the lowest component fields $\phi$ and $\phi^{*}$ are scalar fields. 


\section{Chapter 4}

\section{Invariants}

Correlation functions in conformal field theories are the product of two factors. One factor is a conformally invariant function, which includes all their degrees of freedom, i.e. all model specific content. The other one establishes the correct transformation properties of the contained fields. Thus the knowledge about conformal invariants $n$ points lays the basis for all further considerations on $n$-point conformal correlation functions.

For superconformal field theory the superspace formalism (sections 3.2 and 3.4 .3 ) allows to merge all $n$-point correlation functions of component fields of a supermultiplet into one n-point function of the corresponding superfields. While the former correlation functions depend on normal space-time, the latter are functions of $n$ points of superspace. Analogous to the conformal case the degrees of freedom of these superconformal correlation functions are contained in a function of superconformal invariants.

Therefore we construct all superconformal invariants of three and four points of superspace in this chapter and discuss their properties. While there is no two point invariant just as for conformal symmetry on Minkowski space, the three point invariants are novelties concerning this comparison.

In this analysis invariant functions are grouped into fully invariant ones (or full invariants), which are invariant under the full superconformal group, which we discussed in section 3.3, and partially invariant ones (or partial invariants) without the R-symmetry, as superconformal symmetry may also be defined without this part. The focus lies on the full invariants, as in chapter 5 we discuss almost exclusively correlation function with vanishing R-charge, which depend only on them.

All possible fully invariant functions of three points are functions of just one invariant, which is nilpotent and is our choice in section 4.1. There are also two partial invariants of three points.

In sections 4.2 and 4.3 the discussion of four-point invariants is much more intricate. There are non-nilpotent and nilpotent invariants. Every partial invariants, which is not R-symmetric and thus not a full invariant, is nilpotent.

Among the full invariants we choose a set, with which all fully invariant functions of four points of superspace can be expressed. This set contains two non-nilpotent and eight nilpotent invariants. We illuminate some properties of the invariants in 
this set, which are crucial for the further discussion. All possible monomials of the nilpotent invariants are constructed and a subset of them is chosen to provide a unique expansion of arbitrary fully invariant functions with respect to nilpotent invariants. The coefficients of this expansion are functions of the two selected nonnilpotent invariants.

We also give some expansions of invariants, which we encounter, but are not in the chosen set. Furthermore we go on to prepare for the next chapter introducing set of four nilpotent invariants, which appear in the form of the four-point function, which forms theorem 5.1. Partial invariants of four-points can be found in appendix C.4.

\subsection{Three-point invariants}

The reason for the existence of superconformal three point invariants is the interval in (3.2.18), which is not symmetric under exchange of its indices. Therefore there are six different intervals $x_{\bar{i} j}$ for $i \neq j \in\{1,2,3\}$.

They transform homogeneously according to eq. (3.3.63). If we now define the following two functions of three points, given once with contravariant and once with covariant indices,

$$
\begin{aligned}
& \tilde{\mathrm{X}}_{1+}=-\mathrm{x}_{\overline{1} 3}{ }^{-1} \mathrm{x}_{\overline{2} 3} \mathrm{x}_{\overline{2} 1}{ }^{-1}, \quad \mathrm{X}_{1+}=-\tilde{\mathrm{x}}_{\overline{2} 1}^{-1} \tilde{\mathrm{x}}_{\overline{2} 3} \tilde{\mathrm{x}}_{\overline{1} 3}^{-1}, \\
& \tilde{\mathrm{X}}_{1-}=\mathrm{x}_{\overline{1} 2}{ }^{-1} \mathrm{x}_{\overline{3} 2} \mathrm{x}_{\overline{3} 1}{ }^{-1}, \quad \mathrm{X}_{1-}=\tilde{\mathrm{x}}_{\overline{3} 1}^{-1} \tilde{\mathrm{x}}_{\overline{3} 2} \tilde{\mathrm{x}}_{\overline{1} 2}^{-1},
\end{aligned}
$$

they both have the same properties under superconformal transformations:

$$
\begin{aligned}
\mathrm{X}_{1 \pm}^{\prime} & =\left(L\left(z_{1+}, g\right)\right)^{-1} \mathrm{X}_{1 \pm}\left(\bar{L}\left(z_{1-}, g\right)\right)^{-1} \\
& =\Omega\left(z_{1}, g\right)^{-1}\left(\hat{L}\left(z_{1+}, g\right)\right)^{-1} \mathrm{X}_{1 \pm}\left(\hat{\bar{L}}\left(z_{1-}, g\right)\right)^{-1}
\end{aligned}
$$

For the second part we used equations (3.3.53), (3.3.58) and (3.3.60).

With the squares of the four-vectors corresponding to $\tilde{\mathrm{X}}_{1+}$ and $\mathrm{X}_{1-}$

$$
X_{1+}^{2}=\frac{x_{\overline{2} 3}^{2}}{x_{\overline{2} 1}^{2} x_{\overline{1} 3}^{2}}, \quad X_{1-}^{2}=\frac{x_{\overline{3} 2}^{2}}{x_{\overline{3} 1}^{2} x_{\overline{1} 2}^{2}},
$$

which transform as

$$
X_{1 \pm}^{2}{ }^{\prime}=\Omega\left(z_{1}, g\right)^{-2} X_{1 \pm}^{2},
$$

it is obvious, that the quotient of these is invariant under superconformal transformations including R-symmetry and thus is a full invariant:

$$
J_{1}=\frac{X_{1+}^{2}}{X_{1-}^{2}}=\frac{x_{\overline{2} 3}^{2} x_{\overline{3} 1}^{2} x_{\overline{1} 2}^{2}}{x_{\overline{3} 2}^{2} x_{\overline{1} 3}^{2} x_{\overline{2} 1}^{2}} .
$$

This invariant function of three points has the constant 1 as leading term, because the squares of intervals, $x_{i j}^{2}$, become symmetric in their indices, if the Graßmann variables are set to zero. 
We substract this leading constant and define the following nilpotent three point invariant

$$
I_{3 P F}=J_{1}-1
$$

This is more convenient for the expression of the three point function later on, because the third power of $I_{3 P F}$ already vanishes and so a power series with respect to this invariant terminates after second order.

That the degree of $I_{3 P F}$ is three, can be more easily seen, if it is written in another way. In order to do so, we define two Graßmann spinors,

$$
\begin{aligned}
& \Theta_{1}=\mathrm{i}\left(\tilde{\bar{\theta}}_{21} \mathrm{x}_{\overline{2} 1}{ }^{-1}-\tilde{\bar{\theta}}_{31} \mathrm{x}_{\overline{3} 1}{ }^{-1}\right), \\
& \bar{\Theta}_{1}=\mathrm{i}\left(\mathrm{x}_{\overline{1} 3}{ }^{-1} \tilde{\theta}_{13}-\mathrm{x}_{\overline{1} 2}{ }^{-1} \tilde{\theta}_{12}\right) .
\end{aligned}
$$

Lowering the indices we see with eqns. (3.3.67), 3.3.68), 3.3.53), 3.3.58) and (3.3.54), that they transform as

$$
\begin{aligned}
\tilde{\Theta}_{1}^{\prime} & =\left(\Omega_{+}\left(z_{1+}, g\right)\right)^{-\frac{1}{2}}\left(\Upsilon\left(z_{1}, g\right)\right)^{-\frac{1}{3}} \hat{L}^{-1}\left(z_{1}, g\right) \tilde{\Theta}_{1}, \\
\tilde{\bar{\Theta}}_{1}{ }^{\prime} & =\left(\Omega_{-}\left(z_{1-}, g\right)\right)^{-\frac{1}{2}}\left(\Upsilon\left(z_{1}, g\right)\right)^{\frac{1}{3}} \tilde{\bar{\Theta}}_{1} \hat{\bar{L}}^{-1}\left(z_{1}, g\right) .
\end{aligned}
$$

They give a relation between $\tilde{\mathrm{X}}_{1+}$ and $\tilde{\mathrm{X}}_{1-}$ :

$$
\tilde{\mathrm{X}}_{1-}=\tilde{\mathrm{X}}_{1+}-4 \mathrm{i} \bar{\Theta}_{1} \Theta_{1} .
$$

If we plug this into eq. 4.1.7), expand the denominator with the help of eq. A.2.10 and substract 1 again, we find the new expression for $I_{3 P F}$,

$$
I_{3 P F}=-4 \mathrm{i} \frac{\Theta_{1} \mathrm{X}_{1+} \bar{\Theta}_{1}}{X_{1+}^{2}} .
$$

In the construction of $J_{1}$ we first defined $X_{1 \pm}$. In their superconformal transformations the terms from the transformation of the points $z_{2}$ and $z_{3}$ cancel. This, of course, can also be done with the indices cyclically permuted, which would lead to analogously defined $X_{2 \pm}$ and $X_{3 \pm}$. The ratios of all three pairs, $X_{i \pm}{ }^{2}$, are equal to $J_{1}$, which is not effected by the permutation of the indices. Another possibility to construct a three point invariant takes the trace of $X_{1+} \tilde{X}_{1-}$, which is nothing more than the product of the corresponding four-vectors:

$$
-\frac{\operatorname{tr}\left(X_{1+} \tilde{X}_{1-}\right)}{2 \sqrt{X_{1+}{ }^{2} X_{1-}{ }^{2}}}=\frac{X_{1+}{ }^{\mu} X_{1-\mu}}{\sqrt{X_{1+}{ }^{2} X_{1-}{ }^{2}}} .
$$

This can also be expressed in terms of $I_{3 P F}$ :

$$
\frac{X_{1+}{ }^{\mu} X_{1-\mu}}{\sqrt{X_{1+}{ }^{2} X_{1-}}}=1-\frac{1}{2} I_{3 P F} .
$$

It turns out, that there are no ways to construct fully invariant functions of three points of superspace, which are not functions of $I_{3 P F}$. They would still have 
to contain only $X_{1+}, \Theta_{1}$ and $\bar{\Theta}_{1}$ (eqns. 4.1.1), 4.1.9) and 4.1.10) and R-symmetry demands the pairing of the latter two.

But if we skip R-symmetry, two other possibilities open up leading to partial scalar invariants, which we need for the expressions of three-point functions of only chiral and of only anti-chiral superfields.

The contraction of the spinors from eqns. 4.1.9 and 4.1.10 with themselves transform under superconformal symmetry already quite simply:

$$
\begin{aligned}
\Theta_{1}^{\prime} \tilde{\Theta}_{1}^{\prime} & =\left(\Omega\left(z_{1}, g\right)\right)^{-1}\left(\Upsilon\left(z_{1}, g\right)\right)^{-1} \Theta_{1} \tilde{\Theta}_{1} \\
\tilde{\Theta}_{1}^{\prime} \bar{\Theta}_{1}^{\prime} & =\left(\Omega\left(z_{1}, g\right)\right)^{-1} \Upsilon\left(z_{1}, g\right) \tilde{\Theta}_{1} \bar{\Theta}_{1} .
\end{aligned}
$$

With a look on the transformation properties of $X_{1+}^{2}$ in eq. 4.1.6 we can choose a normalization, which eliminates the dependence of the superconformal transformations on the dilations and gives us two partial invariants:

$$
\frac{\Theta_{1} \tilde{\Theta}_{1}}{\sqrt{X_{1+}^{2}}}, \quad \frac{\tilde{\Theta}_{1} \bar{\Theta}_{1}}{\sqrt{X_{1+}^{2}}} .
$$

There is no difference in a possible choice replacing $X_{1+}^{2}$ by $X_{1-}^{2}$ because of eq. (4.1.13). The difference between these two contains $\Theta_{1}$ and $\bar{\Theta}_{1}$, which vanishes in these fractions due to their nilpotency.

The product of these two partial invariants is proportional to the square of $I_{3 P F}$ again.

\subsection{Non-nilpotent four-point invariants}

While the three-point invariants are nilpotent apart from leading constants, the construction of four-point invariants has also to lead to at least two independent non-nilpotent invariants. This can be said before the first calculation within supersymmetry, because these invariants or functions of them have to become ordinary conformal cross ratios, if the Grassmann variables are set to zero. We see, that we only find full non-nilpotent invariants. In section 4.3 we discuss full nilpotent invariants and leave partial R-symmetry violating nilpotent invariants to the appendix C.4.

At first we need to clarify, what we understand as independent non-nilpotent invariants. We regard a set of non-nilpotent invariants to be independent, if there is no function of the invariants in this set and any nilpotent invariants, which is identical zero. Equivalently one can say, that such a set is independent, if the contained invariants with Grassmann variables set to zero are algebraically independent.

Thus there actually cannot be sets with more than two independent non-nilpotent four-point invariants, because there are only two algebraically independent conformal cross ratios. 


\subsubsection{Cross ratios}

In analogy to the conformal case cross ratios can be constructed. The intervals $x_{i j}^{\mu}$ transform homogeneously (eq. (3.3.63) ) and their squares are only left with chiral and anti-chiral factors from dilations:

$$
x_{i j}^{2}{ }^{\prime}=\Omega_{-}\left(z_{i-}, g\right) \Omega_{+}\left(z_{j+}, g\right) x_{i j}^{2} .
$$

With four point there is not only the possibility to cancel these factors in the transformations in ratios like the three point invariant $J_{1}$, but they also drop out in the following cross ratios:

$$
\frac{x_{\bar{r} s}^{2} x_{\bar{t} u}^{2}}{x_{\bar{r} u}^{2} x_{\bar{t} s}^{2}}
$$

They reduce for vanishing Graßmann variables to the ordinary conformal cross ratios. There are six different superconformal cross ratios and their inverses.

We define analogously to eqns. 4.1.1 and 4.1.2

$$
\begin{aligned}
& \tilde{\mathrm{X}}_{1(i-1)+}=-\mathrm{x}_{\overline{1} 4}{ }^{-1} \mathrm{x}_{\bar{i} 4} \mathrm{x}_{\bar{i} 1}^{-1} \\
& \mathrm{X}_{1(i-1)+}=-\tilde{\mathrm{x}}_{\bar{i} 1}^{-1} \tilde{\mathrm{x}}_{\bar{i} 4} \tilde{\mathrm{x}}_{\overline{1} 4}^{-1}, \\
& \tilde{\mathrm{X}}_{1(i-1)-}=\mathrm{x}_{\overline{1} i}^{-1} \mathrm{x}_{\overline{4} i} \mathrm{x}_{\overline{4} 1}{ }^{-1} \\
& \mathrm{X}_{1(i-1)-}=\tilde{\mathrm{x}}_{\overline{4} 1}^{-1} \tilde{\mathrm{x}}_{\overline{4} i} \tilde{\mathrm{x}}_{\overline{1} i}^{-1}
\end{aligned}
$$

$i=2,3$, and analoguosly to 4.1 .5

$$
X_{1(i-1)+}^{2}=\frac{x_{\bar{i} 4}^{2}}{x_{\bar{i} 1}^{2} x_{\overline{1} 4}^{2}}, \quad X_{1(i-1)-}^{2}=\frac{x_{\overline{4} i}^{2}}{x_{\overline{4} 1}^{2} x_{\overline{1} i}^{2}},
$$

with $i=2,3$. The cross ratios can be expressed in terms of the six $X$ 's from eqns. (4.1.5) and 4.2.5). Let us pick out two cross ratios:

$$
\begin{aligned}
& \mathcal{I}_{1}=\frac{x_{\overline{1} 4}^{2} x_{\overline{3} 2}^{2}}{x_{\overline{1} 2}^{2} x_{\overline{3} 4}^{2}}=\frac{X_{1-}{ }^{2}}{X_{1(2)+}{ }^{2}}, \\
& \mathcal{I}_{2}=\frac{x_{\overline{1} 4}^{2} x_{\overline{2} 3}^{2}}{x_{\overline{1} 3}^{2} x_{\overline{2}}^{2}}=\frac{X_{1+}{ }^{2}}{X_{1(1)+}{ }^{2}} .
\end{aligned}
$$

One gets also the nilpotent invariant of three points - ignoring the added constant 1 - with the corresponding indices replaced:

$$
J_{1(i)}=\frac{X_{1(i)+}^{2}}{X_{1(i)-}^{2}},
$$

with $i=1,2$. These relate the cross ratios with each other, so that we can express the other four cross ratios in terms of the two in eqns. 4.2.6) and (4.2.7) and the 
J's:

$$
\begin{aligned}
& \mathcal{I}_{3}=\frac{x_{33}^{2} x_{41}^{2}}{x_{\overline{3} 1}^{2} x_{\overline{4} 2}^{2}}=J_{1}^{-1} J_{1(1)} \mathcal{I}_{2}, \\
& \mathcal{I}_{4}=\frac{x_{25}^{2} x_{\overline{3} 1}^{2}}{x_{\overline{2} 1}^{2} x_{\overline{3} 4}^{2}}=J_{1} \mathcal{I}_{1} \mathcal{I}_{2}^{-1}, \\
& \mathcal{I}_{5}=\frac{x_{2}^{2} x_{41}^{2}}{x_{\overline{2} 1}^{2} x_{\overline{4}}^{2}}=J_{1} J_{1(2)} \mathcal{I}_{1}, \\
& \mathcal{I}_{6}=\frac{x_{13}^{2} x_{42}^{2}}{x_{\overline{1} 2}^{2} x_{\overline{4}}^{2}}=J_{1} J_{1(1)}^{-1} J_{1(2)} \mathcal{I}_{1} \mathcal{I}_{2}^{-1} .
\end{aligned}
$$

There are no other non-nilpotent invariants, which cannot be expressed by $\mathcal{I}_{1}$, $\mathcal{I}_{2}$ and nilpotent invariants, as we will see in the following paragraph.

\subsubsection{Other non-nilpotent invariants}

As we have seen, the construction of the invariants starts from a homogeneously transforming interval, which is $x_{\overline{i j}}$.

There are many possibilities for combinations of the intervals $x_{\bar{i} j}$ for $1 \leq i, j \leq 4$, which are invariant. Still they are built from basic variables, which result from the elimination of the transformation terms.

The variables $X_{1 \pm}$ and $X_{1(i) \pm}$ are already invariant under the transformations of all points but $z_{1}$. Non-nilpotent functions of four points with this property have to be built with these variables and invariants. It is useful for the further calculations to introduce a normalization,

$$
\begin{aligned}
\hat{X}_{1 \pm}^{\mu} & =\frac{X_{1 \pm}^{\mu}}{\left(X_{1(1)+}^{2} X_{1(1)-}^{2}\right)^{\frac{1}{4}}}, \\
\hat{X}_{1(i) \pm}^{\mu} & =\frac{X_{1(i) \pm}^{\mu}}{\left(X_{1(1)+}^{2} X_{1(1)-}^{2}\right)^{\frac{1}{4}}},
\end{aligned}
$$

with $i=1,2$. These are already vectors, which transform as

$$
\begin{aligned}
\left(\hat{X}_{1 \pm}^{\mu}\right)^{\prime} & =\hat{X}_{1 \pm}^{\nu} \hat{R}_{\nu}^{\mu}(z, g) \\
\left(\hat{X}_{1(i) \pm}^{\mu}\right)^{\prime} & =\hat{X}_{1(i) \pm}^{\nu} \hat{R}_{\nu}^{\mu}(z, g)
\end{aligned}
$$

where

$$
\sigma^{\nu} \hat{R}_{\nu}^{\mu}(z, g)=\hat{L}\left(z_{+}, g\right) \sigma_{\mu} \hat{\bar{L}}\left(z_{-}, g\right) .
$$

Hence any contraction of one of these vectors with another is a scalar non-nilpotent invariant. However, we have seen in equation (4.1.13), that the difference between a plus and a minus sign in the indices is only a nilpotent term. Thus the insertion of this or the analogous equation into any of the invariants will give a relation between two non-nilpotent invariants, in which their difference is a nilpotent invariant. 
To write down these relations explicitly we first need to introduce the spinors,

$$
\begin{aligned}
& \tilde{\Theta}_{1(i-1)}=\mathrm{i}\left(\tilde{\mathrm{x}}_{\overline{4} 1}^{-1} \bar{\theta}_{41}-\tilde{\mathrm{x}}_{\bar{i} 1}^{-1} \bar{\theta}_{i 1}\right), \\
& \tilde{\tilde{\Theta}}_{1(i-1)}=\mathrm{i}\left(\theta_{1 i} \tilde{\mathrm{x}}_{\overline{1} i}^{-1}-\theta_{14} \tilde{\mathrm{x}}_{\overline{1} 4}^{-1}\right),
\end{aligned}
$$

analogous to eqns. 4.1.9 and 4.1.10). Furthermore it is also here convenient to introduce a normalization also for all these spinoral functions of three points:

$$
\begin{aligned}
\hat{\Theta}_{1} & =\frac{\Theta_{1}}{\left(X_{1(1)+}^{2} X_{1(1)-}^{2}\right)^{\frac{1}{8}}}, \\
\hat{\bar{\Theta}}_{1} & =\frac{\bar{\Theta}_{1}}{\left(X_{1(1)+}^{2} X_{1(1)-}^{2}\right)^{\frac{1}{8}}}, \\
\hat{\Theta}_{1(i)} & =\frac{\Theta_{1(i)}}{\left(X_{1(1)+}^{2} X_{1(1)-}^{2}\right)^{\frac{1}{8}}}, \\
\hat{\bar{\Theta}}_{1(i)} & =\frac{\bar{\Theta}_{1(i)}}{\left(X_{1(1)+}^{2} X_{1(1)-}^{2}\right)^{\frac{1}{8}}} .
\end{aligned}
$$

Now an example to the mentioned relations can be given compactly: With the equation of $\hat{X}_{1(2)+}^{\mu}$ and $\hat{X}_{1(2)-}^{\mu}$ analogous to eq. 4.1.13 we get the following difference of two non-nilpotent invariants:

$$
\hat{X}_{1(1)+} \cdot \hat{X}_{1(2)-}-\hat{X}_{1(1)+} \cdot \hat{X}_{1(2)+}=2 \mathrm{i} \hat{X}_{1(1)+}^{\mu} \hat{\Theta}_{1} \sigma_{\mu} \hat{\Theta}_{1} .
$$

As there are two invariants on the left hand side of the equation, the right hand side must be a nilpotent invariant. Analogously a couple of similar relations between other non-nilpotent invariants can be written down.

Three contractions of the vector invariants, however, are actually nilpotent apart from a constant leading term. These are $\hat{X}_{1(1) \pm} \cdot \hat{X}_{1(1) \pm}$, which are $\sqrt{J_{1}}, \sqrt{J_{1}^{-1}}$ and a function of $J_{1}$ and another nilpotent invariant.

Moreover $\hat{X}_{1+}^{\mu}, \hat{\Theta}_{1}$ and $\hat{\bar{\Theta}}_{1}$ can be expressed in terms of $\hat{X}_{1(i) \pm}^{\mu}, \hat{\Theta}_{1(i)}$ and $\hat{\bar{\Theta}}_{1(i)}$ :

$$
\begin{aligned}
X_{1+}^{\mu} & =X_{1(1)+}^{\mu}-X_{1(2)-}^{\mu}+2 \mathrm{i} \Theta_{1(1)} \sigma_{\mu} \bar{\Theta}_{1(2)}, \\
\Theta_{1} & =\Theta_{1(1)}-\Theta_{1(2)}, \\
\bar{\Theta}_{1} & =\bar{\Theta}_{1(1)}-\bar{\Theta}_{1(2)} .
\end{aligned}
$$

Eq. 4.1.13 then also determines $\hat{X}_{1-}^{\mu}$. Hence the two invariants,

$$
\left(\hat{X}_{1(2)-}\right)^{2}, \quad \hat{X}_{1(2)-} \cdot \hat{X}_{1(1)+}
$$

are a set of two independent non-nilpotent scalar invariants. All other contractions of the vector invariants in eq. 4.2.13 or functions of these - in fact this means all 


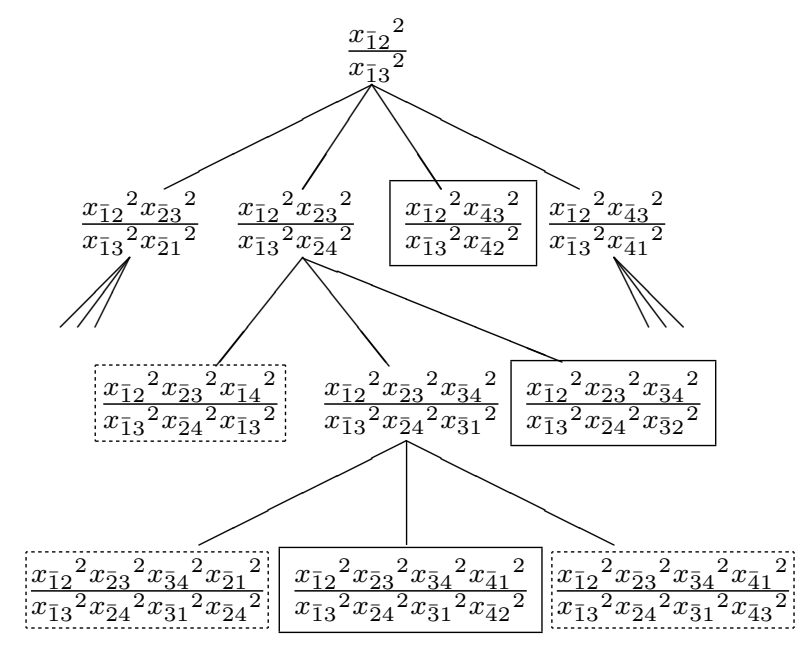

Figure 4.1: A part of the tree starting from $x_{\overline{12}}{ }^{2} / x_{\overline{1} 3}{ }^{2}$ for the construction of invariants as ratios of superconformal intervals. The continuous boxes are the invariants, which end a branch, because they have an index 2 in the interval last added to the denominator. The dashed boxes contain an invariant, which is given by the ratio of two three point functions, $\hat{\mathrm{X}}_{1 \pm}$ and $\hat{\mathrm{X}}_{1(i) \pm}$ and thus end the branch.

scalar non-nilpotent invariants - can be expressed in terms of them and nilpotent invariants. The cross ratios, of course, are no exception because one only has to put hats on all $X$ 's in eqns. 44.2.6), 4.2.7) and (4.2.8). As the $X$ 's appear only in quotients of two of them there, the normalization cancels down. These equations of invariants can be solved for the cross ratios, so that we see, that all non-nilpotent invariants can also be expressed by two cross ratios and nilpotent invariants.

This whole construction building invariant functions can be started in a way, in which analogously to eq. 4.2.13 either $X_{2(i) \pm}, X_{3(i) \pm}$ or $X_{4(i) \pm}$ are defined and so one is left with non-trivial transformations only for the coordinates $z_{2}, z_{3}$ and $z_{4}$, respectively. But this would result in the same calculations with indices permuted. For the cross ratios we have already noted, that a permutation of the indices again gives one of the cross ratios given by 4.2 .2 . We have seen, that all the other non-nilpotent invariants in this section can be expressed in terms of two superconformal cross ratios and nilpotent invariants. Thus a permutation of the indices in these invariants does not lead to invariants, which are independent of those already written down here and the mentioned different ways lead to the same result.

As mentioned above it is already obvious from ordinary conformal invariants, that there can be only two non-nilpotent invariants, which are independent. Here we have seen, how the asymmetry of the superconformal invariants only gives rise to a lot of non-nilpotent invariants related to each other by nilpotent invariants. 
More invariant ratios. An especially interesting subset of non-nilpotent invariants are those, which are ratios of squares of superconformal intervals, $x_{\bar{i} j}{ }^{2}$, or square roots of these ratios. While squares of the variables, $\hat{\mathrm{X}}_{1 \pm}$ and $\hat{\mathrm{X}}_{1(i) \pm}$, their inverses and then, of course, cross ratios belong to it, mixed contractions of the former variables are not of this form.

With 56 invariant ratios of squared superconformal intervals, which themselves can be formed with $\hat{X}_{1 \pm}^{2}$ and $\hat{X}_{1(i) \pm}^{2}$, all other invariant ratios can be formed.

The calculations in Maple, which lead to this result can be found in D.5. In summary one can start with an arbitrary squared superconformal interval - here $x_{\overline{12}}{ }^{2}$ - and devide it in a first step by $x_{\overline{1}}{ }^{2}$ because this cancels the transformation term associated to the index $\overline{1}$. All other starting possibilities are given by permutations of the indices throughout the calculations.

One continues to multiply alternately to the numerator and the denominator the possible intervals, which just eliminate the transformation terms of the last added factor, which has not yet been eliminated, but do not cancel down with other intervals in the ratio. The list of possible terms forms a tree and this tree grows despite of the terminating condition for single branches: A new term in the denominator, which just has 2 as second index, ends a branch.

Further terminating conditions are added to get to the goal. The variables $\hat{X}_{1 \pm}^{2}$ and $\hat{X}_{1(i) \pm}^{2}$ can only appear in pairs with their inverses. So the hats cancels. Whenever any variable $X_{1 \pm}^{2}$ or $X_{1(i) \pm}^{2}$ appears together with any of the inverses in the ratio of a branch, it is terminated. Although part of this ratio is not expressed by these variables, nothing is missed, as this part appears in all possible other combinations in other branches (possibly belonging to a tree with permuted indices). This condition does not lead to invariants, but prevents endless loops. A part of the tree starting from $x_{\overline{12}}{ }^{2} / x_{\overline{13}}{ }^{2}$ is depicted in figure 4.1 .

The simplest of the ends of branches are the appearances of cross ratios. At the end there are seven branches, which end with the first terminating condition and so with finished invariant ratios in this tree.

$$
\begin{aligned}
& \frac{x_{\overline{1} 2}^{2} x_{\overline{4} 3}^{2}}{x_{\overline{1} 3}^{2} x_{\overline{4} 2}^{2}}=\frac{\hat{X}_{1(2)-}^{2}}{\hat{X}_{1(1)-}^{2}}, \\
& \frac{x_{\overline{1} 2}^{2} x_{\overline{2} 3}^{2} x_{\overline{3} 1}^{2}}{x_{\overline{1} 3}{ }^{2} x_{\overline{2} 1}{ }^{2} x_{\overline{3} 2}{ }^{2}}=\frac{\hat{X}_{1+}^{2}}{\hat{X}_{1-}^{2}}, \\
& \frac{x_{\overline{12}}^{2} x_{\overline{2} 3}^{2} x_{\overline{4} 1}^{2}}{x_{\overline{1} 3}{ }^{2} x_{\overline{2} 1}{ }^{2} x_{\overline{4} 2}{ }^{2}}=\frac{\hat{X}_{1+}^{2}}{\hat{X}_{1(1)-}^{2}}, \\
& \frac{x_{\overline{1} 2}^{2} x_{\overline{2} 3}^{2} x_{\overline{3} 4}^{2}}{x_{\overline{1} 3}{ }^{2} x_{\overline{2}}{ }^{2} x_{\overline{3} 2}{ }^{2}}=\frac{\hat{X}_{1+}^{2} \hat{X}_{1(2)+}^{2}}{\hat{X}_{1(1)+}^{2} \hat{X}_{1-}^{2}}, \\
& \frac{x_{\overline{1} 2}^{2} x_{\overline{4} 3}^{2} x_{\overline{3} 1}^{2}}{x_{\overline{1} 3}{ }^{2} x_{\overline{4} 1}{ }^{2} x_{\overline{3} 2}{ }^{2}}=\frac{\hat{X}_{1(2)-}^{2}}{\hat{X}_{1-}^{2}},
\end{aligned}
$$




$$
\begin{aligned}
& \frac{x_{\overline{1} 2}{ }^{2} x_{\overline{2} 3}{ }^{2} x_{\overline{3} 4}{ }^{2} x_{\overline{4} 1}{ }^{2}}{x_{\overline{1} 3}{ }^{2} x_{\overline{2} 4}{ }^{2} x_{\overline{3} 1}{ }^{2} x_{\overline{4} 2}{ }^{2}}=\frac{\hat{X}_{1+}^{2} \hat{X}_{1(2)+}^{2}}{\hat{X}_{1(1)+}^{2} \hat{X}_{1(1)-}^{2}}, \\
& \frac{x_{\overline{1} 2}^{2} x_{\overline{4} 3}^{2} x_{\overline{2} 1}^{2} x_{\overline{34}}^{2}}{x_{\overline{1} 3}{ }^{2} x_{\overline{4} 1}{ }^{2} x_{\overline{2} 4}{ }^{2} x_{\overline{3} 2}{ }^{2}}=\frac{\hat{X}_{1(2)-}^{2} \hat{X}_{1(2)+}^{2}}{\hat{X}_{1(1)+}^{2} \hat{X}_{1-}^{2}} .
\end{aligned}
$$

The second one is $J_{1}$ and so essentially a nilpotent invariant.

As all have the form of ratios of the variables $\hat{X}_{1 \pm}^{2}$ and $\hat{X}_{1(i) \pm}^{2}$, this shows together with the permuted trees from the other starting possibilities, that all invariant ratios of squared superconformal intervals can be expressed in this form.

At last in this section we want to give the following relations of these three-point variables to cross ratio, $J_{1}$ and $J_{1(i)}$ :

$$
\begin{aligned}
& \hat{X}_{1+}^{2}=\sqrt{\frac{x_{\overline{1} 2}^{2} x_{\overline{4} 1}^{2} x_{\overline{1} 4}^{2} x_{\overline{2} 3}^{4}}{x_{\overline{2} 4}{ }^{2} x_{\overline{4} 2}{ }^{2} x_{\overline{2} 1}{ }^{2} x_{\overline{13}}{ }^{4}}}=\sqrt{\frac{\mathcal{I}_{2} \mathcal{I}_{5}}{\mathcal{I}_{6}}}=\sqrt{J_{1(1)}} \mathcal{I}_{2}, \\
& \hat{X}_{1-}^{2}=\sqrt{\frac{x_{\overline{2} 1}^{2} x_{\overline{4} 1}^{2} x_{\overline{1} 4}^{2} x_{\overline{3} 2}^{4}}{x_{\overline{2} 4}{ }^{2} x_{\overline{4} 2}{ }^{2} x_{\overline{1} 2}{ }^{2} x_{\overline{3} 1}{ }^{4}}}=\sqrt{\frac{\mathcal{I}_{1} \mathcal{I}_{3}}{\mathcal{I}_{4}}}=\sqrt{J_{1(1)}} J_{1}{ }^{-1} \mathcal{I}_{2}, \\
& \hat{X}_{1(1)+}^{2}=\sqrt{\frac{x_{\overline{1} 2}^{2} x_{\overline{41}}^{2} x_{\overline{2}}{ }^{2}}{x_{\overline{4} 2}{ }^{2} x_{\overline{2} 1}{ }^{2} x_{\overline{1}}{ }^{2}}}=\sqrt{J_{1(1)}} \text {, }
\end{aligned}
$$

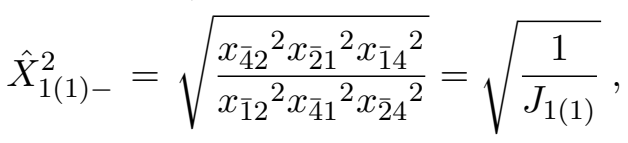

$$
\begin{aligned}
& \hat{X}_{1(2)+}^{2}=\sqrt{\frac{x_{\overline{2} 1}{ }^{2} x_{\overline{4} 1}{ }^{2} x_{\overline{1} 2}{ }^{2} x_{\overline{3} 4}{ }^{4}}{x_{\overline{2} 4^{2}}{ }^{2} x_{\overline{4} 2}{ }^{2} x_{\overline{1}}{ }^{2} x_{\overline{3} 1}{ }^{4}}}=\sqrt{\frac{\mathcal{I}_{3}}{\mathcal{I}_{1} \mathcal{I}_{4}}}=\sqrt{J_{1(1)}} J_{1}{ }^{-1} \mathcal{I}_{1}{ }^{-1} \mathcal{I}_{2}, \\
& \hat{X}_{1(2)-}^{2}=\sqrt{\frac{x_{\overline{2} 1}^{2} x_{\overline{1} 2}^{2} x_{\overline{1} 4}^{2} x_{\overline{4} 3}^{4}}{x_{\overline{2} 4}{ }^{2} x_{\overline{4} 2}{ }^{2} x_{\overline{4} 1}{ }^{2} x_{\overline{1} 3}{ }^{4}}}=\sqrt{\frac{\mathcal{I}_{2}}{\mathcal{I}_{5} \mathcal{I}_{6}}}=\sqrt{J_{1(1)}} J_{1}{ }^{-1} J_{1(2)}{ }^{-1} \mathcal{I}_{1}{ }^{-1} \mathcal{I}_{2} \text {. }
\end{aligned}
$$

These equations are very useful to find bugs in procedures calculating with these entities.

\subsection{Nilpotent four-point invariants}

In the last section we already stumbled over a few nilpotent invariants of four points. Here we construct full nilpotent invariants systematically. The rest of the partial invariants is left to appendix C.4.

We establish a set of invariants, which generates all full four-point invariants. This set contains ten independent full invariants - two non-nilpotent and eight nilpotent -, which is a significant reduction compared to the number in [Par99], where for $\mathcal{N}=1$ sixteen invariants have been counted as presumably independent. For this result we show, that the additional six invariants in [Par99], which are of 
higher order in Graßmann variables than the other eight nilpotent invairants, can be expressed by our set of ten invariants.

Then dependencies of monomials of the eight nilpotent invariants are investigated. This leads to a set of monomials, which is some kind of basis for full invariants, if we allow the coefficients to be functions of non-nilpotent invariants. In the discussion of these monomials the independence of the eight nilpotent invariants, which appear here, of course, as the monomials of degree one, is shown, which establishes the minimality of the whole generating set of ten invariants mentioned above.

\subsubsection{Construction}

The nilpotent invariants may contain $\hat{\tilde{\Theta}}_{1(j)}$ and $\hat{\tilde{\Theta}}_{1(j)}$ (cf. eqns. 4.2.18) and (4.2.19), for which only the superconformal transformations of $z_{1}$ are non-trivial. Because of eqns. (3.3.67) and (3.3.68) they transform as

$$
\begin{aligned}
& \hat{\tilde{\Theta}}_{1(j)}^{\prime}=\left(\frac{\Omega\left(z_{1}, g\right)}{\Omega_{+}\left(z_{1+}, g\right)}\right)^{\frac{1}{2}} \hat{L}^{-1}\left(z_{1+}, g\right) \hat{\tilde{\Theta}}_{1(j)} u\left(z_{1}, g\right) \\
& \hat{\tilde{\Theta}}_{1(j)}^{\prime}=\left(\frac{\Omega\left(z_{1}, g\right)}{\Omega_{-}\left(z_{1-}, g\right)}\right)^{\frac{1}{2}} u^{-1}\left(z_{1}, g\right) \hat{\tilde{\Theta}}_{1(j)} \hat{\bar{L}}^{-1}\left(z_{1-}, g\right) .
\end{aligned}
$$

If we consider eqns. 4.2.16 together with eq. 4.2.17), we see, that we can build invariants with $\hat{\tilde{\Theta}}_{1(j)}, \hat{\tilde{\Theta}}_{1(j)}$ and $\tilde{\mathrm{X}}_{1(i) \pm}$. There have to be an equal number of $\hat{\tilde{\Theta}}_{1(j)}$ and $\hat{\tilde{\Theta}}_{1(j)}$, so that the factors from dilatation and R-symmetry drop out. Then only all indices have to be contracted to get a scalar nilpotent invariant. In other words we get a generating set of the nilpotent invariants, if we look at all possible contractions of these spinors and spinoral matrices, such that there is the same number of contractions of dotted and undotted indices. In the following we see, that all of these contractions are functions of only ten invariants, of which eight are nilpotent.

There are many possible sets of eight independent nilpotent invariants one can choose. Here the choice is the set of all possible contractions of $\hat{\Theta}_{1(j)}, \hat{\bar{\Theta}}_{1(j)}, \hat{\mathrm{X}}_{1(1)+}$ and $\hat{\mathrm{X}}_{1(2)-}$ with lowest non-vanishing order $\mathcal{O}(\theta \bar{\theta})$ in Graßmann variables:

$$
\begin{aligned}
& I_{1 i j}=\hat{X}_{1(1)+}^{\mu} \hat{\Theta}_{1(i)} \sigma_{\mu} \hat{\bar{\Theta}}_{1(j)}=-\hat{\tilde{\tilde{\Theta}}}_{1(j)} \hat{\tilde{X}}_{1(1)+} \hat{\tilde{\Theta}}_{1(i)}, \\
& I_{2 i j}=\hat{X}_{1(2)-}^{\mu} \hat{\Theta}_{1(i)} \sigma_{\mu} \hat{\bar{\Theta}}_{1(j)}=-\hat{\tilde{\Theta}}_{1(j)} \hat{\tilde{X}}_{1(2)-} \hat{\tilde{\Theta}}_{1(i)},
\end{aligned}
$$

with $i, j=1,2$ and

$$
\begin{aligned}
& \hat{\tilde{\mathrm{X}}}_{1(1)+}=\frac{-\mathrm{x}_{\overline{1} 4}{ }^{-1} \mathrm{x}_{\overline{2} 4} \mathrm{x}_{\overline{2} 1}-1}{\left(X_{1(1)+}^{2} X_{1(1)-}^{2}\right)^{\frac{1}{4}}}, \\
& \hat{\tilde{\mathrm{X}}}_{1(2)-}=\frac{\mathrm{x}_{\overline{1} 3}^{-1} \mathrm{x}_{\overline{4} 3 \mathrm{x}_{\overline{4} 1}-1}}{\left(X_{1(1)+}^{2} X_{1(1)-}^{2}\right)^{\frac{1}{4}}} .
\end{aligned}
$$


Next we want to express one of these invariants, $I_{111}$, as an example, in terms of the supersymmetric intervals. Together with $I_{222}$ and a combination of all $I_{i j k}$, which we will find in section 4.3 .3 to be related to the three point invariant discussed above, it belongs to a group of invariants, which is especially relevant for the next chapter. They also have a particularly simple structure because except for their normalization they contain only the variables of three points in superspace . $I_{111}$ even contains in the numerator and the denominator only the variables of the points $z_{1}, z_{2}$ and $z_{4}$. Still it has its correct place here among the four point invariants because the normalization is exchangeable, for example, by any other square root of $X_{i(j)+}^{2} X_{i(j)-}^{2}$, which all have the same transformation properties. Furthermore it is needed for completeness.

We plug eqns. 4.3.5), 4.2.18) and 4.2.19) into $I_{111}$ (eq. 4.3.3) and multiply for simplicity by the normalisation:

$$
\sqrt{X_{1(1)+}^{2} X_{1(1)-}^{2}} I_{111}=-\left(\theta_{12} \tilde{\mathrm{x}}_{\overline{1} 2}^{-1}-\theta_{14} \tilde{\mathrm{x}}_{\overline{1} 4}^{-1}\right) \mathrm{x}_{\overline{1} 4}{ }^{-1} \mathrm{x}_{\overline{2} 4} \mathrm{x}_{\overline{2} 1}{ }^{-1}\left(\tilde{\mathrm{x}}_{\overline{4} 1}^{-1} \bar{\theta}_{41}-\tilde{\mathrm{x}}_{\overline{2} 1}^{-1} \bar{\theta}_{21}\right) \text {. }
$$

When we expand the brackets, three of four terms shorten already because the matrices appearing here square to a scalar times the identity matrix (cf. eqns. A.2.1 and (3.2.23). With the help of eqns. A.2.2 and A.2.4 we can break down the remaining products. We gets

$$
\begin{aligned}
& \sqrt{X_{1(1)+}^{2} X_{1(1)-}^{2}} I_{111} \\
& =-\frac{x_{\overline{2} 4}{ }^{2}}{x_{\overline{2} 1}^{2} x_{\overline{1} 4}^{2}}\left[\theta_{12} \tilde{x}_{\overline{2} 4}^{-1} \bar{\theta}_{41}-4 \mathrm{i} \theta_{12} \tilde{x}_{\overline{1} 2}^{-1} \bar{\theta}_{12} \theta_{12} \tilde{x}_{\overline{4} 1}^{-1} \bar{\theta}_{41}-4 \mathrm{i} \theta_{12} \tilde{x}_{\overline{2} 4}^{-1} \bar{\theta}_{24} \theta_{41} \tilde{x}_{\overline{4} 1}^{-1} \bar{\theta}_{41}\right. \\
& -4 \mathrm{i} \theta_{12} \tilde{x}_{\overline{1} 2}^{-1} \bar{\theta}_{12} \theta_{24} \tilde{x}_{\overline{2} 4}^{-1} \bar{\theta}_{41}-4 \mathrm{i} \theta_{12} \tilde{x}_{\overline{1} 2}^{-1} \bar{\theta}_{12} \theta_{24} \tilde{x}_{\overline{4} 1}^{-1} \bar{\theta}_{41} \\
& -16 \theta_{12} \tilde{x}_{\overline{1} 2}^{-1} \bar{\theta}_{12} \theta_{24} \tilde{x}_{\overline{2} 4}^{-1} \bar{\theta}_{24} \theta_{41} \tilde{x}_{\overline{4} 1}^{-1} \bar{\theta}_{41} \\
& -\theta_{12} \tilde{x}_{\overline{1} 2}^{-1} \bar{\theta}_{21}-\theta_{12} \tilde{x}_{\overline{2} 4}^{-1} \bar{\theta}_{21}+4 \mathrm{i} \theta_{12} \tilde{x}_{\overline{1} 2}^{-1} \bar{\theta}_{12} \theta_{24} \tilde{x}_{\overline{2} 4}^{-1} \bar{\theta}_{21} \\
& +\theta_{14} \tilde{x}_{\overline{2} 4}^{-1} \bar{\theta}_{21} \\
& \left.-\theta_{14} \tilde{x}_{\overline{4} 1}^{-1} \bar{\theta}_{41}-\theta_{14} \tilde{x}_{\overline{2} 4}^{-1} \bar{\theta}_{41}+4 \mathrm{i} \theta_{14} \tilde{x}_{\overline{2} 4}^{-1} \bar{\theta}_{24} \theta_{41} \tilde{x}_{\overline{4} 1}^{-1} \bar{\theta}_{41}\right]
\end{aligned}
$$

The summands are still grouped corresponding to the terms they originate from, which is also visible from the respective first interval $\theta_{i j}$ and last interval $\bar{\theta}_{i j}$, which stayed unchanged.

Now we switch indices in some intervals, so that always the pair 41 and not 14 appears and for all other pairs the first index is the lower one. This sorting rule is also often applied later on. We find, that the group of four terms $\theta_{i j} \tilde{x}_{\overline{2} 4}^{-1} \bar{\theta}_{k l}$ can be pulled together and gives $\theta_{24} \tilde{x}_{\overline{2} 4}^{-1} \bar{\theta}_{24}$. Also the higher order terms can be simplified, so that we can express the large bracket only by

$$
\rho_{i j}=\theta_{i j} \tilde{x}_{\overline{i j}}^{-1} \bar{\theta}_{i j}
$$


At last we divide by the normalisation and get

$$
\begin{aligned}
& I_{111}=\sqrt{\frac{x_{\overline{2} 4}^{2} x_{\overline{1} 2}^{2} x_{\overline{4} 1}^{2}}{x_{\overline{4} 2}^{2} x_{\overline{2} 1}^{2} x_{\overline{1} 4}^{2}}}\left[-\rho_{41}-\rho_{12}-\rho_{24}\right. \\
& -4 \mathrm{i}\left(\rho_{12} \rho_{41}+\rho_{12} \rho_{24}+\rho_{24} \rho_{41}\right) \\
& \left.+16 \rho_{12} \rho_{24} \rho_{41}\right] \text {. }
\end{aligned}
$$

The square root can also be written in terms of $\rho$ 's with eq. A.2.11. If we plug this in for the three factors in the denominator, the superconformal intervals cancel and only the following product is left in the radicand, which is $J_{1(1)}$ :

$$
J_{1(1)}=\left(1+4 \mathrm{i} \rho_{24}\right)\left(1+4 \mathrm{i} \rho_{12}\right)\left(1+4 \mathrm{i} \rho_{41}\right) .
$$

The square root of it is

$$
\begin{aligned}
\sqrt{\frac{x_{\overline{2} 4}^{2} x_{\overline{1} 2}^{2} x_{\overline{4} 1}^{2}}{x_{\overline{4} 2}^{2} x_{\overline{2} 1}^{2} x_{\overline{1} 4}^{2}}} & =X_{1(1)+}{ }^{2}=\sqrt{J_{1(1)}} \\
& =\left(1+2 \mathrm{i} \rho_{24}+2 \rho_{24}{ }^{2}\right)\left(1+2 \mathrm{i} \rho_{12}+2 \rho_{12}{ }^{2}\right)\left(1+2 \mathrm{i} \rho_{41}+2 \rho_{41}{ }^{2}\right)
\end{aligned}
$$

If we plug this into eq. 4.3.10, we get

$$
\begin{aligned}
I_{111}= & -\rho_{41}-\rho_{12}-\rho_{24}+2 \mathrm{i}\left(\rho_{41}^{2}+\rho_{12}^{2}+\rho_{24}^{2}+4 \rho_{24} \rho_{12}+4 \rho_{41} \rho_{12}+4 \rho_{24} \rho_{41}\right) \\
& +10\left(\rho_{24}^{2} \rho_{12}+\rho_{24} \rho_{12}^{2}+\rho_{24}^{2} \rho_{41}+\rho_{24} \rho_{41}^{2}+\rho_{41}^{2} \rho_{12}+\rho_{41} \rho_{12}^{2}\right)+52 \rho_{24} \rho_{12} \rho_{12} \\
& -8 \mathrm{i}\left(\rho_{24}^{2} \rho_{12}^{2}+\rho_{41}^{2} \rho_{12}^{2}+\rho_{24}^{2} \rho_{41}^{2}+7 \rho_{12} \rho_{24} \rho_{41}^{2}+7 \rho_{12} \rho_{24}^{2} \rho_{41}+7 \rho_{12}^{2} \rho_{24} \rho_{41}\right)
\end{aligned}
$$

which we simplify in the following significantly until we get the expression in eq. 4.3.18). It is already considered, that not only cubes of the $\rho$ 's, but also products like $\rho_{12} \rho_{24}^{2} \rho_{41}^{2}$ vanish (the latter because $\theta_{12}=-\theta_{24}-\theta_{41}$ ).

From the study of the third and fourth power of $I_{111}$, which vanish, one can expect further equations of combinations of $\rho$ 's, which vanish, and simplify the expression without expanding all $\rho$ 's.

We start with the fourth power (cf. eq. 4.3.10),

$$
\begin{aligned}
I_{111}^{4} & =6 \rho_{24}^{2} \rho_{12}^{2}+6 \rho_{41}^{2} \rho_{12}^{2}+6 \rho_{24}^{2} \rho_{41}^{2}+12 \rho_{12} \rho_{24} \rho_{41}^{2}+12 \rho_{12} \rho_{24}^{2} \rho_{41}+12 \rho_{12}^{2} \rho_{24} \rho_{41} \\
& =0 .
\end{aligned}
$$

This eliminates already three fourth order terms in eq. 4.3.13. We implemented this and the following identities, which we retrieved this way, in Maple with the help of the function "algsubs", which substitutes algebraic expression. It allows to implement all identities, which result from nilpotency, without the effort to implement the nilpotency itself. The term, to which it is applied, should only have been expanded 
before. Thus in every of the following steps all of the identities listed before that step are already used.

The next one regards the third power. But this is a rather long expression, which we can write down in a more illustrative way with the help of two other identities. For one of those we multiply it by $\rho_{12}$, which still has to be zero:

$$
I_{111}{ }^{3} \rho_{12}=3 \rho_{24}^{2} \rho_{41}^{2}+3 \rho_{12} \rho_{24} \rho_{41}^{2}+3 \rho_{12} \rho_{24}^{2} \rho_{41}=0 .
$$

Similarly the product with $\rho_{41}$ is

$$
I_{111}^{3} \rho_{41}=-3 \rho_{12}^{2} \rho_{41}^{2}-3 \rho_{12} \rho_{24} \rho_{41}^{2}-3 \rho_{12}^{2} \rho_{24} \rho_{41}=0 .
$$

The third possibility of such a product with $\rho_{24}$ is already zero with these conditions.

Looking at the third power we notice, that one can use eq. A.2.12 to get a shorter expression:

$$
\begin{aligned}
I_{111}^{3}= & -3 \rho_{24}^{2} \rho_{12}-3 \rho_{24} \rho_{12}^{2}-3 \rho_{24}^{2} \rho_{41}-6 \rho_{12} \rho_{24} \rho_{41}-3 \rho_{12}^{2} \rho_{41}-3 \rho_{41}^{2} \rho_{24} \\
& -3 \rho_{41}^{2} \rho_{12}+12 \mathrm{i} \rho_{41}^{2} \rho_{24}^{2}+12 \mathrm{i} \rho_{12} \rho_{24} \rho_{41}^{2}+12 \mathrm{i} \rho_{12}^{2} \rho_{41}^{2} \\
= & -3 \rho_{24}^{2} \rho_{12}-3 \rho_{24} \rho_{12}^{2}+3 \rho_{24}^{2} \rho_{14}+6 \rho_{12} \rho_{24} \rho_{14}+3 \rho_{12}^{2} \rho_{14} \\
& -3 \rho_{14}^{2} \rho_{24}-3 \rho_{14}^{2} \rho_{12} \\
= & 0 .
\end{aligned}
$$

Note, that $\rho_{41}^{2}=\rho_{14}^{2}$. For the implementation of this condition in the computer algebra worksheet to be consistent with our previous steps, we have used the first longer expression. Alternatively one can also take eq. A.2.12.

At this point we also get the result for $I_{111}$, which is certainly not subject to any vanishing higher degree terms, because only one of these has not already disappeared.

Corollary. The invariant $I_{111}$ can be expressed with $\rho$ 's (cf. eq. (4.3.9) alone:

$$
\begin{aligned}
I_{111}= & -\rho_{41}-\rho_{12}-\rho_{24}+2 \mathrm{i}\left(\rho_{41}^{2}+\rho_{12}{ }^{2}+\rho_{24}{ }^{2}+4 \rho_{24} \rho_{12}+4 \rho_{41} \rho_{12}+4 \rho_{24} \rho_{41}\right) \\
& +32 \rho_{41} \rho_{12} \rho_{24} .
\end{aligned}
$$

The other nilpotent invariants, $I_{i j k}$, can not be written in terms of $\rho$ 's alone, as there is no cancellation with the normalization, which here led to the factor $X_{1(1)+}{ }^{2}$ in eq. 4.3.10. In appendix C.1 we list the lowest order terms of these invariants. There we see, that already to this order most of the other invariants are significantly more complicated than $I_{111}$.

Other possible contractions of $\hat{\Theta}_{1(i)}$ and $\hat{\bar{\Theta}}_{1(j)}$. How can the other possible contractions mentioned above be expressed in terms of the invariants $I_{i j k}$ and two non-nilpotent invariants? The other nilpotent invariants with the leading term of order $\theta \bar{\theta}$ are contractions with $\hat{\mathrm{X}}_{1(1)-}$ and $\hat{\mathrm{X}}_{1(2)+}$. But in analogy to eq. 4.1.13 these two matrices differ only in higher order of Grassmann variables, so that we find, that

$$
\begin{aligned}
& \hat{\tilde{\Theta}}_{1(j)} \hat{\tilde{X}}_{1(1)-} \hat{\tilde{\Theta}}_{1(i)}=I_{1 i j}-4 i \hat{\tilde{\tilde{\Theta}}}_{1(j)} \hat{\bar{\Theta}}_{1(1)} \hat{\Theta}_{1(1)} \hat{\tilde{\Theta}}_{1(i)}, \\
& \hat{\tilde{\tilde{\Theta}}}_{1(j)} \hat{\tilde{\mathrm{X}}}_{1(2)+} \hat{\tilde{\Theta}}_{1(i)}=I_{2 i j}+4 \mathrm{i} \hat{\tilde{\Theta}}_{1(j)} \hat{\bar{\Theta}}_{1(2)} \hat{\Theta}_{1(2)} \hat{\tilde{\Theta}}_{1(i)} .
\end{aligned}
$$


In order to get to the promised result and trace all other nilpotent invariants back to the chosen ones, we need to express nilpotent invariants with leading term of order $(\theta \bar{\theta})^{2}$, which were still regarded to be independent in [Par99], in terms of these eight.

This is one of the steps, which only work for $\mathcal{N}=1$. The properties of the spinors in eqns. 3.2 .12 and 3.2 .13 propagate to similar properties of the spinoral functions of three points in these invariants:

$$
\begin{aligned}
& \hat{\Theta}_{1(j)}^{\alpha} \hat{\Theta}_{1(j)}^{\beta}=-\frac{1}{2}\left(\epsilon^{-1}\right)^{\alpha \beta} \hat{\Theta}_{1(j)} \hat{\tilde{\Theta}}_{1(j)}, \\
& \hat{\bar{\Theta}}_{1(j)}^{\dot{\alpha}} \hat{\bar{\Theta}}_{1(j)}^{\dot{\beta}}=\frac{1}{2}\left(\bar{\epsilon}^{-1}\right)^{\dot{\alpha} \dot{\beta}} \hat{\tilde{\Theta}}_{1(j)} \hat{\bar{\Theta}}_{1(j)} .
\end{aligned}
$$

Together with the properties of the sigma matrices we find, that most of the nilpotent invariants with leading order $(\theta \bar{\theta})^{2}$ are just products of the chosen eight nilpotent invariants. To see this we start with the products. Eq. 4.3.21) can be used in products of two invariants with the same spinor $\hat{\Theta}_{1(j)}$.

$$
\begin{aligned}
I_{1 j i} I_{1 j k} & =\hat{X}_{1(1)+}^{\mu} \hat{\Theta}_{1(j)} \sigma_{\mu} \hat{\Theta}_{1(i)} \hat{X}_{1(1)+}^{\nu} \hat{\Theta}_{1(j)} \sigma_{\nu} \hat{\bar{\Theta}}_{1(k)} \\
& =\frac{1}{2} \hat{\Theta}_{1(j)} \hat{\tilde{\Theta}}_{1(j)} \hat{X}_{1(1)+}^{\mu} \hat{X}_{1(1)+}^{\nu} \hat{\tilde{\Theta}}_{1(i)} \bar{\sigma}_{\mu} \sigma_{\nu} \hat{\bar{\Theta}}_{1(k)} \\
& =\frac{1}{2} \hat{X}_{1(1)+}^{2} \hat{\Theta}_{1(j)} \hat{\tilde{\Theta}}_{1(j)} \hat{\tilde{\Theta}}_{1(i)} \hat{\bar{\Theta}}_{1(k)} .
\end{aligned}
$$

For the last step we use eq. A.1.9). If this equation is contracted with a symmetric tensor $Y^{\mu \nu}$, one can relabel the indices in one of the summands and gets

$$
Y^{\mu \nu} \bar{\sigma}_{\mu} \sigma_{\nu}=Y_{\mu}^{\mu} 1_{2 \times 2} .
$$

With this applied we essentially end up with the desired invariants with leading order $(\theta \bar{\theta})^{2}$. An analogous calculation gives those invariants with twice the same $\hat{\bar{\Theta}}_{1(j)}$ :

$$
\hat{\Theta}_{1(i)} \hat{\tilde{\Theta}}_{1(k)} \hat{\tilde{\tilde{\Theta}}}_{1(j)} \hat{\bar{\Theta}}_{1(j)}=\frac{2}{\hat{X}_{1(1)+}^{2}} I_{1 i j} I_{1 k j}
$$

As the contractions are commutative, we have eight of nine possible combinations of the four contracted spinors $\hat{\Theta}_{1(i)} \hat{\tilde{\Theta}}_{1(k)} \hat{\tilde{\tilde{\Theta}}}_{1(j)} \hat{\bar{\Theta}}_{1(l)}$. For the ninth we can use neither eq. 4.3.21 nor eq. 4.3.22 because there are no fitting pairs. Still it is equal to a combination of four nilpotent invariants from our selection:

$$
\hat{\Theta}_{1(1)} \hat{\tilde{\Theta}}_{1(2)} \hat{\tilde{\Theta}}_{1(1)} \hat{\bar{\Theta}}_{1(2)}=\frac{1}{\hat{X}_{1(1)+}}\left(I_{111} I_{122}+I_{112} I_{121}\right) .
$$

This equation can be shown rewriting the bracket on the right-hand side:

$$
\begin{aligned}
& \left(I_{111} I_{122}+I_{112} I_{121}\right) \\
= & \hat{X}_{1(1)+}^{\mu} \hat{X}_{1(1)+}^{\nu}\left(\hat{\Theta}_{1(1)} \sigma_{\mu} \hat{\bar{\Theta}}_{1(1)} \hat{\Theta}_{1(2)} \sigma_{\nu} \hat{\bar{\Theta}}_{1(2)}+\hat{\Theta}_{1(1)} \sigma_{\mu} \hat{\bar{\Theta}}_{1(2)} \hat{\Theta}_{1(2)} \sigma_{\nu} \hat{\bar{\Theta}}_{1(1)}\right) \\
= & \hat{X}_{1(1)+}^{\mu} \hat{X}_{1(1)+}^{\nu} \hat{\Theta}_{1(1)}^{\alpha} \hat{\bar{\Theta}}_{1(1)}^{\dot{\alpha}} \hat{\Theta}_{1(2)}^{\beta} \hat{\bar{\Theta}}_{1(2)}^{\dot{\beta}}\left(\sigma_{\mu_{\alpha \dot{\alpha}}} \sigma_{\nu \beta \dot{\beta}}-\sigma_{\mu_{\alpha \dot{\beta}}} \sigma_{\nu \beta \dot{\alpha}}\right) .
\end{aligned}
$$


Remark. With eqns. (4.3.23), 4.3.25) and 4.3.27) we have expressed all six invariants of the form $\hat{\Theta}_{1(i)} \hat{\tilde{\Theta}}_{1(j)} \hat{\tilde{\Theta}}_{1(k)} \hat{\bar{\Theta}}_{1(l)}$, which have been counted in [Par99] as persumably independent, in terms of the invariants, $I_{i j k}$, and non-nilpotent invariants.

One can show even more with the following property of sigma matrices.

Lemma 4.1. Let $Y^{\mu \nu}$ be an arbitrary symmetric tensor. With the Pauli matrices, $\sigma_{\mu}$,

$$
Y^{\mu \nu}\left(\sigma_{\mu_{\alpha \dot{\alpha}}} \sigma_{\nu \dot{\beta}}-\sigma_{\mu_{\alpha \dot{\beta}}} \sigma_{\nu \beta \dot{\alpha}}\right)=Y^{\mu}{ }_{\mu} \epsilon_{\alpha \beta} \bar{\epsilon}_{\dot{\alpha} \dot{\beta}}
$$

Proof. The combination of sigma matrices in the brackets times the symmetric tensor is anti-symmetric in the permutation of the two undotted indices as well as the two dotted indices. In consequence it can only be non-zero for $\alpha \neq \beta$ and $\dot{\alpha} \neq \dot{\beta}$.

So the remaining terms have only summands in the bracket, which are of one of the following four forms:

$$
\sigma_{\mu_{1 \dot{1}}} \sigma_{\nu_{2} \dot{2}} \quad \sigma_{\mu_{1} \dot{2}} \sigma_{\nu_{2} \dot{1}} \quad \sigma_{\mu_{21} \dot{1}} \sigma_{\nu_{1} \dot{2}} \quad \sigma_{\mu_{2 \dot{2}}} \sigma_{\nu_{11}}
$$

Thus these terms only do not vanish, if the two Pauli matrices in each of these products are either both diagonal $\left(\sigma_{0}\right.$ and $\left.\sigma_{3}\right)$ or both antidiagonal $\left(\sigma_{1}\right.$ and $\left.\sigma_{2}\right)$ and only one of the summands in the bracket survives. Of a total of 512 summands in all components in eq. (4.3.28 there 32 summands left at this point. Let us look at this point only at the component with $\alpha=\dot{\alpha}=1$ and $\beta=\dot{\beta}=2$ and insert the elements of the the Pauli matrices now:

$$
Y^{00}-Y^{03}+Y^{30}-Y^{33}-Y^{11}+\mathrm{i} Y^{12}-\mathrm{i} Y^{21}-Y^{22}=Y_{\mu}^{\mu} .
$$

For the other three non-vanishing components of the left-hand side of equation 4.3.28 we get analogous results, so that finally we are left with the right-hand side

Plugged into eq. 4.3.27 this directly proofs eq. 4.3.26.

Finally there could be independent contractions of $\hat{\Theta}_{1(j)}, \hat{\Theta}_{1(j)}, \hat{\mathrm{X}}_{1(1)+}$ and $\hat{\mathrm{X}}_{1(2)-}$ with more spinoral matrices than one between the spinors.

For more than two matrices in a row, at least one of them appears double. If it is necessary, these can be brought next to each other using eq. A.1.9 and then one has the situation of eq. 4.3.24).

Most possibilities with two matrices can be reduced to the chosen set of invariants with eqns. 4.3.21), 4.3.22), 4.3.24). For the rest, where there are no equal indices, which allow those possibilities, one finds identities raising and lowering spinoral indices or using eq. A.1.9 and thus switching the indices with the result, that there are only three independent invariants of this kind left:

$$
\begin{gathered}
\hat{\Theta}_{1(1)} \hat{\mathrm{X}}_{1(1)+} \hat{\tilde{\mathrm{X}}}_{1(2)-} \hat{\tilde{\Theta}}_{1(2)} \hat{\tilde{\tilde{\Theta}}}_{1(1)} \hat{\bar{\Theta}}_{1(2)}, \\
\hat{\Theta}_{1(1)} \hat{\tilde{\Theta}}_{1(2)} \hat{\tilde{\tilde{\Theta}}}_{1(1)} \hat{\tilde{\mathrm{X}}}_{1(1)+} \hat{\mathrm{X}}_{1(2)-} \hat{\bar{\Theta}}_{1(2)}, \\
\hat{\Theta}_{1(1)} \hat{\mathrm{X}}_{1(1)+} \hat{\tilde{\mathrm{X}}}_{1(2)-} \hat{\tilde{\Theta}}_{1(2)} \hat{\tilde{\tilde{\Theta}}}_{1(1)} \hat{\mathrm{X}}_{1(1)+} \hat{\tilde{\mathrm{X}}}_{1(2)-} \hat{\bar{\Theta}}_{1(2)} \cdot
\end{gathered}
$$


For all of them we need to look at the equation, which we have shown in lemma 4.1. When we choose a convenient symmetric tensor and multiply the equation (4.3.28) by suitable terms, we see, that they are no new independent invariants.

We start with the invariant 4.3.31). In order to find this invariant among the terms, which eq. 4.3.28 provides, we take $Y^{\mu \nu}=\hat{X}_{1(1)+}^{\mu} \hat{X}_{1(1)+}^{\nu}$ and we multiply this equation from the right by $\hat{\tilde{\mathrm{X}}}_{1(2)-}$, so that,

$$
\hat{X}_{1(1)+}^{\mu} \hat{X}_{1(1)+}^{\nu}\left(\sigma_{\mu_{\alpha \dot{\alpha}}} \sigma_{\nu \beta \dot{\beta}}-\sigma_{\mu_{\alpha \dot{\beta}}} \sigma_{\nu \beta \dot{\alpha}}\right)\left(\hat{\tilde{\mathrm{X}}}_{1(2)-}\right)^{\dot{\alpha} \gamma}=\hat{X}_{1(1)+}^{2} \epsilon_{\alpha \beta} \bar{\epsilon}_{\dot{\alpha} \dot{\beta}}\left(\hat{\tilde{\mathrm{X}}}_{1(2)-}\right)^{\dot{\alpha} \gamma}
$$

We now multiply the spinors $\hat{\Theta}_{1(1)}, \hat{\tilde{\Theta}}_{1(2)}$ on both sides of this equation:

$$
\begin{aligned}
& \hat{\Theta}_{1(1)} \hat{\mathrm{X}}_{1(1)+} \hat{\tilde{\mathrm{X}}}_{1(2)-} \hat{\tilde{\Theta}}_{1(2)}\left(\hat{\mathrm{X}}_{1(1)+}\right)_{\beta \dot{\beta}}-\left(\hat{\Theta}_{1(1)} \hat{\mathrm{X}}_{1(1)+}\right)_{\dot{\beta}}\left(\hat{\mathrm{X}}_{1(1)+} \hat{\tilde{\mathrm{X}}}_{1(2)-} \hat{\tilde{\Theta}}_{1(2)}\right)_{\beta} \\
= & -\hat{X}_{1(1)+}^{2} \hat{\tilde{\Theta}}_{1(1)_{\beta}}\left(\hat{\Theta}_{1(2)} \hat{\mathrm{X}}_{1(2)-}\right)_{\dot{\beta}} .
\end{aligned}
$$

Finally we resort and multiply by the inverse of $\hat{\mathrm{X}}_{1(1)+}$ from the left and then by the spinors, $\hat{\tilde{\tilde{\Theta}}}_{1(1)}$ and $\hat{\bar{\Theta}}_{1(2)}$,

$$
\begin{aligned}
& \hat{\Theta}_{1(1)} \hat{\mathrm{X}}_{1(1)+} \hat{\tilde{\mathrm{X}}}_{1(2)-} \hat{\tilde{\Theta}}_{1(2)} \hat{\tilde{\tilde{\Theta}}}_{1(1)}\left(\hat{\mathrm{X}}_{1(1)+}\right)^{-1} \hat{\mathrm{X}}_{1(1)+} \hat{\bar{\Theta}}_{1(2)} \\
= & -\hat{\Theta}_{1(1)} \hat{\mathrm{X}}_{1(1)+} \hat{\bar{\Theta}}_{1(2)} \hat{\tilde{\tilde{\Theta}}}_{1(1)} \hat{\tilde{\mathrm{X}}}_{1(2)-} \hat{\tilde{\Theta}}_{1(2)}-\hat{\tilde{\tilde{\Theta}}}_{1(1)} \hat{\tilde{\mathrm{X}}}_{1(1)+} \hat{\widetilde{\Theta}}_{1(1)} \hat{\Theta}_{1(2)} \hat{\mathrm{X}}_{1(2)-} \hat{\bar{\Theta}}_{1(2)} \\
= & I_{112} I_{221}+I_{111} I_{222} .
\end{aligned}
$$

This is exactly the invariant 4.3.31). With an analogous calculation we also can express the second invariant 4.3.32). To do so one takes $Y^{\mu \nu}=\hat{X}_{1(2)-}^{\mu} \hat{X}_{1(2)-}^{\nu}$ and multiplies eq. 4.3 .28 from left by $\hat{\mathrm{X}}_{1(1)+}$. The fitting multiplications by the inverse of $\hat{\mathrm{X}}_{1(2)-}$ and the spinors then lead to the result,

$$
\hat{\Theta}_{1(1)} \hat{\tilde{\Theta}}_{1(2)} \hat{\tilde{\Theta}}_{1(1)} \hat{\tilde{X}}_{1(1)+} \hat{\mathrm{X}}_{1(2)-} \hat{\bar{\Theta}}_{1(2)}=I_{121} I_{212}+I_{111} I_{222} .
$$

For the third invariant (4.3.33) we multiply eq. 4.3 .28 by itself and contract the indices, so that one summand has - after expanding the brackets - the form of the matrices between the spinors in this invariant.

$$
\begin{aligned}
& \bar{\epsilon}^{\dot{\alpha} \dot{\delta}} \epsilon^{\beta \delta} \bar{\epsilon}^{\dot{\beta} \dot{\epsilon}} \epsilon^{\gamma \epsilon} \hat{X}_{1(1)+}^{\mu} \hat{X}_{1(1)+}^{\rho}\left(\sigma_{\mu_{\alpha \dot{\alpha}}} \sigma_{\rho_{\epsilon \dot{\epsilon}}}-\sigma_{\mu_{\alpha \dot{\epsilon}}} \sigma_{\rho_{\epsilon \dot{\alpha}}}\right) \\
& \cdot \hat{X}_{1(2)-}^{\nu} \hat{X}_{1(2)-}^{\lambda}\left(\sigma_{\nu \delta \dot{\delta}} \sigma_{\lambda \gamma \dot{\gamma}}-\sigma_{\nu \delta \dot{\gamma}} \sigma_{\lambda \gamma \dot{\delta}}\right) \\
= & \hat{X}_{1(1)+}^{2} \hat{X}_{1(2)-}^{2} \delta_{\alpha}{ }^{\beta} \delta_{\dot{\gamma}}^{\dot{\beta}} .
\end{aligned}
$$


We multiply this equation with the spinors and get

$$
\begin{aligned}
& \hat{\Theta}_{1(1)} \hat{\mathrm{X}}_{1(1)+} \hat{\tilde{\mathrm{X}}}_{1(2)-} \hat{\tilde{\Theta}}_{1(2)} \hat{\tilde{\Theta}}_{1(1)} \hat{\mathrm{X}}_{1(1)+} \hat{\tilde{\mathrm{X}}}_{1(2)-} \hat{\bar{\Theta}}_{1(2)}+\hat{\Theta}_{1(1)} \hat{\mathrm{X}}_{1(1)+} \hat{\tilde{\mathrm{X}}}_{1(2)-} \hat{\mathrm{X}}_{1(1)+} \hat{\bar{\Theta}}_{1(1)} \\
& \cdot \hat{\Theta}_{1(2)} \hat{\mathrm{X}}_{1(2)-} \hat{\hat{\Theta}}_{1(2)}-2 \hat{X}_{1(1)+} \cdot \hat{X}_{1(2)-} \hat{\Theta}_{1(1)} \hat{\mathrm{X}}_{1(1)+} \hat{\bar{\Theta}}_{1(1)} \hat{\Theta}_{1(2)} \hat{\mathrm{X}}_{1(2)-} \hat{\bar{\Theta}}_{1(2)} \\
& +\hat{\Theta}_{1(1)} \hat{\mathrm{X}}_{1(1)+} \hat{\bar{\Theta}}_{1(1)} \hat{\Theta}_{1(2)} \hat{\mathrm{X}}_{1(2)-} \hat{\tilde{\mathrm{X}}}_{1(1)+} \hat{\mathrm{X}}_{1(2)-} \hat{\bar{\Theta}}_{1(2)} \\
= & \hat{X}_{1(1)+}^{2} \hat{X}_{1(2)-}^{2} \hat{\Theta}_{1(1)} \hat{\widetilde{\Theta}}_{1(2)} \hat{\tilde{\tilde{\Theta}}}_{1(1)} \hat{\bar{\Theta}}_{1(2)} .
\end{aligned}
$$

We have already seen, that the terms with products of three matrices can be reduced to sums of only one matrix between the spinors. Hence we conclude, that the invariant (4.3.33) is equal to an expression of only the eight nilpotent invariants, which we have chosen in the beginning of this section and non-nilpotent invariants. Thus this section concludes with the corollary:

Corollary. There are no fully invariant contractions of $\hat{\tilde{\Theta}}_{1(j)}$, $\hat{\tilde{\Theta}}_{1(j)}$ and $\tilde{\mathrm{X}}_{1(i) \pm}$, which cannot be expressed by the chosen set of ten invariants.

Contractions, which do not have an equal number of $\hat{\tilde{\Theta}}_{1(j)}$ and $\hat{\tilde{\tilde{\Theta}}}_{1(j)}$, are not full, but only partial invariants, which are discussed in appendix C.4.

\subsubsection{Monomials of nilpotent invariants}

At this point we can now in principle express all scalar nilpotent invariant functions of four points of superspace in terms of the eight invariants in eqns. 4.3.3 and (4.3.4) and non-nilpotent invariants. Now we also want to choose a basis of polynomials of these nilpotent invariants, which consists of monomials of the eight nilpotent invariants. In the next section we see, that any full four-point invariant can be expanded as such a polynomial, if one allows the coefficients to be functions of non-nilpotent invariants, e.g. the superconformal invariants.

Because third powers of each of the spinors in eqns. (4.2.18) and 4.2 .19 vanish and because of some identities, which we show in this section, the number of possible linear independent monomials of the nilpotent invariants is only 36 including zeroth and first degree.

The following set $\mathfrak{I}$ is chosen and sorted by degree and consecutive numbers here:

$$
\begin{aligned}
\mathfrak{I}_{0,1}= & (1), \\
\mathfrak{I}_{1,1 \ldots 8}= & \left(I_{111}, I_{112}, I_{121}, I_{122}, I_{211}, I_{212}, I_{221}, I_{222}\right), \\
\mathfrak{I}_{2,1 \ldots 18}= & \left(I_{111}{ }^{2}, I_{112}{ }^{2}, I_{121}{ }^{2}, I_{122}{ }^{2}, I_{111} I_{112}, I_{111} I_{121}, I_{111} I_{122}, I_{112} I_{121},\right. \\
& I_{112} I_{122}, I_{121} I_{122}, I_{111} I_{221}, I_{122} I_{221}, I_{111} I_{222}, I_{122} I_{211}, I_{111} I_{212} \\
& \left.I_{122} I_{212}, I_{112} I_{221}, I_{212} I_{221}\right) \\
\mathfrak{I}_{3,1 \ldots 8}= & \left(I_{111}^{2} I_{122}, I_{111}^{2} I_{222}, I_{112}^{2} I_{121}, I_{112}^{2} I_{221}, I_{121}^{2} I_{112}, I_{121}^{2} I_{212}, I_{122}^{2} I_{111}, I_{122}^{2} I_{211}\right), \\
\mathfrak{I}_{4,1}= & I_{111}^{2} I_{122}^{2},
\end{aligned}
$$

with $i, k, j, l=1,2$. They are sorted with the first index being the degree. 
In the following the relations between the monomials, which have been left out in the choice of the subset, and the chosen ones are given using properties of the Pauli matrices and eqns. 4.3.21)- 4.3.22.

Both of the latter equations are applied for the following cases of products of two invariants containing the same spinors $\Theta_{j}$ and $\bar{\Theta}_{k}$ with $j, k=1,2$ :

$$
\begin{aligned}
I_{1 j k} I_{2 j k} & =\frac{1}{4} \hat{X}_{1(1)+}^{\mu} \hat{X}_{1(2)-}^{\nu} \operatorname{tr}\left(\sigma_{\mu} \bar{\sigma}_{\nu}\right) \hat{\Theta}_{1(j)} \hat{\tilde{\Theta}}_{1(j)} \hat{\tilde{\Theta}}_{1(k)} \hat{\bar{\Theta}}_{1(k)} \\
& =\frac{\hat{X}_{1(1)+}^{\mu} \hat{X}_{1(2)-\mu}}{\hat{X}_{1(1)+}^{2}} I_{1 j k} I_{1 j k}
\end{aligned}
$$

for $j, k=1,2$. In the last step we used eq. A.1.11 for the trace and eq. 4.3.25 to get to $I_{1 j k}$ 's.

Only either eq. 4.3.21 or eq. 4.3.22 can be used to get

$$
I_{2 j k} I_{2 m n}=\frac{\hat{X}_{1(2)-}^{2}}{\hat{X}_{1(1)+}^{2}} I_{1 j k} I_{1 m n}
$$

for $j, k, m, n=1,2$ with $j=m \vee k=n$. The first step to show this is the same calculation as for eqns. 4.3.23 and 4.3.25. $\hat{X}_{1(1)+}$ in the latter equations is just replaced by $\hat{X}_{1(2)-}$ here. In a second step we use eqns. 4.3.23 and 4.3.25) to get the right hand side of 4.3.43).

The rest of the monomials of degree 2 , which are not elements of $\mathfrak{I}_{2, i}$ and have not been expressed by them, can only be expressed in terms of two or more monomials from $\mathfrak{I}_{2, i}$. This rest devides into three different cases of monomials $I_{i j k} I_{l m n}$ :

$$
\begin{aligned}
& \text { 1. } i=1, l=2,(j \neq m \wedge k=n) \vee(j=m \wedge k \neq n), \\
& \text { 2. } i=1, l=2, j \neq m \wedge k \neq n, \\
& \text { 3. } i=l=2 j \neq m \wedge k \neq n .
\end{aligned}
$$

There are four of the first case, one of the second and one of third case, which are not elements of $\mathfrak{I}_{2, i}$.

Case 1: In this case only either 4.3.21 or 4.3.22 can be used and not both as in eq. 4.3.41. As $i \neq j$, the sigma matrices are not contracted with a symmetric tensor, as in eq. 4.3.43. But still the latter way leads to success, if we symmetrize the products in the following way:

$$
I_{1 j k} I_{2 m n}+I_{2 j k} I_{1 m n}
$$

Here we continue the calcuation now for $j=m \wedge k \neq n$ and keep in mind, that for $j \neq m \wedge k=n$ things work analoguesly. So we have:

$$
\begin{aligned}
I_{1 j k} I_{2 j n}+I_{2 j k} I_{1 j n} & =\left(\hat{X}_{1(1)+}^{\mu} \hat{X}_{1(2)-}^{\nu}+\hat{X}_{1(2)-}^{\mu} \hat{X}_{1(1)+}^{\nu}\right) \hat{\Theta}_{1(j)} \sigma_{\mu} \hat{\bar{\Theta}}_{1(k)} \hat{\Theta}_{1(j)} \sigma_{\nu} \hat{\bar{\Theta}}_{1(n)} \\
& =2 \frac{\hat{X}_{1(1)+}^{\mu} \hat{X}_{1(2)-\mu}}{\hat{X}_{1(1)+}^{2}} I_{1 j k} I_{1 j n} .
\end{aligned}
$$


Due to the symmetrization the situation is the same as in eq. 4.3.43). The factor two comes from the contraction of the tensor in the brackets.

This calculation actually shows us the cause, why $I_{111} I_{212}, I_{111} I_{221}, I_{212} I_{122}$ and $I_{221} I_{122}$ are elements of the set $\mathfrak{I}$. They are needed to express the four monomials $I_{211} I_{112}, I_{211} I_{121}, I_{112} I_{222}$ and $I_{121} I_{222}$ :

$$
\begin{aligned}
& I_{211} I_{112}=2 \hat{X}_{1(1)+}^{\mu} \hat{X}_{1(2)-\mu} I_{111} I_{112}-I_{111} I_{212}, \\
& I_{211} I_{121}=2 \hat{X}_{1(1)+}^{\mu} \hat{X}_{1(2)-\mu} I_{111} I_{121}-I_{111} I_{221}, \\
& I_{112} I_{222}=2 \hat{X}_{1(1)+}^{\mu} \hat{X}_{1(2)-\mu} I_{112} I_{122}-I_{212} I_{122}, \\
& I_{121} I_{222}=2 \hat{X}_{1(1)+}^{\mu} \hat{X}_{1(2)-\mu} I_{121} I_{122}-I_{221} I_{122} .
\end{aligned}
$$

This is one of the choices, that could be taken freely. Any of the monomials on the left hand side could have been chosen as an element of $\mathfrak{I}$ instead of the element at the very right of the equations.

Case 2: This case consists of the monomial $I_{121} I_{212}$. The symmetrization of the respective first indices is not enough here because we face the same problem as in eq. 4.3.26), that neither 4.3.21 nor 4.3.22 can be applied. So we need more monomials to express $I_{121} I_{212}$, so that we can apply lemma 4.1 also here:

$$
\begin{aligned}
& I_{121} I_{212}+I_{112} I_{221}+I_{111} I_{222}+I_{122} I_{211} \\
= & \left(\hat{X}_{1(1)+}^{\mu} \hat{X}_{1(2)-}^{\nu}+\hat{X}_{1(2)-}^{\mu} \hat{X}_{1(1)+}^{\nu}\right) \hat{\Theta}_{1(1)}^{\alpha} \hat{\bar{\Theta}}_{1(1)}^{\dot{\alpha}} \hat{\Theta}_{1(2)}^{\beta} \hat{\bar{\Theta}}_{1(2)}^{\dot{\beta}}\left(\sigma_{\mu_{\alpha \dot{\alpha}}} \sigma_{\nu \beta \dot{\beta}}-\sigma_{\mu_{\alpha \dot{\beta}}} \sigma_{\nu \beta \dot{\alpha}}\right) \\
= & 2 \frac{\hat{X}_{1(1)+}^{\mu} \hat{X}_{1(2)-\mu}}{\hat{X}_{1(1)+}^{2}}\left(I_{111} I_{122}+I_{112} I_{121}\right) .
\end{aligned}
$$

In the last line we get twice the same contraction of four-vectors and we use eq. 4.3.26 to get back to the $I_{1 j k}$ 's. Also this illuminates the choice of $\mathfrak{I}$. $I_{121} I_{212}$ could replace any of the three other monomials in the first line of this equation.

Case 3: At last the monomial $I_{211} I_{222}$ is just $I_{111} I_{122}$ with $\hat{X}_{1(1)+}^{\mu}$ replaced by $\hat{X}_{1(2)-}^{\mu}$. So the calculation is nothing but 4.3.26), which is once applied in one direction with the replacement just mentioned and then applied "backwards" in its original form. The result is:

$$
I_{211} I_{222}+I_{212} I_{221}=\frac{\hat{X}_{1(2)-}^{2}}{\hat{X}_{1(1)+}^{2}}\left(I_{111} I_{122}+I_{112} I_{121}\right) .
$$

The choice of $I_{212} I_{221}$ within $\mathfrak{I}$ is, of course, free as well.

Now we have come to an end of the discussion of monomials of degree 2, many of the identities given above can be used to relate monomials of degree 3. However, there are still products of three different nilpotent invariants like $I_{111} I_{112} I_{121}$, which are neither in our selection nor can be reduced directly to the chosen monomials with the equations we have had. But obviously there are pairs of spinoral functions 
of three points in these monomials, for which eqns. 4.3.21 and 4.3.21 can be applied.

First of all we see, that all monomials, which we need to look at here, are of the form $I_{i j k} I_{l j o} I_{m n o}$ for two reasons. First, as there are only two possible values for each second index, two of them have to be the same. The same is true for the third indices. Second, if both the second and the third indices are the same in the same two invariants in the monomial, one can apply either eq. 4.3.41) or eq. 4.3.43 to those two and gets a monomial in $\mathfrak{I}$.

Now we apply eqns. 4.3.21 and 4.3.22 to $I_{i j k} I_{l j o} I_{m n o}$ :

$$
\begin{aligned}
I_{i j k} I_{l j o} I_{m n o} & =\hat{X}_{1(i)^{+} /-}^{\mu} \hat{X}_{1(l)+/}^{\nu} \hat{X}_{1(m)^{+} /-}^{\rho} \hat{\Theta}_{1(j)} \sigma_{\mu} \hat{\bar{\Theta}}_{1(k)} \hat{\Theta}_{1(j)} \sigma_{\nu} \hat{\bar{\Theta}}_{1(o)} \hat{\Theta}_{1(n)} \sigma_{\rho} \hat{\bar{\Theta}}_{1(o)} \\
& =-\frac{1}{4} \hat{\Theta}_{1(j)} \hat{\tilde{\Theta}}_{1(j)} \hat{\tilde{\Theta}}_{1(o)} \hat{\Theta}_{1(o)} \hat{X}_{1(i)^{+/} /}^{\mu} \hat{X}_{1(l)^{+} /-}^{\nu} \hat{X}_{1(m)^{+} /-}^{\rho} \hat{\Theta}_{1(n)} \sigma_{\rho} \bar{\sigma}_{\nu} \sigma_{\mu} \hat{\bar{\Theta}}_{1(k)},
\end{aligned}
$$

where $1(\cdot)^{+} /-$is either $1(1)+$ or $1(2)-$. At this point there are two distinct cases: Either $l=m \vee l=i$ or $i=m \neq l$.

In the first case there is a product of two Pauli matrices contracted with a symmetric tensor, so that we can use eq. 4.3.24). At last we apply eq. 4.3.25 and get

$$
\begin{aligned}
I_{l j k} I_{l j o} I_{m n o} & =-\frac{1}{2} \frac{\hat{X}_{1(l)+/}^{2}}{\hat{X}_{1(1)+}^{2}} I_{1 j o}{ }^{2} I_{m n k} \\
I_{i j k} I_{l j o} I_{l n o} & =-\frac{1}{2} \frac{\hat{X}_{1(l)+/}^{2}}{\hat{X}_{1(1)+}^{2}} I_{1 j o}{ }^{2} I_{i n k}
\end{aligned}
$$

For the second case two Pauli matrices have to be interchanged with the help of A.1.9, so that we can procede as in the first case. We can also already insert eq. 4.3.25) and have

$$
\begin{aligned}
I_{i j k} I_{l j o} I_{\text {ino }} & =-\frac{1}{2} \frac{1}{\hat{X}_{1(1)+}^{2}} I_{1 j o}{ }^{2} \hat{X}_{1(i)^{+} /-}^{\mu} \hat{X}_{1(l)^{+} /-}^{\nu} \hat{X}_{1(i)^{+} /-}^{\rho} \hat{\Theta}_{1(n)}\left(2 \eta_{\nu \rho}-\sigma_{\nu} \bar{\sigma}_{\rho}\right) \sigma_{\mu} \hat{\bar{\Theta}}_{1(k)} \\
& =-\frac{\hat{X}_{1(1)+}^{\nu} \hat{X}_{1(2)-\nu}}{\hat{X}_{1(1)+}^{2}} I_{1 j o}{ }^{2} I_{i n k}+\frac{1}{2} \frac{\hat{X}_{1(i)+/}^{2}}{\hat{X}_{1(1)+}^{2}} I_{1 j o}{ }^{2} I_{l n k} .
\end{aligned}
$$

Finally we are left with only one monomial of degree 4 , which we need to relate to the one from $\mathfrak{I}$, so that all other can be given by the latter one using also the equations, which we have calculated for the monomials of lower degree. This missing link is $I_{112}{ }^{2} I_{121}{ }^{2}$. But one can easily see, that it is equal to $I_{111}^{2} I_{122}^{2}$. Eq. 4.3.26 has to be plugged in only twice

$$
I_{112}^{2} I_{121}^{2}=\hat{X}_{1(1)+} \hat{\Theta}_{1(1)} \hat{\tilde{\Theta}}_{1(1)} \hat{\tilde{\tilde{\Theta}}}_{1(2)} \hat{\bar{\Theta}}_{1(2)} \hat{X}_{1(1)+} \hat{\Theta}_{1(2)} \hat{\tilde{\Theta}}_{1(2)} \hat{\tilde{\tilde{\Theta}}}_{1(1)} \hat{\bar{\Theta}}_{1(1)}
$$

We can sort this expression differently, which directly leads us to the desired monomial $\mathfrak{I}_{4,1}$ :

$$
I_{112}{ }^{2} I_{121}{ }^{2}=\hat{X}_{1(1)+} \hat{\Theta}_{1(1)} \hat{\widetilde{\Theta}}_{1(1)} \hat{\tilde{\tilde{\Theta}}}_{1(1)} \hat{\bar{\Theta}}_{1(1)} \hat{X}_{1(1)+} \hat{\Theta}_{1(2)} \hat{\widetilde{\Theta}}_{1(2)} \hat{\tilde{\Theta}}_{1(2)} \hat{\bar{\Theta}}_{1(2)}=I_{111}^{2} I_{122}^{2} .
$$


The following corrolary results from the discussion of products of nilpotent invariants in this section and the construction of the four-point invariants in the preceding sections.

Corollary. Any full four-point invariant can be expressed as a linear combination of the monomials $\mathfrak{I}_{i j}$ with coefficients, which are functions of two fixed independent non-nilpotent invariants.

In the next section we finalize this notion by the proof, that this forms a unique expansion.

\subsubsection{Expansion of invariant functions in nilpotent invariants}

The transformation properties of superconformal variables leave no room for superconformal invariants, which cannot be expressed by those, which we constructed above. Any invariant function can be given in terms of a finite expansion into nilpotent invariants with coefficients, which are functions of non-nilpotent invariants. In the following theorem we give a unique expension of this kind with the help of the set $\mathfrak{I}$.

Theorem 4.1. Let $f$ be an arbitrary invariant function of four points of superspace. Then it can be expanded as

$$
f\left(\mathcal{I}_{1}, \mathcal{I}_{2}, I_{i j k}\right)=\sum_{p=1}^{4} \sum_{q=1}^{n_{p}} f_{p, q}\left(\mathcal{I}_{1}, \mathcal{I}_{2}\right) \mathfrak{I}_{p, q}
$$

and the coefficient functions $f_{p, q}\left(\mathcal{I}_{1}, \mathcal{I}_{2}\right)$ are uniquely determined.

From the last sections we know, that the set $\mathfrak{I}$ is a sufficiently large set because all monomials of $I_{i j k}$ can be expressed by those in this set and the superconformal cross ratios $\mathcal{I}_{1}$ and $\mathcal{I}_{2}$. This leaves the uniqueness to be shown. For this we have to show, that there are no dependencies between the invariants in the set $\mathfrak{I}$. This task is divided in this section into the smaller questions, if the monomials of each degree are independent from each other. For degree one we present first a method, which focuses on the lowest order of these invariants. Afterward we show the same differently within a lemma and two other lemmas will provide the second and third degree. At the end we show the theorem by essentially combining the lemmas.

We now show, that all eight nilpotent invariants are independent, meaning, that non of them can be expressed by the others and non-nilpotent invariants. In order to do so, we look at the lowest order of these invariants given in appendix C.1.

$I_{111}$ and $I_{222}$ are especially simple, also in lowest order. Here we use the comment "l.o." above the equals sign to express the equality to lowest order in Graßmann variables:

$$
I_{111} \stackrel{\text { l.o. }}{=}-\theta_{12} \tilde{\mathrm{x}}_{12}^{-1} \bar{\theta}_{12}-\theta_{24} \tilde{\mathrm{x}}_{24}^{-1} \bar{\theta}_{24}+\theta_{14} \tilde{\mathrm{x}}_{14}^{-1} \bar{\theta}_{14} .
$$

The latter can be derived from this - just as any $I_{2 j k}$ can be derived from one $I_{1 \mathrm{~nm}}$ - with a look on eqns. (4.3.3) and (4.3.4). The normalizations in the denominators 
are the same, which is in lowest order $x_{24}{ }^{2} /\left(x_{21}{ }^{2} x_{14}{ }^{2}\right)$. Indices in the numerator differ between $I_{111}$ and $I_{222}$. 1 is replaced by 2 and so with the three point functions (eqns. 4.2.13), 4.2.18) and 4.2.19) inserted, 2 is replaced by 3 therein. So we multiply eq. (4.3.58) by the normalization, replace each index 2 by 3 and divide by the normalization again to get

$$
I_{222} \stackrel{\text { 1.o. }}{=} \frac{x_{12}^{2} x_{43}^{2}}{x_{13}^{2} x_{42}{ }^{2}}\left(-\theta_{13} \tilde{\mathrm{x}}_{13}^{-1} \bar{\theta}_{13}-\theta_{34} \tilde{\mathrm{x}}_{34}^{-1} \bar{\theta}_{34}+\theta_{14} \tilde{\mathrm{x}}_{14}^{-1} \bar{\theta}_{14}\right) \text {. }
$$

The key to show the independence of the invariants are terms, which appear in the other six $I_{i j k}$. They contain terms with a product of three Pauli matrices, which cannot be simplified in the same way as in $I_{i i i}$. E.g. in the lowest order of $I_{112}$, as it is given in eq. C.1.6), we find $\theta_{13} \tilde{\mathrm{x}}_{13}^{-1} \tilde{\mathrm{x}}_{12} \tilde{\mathrm{x}}_{24}^{-1} \bar{\theta}_{24}$. With the help of the relations between different intervals the indices of $\tilde{x}_{i j}$ can be changed with the cost of other terms of the form $\theta_{31} \tilde{x}_{k l} \bar{\theta}_{42}$ mostly with some factor of intervals squares, but a term with three Pauli matrices always survives.

For the Graßmannian intervals, $\theta_{i j}$ and $\bar{\theta}_{i j}$, there is in each case one unique way, in which terms with three sigma matrices can be simplified, so that maximally two of these terms survive, which cannot be united.

We now look at all these terms ignoring the indices of the intervals, $\mathrm{x}_{i j}$, because they can be arranged to be the same in all invariants. We leave out all other terms or prefactors:

$$
\begin{array}{ll}
I_{112}: & \theta_{31} \operatorname{xxx} \bar{\theta}_{41}-\theta_{31} \operatorname{xxx} \bar{\theta}_{21}, \\
I_{121}: & \theta_{21} \operatorname{xxx} \bar{\theta}_{31}-\theta_{41} \operatorname{xxx} \bar{\theta}_{31}, \\
I_{122}: & \theta_{31} \mathrm{xxx} \bar{\theta}_{41}-\theta_{41} \mathrm{xxx} \bar{\theta}_{31}, \\
I_{211}: & \theta_{41} \operatorname{xxx} \bar{\theta}_{21}-\theta_{21} \mathrm{xxx} \bar{\theta}_{41}, \\
I_{212}: & \theta_{41} \mathrm{xxx} \bar{\theta}_{21}-\theta_{31} \mathrm{xxx} \bar{\theta}_{21}, \\
I_{221}: & \theta_{21} \mathrm{xxx} \bar{\theta}_{31}-\theta_{21} \mathrm{xxx} \bar{\theta}_{41} .
\end{array}
$$

These terms would have to match to give a relation between these invariants. Also no factor of a non-nilpotent invariant could change this because in lowest order these are just combinations of conformal cross ratios. So in order to get a vanishing combination containing these six invariants necessarily the Grassmannian intervals would have to cancel in the summands above.

One can start at any invariant and finds, that there is only one way to achieve this. At the end this one combination does not vanish overall. For example, we can start with $I_{112}$. Its first summand is also in $I_{122}$. The difference of these two invariants contains:

$$
I_{112}-I_{122}: \quad \theta_{41} \mathrm{xxx} \bar{\theta}_{31}-\theta_{31} \mathrm{xxx} \bar{\theta}_{21} .
$$

We continue in this way always adding or subtracting the invariant, which cancels one of the summands. This way we have to go through all six invariant to finally end up with the total cancellation of the terms with three Pauli-matrices:

$$
I_{112}-I_{122}+I_{211}-I_{212}+I_{121}-I_{221}: \quad 0 .
$$


As this algorithm goes through all terms in eqns. 4.3.60)- 4.3.65), which all appear twice, it is indifferent, at which term it is started or which route it takes. Thus this is the only possible combination.

The question to pose at this point is, if this combination is independent from the two nilpotent invariants we looked at before, $I_{111}$ and $I_{222}$. Starting from another perspective answers this question.

As already stated above, $I_{111}$ and $I_{222}$ times their normalization are essentially the same functions, but once with variables $z_{1} z_{2}$ and $z_{4}$ and once with $z_{1}, z_{3}$ and $z_{4}$. But we have seen already a third possibility in the construction of the three point invariant. $I_{3 P F}$ (cf. eq. 4.1.14) has the same function with $z_{1} z_{2}$ and $z_{3}$ in the numerator with a different denominator and a factor of $-4 \mathrm{i}$ :

$$
\begin{aligned}
I_{3 P F} & =-4 i \hat{\Theta}_{1} \hat{\mathrm{X}}_{1+} \hat{\bar{\Theta}}_{1} \frac{1}{\hat{X}_{1+}^{2}} \\
& =-4 \mathrm{i} I_{0} \frac{1}{\hat{X}_{1+}^{2}} .
\end{aligned}
$$

In the last step we define the invariant $I_{0} \square$, which has the same normalization as $I_{111}$ and $I_{222}$.

At this point we need only to plug eqns. 4.2.25)-4.2.27) into $I_{0}$ and find the combination in eq. 4.3.67) in it:

$$
\begin{aligned}
I_{0}= & I_{111}-I_{112}-I_{121}+I_{122}-I_{211}+I_{212}+I_{221}-I_{222} \\
& -4 \mathrm{i}\left(2 I_{112}^{2}-2 I_{111} I_{112}-2 I_{112} I_{122}+I_{111} I_{122}+I_{112} I_{121}\right) \\
& +4 I_{111}^{2} I_{222}+8 I_{112}^{2} I_{121} .
\end{aligned}
$$

The last step in establishing the independence of the eight $I_{i j k}$ hence is to show the independence of $I_{111}, I_{222}$ and $I_{0}$.

We have seen in eq. 4.3.68), that the normalization can be changed with the help of cross ratios. These non-nilpotent invariants are irrelevant to our discussion of the independence, as we defined it above. So we look at the lowest order of $I_{111}$ with $z_{2}$ replaced by $z_{3}$ instead of $I_{222}$, which means nothing else, but dropping the cross ratio in eq. 4.3.59

$$
\frac{x_{13}^{2} x_{42}^{2}}{x_{12}{ }^{2} x_{43}{ }^{2}}\left(I_{222}\right)_{\text {l.o. }}=-\theta_{13} \tilde{\mathrm{x}}_{13}^{-1} \bar{\theta}_{13}-\theta_{34} \tilde{\mathrm{x}}_{34}^{-1} \bar{\theta}_{34}+\theta_{14} \tilde{\mathrm{x}}_{14}^{-1} \bar{\theta}_{14} .
$$

For $I_{0}$ we get similarly:

$$
\frac{x_{13}^{2} x_{24}^{2}}{x_{14}{ }^{2} x_{23}{ }^{2}}\left(I_{0}\right)_{\text {l.o. }}=-\theta_{12} \tilde{\mathrm{x}}_{12}^{-1} \bar{\theta}_{12}-\theta_{23} \tilde{\mathrm{x}}_{23}^{-1} \bar{\theta}_{23}+\theta_{13} \tilde{\mathrm{x}}_{13}^{-1} \bar{\theta}_{13}
$$

One could now expand the terms in such a way, that only $\theta_{1 i}, \tilde{\mathrm{x}}_{1 i}^{-1}$ and $\bar{\theta}_{1 i}$ were left to get down to a set of variables, which are unsubstitutable among themselves. But we actually already have such a set with the terms $\theta_{i j} \tilde{\mathrm{x}}_{i j}^{-1} \bar{\theta}_{i j}$ as one entity. If we

\footnotetext{
${ }^{1}$ This invariant is the negative of $I_{\Sigma}$ in $\mathrm{Knu11}$
} 
would substitute the individual intervals by sums of other intervals, mixed terms would appear, which could not be taken together in any other way than back to the form we started from. As we see that each of the three invariants contains one $\theta_{i j} \tilde{\mathrm{x}}_{i j}^{-1} \bar{\theta}_{i j}$, which the other two do not, hey have to be independent.

There is another possibility to show, that the eight invariants $I_{i j k}$ are independent, but it does not give the insight into the lowest order structure of these invariants, which we will also use in the discussion of the four-point function. Nonetheless we go through these calculations now as well because it is also the way to show the independence of the higher order monomials.

We want to show the following:

Lemma 4.2. Let $\alpha_{i j k}\left(\mathcal{I}_{1}, \mathcal{I}_{2}\right)$ be functions of non-nilpotent invariants, so that

$$
\sum_{i, j, k=1}^{2} \alpha_{i j k}\left(\mathcal{I}_{1}, \mathcal{I}_{2}\right) I_{i j k}=0
$$

then

$$
\forall i, j, k \in\{1,2\}: \alpha_{i j k}=0 .
$$

Proof. If we multiply eq. 4.3.73 by $I_{122}{ }^{2} I_{111}$, only two summands survive because of the nilpotency:

$$
0=\alpha_{111} I_{111}^{2} I_{122}^{2}+\alpha_{211} I_{111} I_{211} I_{122}^{2}=\left(\alpha_{111}+\alpha_{211} A\right) I_{111}^{2} I_{122}^{2},
$$

where eq. 4.3.41 is used with the abbreviation $A=\frac{\hat{X}_{1(1)+}^{\mu} \hat{X}_{1(2)-\mu}}{\hat{X}_{1(1)+}^{2}}$. These two summands are also left, if eq. 4.3.73) is multiplied by $I_{122}{ }^{2} I_{211}$ :

$$
0=\alpha_{111} I_{111} I_{211} I_{122}{ }^{2}+\alpha_{211} I_{211}^{2} I_{122}{ }^{2}=\left(\alpha_{111} A+\alpha_{211} B\right) I_{111}^{2} I_{122}{ }^{2} .
$$

This time eq. (4.3.41) is used in the first summand and eq. 4.3.43 in the other one with $B=\frac{X_{1(2)-}^{2}}{\hat{X}_{1(1)+}^{2}}$. We substract now eq. 4.3.75 times $A$ from eq. 4.3.76), so that the first summands cancel:

$$
\alpha_{211}\left(B-A^{2}\right) I_{111}^{2} I_{122}^{2}=0 .
$$

$I_{111}{ }^{2} I_{122}{ }^{2}$ and the bracket are not zero, so that we conclude $\alpha_{211}=0$ and plugged in above also $\alpha_{111}=0$.

This can be repeated with the other three pairs of coefficients, $\alpha_{112}$ and $\alpha_{212}$, $\alpha_{121}$ and $\alpha_{221}$ and $\alpha_{122}$ and $\alpha_{222}$. For each of these three pairs eq. 4.3.73 must be multiplied with two monomials of third degree analoguesly to the above calculations. These monomials are - in the same order as the pairs coefficients above $-I_{121}{ }^{2} I_{112}$ and $I_{121}{ }^{2} I_{212}, I_{112}{ }^{2} I_{121}$ and $I_{112}{ }^{2} I_{221}$ and $I_{111}{ }^{2} I_{122}$ and $I_{111}{ }^{2} I_{222}$.

This gives the result, that all coefficients have to be identically zero.

Now we want to proof the independence of the monomials of higher degree with this method. If we look at the monomials of third degree, we notice, that they are just those, with which we multiply eq. 4.3.73 in the last proof. This makes the proof of their independence stated by the next lemma especially simple. 
Lemma 4.3. Let $\alpha_{l}\left(\mathcal{I}_{1}, \mathcal{I}_{2}\right)$ with $i=1, \ldots, 8$ be functions of non-nilpotent invariants, so that

$$
\sum_{l=1}^{8} \alpha_{l}\left(\mathcal{I}_{1}, \mathcal{I}_{2}\right) \mathfrak{I}_{3, l}=0
$$

then

$$
\forall l \in\{1, \ldots 8\}: \alpha_{l}=0 .
$$

Proof. As for lemma 4.2, eq. 4.3.78 has to be multiplied by the appropriate terms, so that we get systems of equations of two coefficients, $\alpha_{l}$, each. We have the following factors and the corresponding pairs of coefficients:

$$
\begin{array}{ll}
I_{122}, I_{222}: & \alpha_{1}, \alpha_{2} ; \\
I_{121}, I_{221}: & \alpha_{3}, \alpha_{4} ; \\
I_{112}, I_{212}: & \alpha_{5}, \alpha_{6} ; \\
I_{111}, I_{211}: & \alpha_{7}, \alpha_{8} .
\end{array}
$$

The systems of equations are exactly the same as in the last proof and so also lead to the desired result here.

Finally we are left with the monomials of second degree, which require some steps of calculation more because the eighteen coefficients do not pair up so nicely.

Lemma 4.4. Let $\alpha_{l}\left(\mathcal{I}_{1}, \mathcal{I}_{2}\right)$ with $i=1, \ldots, 18$ be functions of non-nilpotent invariants, so that

$$
\sum_{l=1}^{18} \alpha_{l}\left(\mathcal{I}_{1}, \mathcal{I}_{2}\right) \mathfrak{I}_{2, l}=0
$$

then

$$
\forall l \in\{1, \ldots 18\}: \alpha_{l}=0 .
$$

Proof. The first four coefficients can be singled out by the appropriate factor. If eq. (4.3.84) is multiplied by $I_{122}^{2}, I_{121}^{2}, I_{112}^{2}$ or $I_{111}^{2}$, the terms with the coefficients $\alpha_{1}$ till $\alpha_{4}$, respectively, survive. Hence these four coefficients have to be zero.

Also here we find four pairs as in the other two cases. The following factors segregate the respective pairs of coefficients:

$$
\begin{array}{ll}
I_{121} I_{122}, I_{221} I_{122}: & \alpha_{5}, \alpha_{15} ; \\
I_{112} I_{122}, I_{212} I_{122}: & \alpha_{6}, \alpha_{13} ; \\
I_{121} I_{111}, I_{221} I_{111}: & \alpha_{9}, \alpha_{16} ; \\
I_{112} I_{111}, I_{212} I_{111}: & \alpha_{10}, \alpha_{12} .
\end{array}
$$

Here we treat the first line and then see, how the others analoguosly follow. The following two equations form after the multiplication of eq. (4.3.84) with the mentioned 
factors:

$$
\begin{aligned}
\alpha_{5} I_{111} I_{112} I_{121} I_{122}+\alpha_{15} I_{111} I_{212} I_{121} I_{122} & =-\frac{1}{2}\left(\alpha_{5}+\alpha_{15} A\right) I_{111}^{2} I_{122}^{2} \\
& =0, \\
\alpha_{5} I_{111} I_{112} I_{221} I_{122}+\alpha_{15} I_{111} I_{212} I_{221} I_{122} & =-\frac{1}{2}\left(\alpha_{5} A+\alpha_{15}\left(2 A^{2}-B\right)\right) I_{111}^{2} I_{122}^{2} \\
& =0 .
\end{aligned}
$$

$A$ and $B$ are the same as in eqns. 4.3.75 and 4.3.76. For the respective first equal signs the identities between monomials, which appear and are not in $\mathfrak{I}$, and those within this set are used (cf. eqns. 4.3.52, 4.3.54) and 4.3.56). If the first equation is multiplied by $A$ and subtracted from the second, this results in a familiar equation:

$$
-\frac{1}{2} \alpha_{15}\left(A^{2}-B\right) I_{111}^{2} I_{122}^{2}=0
$$

So the two coefficients are zero.

For the other pairs again eqns. 4.3.52, 4.3.54 and 4.3.56 are all, that is needed to show, that the resulting system of equations is not different from the one above. At this point all but six coefficients have been shown to be zero. There are no appropriate factors, with which we could separate these six coefficients into smaller groups. So we deal with a system of six equations with six unknowns. This system results from the multiplications with exactly the six monomials, to which the six coefficients belong. There is no problem, that we have not proven their independence yet. If they would depend on each other, the system of equations would be under-determined, which would show in the course of solving it.

The following equations, which are already simplified, result from the multiplication of eq. 4.3.84 by $I_{111} I_{122}, I_{112} I_{121}, I_{111} I_{222}, I_{211} I_{122}, I_{112} I_{221}$ and $I_{212} I_{221}$ :

$$
\begin{gathered}
\left(\alpha_{7}-\frac{1}{2} \alpha_{8}+\alpha_{13} A+\alpha_{14} A-\frac{1}{2} \alpha_{17} A-\alpha_{18}\left(A^{2}-\frac{1}{2} B\right)\right) I_{111}^{2} I_{122}^{2}=0 \\
\left(-\frac{1}{2} \alpha_{7}+\alpha_{8}-\frac{1}{2} \alpha_{13} A-\frac{1}{2} \alpha_{14} A+\alpha_{17} A+\alpha_{18} A^{2}\right) I_{111}^{2} I_{122}^{2}=0 \\
\left(\alpha_{7} A-\frac{1}{2} \alpha_{8} A+\alpha_{13} B+\alpha_{14} A^{2}-\frac{1}{2} \alpha_{17} B-\frac{1}{2} \alpha_{18} A B\right) I_{111}^{2} I_{122}^{2}=0 \\
\left(\alpha_{7} A-\frac{1}{2} \alpha_{8} A+\alpha_{13} A^{2}+\alpha_{14} B-\frac{1}{2} \alpha_{17} B-\frac{1}{2} \alpha_{18} A B\right) I_{111}^{2} I_{122}^{2}=0 \\
\left(-\frac{1}{2} \alpha_{7} A+\alpha_{8} A-\frac{1}{2} \alpha_{13} B-\frac{1}{2} \alpha_{14} B+\alpha_{17} B+\alpha_{18} A B\right) I_{111}^{2} I_{122}^{2}=0 \\
\left(-\alpha_{7}\left(A^{2}-\frac{1}{2} B\right)+\alpha_{8} A^{2}-\frac{1}{2} \alpha_{13} A B-\frac{1}{2} \alpha_{14} A B+\right. \\
\left.+\alpha_{17} A B+\alpha_{18} B^{2}\right) I_{111}^{2} I_{122}^{2}=0
\end{gathered}
$$

The subtraction of eq. 4.3.96 from 4.3.95 leaves only $\alpha_{13}$ and $\alpha_{14}$ :

$$
\alpha_{13}\left(B-A^{2}\right)+\alpha_{14}\left(A^{2}-B\right)=0 \Rightarrow \alpha_{14}=\alpha_{13} .
$$


Once more we used the fact, that $B-A^{2}$ is not zero. In the next step we subtract on the one hand eq. 4.3.93 times $A$ from eq. 4.3.95 and on the other hand eq. (4.3.94) times $A$ from eq. (4.3.97):

$$
\begin{aligned}
\alpha_{13}\left(B-A^{2}\right)-\frac{1}{2} \alpha_{17}\left(B-A^{2}\right)-\alpha_{18}\left(B A-A^{3}\right) & =0 \\
-\frac{1}{2} \alpha_{13}\left(B-A^{2}\right)-\frac{1}{2} \alpha_{14}\left(B-A^{2}\right)+\alpha_{17}\left(B-A^{2}\right)+\alpha_{18}\left(B A-A^{3}\right) & =0
\end{aligned}
$$

With $\alpha_{14}$ plugged in, the sum of these two equations determines $\alpha_{17}$ to be zero and - with this result and one of these equations $-\alpha_{13}=A \alpha_{18}$. Now these results can also be used to simplify eq. 4.3.94:

$$
-\frac{1}{2} \alpha_{7}+\alpha_{8}=0
$$

Eq. 4.3.95 then is

$$
\frac{3}{4} \alpha_{7} A=-\alpha_{13}\left(\frac{1}{2} B+A^{2}\right)
$$

Yet unused we finally multiply eq. (4.3.98) by $A$ and plug all previous results in, so that only $\alpha_{13}$ is left in it. The multiplication would not be needed, because $A$ is invertable. So it is only for convenience, so that we end up with

$$
\alpha_{13} \frac{2}{3}\left(B-A^{2}\right)=0
$$

Thus $\alpha_{13}$ has to vanish and all other coefficients with it except $\alpha_{17}$, which we already found out to be zero.

With these three lemmas not much more is needed to prove the theorem.

Proof of theorem 4.1: From the calculations in the proofs of the lemmas one can see, that the vanishing of the coefficients already follows, if we start from the assumption, that the linear combination only vanishes in lowest non-vanishing order of the invariants. For $i=1,2,3$ we have

$$
\sum_{l=1}^{n_{l}} \alpha_{l}\left(\mathcal{I}_{1}, \mathcal{I}_{2}\right) \mathfrak{I}_{i, l} \stackrel{\text { l.o. }}{=} 0 \Rightarrow \alpha_{l}\left(\mathcal{I}_{1}, \mathcal{I}_{2}\right)=0
$$

The reason is the non-nilpotency of the difference $\left(B-A^{2}\right)$. This makes it also impossible, that a combination of monomials of degree $i$ is equal to one or a combination of monomials of a higher degree $j>i$.

This establishes the independence of all monomials in $\mathfrak{I}$ and shows the uniqueness of the expansion 4.3.57). 


\subsubsection{Algorithmic calculations with explicit expansions of cross ra- tios and other functions of superspace coordinates}

In eq. 4.3.57) we took the invariants $\mathcal{I}_{1}$ and $\mathcal{I}_{2}$ as arguments of the coefficient functions. But every other pair of non-nilpotent invariants can be used, if it is not possible to express one invariant of this pair by the other one and nilpotent invariants. In this section we see, how we can take advantage of the nilpotency of the monomials from section 4.3 .2 and apply an algorithm, which allows us to replace the non-nilpotent invariants in the expansions of the kind of theorem 4.1 by other non-nilpotent invariants. The discussion here is completed by the Maple procedures in appendix $\mathrm{D}$, which handle these expansions.

For the computation of expansions in this paragraph we have to start with the arguments $X_{1(2)-}{ }^{2}$ and $X_{1(1)+\mu} X_{1(2)-}{ }^{\mu}$ because we encounter products of nilpotent invariants, which have to be brought into the correct form for the expansion with the help of the equations gathered in section 4.3.2. As we have not expressed these two arguments by another pair of nilpotent invariants, yet, we inevitably get these arguments in the coefficient functions. But later in this paragraph we reach this point and then are able to transfer the result to coefficient functions of cross ratios.

For these calculations it is also needed to express $\hat{X}_{1(1)+}^{2}$ in terms of the nilpotent invariant $I_{111}$. This can be done in an iterative way, which already stops after the second step here, but is a prototype to other calculations later on, especially when one wants to replace one pair of non-nilpotent invariants by another. The key lies in an equation, in which the studied invariant - here actually $\hat{X}_{1(1)-}^{2}$ - appears once with no or a non-nilpotent factor and another time multiplied with a nilpotent invariant.

We begin with the three point functions $\hat{X}_{1(1)+}^{2}$ and $\hat{X}_{1(1)-}^{2}$. These are particularly simple because the ratio cancels down, if we plug the normalization into $\hat{X}_{1(1)+}^{2}$ and $\hat{X}_{1(1)-}^{2}$,

$$
\begin{aligned}
& \hat{X}_{1(1)+}^{2}=\frac{X_{1(1)+}{ }^{2}}{\sqrt{X_{1(1)+}{ }^{2} X_{1(1)-}{ }^{2}}}=\sqrt{\frac{X_{1(1)+}{ }^{2}}{X_{1(1)-}{ }^{2}}}=\sqrt{\frac{\hat{X}_{1(1)+}^{2}}{\hat{X}_{1(1)-}^{2}}}, \\
& \hat{X}_{1(1)-}^{2}=\frac{X_{1(1)-}{ }^{2}}{\sqrt{X_{1(1)+}{ }^{2} X_{1(1)-}{ }^{2}}}=\sqrt{\frac{X_{1(1)-}{ }^{2}}{X_{1(1)+}{ }^{2}}}=\sqrt{\frac{\hat{X}_{1(1)-}^{2}}{\hat{X}_{1(1)+}^{2}}} .
\end{aligned}
$$

We see, that they are the inverse of one another. In the respective last steps the ratio is expanded by the normalization.

The following equation gives us the possiblilty to calculate the expansions of one of these two functions (cf. eq. 4.1.13):

$$
\hat{X}_{1(i)-}^{\mu}=\hat{X}_{1(i)+}^{\mu}+2 \mathrm{i} \hat{\Theta}_{1(i)} \sigma^{\mu} \hat{\Theta}_{1(i)} .
$$


The square of this is

$$
\begin{aligned}
\hat{X}_{1(1)-}^{2} & =\hat{X}_{1(1)+}^{2}+4 i \hat{\Theta}_{1(1)} \hat{X}_{1(1)+} \hat{\bar{\Theta}}_{1(1)}-4 \hat{\Theta}_{1(1)} \sigma^{\mu} \hat{\bar{\Theta}}_{1(1)} \hat{\Theta}_{1(1)} \sigma_{\mu} \hat{\bar{\Theta}}_{1(1)} \\
& =\hat{X}_{1(1)+}^{2}+4 \mathrm{i} I_{111}-16 \frac{I_{111}^{2}}{\hat{X}_{1(1)+}^{2}}
\end{aligned}
$$

where in the second line eq. A.2.7 provides the last summand and $I_{111}$ is plugged in (cf. eq. (4.3.3)). This equation can be solved for the radicand in the last step of eq. 4.3.107). When we also use, that $\hat{X}_{1(1)-}^{2}=1 / \hat{X}_{1(1)+}^{2}$, we get

$$
\frac{\hat{X}_{1(1)-}^{2}}{\hat{X}_{1(1)+}^{2}}=1+4 \mathrm{i} I_{111} \hat{X}_{1(1)-}^{2}-16 I_{111}^{2} \hat{X}_{1(1)-}^{4} .
$$

The square root is

$$
\hat{X}_{1(1)-}^{2}=\sqrt{\frac{\hat{X}_{1(1)-}^{2}}{\hat{X}_{1(1)+}^{2}}}=1+2 \mathrm{i} I_{111} \hat{X}_{1(1)-}^{2}-6 I_{111}^{2} \hat{X}_{1(1)-}^{4},
$$

At this point we can start an iteration, which leads to the desired expansion. We plug this equation first into the first order (of $I_{111}$ ) term and then into the resulting new second order term:

$$
\hat{X}_{1(1)-}^{2}=\frac{1}{\hat{X}_{1(1)+}^{2}}=1+2 \mathrm{i} I_{111}-10 I_{111}^{2} .
$$

At the end the inverse is given by eq. A.2.10, so that the other three point variable is

$$
\hat{X}_{1(1)+}^{2}=\frac{1}{\hat{X}_{1(1)-}^{2}}=1-2 \mathrm{i} I_{111}+6 I_{111}^{2} .
$$

With eq. 4.3.112 we can also get $\hat{X}_{1(2)+}^{2}$ in terms of $\hat{X}_{1(2)-}^{2}$ and $I_{i j k}$. We rearrange eq. 4.3.108 for $i=2$ and take the square,

$$
\begin{aligned}
\hat{X}_{1(2)+}^{2} & =\hat{X}_{1(2)-}^{2}-4 \mathrm{i} I_{222}-16 \frac{I_{122}{ }^{2}}{\hat{X}_{1(1)+}} \\
& =\hat{X}_{1(2)-}^{2}-4 \mathrm{i} I_{222}-16 I_{122}^{2}-32 \mathrm{i} I_{111} I_{122}{ }^{2}+160 I_{111}^{2} I_{122}{ }^{2}
\end{aligned}
$$

The next few expansions are just the ones, which we still need to be able to switch to expansions with coefficient functions of superconformal cross ratios. We shorten the expressions with the help of

$$
\begin{aligned}
\mathfrak{X}_{1} & =\hat{X}_{1(1)+}^{\mu} \hat{X}_{1(2)-\mu}, \\
\mathfrak{X}_{2} & =\hat{X}_{1(2)-}^{2}, \\
\mathfrak{X}_{3} & =1+\hat{X}_{1(2)-}^{2}-2 \hat{X}_{1(1)+}^{\mu} \hat{X}_{1(2)-\mu} .
\end{aligned}
$$


The square of eq. 4.2 .25 is

$$
\hat{X}_{1+}^{2}=\mathfrak{X}_{3}-2 \mathrm{i} I_{111}+4 \mathrm{i} I_{112}-4 \mathrm{i} I_{212}+6 I_{111}^{2}-16 I_{112}{ }^{2} .
$$

Because the equations get longer, we introduce 36-tuple with the coefficients inside, which are defined as

$$
\left(F_{0,1}\left|F_{1,1}, \ldots, F_{1,8}\right| F_{2,1}, \ldots, F_{2,18}\left|F_{3,1}, \ldots, F_{3,8}\right| F_{4,1}\right)=\sum_{0=1}^{4} \sum_{j=1}^{n_{i}} F_{i, j} \mathfrak{I}_{i, j} .
$$

This way we can compactly write down the following results:

$$
\begin{aligned}
I_{0}= & (0|1,-1,-1,1,-1,1,1,-1| 0,-8 \mathrm{i}, 0,8 \mathrm{i}, 0,0,0,0,0,-4 \mathrm{i},-4 \mathrm{i}, 0,0,0,0 \mid 4,0,8, \\
& 0,0,0,0,0 \mid 0), \\
\hat{X}_{1-}^{2}= & \hat{X}_{1+}^{2}+4 \mathrm{i} I_{0}-16 \frac{I_{0}^{2}}{\hat{X}_{1+}^{2}} \\
= & \left(\mathfrak{X}_{3}|2 \mathrm{i}, 0,-4 \mathrm{i}, 4 \mathrm{i},-4 \mathrm{i}, 0,4 \mathrm{i},-4 \mathrm{i}|-10,0,-16,-16,0,32,0,32,0,0,0,0,-16,\right. \\
& \quad-16,0,0,0,0|-16 \mathrm{i},-32 \mathrm{i}, 0,-32 \mathrm{i}, 0,0,0,0| 160) .
\end{aligned}
$$

With the functions of three points, $\hat{X}_{1 \pm}^{2}$ and $\hat{X}_{1(i) \pm}^{2}$ we can also compute the cross ratios as such expansions:

$$
\begin{aligned}
& \mathcal{I}_{1}=\frac{X_{1-}{ }^{2}}{X_{1(2)+}{ }^{2}}=\left(\frac{\mathfrak{X}_{3}}{\mathfrak{X}_{2}}\left|\frac{2 \mathrm{i}}{\mathfrak{X}_{2}}, 0, \frac{-4 \mathrm{i}}{\mathfrak{X}_{2}}, \frac{4 \mathrm{i}}{\mathfrak{X}_{2}}, \frac{-4 \mathrm{i}}{\mathfrak{X}_{2}}, 0, \frac{4 \mathrm{i}}{\mathfrak{X}_{2}}, \frac{4 \mathrm{i}-8 \mathrm{i} \mathfrak{X}_{1}}{\mathfrak{X}_{2}{ }^{2}}\right| \frac{-10}{\mathfrak{X}_{2}}, 0, \frac{-16}{\mathfrak{X}_{2}}, \frac{-16 \mathfrak{X}_{1}}{\mathfrak{X}_{2}{ }^{2}}, 0,\right. \\
& \frac{32}{\mathfrak{X}_{2}}, 0, \frac{16 \mathfrak{X}_{2}+32 \mathfrak{X}_{1}}{\mathfrak{X}_{2}{ }^{2}}, 0,0,0, \frac{-16}{\mathfrak{X}_{2}{ }^{2}}, 0,0, \frac{-16}{\mathfrak{X}_{2}{ }^{2}}, \frac{-8}{\mathfrak{X}_{2}{ }^{2}}, 0,0 \mid 0, \frac{-32 \mathrm{i} \mathfrak{X}_{1}}{\mathfrak{X}_{2}{ }^{2}}, 0, \\
& \left.\frac{-16 \mathfrak{i}_{2}-32 \mathrm{i} \mathfrak{X}_{1}}{\mathfrak{X}_{2}{ }^{2}}, \frac{-40 \mathrm{i}}{\mathfrak{X}_{2}{ }^{2}}, 0, \frac{-64 \mathrm{i}}{\mathfrak{X}_{2}{ }^{2}}, \frac{-32 \mathrm{i}}{\mathfrak{X}_{2}{ }^{2}} \mid \frac{224 \mathfrak{X}_{1}}{\mathfrak{X}_{2}{ }^{2}}\right), \\
& \mathcal{I}_{2}=\frac{X_{1+}{ }^{2}}{X_{1(1)+}{ }^{2}}=\left(\mathfrak{X}_{3}\left|2 \mathrm{i}\left(\mathfrak{X}_{3}-1\right), 4 \mathrm{i}, 0,0,0,-4 \mathrm{i}, 0,0\right|-10\left(\mathfrak{X}_{3}-1\right),-16,0,0,-8,\right. \\
& 0,0,0,8,0,0,0,0,0,0,0,0,0|0,0,0,0,0,0,0,0| 0) \text {. }
\end{aligned}
$$

These equations can also be used to get the expressions of $\mathfrak{X}_{1}$ and $\mathfrak{X}_{2}$ in terms of these two invariants. We weaken the definition in $(4.3 .119)$ and let the coefficients also be functions of other non-nilpotent invariants. Of course, in such an expansion the coefficients are not unique, but it is useful as a shorthand notation for the purpose here. We will not distinguish the notation and use the same bracket, as it is always possible to look at the coefficients and see, which non-nilpotent invariants appear. We exchange the left-hand side of eq. (4.3.124) and the first coefficient in the bracket:

$$
-\mathfrak{X}_{3}=\left(-\mathcal{I}_{2}\left|2 \mathrm{i}\left(\mathfrak{X}_{3}-1\right), 4 \mathrm{i}, 0,0,0,-4 \mathrm{i}, 0,0\right|-10\left(\mathfrak{X}_{3}-1\right), \ldots\right) .
$$

We get to our goal with two iterative steps. We plug this expansion now into the first order coefficients in the same equation, which has influence on the higher 
coefficients after resorting the terms in the expansion. Here only one first order coefficient, $2 \mathrm{i}\left(\mathfrak{X}_{3}-1\right)$, is not constant. What we actually compute is

$$
\begin{aligned}
\mathfrak{X}_{3}= & \left(\mathcal{I}_{2}|2 \mathrm{i},-4 \mathrm{i}, 0,0,0,4 \mathrm{i}, 0,0| 10\left(\mathfrak{X}_{3}-1\right), \ldots\right) \\
& -2 \mathrm{i} I_{111}\left(\mathcal{I}_{2}\left|-2 \mathrm{i}\left(\mathfrak{X}_{3}-1\right),-4 \mathrm{i}, 0,0,0,4 \mathrm{i}, 0,0\right| 10\left(\mathfrak{X}_{3}-1\right), \ldots\right) \\
= & \left(\mathcal{I}_{2}\left|2 \mathrm{i}\left(1-\mathcal{I}_{2}\right),-4 \mathrm{i}, 0,0,0,4 \mathrm{i}, 0,0\right| 6\left(\mathfrak{X}_{3}-1\right), 16,0,0,0,0,0,0,\right. \\
& 0,0,0,0,0,0,0,0,0,0|0,0,0,0,0,0,0,0| 0) .
\end{aligned}
$$

If we plug this into the coefficient of $I_{111}{ }^{2}$, we see that only the zeroth order coefficient survives in the product with $I_{111}^{2}$ :

$$
\begin{aligned}
\mathfrak{X}_{3}= & \left(\mathcal{I}_{2}\left|2 \mathrm{i}\left(1-\mathcal{I}_{2}\right),-4 \mathrm{i}, 0,0,0,4 \mathrm{i}, 0,0\right| 6\left(\mathcal{I}_{2}-1\right), 16,0,0,0,0,0,0,\right. \\
& 0,0,0,0,0,0,0,0,0,0|0,0,0,0,0,0,0,0| 0) .
\end{aligned}
$$

This is the first half on the way to the expansion with coefficient functions of two cross ratios. Eq. 4.3.123) is the starting point for the other half. We calculate the inverse with the help of eq. (D.4.1) and get

$$
\begin{aligned}
\frac{1}{\mathcal{I}_{1}}= & \left(\frac{\mathfrak{X}_{2}}{\mathfrak{X}_{3}}\left|\frac{-2 \mathrm{i} \mathfrak{X}_{2}}{\mathfrak{X}_{3}{ }^{2}}, 0, \frac{4 \mathrm{i} \mathfrak{X}_{2}}{\mathfrak{X}_{3}{ }^{2}}, \frac{-4 \mathrm{i} \mathfrak{X}_{2}}{\mathfrak{X}_{3}{ }^{2}}, \frac{4 \mathrm{i} \mathfrak{X}_{2}}{\mathfrak{X}_{3}{ }^{2}}, 0, \frac{-4 \mathrm{i} \mathfrak{X}_{2}}{\mathfrak{X}_{3}{ }^{2}}, \frac{4 \mathrm{i}\left(-1+2 \mathfrak{X}_{1}\right)}{\mathfrak{X}_{3}{ }^{2}}\right|\right. \\
& 2 \frac{\mathfrak{X}_{2}\left(2-4 \mathfrak{X}_{2}+\mathfrak{X}_{3}\right)}{\mathfrak{X}_{3}{ }^{3}}, 0,0,16 \frac{-1+\mathfrak{X}_{1}}{\mathfrak{X}_{3}{ }^{2}}, 0,-16 \frac{\mathfrak{X}_{2}}{\mathfrak{X}_{3}{ }^{3}}, 0,16 \frac{1-\mathfrak{X}_{3}}{\mathfrak{X}_{3}{ }^{2}}, 0, \\
& 16 \frac{\mathfrak{X}_{2}}{\mathfrak{X}_{3}{ }^{3}}, 0,-16 \frac{1}{\mathfrak{X}_{3}{ }^{2}},-16 \frac{\mathfrak{X}_{2}\left(-1+4 \mathfrak{X}_{1}\right)}{\mathfrak{X}_{3}{ }^{3}},-32 \frac{\mathfrak{X}_{2}\left(-1+2 \mathfrak{X}_{1}\right)}{\mathfrak{X}_{3}{ }^{3}}, \\
& 16 \frac{2 \mathfrak{X}_{2}-\mathfrak{X}_{3}}{\mathfrak{X}_{3}{ }^{3}}, 8 \frac{2 \mathfrak{X}_{2}-\mathfrak{X}_{3}}{\mathfrak{X}_{3}{ }^{3}}, 0,32 \frac{\mathfrak{X}_{2}}{\mathfrak{X}_{3}{ }^{3}} \mid \frac{16 \mathfrak{X}_{2}\left(2+7 \mathfrak{X}_{2}-\left(6-4 \mathfrak{X}_{3}\right) \mathfrak{X}_{1}\right)}{\mathfrak{X}_{3}{ }^{4}} \\
& \frac{32 \mathrm{i}\left(\left(2+\mathfrak{X}_{3}\right) \mathfrak{X}_{1}-2 \mathfrak{X}_{2}\right)}{\mathfrak{X}_{3}{ }^{3}}, 0, \frac{16 \mathrm{i}\left(3 \mathfrak{X}_{2}+\mathfrak{X}_{2}{ }^{2}-2\left(\mathfrak{X}_{3}+\mathfrak{X}_{2}\right) \mathfrak{X}_{1}\right)}{\mathfrak{X}_{3}{ }^{3}}, \\
& \frac{-8 \mathrm{i}\left(3-12 \mathfrak{X}_{2}+\left(16 \mathfrak{X}_{2}-8\right) \mathfrak{X}_{1}+3 \mathfrak{X}_{2}{ }^{2}+4 \mathfrak{X}_{1}{ }^{2}\right)}{\mathfrak{X}_{3}{ }^{4}}, \\
& 0, \frac{-32 \mathrm{i}}{\mathfrak{X}_{3}{ }^{3}}, \frac{32 \mathrm{i}\left(-3+\mathfrak{X}_{2}+2 \mathfrak{X}_{1}\right)}{\mathfrak{X}_{3}{ }^{3}} \mid \\
& \left.\frac{\left.-32\left(12 \mathfrak{X}_{1}{ }^{3}-\left(24+20 \mathfrak{X}_{2}\right) \mathfrak{X}_{1}{ }^{2}+\left(36 \mathfrak{X}_{2}+15+7 \mathfrak{X}_{2}{ }^{2}\right) \mathfrak{X}_{1}-22 \mathfrak{X}_{2}-4 \mathfrak{X}_{2}{ }^{2}\right)\right)}{\mathfrak{X}_{3}{ }^{4}}\right) .
\end{aligned}
$$

For this problem we programmed a Maple procedure (cf. D.2), which can replace $\mathfrak{X}_{2}$ by any invariant, in which the zeroth order is a monomial, in which $\mathfrak{X}_{2}$ appears with the degree 1 . This is the case here. So the procedure subtracts the zeroth order term and the left hand side, so that they exchange places and divides by the factor of $\mathfrak{X}_{2}-$ here $1 / \mathfrak{X}_{3}-$, which is now on the left hand side. This way it leaves $\mathfrak{X}_{2}$ there. Then it substitutes

$$
\mathfrak{X}_{1}=\frac{1}{2}\left(1+\mathfrak{X}_{2}-\mathfrak{X}_{3}\right),
$$

and inserts eq. 4.3.127) everywhere in the right power. Furthermore it goes through the iterative steps to replace all $\mathfrak{X}_{2}$ by the current intermediate result. 
The final result is

$$
\begin{aligned}
\hat{\mathrm{X}}_{1(2)-}^{2}= & \left(\frac{\mathcal{I}_{2}}{\mathcal{I}_{1}}\left|2 \mathrm{i} \frac{2-\mathcal{I}_{2}}{\mathcal{I}_{1}},-\frac{4 \mathrm{i}}{\mathcal{I}_{1}},-\frac{4 \mathrm{i}}{\mathcal{I}_{1}}, \frac{4 \mathrm{i}}{\mathcal{I}_{1}},-\frac{4 \mathrm{i}}{\mathcal{I}_{1}}, \frac{4 \mathrm{i}}{\mathcal{I}_{1}}, \frac{4 \mathrm{i}}{\mathcal{I}_{1}}, 4 \mathrm{i} \frac{\mathcal{I}_{1}-1}{\mathcal{I}_{1}}\right| \frac{6 \mathcal{I}_{2}-16}{\mathcal{I}_{1}}, \frac{16}{\mathcal{I}_{1}},\right. \\
& -\frac{16}{\mathcal{I}_{1}}, 16 \frac{\mathcal{I}_{1}-1}{\mathcal{I}_{1}}, 0, \frac{32}{\mathcal{I}_{1}}, 0, \frac{32}{\mathcal{I}_{1}}, 0,0,0,0,-\frac{16}{\mathcal{I}_{1}},-\frac{16}{\mathcal{I}_{1}}, 0,0,0,0 \mid \\
& \left.-\frac{16 \mathrm{i}}{\mathcal{I}_{1}},-32 \mathrm{i} \frac{1-\mathcal{I}_{1}}{\mathcal{I}_{1}}, 0,-\frac{32 \mathrm{i}}{\mathcal{I}_{1}}, 0,0,0,0 \mid 160 \frac{1-\mathcal{I}_{1}}{\mathcal{I}_{1}}\right) \\
= & \frac{\mathcal{I}_{2}}{\mathcal{I}_{1}}-2 \mathrm{i} \frac{\mathcal{I}_{2}}{\mathcal{I}_{1}} I_{111}+4 \mathrm{i} \frac{1}{\mathcal{I}_{1}} I_{0}+4 \mathrm{i} I_{222}+6 \frac{\mathcal{I}_{2}}{\mathcal{I}_{1}} I_{111}{ }^{2}-16 \frac{1}{\mathcal{I}_{1} \mathcal{I}_{2}} I_{0}{ }^{2}+16 \frac{\mathcal{I}_{1}}{\mathcal{I}_{2}} I_{222}{ }^{2} \\
& +32 \mathrm{i} \frac{\mathcal{I}_{1}}{\mathcal{I}_{2}} I_{111} I_{222}{ }^{2}-64 \mathrm{i} \frac{\mathcal{I}_{1}}{\mathcal{I}_{2}{ }^{2}} I_{222}{ }^{2} I_{0}+32 \frac{\mathcal{I}_{2}+4 \mathcal{I}_{1}-\mathcal{I}_{1} \mathcal{I}_{2}}{\mathcal{I}_{2}{ }^{2}} I_{111}{ }^{2} I_{222}{ }^{2} .(4.3 .1
\end{aligned}
$$

With this equation and eq. 4.3.127) any invariant, which is given in form of eq. D.1.1) can now be transformed into the expansion

$$
\begin{aligned}
& \check{F}\left(\mathcal{I}_{1}, \mathcal{I}_{2}, I_{111}, \ldots, I_{222}\right) \\
& =\sum_{i=0}^{4} \sum_{j=1}^{n_{i}} \check{F}_{i, j}\left(\mathcal{I}_{1}, \mathcal{I}_{1}\right) \mathfrak{I}_{i, j} \text {. }
\end{aligned}
$$

The procedure, which does this job, is similar to the one we used to get eq. 4.3.129) (cf. appendix D.3). This can then be applied to the product of two unknown invariants in eqns. D.1.5)-D.1.8 with $F_{i, j}$ and $G_{i, j}$ replaced by the $\check{F}_{i, j}$ and $\check{G}_{i, j}$ and results in the equations of the coefficient functions $\check{H}_{i, j}\left(\mathcal{I}_{1}, \mathcal{I}_{1}\right)$ of the product $\check{H}=\check{F} \check{G}$.

With the equations and procedures to calculate the inverse and the square root (cf. D.4) one can compute the expansions of all invariants, which we encounter in this thesis. In eqns. 4.3.112 and 4.3.113 we had already $\hat{\mathrm{X}}_{1(1)+}^{2}$ and $\hat{\mathrm{X}}_{1(1)-}^{2}=$ $1 / \hat{\mathrm{X}}_{1(1)+}^{2}$. For $\hat{\mathrm{X}}_{1+}^{2}$ we can use 4.2.7):

$$
\begin{aligned}
\hat{\mathrm{X}}_{1+}^{2} & =\hat{\mathrm{X}}_{1(1)+}^{2} \mathcal{I}_{2} \\
& =\mathcal{I}_{2}-2 \mathrm{i} \mathcal{I}_{2} I_{111}+6 \mathcal{I}_{2} I_{111}{ }^{2} .
\end{aligned}
$$

The inverse is then, of course, proportional to $\hat{\mathrm{X}}_{1(1)-}^{2}$. If we look at further functions of three points it is often shorter to write them with the help of $I_{0}$, as in eq. 4.3.130:

$$
\begin{aligned}
\hat{\mathrm{X}}_{1(2)+}^{2}= & \frac{\mathcal{I}_{2}}{\mathcal{I}_{1}}-2 \mathrm{i} \frac{\mathcal{I}_{2}}{\mathcal{I}_{1}} I_{111}+4 \mathrm{i} \frac{1}{\mathcal{I}_{1}} I_{0}+6 \frac{\mathcal{I}_{2}}{\mathcal{I}_{1}} I_{111}{ }^{2}-16 \frac{1}{\mathcal{I}_{1} \mathcal{I}_{2}} I_{0}{ }^{2} \\
& -32 \mathrm{i} \frac{1}{\mathcal{I}_{1} \mathcal{I}_{2}} I_{112}{ }^{2} I_{121}+160 \frac{1}{\mathcal{I}_{2}} I_{111}{ }^{2} I_{222}{ }^{2}, \\
\frac{1}{\hat{\mathrm{X}}_{1(2)+}^{2}}= & \frac{\mathcal{I}_{1}}{\mathcal{I}_{2}}+2 \mathrm{i} \frac{\mathcal{I}_{1}}{\mathcal{I}_{2}} I_{111}-4 \mathrm{i} \frac{\mathcal{I}_{1}}{\mathcal{I}_{2}{ }^{2}} I_{0}-10 \frac{\mathcal{I}_{1}}{\mathcal{I}_{2}} I_{111}{ }^{2}+16 \frac{\mathcal{I}_{1}}{\mathcal{I}_{2}{ }^{2}} I_{111} I_{0} \\
& +96 \mathrm{i} \frac{\mathcal{I}_{1}}{\mathcal{I}_{2}{ }^{2}} I_{111}{ }^{2} I_{0},
\end{aligned}
$$




$$
\begin{aligned}
\frac{1}{\hat{\mathrm{X}}_{1(2)-}^{2}}= & \frac{\mathcal{I}_{1}}{\mathcal{I}_{2}}+2 \mathrm{i} \frac{\mathcal{I}_{1}}{\mathcal{I}_{2}} I_{111}-4 \mathrm{i} \frac{\mathcal{I}_{1}}{\mathcal{I}_{2}{ }^{2}} I_{0}-4 \mathrm{i} \frac{\mathcal{I}_{1}{ }^{2}}{\mathcal{I}_{2}{ }^{2}} I_{0}-10 \frac{\mathcal{I}_{1}}{\mathcal{I}_{2}} I_{111}{ }^{2}-32 \frac{\mathcal{I}_{1}{ }^{3}}{\mathcal{I}_{2}{ }^{3}} I_{222}^{2} \\
& +16 \frac{\mathcal{I}_{1}}{\mathcal{I}_{2}{ }^{2}} I_{111} I_{0}+16 \frac{\mathcal{I}_{1}{ }^{2}}{\mathcal{I}_{2}{ }^{2}} I_{111} I_{222}-32 \frac{\mathcal{I}_{1}{ }^{2}}{\mathcal{I}_{2}{ }^{3}} I_{0} I_{222}+96 \mathrm{i} \frac{\mathcal{I}_{1}}{\mathcal{I}_{2}{ }^{3}} I_{111} I_{0}{ }^{2} \\
& -32 \mathrm{i} \frac{\mathcal{I}_{1}{ }^{2}}{\mathcal{I}_{2}{ }^{4}} I_{222} I_{0}{ }^{2}-96 \mathrm{i} \frac{\mathcal{I}_{1}{ }^{3}}{\mathcal{I}_{2}{ }^{3}} I_{111} I_{222}{ }^{2}+288 \mathrm{i} \frac{\mathcal{I}_{1}{ }^{3}}{\mathcal{I}_{2}{ }^{4}} I_{0} I_{222}{ }^{2} \\
& +32 \frac{\mathcal{I}_{1}{ }^{2}\left(\mathcal{I}_{1}+7 \mathcal{I}_{2}-7 \mathcal{I}_{1} \mathcal{I}_{2}\right)}{\mathcal{I}_{2}{ }^{4}} I_{111}{ }^{2} I_{222}{ }^{2}
\end{aligned}
$$

With all these squares of functions of three points, their inverses and a procedure to calculate their products one quickly gets to the three point invariants $J_{1}$ and $J_{1(i)}$ and the other cross ratios $\mathcal{I}_{i}$.

$$
\begin{aligned}
& J_{1}=1-4 \mathrm{i} \frac{1}{\mathcal{I}_{2}} I_{0}+\frac{8}{\mathcal{I}_{2}} I_{111} I_{0}+40 \mathrm{i} \frac{1}{\mathcal{I}_{2}} I_{111}^{2} I_{0}, \\
& J_{1(1)}=1-4 \mathrm{i} I_{111}+8 I_{111}{ }^{2} \text {, } \\
& J_{1(2)}=1-4 \mathrm{i} \frac{\mathcal{I}_{1}}{\mathcal{I}_{2}} I_{222}-32 \frac{\mathcal{I}_{1}{ }^{2}}{\mathcal{I}_{2}{ }^{2}} I_{222}^{2}+8 \frac{\mathcal{I}_{1}}{\mathcal{I}_{2}} I_{111} I_{222}-16 \frac{\mathcal{I}_{1}}{\mathcal{I}_{2}{ }^{2}} I_{0} I_{222}+8 \mathrm{i} \frac{\mathcal{I}_{1}}{\mathcal{I}_{2}} I_{111}{ }^{2} I_{222} \\
& +32 \mathrm{i} \frac{1}{\mathcal{I}_{2}{ }^{2}} I_{111} I_{0}{ }^{2}-32 \mathrm{i} \frac{\mathcal{I}_{1}}{\mathcal{I}_{2}{ }^{3}} I_{222} I_{0}{ }^{2}-96 \mathrm{i} \frac{\mathcal{I}_{1}{ }^{2}}{\mathcal{I}_{2}{ }^{2}} I_{111} I_{222}{ }^{2}+224 \mathrm{i} \frac{\mathcal{I}_{1}{ }^{2}}{\mathcal{I}_{2}{ }^{3}} I_{0} I_{222}{ }^{2} \\
& -64 \frac{\mathcal{I}_{1}\left(3 \mathcal{I}_{1}-7 \mathcal{I}_{2}+\mathcal{I}_{1} \mathcal{I}_{2}\right)}{\mathcal{I}_{2}{ }^{3}} I_{111}{ }^{2} I_{222}{ }^{2} \\
& \mathcal{I}_{3}=\mathcal{I}_{2}-4 \mathrm{i} \mathcal{I}_{2} I_{111}+4 \mathrm{i} I_{0}+8 \mathcal{I}_{2} I_{111}{ }^{2}-\frac{16}{\mathcal{I}_{2}} I_{0}{ }^{2}+8 I_{111} I_{0}+24 \mathrm{i} I_{111}^{2} I_{0}, \\
& \mathcal{I}_{4}=\frac{\mathcal{I}_{1}}{\mathcal{I}_{2}}-4 \mathrm{i} \frac{\mathcal{I}_{1}}{\mathcal{I}_{2}{ }^{2}} I_{0}+8 \frac{\mathcal{I}_{1}}{\mathcal{I}_{2}{ }^{2}} I_{111} I_{0}+40 \mathrm{i} \frac{\mathcal{I}_{1}}{\mathcal{I}_{2}{ }^{2}} I_{111}{ }^{2} I_{0}, \\
& \mathcal{I}_{5}=\mathcal{I}_{1}-4 \mathrm{i} \frac{\mathcal{I}_{1}}{\mathcal{I}_{2}} I_{0}-4 \mathrm{i} \frac{\mathcal{I}_{1}{ }^{2}}{\mathcal{I}_{2}} I_{222}-32 \frac{\mathcal{I}_{1}{ }^{3}}{\mathcal{I}_{2}{ }^{2}} I_{222}^{2}+8 \frac{\mathcal{I}_{1}}{\mathcal{I}_{2}} I_{111} I_{0}+8 \frac{\mathcal{I}_{1}{ }^{2}}{\mathcal{I}_{2}} I_{111} I_{222} \\
& -32 \frac{\mathcal{I}_{1}^{2}}{\mathcal{I}_{2}{ }^{2}} I_{0} I_{222}-24 \mathrm{i} \frac{\mathcal{I}_{1}}{\mathcal{I}_{2}} I_{111}{ }^{2} I_{0}-24 \mathrm{i} \frac{\mathcal{I}_{1}{ }^{2}}{\mathcal{I}_{2}} I_{111}{ }^{2} I_{222}+64 \mathrm{i} \frac{\mathcal{I}_{1}}{\mathcal{I}_{2}{ }^{2}} I_{111} I_{0}{ }^{2} \\
& -64 \mathrm{i} \frac{\mathcal{I}_{1}{ }^{3}}{\mathcal{I}_{2}{ }^{2}} I_{111} I_{222}{ }^{2}+320 \mathrm{i} \frac{\mathcal{I}_{1}{ }^{3}}{\mathcal{I}_{2}{ }^{3}} I_{0} I_{222}{ }^{2}+320 \frac{\mathcal{I}_{1}{ }^{2}\left(\mathcal{I}_{1}+\mathcal{I}_{2}-\mathcal{I}_{1} \mathcal{I}_{2}\right)}{\mathcal{I}_{2}{ }^{3}} I_{111}{ }^{2} I_{222}{ }^{2}, \\
& \mathcal{I}_{6}=\frac{\mathcal{I}_{1}}{\mathcal{I}_{2}}-4 \mathrm{i} \frac{\mathcal{I}_{1}}{\mathcal{I}_{2}} I_{111}-4 \mathrm{i} \frac{\mathcal{I}_{1}}{\mathcal{I}_{2}{ }^{2}} I_{0}-4 \mathrm{i} \frac{\mathcal{I}_{1}{ }^{2}}{\mathcal{I}_{2}{ }^{2}} I_{222}-24 \frac{\mathcal{I}_{1}}{\mathcal{I}_{2}} I_{111}^{2}-32 \frac{\mathcal{I}_{1}^{3}}{\mathcal{I}_{2}{ }^{3}} I_{222}^{2}+24 \frac{\mathcal{I}_{1}}{\mathcal{I}_{2}{ }^{2}} I_{111} I_{0} \\
& +24 \frac{\mathcal{I}_{1}^{2}}{\mathcal{I}_{2}{ }^{2}} I_{111} I_{222}-32 \frac{\mathcal{I}_{1}{ }^{2}}{\mathcal{I}_{2}{ }^{3}} I_{0} I_{222}+40 \mathrm{i} \frac{\mathcal{I}_{1}}{\mathcal{I}_{2}{ }^{2}} I_{111}{ }^{2} I_{0}+40 \mathrm{i} \frac{\mathcal{I}_{1}{ }^{2}}{\mathcal{I}_{2}{ }^{2}} I_{111}{ }^{2} I_{222} \\
& +128 \mathrm{i} \frac{\mathcal{I}_{1}}{\mathcal{I}_{2}{ }^{3}} I_{111} I_{0}{ }^{2}-64 \mathrm{i} \frac{\mathcal{I}_{1}{ }^{2}}{\mathcal{I}_{2}{ }^{4}} I_{111} I_{0}{ }^{2}-128 \mathrm{i} \frac{\mathcal{I}_{1}{ }^{3}}{\mathcal{I}_{2}{ }^{3}} I_{111} I_{222}{ }^{2}+256 \mathrm{i} \frac{\mathcal{I}_{1}{ }^{3}}{\mathcal{I}_{2}{ }^{4}} I_{0} I_{222}{ }^{2} \\
& -64 \frac{\mathcal{I}_{1}^{2}\left(3 \mathcal{I}_{1}-\mathcal{I}_{2}+\mathcal{I}_{1} \mathcal{I}_{2}\right)}{\mathcal{I}_{2}{ }^{4}} I_{111}{ }^{2} I_{222}{ }^{2}
\end{aligned}
$$

It is remarkable, that all expansions in this section with coefficient functions of the 
cross ratios except $\mathfrak{X}_{3}$ can be rewritten in a form, in which they only contain three nilpotent invariants, $I_{111}, I_{0}$ and $I_{222}$. They all have in common, that they are ratios of squared superconformal intervals, $x_{i j}{ }^{2}$, or the square root of such ratios.

\subsubsection{Alternative nilpotent invariants}

In the last section we have encountered already the invariant $I_{0}$, which is a function of all $I_{i j k}$ and has an especially simple structure. This invariant, $I_{111}$ and $I_{222}$ differ only in their indices apart from their normalization, which is the same for all three. To simplify calculations one can replace any of the other six $I_{i j k}$ by $I_{0}$. We will see that we are led automatically to this replacement in our discussion of the four point function.

If we look at the form of $I_{111}$ in eq. 4.3.10, we find another possibility to get an even simpler invariant looking at the mixed terms of second order in $\rho$ 's. These also appear in $I_{111}^{2}$. So one can replace them by squares of $\rho$ 's by computing

$$
\begin{aligned}
T_{111}= & \frac{I_{111}}{\sqrt{J_{1(1)}}}+2 \mathrm{i} \frac{I_{111}^{2}}{J_{1(1)}} \\
= & -\rho_{41}-\rho_{12}-\rho_{24}+2 \mathrm{i}\left(\rho_{41}^{2}+\rho_{12}^{2}+\rho_{24}^{2}\right) \\
& -16\left(\rho_{24}^{2} \rho_{12}+\rho_{24} \rho_{12}^{2}+\rho_{24}^{2} \rho_{41}+\rho_{24} \rho_{41}^{2}+\rho_{41}^{2} \rho_{12}+\rho_{41} \rho_{12}^{2}+2 \rho_{24} \rho_{12} \rho_{12}\right) \\
& -32 \mathrm{i}\left(\rho_{24}^{2} \rho_{12}^{2}+\rho_{41}^{2} \rho_{12}^{2}+\rho_{24}^{2} \rho_{41}^{2}+4 \rho_{12} \rho_{24} \rho_{41}^{2}+4 \rho_{12} \rho_{24}^{2} \rho_{41}+4 \rho_{12}^{2} \rho_{24} \rho_{41}\right) .
\end{aligned}
$$

We devide by the factor in eq. 4.3.10, which is just $\sqrt{J_{1(1)}}$, and the corresonding square in the first line.

With the equations 4.3.14-4.3.17), which we have found on the way to the expression of $I_{111}$ in terms of $\rho$ 's (eq. (4.3.18)), we also get a simple result for $T_{111}$, which is certainly does not contain any vanishing higher degree terms, because here all of these have already disappeared:

$$
T_{111}=-\rho_{41}-\rho_{12}-\rho_{24}+2 \mathrm{i}\left(\rho_{41}^{2}+\rho_{12}^{2}+\rho_{24}^{2}\right) .
$$

This invariant has not only a very short expression in terms of the superconformal intervals, but also has the property, that only signs change, if one switches the indices of the $\rho$ 's (cf. once more eq. A.2.11). For the expansion of this invariant directly in terms of our standard set of invariants we insert eq. 4.3.112 into its definition and get

$$
\begin{aligned}
T_{111} & =\hat{X}_{1(1)-}^{2} I_{111}+2 \mathrm{i} \hat{X}_{1(1)-}^{4} I_{111}^{2} \\
& =I_{111}+4 \mathrm{i} I_{111}{ }^{2} .
\end{aligned}
$$

The factor $X_{1(1)-}{ }^{4}$ in the second summand just reduces to 1 because it is multiplied by $I_{111}^{2}$.

Of course, we can get other invariants of this kind by replacing the indices of the points this one depends on. If we replace the index 2 by 3 , we get an invariant, 
which we can write down in terms of $I_{222}$ and $\hat{X}_{1(2)+}^{2}$ :

$$
\begin{aligned}
T_{222} & =\frac{1}{\hat{X}_{1(2)+}^{2}} I_{222}+2 \mathrm{i} \frac{1}{\hat{X}_{1(2)+}^{4}} I_{222}^{2} \\
& =-\rho_{41}-\rho_{13}-\rho_{34}+2 \mathrm{i}\left(\rho_{41}{ }^{2}+\rho_{13}{ }^{2}+\rho_{34}{ }^{2}\right) .
\end{aligned}
$$

Here we inserted into eq. 4.3.145, that $\hat{X}_{1(1)+}^{2}$ is the inverse of $\hat{X}_{1(1)-}^{2}$. Then there is a quotient of $I_{111}$ and $\hat{X}_{1(1)+}^{2}$ and the normalizations cancel. The indices can be replaced and the ratio can be expanded by the normalization again leading to the just stated result.

If one replaces instead the index 4 by 3 , one gets the third invariant of this kind, which we will later need:

$$
\begin{aligned}
T_{0} & =\frac{1}{\hat{X}_{1+}^{2}} I_{0}+2 \mathrm{i} \frac{1}{\hat{X}_{1+}^{4}} I_{0}^{2} \\
& =\rho_{13}-\rho_{12}-\rho_{23}+2 \mathrm{i}\left(\rho_{12}{ }^{2}+\rho_{23}{ }^{2}-\rho_{13}{ }^{2}\right) .
\end{aligned}
$$

A bit more complex is the case of the fourth invariant, which we need in the expression of the four-point function, which we show in the next section. We start from the nilpotent invariant $I_{212}$ and expand its definition 4.3.4 with the help of eq. A.2.3.

$$
\begin{aligned}
& I_{212}=\frac{\frac{x_{\overline{4}}{ }^{2}}{\bar{x}_{\overline{4} 1}{ }^{2} x_{\overline{1} 3}{ }^{2}}}{\sqrt{X_{1(1)+}{ }^{2} X_{1(1)-}{ }^{2}}} \quad\left(\theta_{43} \tilde{x}_{\overline{4} 3}^{-1}-\theta_{14} \tilde{x}_{\overline{1} 4}^{-1}+4 \mathrm{i} \theta_{14} \tilde{x}_{\overline{1} 4}^{-1} \bar{\theta}_{14} \theta_{43} \tilde{x}_{\overline{4} 3}^{-1}\right) \tilde{x}_{\overline{4} 1} \\
& \left(\tilde{x}_{\overline{4} 1}^{-1} \bar{\theta}_{41}-\tilde{x}_{\overline{2} 1}^{-1} \bar{\theta}_{21}\right) \text {. }
\end{aligned}
$$

The factor in front of the first bracket is $\hat{X}_{1(2)-}^{2}$. We devide by it here and find the following structure:

$$
\begin{aligned}
\frac{I_{212}}{\hat{X}_{1(2)-}^{2}}= & \rho_{14}+\left[\theta_{43} \tilde{x}_{\overline{4} 3}^{-1} \tilde{x}_{\overline{4} 1}\left(\tilde{x}_{\overline{4} 1}^{-1} \bar{\theta}_{41}-\tilde{x}_{\overline{2} 1}^{-1} \bar{\theta}_{21}\right)-\theta_{14} \tilde{x}_{\overline{2} 1}^{-1} \bar{\theta}_{21}\right] \\
& +4 \mathrm{i} \rho_{14}\left[\theta_{43} \tilde{x}_{\overline{4} 3}^{-1} \tilde{x}_{\overline{4} 1}\left(\tilde{x}_{\overline{4} 1}^{-1} \bar{\theta}_{41}-\tilde{x}_{\overline{2} 1}^{-1} \bar{\theta}_{21}\right)-\theta_{14} \tilde{x}_{\overline{2} 1}^{-1} \bar{\theta}_{21}\right] .
\end{aligned}
$$

The two square brackets are identical and we define $-t_{212}$ to be this square bracket. We continue to expand it and use in the term, which contains $\tilde{x}_{\overline{2} 1}^{-1}$ and $\tilde{x}_{\overline{4} 3}^{-1}$, that

$$
\tilde{x}_{\overline{4} 1}=\tilde{x}_{\overline{2} 1}+\tilde{x}_{\overline{4} 3}-\tilde{x}_{\overline{2} 3}-4 \mathrm{i} \bar{\theta}_{42} \theta_{31} .
$$

We finally find, that

$$
\begin{aligned}
\frac{I_{212}}{\hat{X}_{1(2)-}^{2}}= & \rho_{14}-t_{212}-4 \mathrm{i} \rho_{14} t_{212} \\
t_{212}= & \theta_{43} \tilde{x}_{\overline{4} 3}^{-1} \bar{\theta}_{24}+\theta_{13} \tilde{x}_{\overline{2} 1}^{-1} \bar{\theta}_{21}-\theta_{43} \tilde{x}_{\overline{4} 3}^{-1} \tilde{x}_{\overline{2} 3} \tilde{x}_{\overline{2} 1}^{-1} \bar{\theta}_{21} \\
& -4 \mathrm{i} \theta_{43} \tilde{x}_{\overline{4} 3}^{-1} \bar{\theta}_{24} \theta_{13} \tilde{x}_{\overline{2} 1}^{-1} \bar{\theta}_{21} .
\end{aligned}
$$


Now we define the invariant, which we are ultimately interested in. We eliminate the mixed term $\rho_{14} t_{212}$ by subtracting a multiple of the square of this invariant:

$$
\begin{aligned}
T_{212}=\frac{I_{212}}{\hat{X}_{1(2)-}^{2}}-2 \mathrm{i} \frac{I_{212}{ }^{2}}{\hat{X}_{1(2)-}^{4}}= & \rho_{14}-t_{212}-2 \mathrm{i}\left(\rho_{14}{ }^{2}+t_{212}{ }^{2}\right) \\
& -16\left(\rho_{14}{ }^{2} t_{212}-\rho_{14} t_{212}{ }^{2}\right)+32 \mathrm{i} \rho_{14}{ }^{2} t_{212}{ }^{2} .
\end{aligned}
$$

At this point we can use the higher powers of $T_{212}$, which vanish, to simplify the expression here. With the help of computer algebra this can be done quite easily. We have declared the three terms in $t_{212}$ and $\rho_{14}$ as variables, but not resolved their structure. Naturally, one then has to demand a lot of conditions, that none of them appears with a power, that is too high. These have been already sixteen conditions like third powers or

$$
\begin{aligned}
\left(\theta_{43} \tilde{x}_{\overline{4} 3}^{-1} \bar{\theta}_{24}\right)^{2} \theta_{43} \tilde{x}_{\overline{4} 3}^{-1} \tilde{x}_{\overline{2} 3} \tilde{x}_{\overline{2} 1}^{-1} \bar{\theta}_{21} & =0, \\
\left(\theta_{43} \tilde{x}_{\overline{4} 3}^{-1} \bar{\theta}_{24}\right)^{2} \rho_{14}\left(\theta_{13} \tilde{x}_{\overline{2} 1}^{-1} \bar{\theta}_{21}\right)^{2} & =0, \\
\theta_{43} \tilde{x}_{\overline{4} 3}^{-1} \bar{\theta}_{24} \rho_{14}^{2} \theta_{13} \tilde{x}_{\overline{2} 1}^{-1} \bar{\theta}_{21} \theta_{43} \tilde{x}_{\overline{4} 3}^{-1} \tilde{x}_{\overline{2} 3} \tilde{x}_{\overline{2} 1}^{-1} \bar{\theta}_{21} & =0 .
\end{aligned}
$$

The forth power of $T_{212}$ is computed to be

$$
\begin{aligned}
T_{212}{ }^{4}= & 6\left[\rho_{14}^{2}\left(\theta_{13} \tilde{x}_{\overline{2} 1}^{-1} \bar{\theta}_{21}\right)^{2}+\left(\theta_{13} \tilde{x}_{\overline{2} 1}^{-1} \bar{\theta}_{21}\right)^{2}\left(\theta_{43} \tilde{x}_{\overline{4} 3}^{-1} \bar{\theta}_{24}\right)^{2}\right. \\
& +\rho_{14}{ }^{2}\left(\theta_{43} \tilde{x}_{\overline{4} 3}^{-1} \tilde{x}_{\overline{2} 3} \tilde{x}_{\overline{2} 1}^{-1} \bar{\theta}_{21}\right)^{2}+\rho_{14}^{2} \theta_{43} \tilde{x}_{\overline{4} 3}^{-1} \bar{\theta}_{24}{ }^{2}-2\left(\theta_{13} \tilde{x}_{\overline{2} 1}^{-1} \bar{\theta}_{21}\right)^{2} \rho_{14} \theta_{43} \tilde{x}_{\overline{4} 3}^{-1} \bar{\theta}_{24} \\
& -2 \theta_{13} \tilde{x}_{\overline{2} 1}^{-1} \bar{\theta}_{21} \rho_{14}{ }^{2} \theta_{43} \tilde{x}_{\overline{4} 3}^{-1} \tilde{x}_{\overline{2} 3} \tilde{x}_{\overline{2} 1}^{-1} \bar{\theta}_{21}-2 \theta_{13} \tilde{x}_{\overline{2} 1}^{-1} \bar{\theta}_{21} \rho_{14}{ }^{2} \theta_{43} \tilde{x}_{\overline{4} 3}^{-1} \bar{\theta}_{24} \\
& -2 \theta_{13} \tilde{x}_{\overline{2} 1}^{-1} \bar{\theta}_{21} \rho_{14}\left(\theta_{43} \tilde{x}_{\overline{4} 3}^{-1} \bar{\theta}_{24}\right)^{2}-2 \rho_{14}{ }^{2} \theta_{43} \tilde{x}_{\overline{4} 3}^{-1} \bar{\theta}_{24} \theta_{43} \tilde{x}_{\overline{4} 3}^{-1} \tilde{x}_{\overline{2} 3} \tilde{x}_{\overline{2} 1}^{-1} \bar{\theta}_{21} \\
& \left.+4 \theta_{13} \tilde{x}_{\overline{2} 1}^{-1} \bar{\theta}_{21} \rho_{14} \theta_{43} \tilde{x}_{\overline{4} 3}^{-1} \bar{\theta}_{24} \theta_{43} \tilde{x}_{\overline{4} 3}^{-1} \tilde{x}_{\overline{2} 3} \tilde{x}_{\overline{2} 1}^{-1} \bar{\theta}_{21}\right] \\
= & 0
\end{aligned}
$$

As written in the last line this has to be zero just as the next result, which uses this one already:

$$
\begin{aligned}
T_{212}{ }^{3} \rho_{14}= & 3\left[\theta_{13} \tilde{x}_{\overline{2} 1}^{-1} \bar{\theta}_{21} \rho_{14}\left(\theta_{43} \tilde{x}_{\overline{4} 3}^{-1} \bar{\theta}_{24}\right)^{2}+\left(\theta_{13} \tilde{x}_{\overline{2} 1}^{-1} \bar{\theta}_{21}\right)^{2} \rho_{14} \theta_{43} \tilde{x}_{\overline{4} 3}^{-1} \bar{\theta}_{24}\right. \\
& -\left(\theta_{13} \tilde{x}_{\overline{2} 1}^{-1} \bar{\theta}_{21}\right)^{2}\left(\theta_{43} \tilde{x}_{\overline{4} 3}^{-1} \bar{\theta}_{24}\right)^{2} \\
& \left.-2 \theta_{13} \tilde{x}_{\overline{2} 1}^{-1} \bar{\theta}_{21} \rho_{14}\left(\theta_{43} \tilde{x}_{\overline{4} 3}^{-1} \bar{\theta}_{24}\right) \theta_{43} \tilde{x}_{\overline{4} 3}^{-1} \tilde{x}_{\overline{2} 3} \tilde{x}_{\overline{2} 1}^{-1} \bar{\theta}_{21}\right] \\
= & 0 .
\end{aligned}
$$

We continue on this path and find two more equations of lowest order $(\theta \bar{\theta})^{4}$ :

$$
\begin{aligned}
T_{212}{ }^{3} \theta_{13} \tilde{x}_{\overline{2} 1}^{-1} \bar{\theta}_{21}= & 3\left[\theta_{13} \tilde{x}_{\overline{2} 1}^{-1} \bar{\theta}_{21} \rho_{14}^{2} \theta_{43} \tilde{x}_{\overline{4}}^{-1} \tilde{x}_{\overline{2} 3} \tilde{x}_{\overline{2} 1}^{-1} \bar{\theta}_{21}-\left(\theta_{13} \tilde{x}_{\overline{21}}^{-1} \bar{\theta}_{21}\right)^{2} \rho_{14}^{2}\right. \\
& -\theta_{13} \tilde{x}_{\overline{2} 1}^{-1} \bar{\theta}_{21} \rho_{14}^{2} \theta_{43} \tilde{x}_{\overline{4} 3}^{-1} \bar{\theta}_{24} \\
& \left.+\left(\theta_{13} \tilde{x}_{\overline{2} 1}^{-1} \bar{\theta}_{21}\right)^{2} \rho_{14} \theta_{43} \tilde{x}_{\overline{4} 3}^{-1} \bar{\theta}_{24}\right] \\
= & 0
\end{aligned}
$$




$$
\begin{aligned}
T_{212}{ }^{3} \theta_{43} \tilde{x}_{\overline{4} 3}^{-1} \tilde{x}_{\overline{2} 3} \tilde{x}_{\overline{2} 1}^{-1} \bar{\theta}_{21}= & 3\left[\theta_{43} \tilde{x}_{\overline{4} 3}^{-1} \bar{\theta}_{24} \rho_{14}^{2} \theta_{43} \tilde{x}_{\overline{4} 3}^{-1} \tilde{x}_{\overline{2} 3} \tilde{x}_{\overline{2} 1}^{-1} \bar{\theta}_{21}-\left(\theta_{43} \tilde{x}_{\overline{4} 3}^{-1} \bar{\theta}_{24}\right)^{2} \rho_{14}{ }^{2}\right. \\
& -\theta_{13} \tilde{x}_{\overline{2} 1}^{-1} \bar{\theta}_{21} \rho_{14}{ }^{2} \theta_{43} \tilde{x}_{\overline{4}}^{-1} \bar{\theta}_{24} \\
& \left.+\theta_{13} \tilde{x}_{\overline{2} 1}^{-1} \bar{\theta}_{21} \rho_{14}\left(\theta_{43} \tilde{x}_{\overline{4} 3}^{-1} \bar{\theta}_{24}\right)^{2}\right] \\
= & 0 .
\end{aligned}
$$

The last possible product of this kind, $T_{212}{ }^{3} \theta_{43} \tilde{x}_{\overline{4} 3}^{-1} \bar{\theta}_{24}$, is already zero with these conditions. At last the third power is still a long expression, but also the last to get to the final form of $T_{212}$ :

$$
\begin{aligned}
T_{212}{ }^{3}= & 3 \rho_{14}^{2} \theta_{43} \tilde{x}_{\overline{4} 3}^{-1} \tilde{x}_{\overline{2} 3} \tilde{x}_{\overline{2} 1}^{-1} \bar{\theta}_{21}-3 \rho_{14}^{2} \theta_{43} \tilde{x}_{\overline{4} 3}^{-1} \bar{\theta}_{24}-3 \rho_{14}{ }^{2} \theta_{13} \tilde{x}_{\overline{2} 1}^{-1} \bar{\theta}_{21} \\
& +3 \rho_{14}\left(\theta_{43} \tilde{x}_{\overline{4} 3}^{-1} \tilde{x}_{\overline{2} 3} \tilde{x}_{\overline{2} 1}^{-1} \bar{\theta}_{21}\right)^{2}-6 \rho_{14} \theta_{43} \tilde{x}_{\overline{4} 3}^{-1} \tilde{x}_{\overline{2} 3} \tilde{x}_{\overline{2} 1}^{-1} \bar{\theta}_{21} \theta_{43} \tilde{x}_{\overline{4} 3}^{-1} \bar{\theta}_{24} \\
& -6 \rho_{14} \theta_{43} \tilde{x}_{\overline{4} 3}^{-1} \tilde{x}_{\overline{2} 3} \tilde{x}_{\overline{2} 1}^{-1} \bar{\theta}_{21} \theta_{13} \tilde{x}_{\overline{2} 1}^{-1} \bar{\theta}_{21}+3 \rho_{14}\left(\theta_{43} \tilde{x}_{\overline{4} 3}^{-1} \bar{\theta}_{24}\right)^{2} \\
& +6 \rho_{14} \theta_{43} \tilde{x}_{\overline{4} 3}^{-1} \bar{\theta}_{24} \theta_{13} \tilde{x}_{\overline{2} 1}^{-1} \bar{\theta}_{21}+3 \rho_{14}\left(\theta_{13} \tilde{x}_{\overline{2} 1}^{-1} \bar{\theta}_{21}\right)^{2}-3 \theta_{43} \tilde{x}_{\overline{4} 3}^{-1} \bar{\theta}_{24}\left(\theta_{13} \tilde{x}_{\overline{2} 1}^{-1} \bar{\theta}_{21}\right)^{2} \\
& +6 \theta_{43} \tilde{x}_{\overline{4} 3}^{-1} \tilde{x}_{\overline{2} 3} \tilde{x}_{\overline{2} 1}^{-1} \bar{\theta}_{21} \theta_{43} \tilde{x}_{\overline{4} 3}^{-1} \bar{\theta}_{24} \theta_{13} \tilde{x}_{\overline{2} 1}^{-1} \bar{\theta}_{21}-3\left(\theta_{43} \tilde{x}_{\overline{4} 3}^{-1} \bar{\theta}_{24}\right)^{2} \theta_{13} \tilde{x}_{\overline{2} 1}^{-1} \bar{\theta}_{21} \\
& +12 \mathrm{i}\left[\rho_{14}{ }^{2} \theta_{43} \tilde{x}_{\overline{4} 3}^{-1} \bar{\theta}_{24} \theta_{13} \tilde{x}_{\overline{2} 1}^{-1} \bar{\theta}_{21}-\rho_{14}\left(\theta_{43} \tilde{x}_{\overline{4} 3}^{-1} \bar{\theta}_{24}\right)^{2} \theta_{13} \tilde{x}_{\overline{2} 1}^{-1} \bar{\theta}_{21}\right. \\
& \left.-\rho_{14} \theta_{43} \tilde{x}_{\overline{4} 3}^{-1} \bar{\theta}_{24}\left(\theta_{13} \tilde{x}_{\overline{2} 1}^{-1} \bar{\theta}_{21}\right)^{2}\right] \\
= & 0 .
\end{aligned}
$$

With all these conditions we finally find

$$
\begin{aligned}
T_{212}= & \rho_{14}-\theta_{43} \tilde{x}_{\overline{4} 3}^{-1} \bar{\theta}_{24}-\theta_{13} \tilde{x}_{\overline{2} 1}^{-1} \bar{\theta}_{21}+\theta_{43} \tilde{x}_{\overline{4} 3}^{-1} \tilde{x}_{\overline{2} 3} \tilde{x}_{\overline{2} 1}^{-1} \bar{\theta}_{21} \\
& -2 \mathrm{i}\left[\rho_{14}^{2}+\left(\theta_{43} \tilde{x}_{\overline{4} 3}^{-1} \bar{\theta}_{24}\right)^{2}+\left(\theta_{13} \tilde{x}_{\overline{2} 1}^{-1} \bar{\theta}_{21}\right)^{2}+\left(\theta_{43} \tilde{x}_{\overline{4} 3}^{-1} \tilde{x}_{\overline{2} 3} \tilde{x}_{\overline{2} 1}^{-1} \bar{\theta}_{21}\right)^{2}\right. \\
& \left.-2\left(\theta_{43} \tilde{x}_{\overline{4} 3}^{-1} \bar{\theta}_{24}+\theta_{13} \tilde{x}_{\overline{2} 1}^{-1} \bar{\theta}_{21}\right) \theta_{43} \tilde{x}_{\overline{4} 3}^{-1} \tilde{x}_{\overline{2} 3} \tilde{x}_{\overline{2} 1}^{-1} \bar{\theta}_{21}\right] \\
& +16 \theta_{43} \tilde{x}_{\overline{4} 3}^{-1} \tilde{x}_{\overline{2} 3} \tilde{x}_{\overline{2} 1}^{-1} \bar{\theta}_{21} \theta_{13} \tilde{x}_{\overline{2} 1}^{-1} \bar{\theta}_{21} \theta_{43} \tilde{x}_{\overline{4} 3}^{-1} \bar{\theta}_{24} .
\end{aligned}
$$

The four invariants introduced in this section can replace $I_{111}, I_{0}, I_{222}$ and $I_{212}$, whenever it seems convenient. This may take place because of their simpler structure and especially because of their derivatives, as we will see in section 5.3. Also we have already seen in the last section, that the three invariants $I_{111}, I_{0}$ and $I_{222}$ obviously are connected to invariant ratios of superconformal intervals, which we expanded in these invariants in eqns. 4.3.133-4.3.142 and several scattered other examples. Because the corresponding three invariants $T_{111}, T_{0}$ and $T_{222}$ are calculated from only these three nilpotent invariants, they are connected in the same way to these ratios. 


\section{Chapter 5}

\section{Correlation functions}

Correlation functions of observables given in form of fields are the standard results of theoretical calculations in quantum field theory, which can be compared to experimental data, e.g. the scattering amplitudes with results from high energy collider experiments. These scattering amplitudes in the S-matrix are calculated from time-ordered correlation functions with the LSZ-formula, which goes back to the publication of H. Lehmann, K. Symanzik, and W. Zimmerman, [LSZ55].

A perturbation series gives an empirically successful approximation for a small interaction. For strong couplings one can either look at lattice approximation, which are limited due to available computer power, or one has to find a way to get exact - because of the rampant perturbative approach often called non-perturbative - results. In the perturbation theory in quantum field theory there are mathematical problems with the appearing entities: The definition of non-linear functions of distributions is not clarified, the convergence of the perturbation series remains an open question and the used interaction picture conflicts Haag's theorem. Haag's theorem ${ }^{1}$ states, that the interaction picture is inconsistent with basic properties of interacting relativistic quantum field theories, such as translation invariance. It remains open, why the mainstream approach leads to empirically correct results despite the ignorance of Haag's theorem.

The standard model of particle physics consists of gauge theories. Although the Wightman approach cannot be directly used for gauge theories because of the absence of a Hilbert space in the mathematical sense - the norm is indefinite -, the situation is better in Coulomb gauge as recognized in the context of the ChristLee model and the Gribov-Zwanziger approach, [CL80, Zwa81, BZ81]. Within the Wightman approach the Wightman reconstruction theorem shows, that the correlation functions contain all information to recover the whole theory. This means, that from all n-point Wightman functions satisfying the Wightman axioms the existence of a separable Hilbert space with a vacuum vector and a field with a domain dense in the Hilbert space and containing the vacuum vector can be found and is unique up to unitary equivalence.

\footnotetext{
${ }^{1}$ Haag's theorem has been formulated first in Haa55]. A historical review on versions, proofs and reception can be found in EF06.
} 
Symmetries provide the most direct possibility to learn something about the nonperturbative structure of correlation functions. In this thesis we study Wightman functions, which are correlation functions without time-ordering ${ }^{2}$ as defined within the Wightman axiomatic approach.

In the main part of this chapter we investigate the implications of $\mathcal{N}=1$ superconformal symmetry, as given by representations of the four-fold cover $S U(2,2 \mid 1)$, on chiral scalar three- and four-point functions. In this case anomalous dimensions appear in the theory in contrast to the case, when we deal with global superconformal symmetry. The latter has only representations directly on Minkowski space or on superspace, if we express fields of a multiplet on Minkowski space within one superfield, and do not have representations on a larger covering space. In section 5.3 .4 we see the special properties of the - now rational - four point functions in this case.

This prepares the study of the positive energy condition for the chiral scalar four-point function in these global superconformal theories. It enables us to directly transfer the partial wave expansion for scalar conformal four-point functions to their supersymmetric pendant in section 6.2 .

Only the consequences of the superconformal symmetry and the restrictions of the superfields are discussed here without any reference to a specific model.

First of all we look at the general transformation properties of superconformal correlation functions. While the superfields transform covariantly under superconformal transformations, as seen in (3.4.91), the correlation function stays invariant:

$$
\left\langle\Psi_{1}^{\prime \xi_{1}}\left(z_{1}\right) \ldots \Psi_{n}^{\prime \xi_{n}}\left(z_{n}\right)\right\rangle=\left\langle\Psi_{1}^{\xi_{1}}\left(z_{1}\right) \ldots \Psi_{n}^{\xi_{n}}\left(z_{n}\right)\right\rangle
$$

One can plug in the superfield transformations here. This leads to a factor directly determined by these transformation properties times a function with the following properties (cf. Par99]). This function transforms homogeneously and is in general a function of $n-2$ variables. In the case of vanishing total $\mathrm{R}$-charge of the correlation function, which is the sum of the R-charges of the superfields therein, it is an invariant function.

Non-vanishing correlation functions of the component fields always have a vanishing total R-charge. So the total R-charge of a correlation function of superfields has to vanish for it to be non-nilpotent. For non-vanishing total R-charge and thus nilpotent correlation functions of scalar chiral superfields, the Ward identities seem to be rather restrictive: It was shown in [Osb99], that the total R-charge of the three point function has to be 1 .

Here the nilpotent case will only be touched in the repective ends of the discussions of the two and the three point function. So in rest of the chapter we have for the R-charges $\kappa_{i}$ of the superfields in a $n$-point function

$$
\sum_{i=1}^{n} \kappa_{i}=0 .
$$

\footnotetext{
${ }^{2}$ The time-ordering is needed in the perturbative approach to handle integrations over the whole space-time of inserted powers of the interaction term.
} 
In general the scalar $n$-point function in superconformal field theory is a function of all invariants of $n$ points times a factor due to the superconformal transformations of the $n$ fields (cf. Par99]):

$$
\left\langle S_{1} \ldots S_{n}\right\rangle=\frac{F(\text { n-point invariants })}{\prod_{l, m=1 ; l \neq m}^{n} x_{\overline{l m}}^{2}{ }^{\Delta_{l m}}},
$$

where

$$
\Delta_{l m}=-\frac{1}{2(n-1)(n-2)} \sum_{i=1}^{n} \eta_{i}+\frac{1}{2(n-2)}\left(\eta_{l}+\eta_{m}\right)+\frac{3}{2 n}\left(\kappa_{l}-\kappa_{m}\right) .
$$

Here we are interested in chiral scalar superfields. As these depend only on the chiral variables, one immediately sees, that there is no way to construct a three point invariant with only half the Graßmann variables. Therefore the three point function can be easily written down in the next section, as it was already done in e.g. Osb99].

A four point function with vanishing total R-charge depends on two chiral and two anti-chiral variables (here $\left(x_{1-}, \bar{\theta}_{1}\right),\left(x_{2-}, \bar{\theta}_{2}\right),\left(x_{3+}, \bar{\theta}_{3}\right)$ and $\left.\left(x_{4+}, \bar{\theta}_{4}\right)\right)$, as will be seen in section 5.3 . This leads to the problem, that there is only one superconformal cross ratio given by eq. (4.2.2) depending only on these four variables, namely $\mathcal{I}_{2}$. But there have to be two independent non-nilpotent superconformal four-point invariants, because there are two conformal four-point invariants, on which the fourpoint functions of the component fields depend. One possibility is the construction of a trace invariant as the second non-nilpotent invariant (cf. [Osb99]).

Here two superconformal cross ratios, $\mathcal{I}_{1}$ and $\mathcal{I}_{2}$, and the set of nilpotent invariants $\mathfrak{I}$ from eq. 4.3 .40 will be used to get an expression for the four-point functions. This will simplify conclusions from properties of global conformal field theories to global superconformal field theories.

With the help of the chirality conditions applied to the four-point function, the dependence on all these invariants can be reduced to a dependence only on two cross ratios with a fixed universal differential operator applied to the rest of the resulting expression of the correlation function, which is essentially the four-point function of the lowest order component fields of the superfields and contains the model specific information.

For rational four-point functions this differential operator can be applied to their power series. The coefficients of this power series turn out to be the same as those of the conformal four-point function of the scalar fields, which are the lowest components of the chiral and anti-chiral fields.

\subsection{The two-point function}

Here we recall the superconformal scalar chiral two point function with vanishing $\mathrm{R}$-charge. Because of the equal absolute value of the R-charge of the two scalar fields they have to have the same scaling dimension, $\eta$. We have seen, that there are no two-point invariants. So the two-point function is up to a constant completely 
determined by the superconformal transformations of the two scalar superfields, just as for all other superfields. If we plug the superfield transformations into eq. (5.0.1) we get

$$
\left\langle\bar{\Phi}_{1}^{\prime}\left(z_{1-}\right) \Phi_{2}^{\prime}\left(z_{2+}\right)\right\rangle=\Omega_{-}\left(z_{1-}, g\right)^{\eta} \Omega_{+}\left(z_{2+}, g\right)^{\eta}\left\langle\bar{\Phi}_{1}^{\prime}\left(z_{1-}^{\prime}\right) \Phi_{2}^{\prime}\left(z_{2+}^{\prime}\right)\right\rangle .
$$

We have already found the functions on superspace with this transformation property in eq. (4.2.1). There can only be one. If there were two, the quotient of both would be an invariant of two points, which does not exist. So we end up with

$$
\left\langle\bar{\Phi}_{1}\left(x_{1-}^{\mu}, \bar{\theta}_{1}\right) \Phi_{2}\left(x_{2+}^{\mu}, \theta_{2}\right)\right\rangle=C \frac{1}{\left(x_{\overline{1} 2}^{2}\right)^{\eta}} .
$$

From (3.2.18) one easily verifies the conditions on the two-point function given by the restrictions (3.4.75) and (3.4.76),

$$
\left\langle D_{1-} \bar{\Phi}_{1} \Phi_{2}\right\rangle=0 \quad\left\langle\Phi_{1}^{+} \bar{D}_{2+} \Phi_{2}\right\rangle=0 .
$$

R-symmetry violating two point function A very special two point function of two chiral superfields is given in Osb99. Only if the sum of the R-charges of the two superfields is three, it does not vanish. In this case it is given by a pure contact term:

$$
\left\langle\Phi_{1}\left(z_{1+}\right) \Phi_{2}\left(z_{2+}\right)\right\rangle=C \delta^{4}\left(x_{1+}-x_{2+}\right) \theta_{12}^{2}, \quad \eta_{1}+\eta_{2}=3 .
$$

This two-point function conflicts with the Wightman axioms, as it violates the spectral condition given in section 2.1 .

\subsection{The three-point function}

The three-point function is also determined up to a constant just like the ordinary conformal three-point function. But in contrast to the two-point function this is due to the restrictions of the specific type of fields. The three point function with vanishing $\mathrm{R}$-charge discussed here either contain one or two chiral superfields and correspondingly two or one anti-chiral superfields. Here we look at the latter case keeping in mind, that the former case calculates analoguesly. From eq. (5.0.1) we get the following transformation properties for the chiral scalar three point function:

$$
\begin{aligned}
\left\langle\bar{\Phi}_{1}^{\prime}\left(z_{1-}\right) \Phi_{2}^{\prime}\left(z_{2+}\right) \Phi_{3}^{\prime}\left(z_{3+}\right)\right\rangle= & \Omega_{-}\left(z_{1-}, g\right)^{\eta_{1}} \Omega_{+}\left(z_{2+}, g\right)^{\eta_{2}} \Omega_{+}\left(z_{3+}, g\right)^{\eta_{3}} \\
& \left\langle\bar{\Phi}_{1}^{\prime}\left(z_{1-}^{\prime}\right) \Phi_{2}^{\prime}\left(z_{2+}^{\prime}\right) \Phi_{3}^{\prime}\left(z_{3+}^{\prime}\right)\right\rangle
\end{aligned}
$$

with $\eta_{1}=\eta_{2}+\eta_{3}$. This is fulfilled by the function

$$
\left\langle\bar{\Phi}_{1}\left(x_{1-}^{\mu}, \bar{\theta}_{1}\right) \Phi_{2}\left(x_{2+}^{\mu}, \theta_{2}\right) \Phi_{3}\left(x_{3+}^{\mu}, \theta_{3}\right)\right\rangle=\frac{f_{3 P F}\left(I_{3 P F}\right)}{x_{\overline{1} 2}^{2 \eta_{2}} x_{\overline{1} 3}^{2 \eta_{3}}},
$$

The denominator totally accounts for the factors from the transformation and leaves the function $f_{3 P F}$ of the superconformal invariant. 
However, also here the chiral and anti-chiral constraints of the chiral and antichiral superfields have to be satisfied. Eqns. (3.4.75) and (3.4.76) have to hold for the fields in the correlation functions and so the derivatives of the correlation functions have to vanish.

$$
D_{1-}\left\langle\bar{\Phi}_{1} \Phi_{2} \Phi_{3}\right\rangle=0, \quad \bar{D}_{2+}\left\langle\bar{\Phi}_{1} \Phi_{2} \Phi_{3}\right\rangle=0, \quad \bar{D}_{3+}\left\langle\bar{\Phi}_{1} \Phi_{2} \Phi_{3}\right\rangle=0,
$$

If we now choose the restriction of the chiral field $\Phi_{2}\left(z_{2+}\right)$ to be examined further we only have to derive the invariant $I_{3 P F}$ and get two summands:

$$
\bar{D}_{2+} I_{3 P F}=-\frac{\partial}{\partial \bar{\theta}_{2}}\left(\frac{x_{\overline{2} 3}^{2} x_{\overline{3} 1}^{2} x_{\overline{1} 2}^{2}}{x_{\overline{3} 2}^{2} x_{\overline{1} 3}^{2} x_{\overline{2} 1}^{2}}-1\right)=4 \mathrm{i} \frac{x_{\overline{2} 3}^{2} x_{\overline{3} 1}^{2} x_{\overline{1} 2}^{2}}{x_{\overline{3} 2}^{2} x_{\overline{1} 3}^{2} x_{\overline{2} 1}^{2}}\left(\theta_{23} \tilde{x}_{\overline{2} 3}^{-1}-\theta_{21} \tilde{x}_{\overline{2} 1}^{-1}\right) .
$$

We expand the function $f_{3 P F}$ in a Taylor series, which terminates because the third power of $I_{3 P F}$ is zero:

$$
f_{3 P F}\left(I_{3 P F}\right)=f_{(3 P F, 0)}+f_{(3 P F, 1)} I_{3 P F}+f_{(3 P F, 2)} I_{3 P F}^{2} .
$$

We can now look at the lowest order $\bar{\theta}$, which has to vanish independently.

$$
\bar{D}_{2+}\left\langle\bar{\Phi}_{1}^{\prime}\left(z_{1-}\right) \Phi_{2}^{\prime}\left(z_{2+}\right) \Phi_{3}^{\prime}\left(z_{3+}\right)\right\rangle \stackrel{\text { l.o. }}{=} 4 \mathrm{i} \frac{f_{(3 P F, 1)} I_{3 P F}}{x_{12}^{2 \eta_{2}} x_{13}^{2 \eta_{3}}}\left(\theta_{23} \tilde{x}_{23}^{-1}-\theta_{21} \tilde{x}_{21}^{-1}\right)
$$

Obviously only $f_{3 P F, 1}=0$ can accomplish this. But this also leaves no possibilities to cancel terms of the derivative of $I_{3 P F}^{2}$ in the next order, so that also $f_{3 P F, 2}=0$. Already with only one of the three restrictions from eq. (5.2.3) the chiral/anti-chiral three point function is fixed up to a constant, $C=f_{(3 P F, 0)}$, and has the known form:

$$
\left\langle\bar{\Phi}_{1}\left(x_{1-}^{\mu}, \bar{\theta}_{1}\right) \Phi_{2}\left(x_{2+}^{\mu}, \theta_{2}\right) \Phi_{3}\left(x_{3+}^{\mu}, \theta_{3}\right)\right\rangle=\frac{C}{x_{\overline{1} 2}^{2 \eta_{2}} x_{\overline{1} 3}^{2 \eta_{3}}} .
$$

In the same way one finds the expression for two anti-chiral and one chiral superfield:

$$
\left\langle\bar{\Phi}_{1}\left(x_{1-}^{\mu}, \bar{\theta}_{1}\right) \bar{\Phi}_{2}\left(x_{2-}^{\mu}, \bar{\theta}_{2}\right) \Phi_{3}\left(x_{3+}^{\mu}, \theta_{3}\right)\right\rangle=\frac{C}{x_{\overline{1} 3}^{2 \eta_{1}} x_{\overline{2} 3}^{2 \eta_{2}}} .
$$

with $\eta_{3}=\eta_{1}+\eta_{2}$.

In eq. (5.0.3) we have seen Park's general form of scalar n-point functions. Here we want to see in the following short calculation, how the chiral three point function fits into this form, which is

$$
\left\langle\bar{\Phi}_{1}\left(x_{1-}^{\mu}, \bar{\theta}_{1}\right) \Phi_{2}\left(x_{2+}^{\mu}, \theta_{2}\right) \Phi_{3}\left(x_{3+}^{\mu}, \theta_{3}\right)\right\rangle=\frac{f_{\text {Park }}\left(I_{3 P F}\right)}{\prod_{l, m=1 ; l \neq m}^{3} x_{\overline{l m}^{2}}^{2} \Delta_{l m}} .
$$

It contains an arbitrary function of the three-point invariant, $I_{3 P F}$. Again the restrictions (3.4.75) and (3.4.76) and so the differential equations, have to be satisfied. 
But it is easier to rewrite the denominator with the help of the identities relating the $\Delta_{l m}$ with each other:

$$
\begin{aligned}
& \Delta_{12}+\Delta_{13}=\eta_{1}, \\
& \Delta_{12}+\Delta_{32}=\eta_{2}, \\
& \Delta_{13}+\Delta_{23}=\eta_{3}, \\
& \Delta_{21}+\Delta_{31}=\Delta_{21}+\Delta_{23}=\Delta_{31}+\Delta_{32}=0 .
\end{aligned}
$$

Here we used eq. 5.0 .4 with $n=3$, eqns. 3.4.59 and 3.4.60 with the corresponding signs. With these identities we get

$$
\frac{1}{\prod_{l, m=1 ; l \neq m}^{3} x_{\overline{l m}}^{2}{ }^{\Delta_{l m}}}=\frac{J_{1} \Delta_{21}}{x_{\overline{1} 2}^{2 \eta_{2}} x_{\overline{1} 3}^{2 \eta_{3}}} .
$$

The derivatives only vanish, if the whole does not depend on $I_{3 P F}$ any more. So with eq. 4.1.8 the function $f_{\text {Park }}\left(I_{3 P F}\right)$ is

$$
f_{\text {Park }}\left(I_{3 P F}\right)=\left(I_{3 P F}+1\right)^{-\Delta_{21}} .
$$

And also here the calculations are analogous, which lead to the function, which is needed to fit the three point function of two anti-chiral and one chiral field into the form of Park. Due to the different sign for the index 2, when we use eq. (3.4.60) instead of eq. (3.4.59), the identities for $\Delta_{l m}$ change correspondingly and we get again the same function in the numerator, although the denominator in $(5.2 .8)$ is different:

$$
\left\langle\bar{\Phi}_{1}\left(x_{1-}^{\mu}, \bar{\theta}_{1}\right) \bar{\Phi}_{2}\left(x_{2-}^{\mu}, \bar{\theta}_{2}\right) \Phi_{3}\left(x_{3+}^{\mu}, \theta_{3}\right)\right\rangle=\frac{\left(I_{3 P F}+1\right)^{-\Delta_{21}}}{\prod_{l, m=1 ; l \neq m}^{3} x_{\overline{l m}_{m}}^{\Delta_{l m}}} .
$$

This recapitulation of two- and three-point functions in the light of the chiral and anti-chiral restrictions has shown, how exactly analogous results to the ordinary conformal case come out within supersymmetry.

R-symmetry violating three point function In eq. 4.1.19 we listed the only two three point invariants of superconformal symmetry without R-symmetry. They have an R-charge of 1 and -1 , respectively. With these there is the special case of two three point functions of three anti-chiral superfield respectively three chiral superfields, which violate R-symmetry and have the same total R-charges as these invariants. Thus the sums of the scaling dimensions of the three anti-chiral superfields, as well as the three chiral superfields has to be three. They were given in [Osb99] and we write them down here pointing out the invariant in it:

$$
\begin{aligned}
\left\langle\bar{\Phi}_{1}\left(x_{1-}^{\mu}, \bar{\theta}_{1}\right) \bar{\Phi}_{2}\left(x_{2-}^{\mu}, \bar{\theta}_{2}\right) \bar{\Phi}_{3}\left(x_{3-}^{\mu}, \bar{\theta}_{3}\right)\right\rangle & =\frac{C}{x_{\overline{2} 1}^{2 \eta_{2}} x_{\overline{3} 1}^{2 \eta_{3}}} X_{1+}^{2\left(\eta_{1}-\frac{3}{2}\right)} \frac{\Theta_{1} \tilde{\Theta}_{1}}{\sqrt{X_{1+}^{2}}} \\
\left\langle\Phi_{1}\left(x_{1+}^{\mu}, \theta_{1}\right) \Phi_{2}\left(x_{2+}^{\mu}, \theta_{2}\right) \Phi_{3}\left(x_{3+}^{\mu}, \theta_{3}\right)\right\rangle & =\frac{C}{x_{\overline{1} 2}^{2 \eta_{2}} x_{\overline{1} 3}{ }^{2 \eta_{3}}} X_{1+}^{2\left(\eta_{1}-\frac{3}{2}\right)} \frac{\tilde{\Theta}_{1} \bar{\Theta}_{1}}{\sqrt{X_{1+}^{2}}}
\end{aligned}
$$


with $\eta_{1}+\eta_{2}+\eta_{3}=3$ in both cases.

We now pick the latter to demonstrate, that the right hand side has the correct transformation properties, while the form of three point function of only anti-chiral superfields can be shown analogously.

With eqns. 4.2.1), 4.2.15), 4.2.17) and 4.1.12) we find, that the right hand side transforms as

$$
\begin{aligned}
\left(\frac{X_{1+}^{2\left(\eta_{1}-\frac{3}{2}\right)}}{x_{\overline{1} 2}^{2 \eta_{2}} x_{\overline{1} 3}^{2 \eta_{3}}} \frac{\tilde{\Theta}_{1} \bar{\Theta}_{1}}{\sqrt{X_{1+}^{2}}}\right)^{\prime}= & \Upsilon_{1}\left(z_{1}\right) \Omega_{-}\left(z_{1-}, g\right)^{-\eta_{2}-\eta_{3}-\left(\eta_{1}-\frac{3}{2}\right)} \Omega_{+}\left(z_{1+}, g\right)^{-\left(\eta_{1}-\frac{3}{2}\right)} \\
& \Omega_{+}\left(z_{2+}, g\right)^{-\eta_{2}} \Omega_{+}\left(z_{3+}, g\right)^{-\eta_{3}}\left(\frac{X_{1+}^{2\left(\eta_{1}-\frac{3}{2}\right)}}{x_{\overline{1} 2}{ }^{2 \eta_{2}} x_{\overline{13}}{ }^{2 \eta_{3}}} \frac{\tilde{\Theta}_{1} \bar{\Theta}_{1}}{\sqrt{X_{1+}^{2}}}\right) .
\end{aligned}
$$

This can be simplified with the condition, that the sum of the scale dimensions is three and eq. (3.4.95):

$$
\left(\frac{X_{1+}^{2\left(\eta_{1}-\frac{3}{2}\right)}}{x_{\overline{1} 2}^{2 \eta_{2}} x_{\overline{1} 3}^{2 \eta_{3}}} \frac{\tilde{\Theta}_{1} \bar{\Theta}_{1}}{\sqrt{X_{1+}^{2}}}\right)^{\prime}=\Omega_{+}\left(z_{1+}, g\right)^{-\eta_{1}} \Omega_{+}\left(z_{2+}, g\right)^{-\eta_{2}} \Omega_{+}\left(z_{3+}, g\right)^{-\eta_{3}}(\ldots)
$$

where the dots are the same as in the bracket above. This is exactly the transformation of the three point function of three chiral superfields. One can check, that the anti-chiral and chiral constraints of these three point function are satisfied. It can be relatively complicated to perform the derivatives directly in comparison to the alternative way permuting the indices. As these three point functions have to be invariant under such permutations for certain points in superspace, one can cyclically permute them in these cases and gets for each of the three indices an expression of the three point functions, in which the respective derivative is trivial. These are the derivatives with respect to $\theta_{i}$ for the three point function in eq. (5.2.17) and with respect to $\bar{\theta}_{i}$ for the three point function in eq. (5.2.18). A similar argument is already given in the publication Osb99], while he also uses the properties caused by the nilpotency of $\Theta_{1}$ and $\overline{\Theta_{1}}$.

In a following publication DO01a about correlation functions of only chiral superfields only infinitesimal transformations of a smaller symmetry group are considered. These correlation functions can be written down by only chiral variables, so that no cancellations of terms of anti-chiral variables are needed.

\subsection{The four-point function}

In this section we express the four-point function in terms of the set of four-point invariants, which we have chosen in the sections 4.2 .1 and 4.3 . These seem especially promising in order to be able to trace properties of this four-point function back to properties of conformal scalar four-point functions. This expectation is based on 
the fact, that the two superconformal cross ratios reduce to conformal cross ratios and all nilpotent invariants vanish, if the Graßmann variables are set to zero. The coefficients belonging to the superconformal cross ratios in an expansion of a rational four-point function, as we have them in section 5.3.4, are not changed in this step and no change in the summations are needed, as it would be, if we used other non-nilpotent invariants, which are no cross ratios.

First of all the general form of the scalar four-point function given by eq. (5.0.3) for $n=4$ can be rewritten with the help of $\mathcal{I}_{1}, \mathcal{I}_{2}, J_{1}, J_{1(1)}$ and $J_{1(2)}$ :

$$
\begin{aligned}
\left\langle S_{1} \ldots S_{4}\right\rangle= & \mathcal{I}_{1}{ }^{\Lambda_{34}} \mathcal{I}_{2}{ }^{\Lambda_{24}} J_{1}{ }^{\Delta_{43}-\Delta_{31}} J_{1(1)}{ }^{-\Delta_{43}-\Delta_{41}} J_{1(2)}{ }^{\Delta_{43}}\left(\frac{x_{\overline{1} 2}{ }^{2}}{x_{\overline{2} 1}{ }^{2}}\right)^{\Sigma_{1}} \\
& \left(\frac{x_{\overline{2} 3}{ }^{2}}{x_{\overline{3} 2}{ }^{2}}\right)^{\Sigma_{2}}\left(\frac{x_{\overline{2} 4}{ }^{2}}{x_{\overline{4} 2}{ }^{2}}\right)^{\Sigma_{3}}\left(\frac{x_{\overline{2} 3}{ }^{2}}{x_{\overline{1} 2}{ }^{2} x_{\overline{1} 3}{ }^{2}}\right)^{\Xi} \frac{F\left(\mathcal{I}_{1}, \mathcal{I}_{2}, I_{111}, \ldots, I_{222}\right)}{x_{\overline{1} 3}{ }^{2\left(\eta_{3}-\eta_{2}\right)} x_{\overline{1} 4}{ }^{2 \eta_{4}} x_{\overline{2} 3}{ }^{2 \eta_{2}}}
\end{aligned}
$$

with

$$
\begin{aligned}
\Lambda_{l m} & =\Delta_{l m}+\Delta_{m l}=-\frac{1}{6} \sum_{i=1}^{4} \eta_{i}+\frac{1}{2}\left(\eta_{l}+\eta_{m}\right), \\
\Sigma_{1} & =\Delta_{21}+\Delta_{31}+\Delta_{41}=\frac{1}{2} \eta_{1}-\frac{3}{2} \kappa_{1}, \\
\Sigma_{2} & =\Delta_{31}+\Delta_{32}+\Delta_{34}=\frac{1}{2} \eta_{3}-\frac{3}{2} \kappa_{3}, \\
\Sigma_{3} & =\Delta_{41}+\Delta_{42}+\Delta_{43}=\frac{1}{2} \eta_{4}-\frac{3}{2} \kappa_{4}, \\
\Xi & =\frac{1}{2}\left(\eta_{1}+\eta_{2}-\eta_{3}-\eta_{4}\right),
\end{aligned}
$$

where eq. 5.0 .2 is used to calculate $\Sigma_{1}, \Sigma_{2}$ and $\Sigma_{3}$. Because the invariant prefactors pulled out can be expressed in terms of the invariants, which are the arguments of $F$, we can define a new function $f_{4 P F}$, so that

$$
\begin{aligned}
\left\langle S_{1} \ldots S_{4}\right\rangle= & \left(\frac{x_{\overline{1} 2}{ }^{2}}{x_{\overline{2} 1}{ }^{2}}\right)^{\Sigma_{1}}\left(\frac{x_{\overline{2} 3}{ }^{2}}{x_{\overline{3} 2}{ }^{2}}\right)^{\Sigma_{2}}\left(\frac{x_{\overline{2} 4}{ }^{2}}{x_{\overline{4} 2}{ }^{2}}\right)^{\Sigma_{3}}\left(\frac{x_{\overline{2} 3}{ }^{2}}{x_{\overline{1} 2}{ }^{2} x_{\overline{1} 3}{ }^{2}}\right)^{\Xi} \\
& \frac{f_{4 P F}\left(\mathcal{I}_{1}, \mathcal{I}_{2}, I_{111}, \ldots, I_{222}\right)}{x_{\overline{1} 3}{ }^{2\left(\eta_{3}-\eta_{2}\right)} x_{\overline{1} 4}{ }^{2 \eta_{4}} x_{\overline{2} 3}{ }^{2 \eta_{2}}}
\end{aligned}
$$

In general the function $f_{4 P F}$ has the expansion

$$
f_{4 P F}=\sum_{i=0}^{4} \sum_{j=1}^{n_{i}} f_{i, j}\left(\mathcal{I}_{1}, \mathcal{I}_{2}\right) \mathfrak{I}_{i, j}
$$

with respect to the nilpotent invariants 4.3 .40$)$, where the coefficients are functions of the two cross ratios. If the Graßmann variables are set to zero, the superconformal four-point function passes into the conformal four-point function. The cross ratios 
reduce to conformal cross ratios and all $\mathfrak{I}_{i, j}$ with $i>1$ vanish. Thus the function $f_{0,1}$ is the function appearing in the scalar four-point function of conformal field theory.

We want to see, what consequences the restrictive conditions defining chiral and anti-chiral superfields have on the scalar four-point functions, eq. (5.3.7). In order to get a general expression of the chiral scalar four-point functions with vanishing total R-charge, the following four differential equations have to be solved:

$$
\begin{array}{llrl}
D_{1-}\langle\bar{\Phi} \bar{\Phi} \Phi \Phi\rangle=0, & & D_{2-}\langle\bar{\Phi} \bar{\Phi} \Phi \Phi\rangle=0 \\
\bar{D}_{3+}\langle\bar{\Phi} \bar{\Phi} \Phi \Phi\rangle=0, & & \bar{D}_{4+}\langle\bar{\Phi} \bar{\Phi} \Phi \Phi\rangle=0 .
\end{array}
$$

The following theorem states the solution of the differential equations.

Theorem 5.1. All chiral/anti-chiral scalar four-point functions with vanishing total $R$-charge are of the form

$$
\left\langle\bar{\Phi}_{1}\left(z_{1-}\right) \bar{\Phi}_{2}\left(z_{2-}\right) \Phi_{3}\left(z_{3+}\right) \Phi_{4}\left(z_{4+}\right)\right\rangle=\mathcal{D} \frac{f_{0,1}\left(\mathcal{I}_{1}, \mathcal{I}_{2}\right)}{x_{\overline{1} 3}{ }^{2\left(\eta_{3}-\eta_{2}\right)} x_{\overline{1} 4^{2 \eta_{4}} x_{\overline{2} 3}{ }^{2 \eta_{2}}}}
$$

with the zeroth order coefficient, $f_{0,1}$, of the expansion of $f_{4 P F}$ with respect to the nilpotent invariants (eq. (5.3.8) ) and a fixed, universal differential operator

$$
\mathcal{D}=e^{4 \mathrm{i} T\left(\mathcal{I}_{1}, \mathcal{I}_{2}, I_{111}, \ldots, I_{222}\right) \mathcal{I}_{1} \frac{\partial}{\partial \mathcal{I}_{1}}} .
$$

The fixed function $T$ is nilpotent and will be given in the course of the proof in eq. (5.3.44). The exponential with the derivatives has to be understood in form of its Taylor expansion with only finitely many terms because of the nilpotency of $T$.

Proof. This proof goes over most of the rest of this section. To underline its structure the two important parts, into which it divides, each form their own subsection. The first gives results in lowest order, which are needed to start iterative steps completing the proof in the second subsection.

But first we have to rewrite the problem in a more appropriate form. The starting point is the general scalar four point function. The chiral/anti-chiral scalar four-point function has to be of the same form, eq. (5.3.7).

However, scale dimensions and R-charges of the chiral and anti-chiral superfields fulfill eqns. (3.4.59) and 3.4.60). Consequently $\Sigma_{1}=\Sigma_{2}=\Sigma_{3}=0$ (eqns. (5.3.3)(5.3.6) and with eq. 5.0 .2 also $\Xi=0$. Thus the four-point function reduces to

$$
\left\langle S_{1} \ldots S_{4}\right\rangle=\frac{f_{4 P F}\left(\mathcal{I}_{1}, \mathcal{I}_{2}, I_{111}, \ldots, I_{222}\right)}{x_{\overline{1} 3}{ }^{2\left(\eta_{3}-\eta_{2}\right)} x_{\overline{1}{ }^{2}{ }^{2 \eta_{4}} x_{\overline{2} 3}{ }^{2 \eta_{2}}}} .
$$

The derivatives in the differential equations (5.3.9) applied to the denominator of this form and to the superconformal cross ratio, $\mathcal{I}_{2}$ are zero. Thus for the differential equations to be satisfied the derivatives of the function $f_{4 P F}$ have to vanish. These derivatives can be evaluated using the expansion (5.3.8):

$$
\mathfrak{D} f_{4 P F}=\sum_{i=0}^{3} \sum_{j=1}^{n_{i}} \frac{\partial f_{i, j}}{\partial \mathcal{I}_{1}}\left(\mathfrak{D} \mathcal{I}_{1}\right) \mathfrak{I}_{i, j}+\sum_{k=1}^{4} \sum_{l=1}^{n_{k}} f_{k, l} \mathfrak{D I}_{k, l}
$$


with $\mathfrak{D} \in\left\{D_{1-}, D_{2-}, \bar{D}_{3+}, \bar{D}_{4+}\right\}$.

The sum over $i$ ends at $i=3$ because the product of $\mathfrak{I}_{4,1}$ and the derivative of $\mathcal{I}_{1}$ is zero due to their nilpotency. We now show, that this is the case.

The derivative of $\mathcal{I}_{1}$ either vanishes or contains a term, which is in lowest order proportional to the derivative of nilpotent functions of three points. We have the following derivatives:

$$
\begin{aligned}
D_{1-} \mathcal{I}_{1} & =\bar{D}_{4+} \mathcal{I}_{1}=0 \\
D_{2-} \mathcal{I}_{1} & =\mathcal{I}_{1}(-4 \mathrm{i})\left(\tilde{\mathrm{x}}_{\overline{3} 2}^{-1} \bar{\theta}_{32}-\tilde{\mathrm{x}}_{\overline{1} 2}^{-1} \bar{\theta}_{12}\right) \\
\bar{D}_{3+} \mathcal{I}_{1} & =\mathcal{I}_{1}(4 \mathrm{i})\left(\theta_{32} \tilde{\mathrm{x}}_{\overline{3} 2}^{-1}-\theta_{34} \tilde{\mathrm{x}}_{\overline{3} 4}^{-1}\right)
\end{aligned}
$$

We want to relate these expressions to lowest order of $\tilde{\bar{\Theta}}_{1(2)}$, $\tilde{\Theta}_{1}$ and $\tilde{\bar{\Theta}}_{1}$. The lowest order expressions of the nilpotent invariants can be found in appendix C.1. We can directly see from eq. C.1.13, that the derivative $D_{2-} I_{0}$ is proportional to the lowest order of the first expression:

$$
D_{2-} I_{0} \stackrel{\text { l.o. }}{=} \frac{x_{14}^{2} x_{23}^{2}}{x_{13}^{2} x_{24}^{2}}\left(\tilde{\mathrm{x}}_{12}^{-1} \bar{\theta}_{12}-\tilde{\mathrm{x}}_{32}^{-1} \bar{\theta}_{32}\right)
$$

where the antisymmetry of the lowest order intervals was used.

On the other hand this is also given by eq. (C.1.21) and so is proportional to $\tilde{\Theta}_{1}$. Together with eq. 4.2 .26 the product $I_{111}{ }^{2} I_{222}{ }^{2} D_{2}-\mathcal{I}_{1}$ thus can be given as a sum of one term of third power of $\tilde{\Theta}_{1(1)}$ and one of third power of $\tilde{\Theta}_{1(2)}$, so that it vanishes.

The lowest order of the other derivative, eq. (5.3.16), is proportional to a sum of two derivative of nilpotent invariants:

$$
\begin{aligned}
\bar{D}_{3+} I_{0}+\frac{x_{14}{ }^{2} x_{23}{ }^{2}}{x_{12}{ }^{2} x_{43}{ }^{2}} \bar{D}_{3+} I_{222} \stackrel{\text { l.o. }}{=} & \frac{x_{14}^{2} x_{23}^{2}}{x_{13}^{2} x_{24}{ }^{2}}\left(\theta_{32} \tilde{\mathrm{x}}_{32}^{-1}-\theta_{13} \tilde{\mathrm{x}}_{13}^{-1}\right) \\
& +\frac{x_{14}{ }^{2} x_{23}{ }^{2}}{x_{13}{ }^{2} x_{24}{ }^{2}}\left(\theta_{13} \tilde{\mathrm{x}}_{13}^{-1}-\theta_{34} \tilde{\mathrm{x}}_{34}^{-1}\right) .
\end{aligned}
$$

With the help of eqns. C.1.22, C.1.18 and 4.2.27) we find this to be a sum of three terms containing either $\tilde{\Theta}_{1(1)}$ or $\tilde{\Theta}_{1(2)}$. Hence the product of this derivatives and $I_{111}^{2} I_{222}^{2}$ also has to vanish.

\subsubsection{Lowest Graßmannian order}

The structure of eq. (5.3.13), which has to vanish, suggests to look at the lowest a priori non-vanishing order, $\theta$ resp. $\bar{\theta}$, because it clarifies the relation between the function $f_{0,1}$ and the functions $f_{1, l}$ : In this lowest order we have to have

$$
\frac{\partial f_{0,1}}{\partial \mathcal{I}_{1}}\left(\mathfrak{D I} \mathcal{I}_{1}\right)+\sum_{l=1}^{8} f_{1, l} \mathfrak{D} \mathfrak{I}_{1, l}=0
$$


So the problem breaks down to the cancellation of the derivatives of the fourpoint invariants in lowest order. With the help of the calculations above we will quickly find a term of the form of the second summand, which is the negative first summand. Then we show, that there are no sums $\sum_{l=1}^{8} f_{1, l} \mathfrak{D} \mathfrak{I}_{1, l}$, which vanish on their own.

We have to look closer on the proportionalities mentioned above around eqns. (5.3.15) and 5.3.17) and eqns. 5.3.16) and 5.3.18). The first pair of equations gives

$$
D_{2-} \mathcal{I}_{1} \stackrel{\text { l.o. }}{=} 4 \mathrm{i} \mathcal{I}_{1} \frac{x_{13}^{2} x_{24}^{2}}{x_{14}{ }^{2} x_{23}{ }^{2}} D_{2-} I_{0 \text { l.o. }}
$$

In lowest order $I_{0}$ (eq. 4.3.70) is a sum of invariants $I_{i j k}$ :

$$
I_{0} \stackrel{\text { l.o. }}{=} I_{111}-I_{112}-I_{121}+I_{122}-I_{211}+I_{212}+I_{221}-I_{222},
$$

The second pair leads us to

$$
\bar{D}_{3+} \mathcal{I}_{1} \stackrel{\text { l.o. }}{=} 4 \mathrm{i} \mathcal{I}_{1}\left(\frac{x_{13}^{2} x_{24}^{2}}{x_{14}{ }^{2} x_{23}{ }^{2}} \bar{D}_{3+} I_{0 \text { l.o. }}+\frac{x_{13}^{2} x_{24}{ }^{2}}{x_{12}{ }^{2} x_{43}{ }^{2}} \bar{D}_{3+} I_{222}\right) .
$$

The first summand here has the same factor as the right hand side in eq. (5.3.21), while the second summand has not appeared before because $D_{2-} I_{222}=0$. So we find as part of a special solution

$$
\sum_{l=1}^{8} f_{1, l} \Im_{1, l}=-4 \mathrm{i} \mathcal{I}_{1}\left(\mathcal{I}_{2}{ }^{-1} I_{0}+\mathcal{I}_{1} \mathcal{I}_{2}{ }^{-1} I_{222}\right) \frac{\partial f_{0,1}}{\partial \mathcal{I}_{1}}+\ldots
$$

Here the conformal cross ratios appearing in eqns. (5.3.21) and 5.3.23) are replaced by $\mathcal{I}_{1}$ and $\mathcal{I}_{2}{ }^{-1}$, as there is no difference in lowest order.

But this is not the complete special solution we search for, as it only solves two of four equations given by the condition (5.3.20). It is still necessary to look at the derivatives $D_{1-}$ and $\bar{D}_{4+}$ and see, if these derivatives of the terms found here cancel, or if additional terms are needed.

Starting with $\bar{D}_{4+}$ we only need (cf. eq. C.1.12)

$$
4 \mathrm{i} \mathcal{I}_{1}^{2} \mathcal{I}_{2}{ }^{-1} \bar{D}_{4+} I_{222}=4 \mathrm{i} \mathcal{I}_{1}\left(\theta_{34} \tilde{\mathrm{x}}_{34}^{-1}-\theta_{14} \tilde{\mathrm{x}}_{14}^{-1}\right)
$$

because the derivative of $I_{0}$ vanishes. There is one nilpotent invariant, which has the same derivative up to a factor of cross ratios. From eq. C.1.10 we get

$$
\bar{D}_{4+} I_{212} \stackrel{\text { l.o. }}{=} \frac{x_{12}^{2} x_{34}^{2}}{x_{13}{ }^{2} x_{24}{ }^{2}}\left(\theta_{34} \tilde{\mathrm{x}}_{34}^{-1}-\theta_{14} \tilde{\mathrm{x}}_{14}^{-1}\right)
$$

With the appropriate factor these two derivatives cancel and furthermore $D_{2-} I_{212}=$ 0 and $\bar{D}_{3+} I_{212}=0$, so that it does not influence the cancellation of the derivatives of $\mathcal{I}_{1}$. Finally the derivative $D_{1-}$ of the resulting combination is

$$
\begin{array}{ll} 
& -4 \mathrm{i} \mathcal{I}_{1}\left(\mathcal{I}_{2}{ }^{-1} D_{1-} I_{0}+\mathcal{I}_{1} \mathcal{I}_{2}{ }^{-1} D_{1-} I_{222}-\mathcal{I}_{1} \mathcal{I}_{2}{ }^{-1} D_{1-} I_{212}\right) \\
\stackrel{\text { l.o. }}{=} & -4 \mathrm{i} \mathcal{I}_{1}\left(-\tilde{\mathrm{x}}_{12}^{-1} \bar{\theta}_{12}+\tilde{\mathrm{x}}_{13}^{-1} \bar{\theta}_{13}-\tilde{\mathrm{x}}_{13}^{-1} \bar{\theta}_{13}+\tilde{\mathrm{x}}_{14}^{-1} \bar{\theta}_{14}+\tilde{\mathrm{x}}_{12}^{-1} \bar{\theta}_{12}-\tilde{\mathrm{x}}_{14}^{-1} \bar{\theta}_{14}\right) \\
= & 0 .
\end{array}
$$


Thus we have our special solution, but there may still be possible ways, in which the derivatives of the nilpotent invariants with appropriate factors of cross ratios cancel on their own. These would have to appear in the most general solution to our problem. So we define the functions $f_{1, l}\left(\mathcal{I}_{1}, \mathcal{I}_{2}\right)$ as a term canceling the derivative of $f_{0,1}$ and the rest:

$$
\begin{aligned}
& f_{1,1}=-4 \mathrm{i} \mathcal{I}_{1} \mathcal{I}_{2}-1 \frac{\partial f_{0,1}}{\partial \mathcal{I}_{1}}+g_{1,1} \\
& f_{1,2}=4 \mathrm{i} \mathcal{I}_{1} \mathcal{I}_{2}{ }^{-1} \frac{\partial f_{0,1}}{\partial \mathcal{I}_{1}}+g_{1,2} \\
& f_{1,3}=4 \mathrm{i} \mathcal{I}_{1} \mathcal{I}_{2}{ }^{-1} \frac{\partial f_{0,1}}{\partial \mathcal{I}_{1}}+g_{1,3} \\
& f_{1,4}=-4 \mathrm{i} \mathcal{I}_{1} \mathcal{I}_{2}{ }^{-1} \frac{\partial f_{0,1}}{\partial \mathcal{I}_{1}}+g_{1,4} \\
& f_{1,5}=4 \mathrm{i} \mathcal{I}_{1} \mathcal{I}_{2}{ }^{-1} \frac{\partial f_{0,1}}{\partial \mathcal{I}_{1}}+g_{1,5} \\
& f_{1,6}=-4 \mathrm{i} \mathcal{I}_{1}\left(\mathcal{I}_{2}{ }^{-1}-\mathcal{I}_{1} \mathcal{I}_{2}{ }^{-1}\right) \frac{\partial f_{0,1}}{\partial \mathcal{I}_{1}}+g_{1,6} \\
& f_{1,7}=-4 \mathrm{i} \mathcal{I}_{1} \mathcal{I}_{2}{ }^{-1} \frac{\partial f_{0,1}}{\partial \mathcal{I}_{1}}+g_{1,7} \\
& f_{1,8}=4 \mathrm{i} \mathcal{I}_{1}\left(\mathcal{I}_{2}{ }^{-1}-\mathcal{I}_{1} \mathcal{I}_{2}{ }^{-1}\right) \frac{\partial f_{0,1}}{\partial \mathcal{I}_{1}}+g_{1,8}
\end{aligned}
$$

At this point we will interrupt the proof of theorem 5.1 in order to show, that all functions $g_{1, l}$ are zero.

Lemma 5.1. Let $g_{1, l}\left(\mathcal{I}_{1}, \mathcal{I}_{2}\right)$ be functions of non-nilpotent invariants, so that

$$
\sum_{l=1}^{8} g_{1, l} \mathfrak{D I}_{1, l}=0
$$

then

$$
\forall l \in\{1, \ldots, 8\}: g_{1, l}=0 .
$$

The vanishing derivative seems to be a weaker condition than in lemma 4.2. Still we find here, that it is actually equivalent.

Proof. Due to the product rule we have

$$
\sum_{l=1}^{8} g_{1, l} \mathfrak{D} \mathfrak{I}_{1, l}=\mathfrak{D} \sum_{l=1}^{8} g_{1, l} \mathfrak{I}_{1, l}-\sum_{l=1}^{8}\left(\mathfrak{D} g_{1, l}\right) \mathfrak{I}_{1, l} .
$$

Only the first order contains the lowest order, so that to lowest order the following equality holds

$$
\sum_{l=1}^{8} g_{1, l} \mathfrak{D}_{1, l} \stackrel{\text { l.o. }}{=} \mathfrak{D} \sum_{l=1}^{8} g_{1, l} . \mathfrak{I}_{1, l} \text {. }
$$


So the derivative of the sum has to be zero. Thus the sum can only contain terms of the form $\theta_{34} \ldots \bar{\theta}_{12}$ apart from the anti-symmetry of the Graßmannian intervals. The only term in all eight nilpotent invariants, $I_{i j k}$, which looks like this, is contained in $I_{212}$ (cf. eq. (C.1.5)-(C.1.12),

$$
I_{212}: \quad \theta_{34} \mathrm{x}_{34} \tilde{\mathrm{x}}_{23} \mathrm{x}_{12} \bar{\theta}_{12} .
$$

This is also the cause, why this invariant has already played a special role above.

Similar to the explanation in section 4.3.3 significant constraints follow just from the terms with three Pauli matrices like this one, because the terms of this type have to be combined in a way to have the desired form as well. If we look at eqns. 4.3.60-4.3.63 and 4.3.65, the only way to achieve this is:

$$
I_{112}+I_{121}-I_{122}+I_{211}-I_{221}
$$

This combination can be completed to the lowest order of $I_{0}$ with the help of the other three invariant.

So we are left with only four invariants, $I_{111}, I_{212}, I_{222}$ and $I_{0}$. When the coefficient functions are redefined appropriately, we can look at linear combinations of these instead of (5.3.39). To get the vanishing derivatives, these combinations may only contains terms of the form, $\theta_{34} \ldots \bar{\theta}_{12}$.

But three of these four invariants only contain terms, $\theta_{i j} \tilde{\mathrm{x}}_{i j}^{-1} \bar{\theta}_{i j}$, with the same indices for $\theta$ and $\bar{\theta}$. Only $I_{212}$ has two other summands, $\theta_{34} \tilde{\mathrm{x}}_{34}^{-1} \bar{\theta}_{24}, \theta_{13} \tilde{\mathrm{x}}_{12}^{-1} \bar{\theta}_{12}$. These two are inappropriate to get from any $\theta_{i j} \tilde{\mathrm{x}}_{i j}^{-1} \bar{\theta}_{i j}$ to the desired form. The coefficient functions have to be zero.

With the help of lemma 5.1 we know, that the special solution given by eqns. (5.3.28)-5.3.35) with $g_{1, l}=0$ is already the general solution of the lowest order of the differential equations, which we have to solve to show theorem 5.1. So all $f_{1, j}$ are fully determined by the $f_{0,1}$.

Now we rewrite this solution in a convenient way. To this order the differential equations are satisfied by

$$
f_{4 P F}=\left(1+4 \mathrm{i} T^{\prime} \mathcal{I}_{1} \frac{\partial}{\partial \mathcal{I}_{1}}\right) f_{0,1}\left(\mathcal{I}_{1}, \mathcal{I}_{2}\right)+\mathcal{O}\left((\theta \bar{\theta})^{2}\right)
$$

where

$$
T^{\prime}=\frac{\mathcal{I}_{6}}{\sqrt{J_{1(1)}}} I_{212}-\frac{\mathcal{I}_{4}}{\sqrt{J_{1(1)}}} I_{222}-\frac{1}{\mathcal{I}_{3} \sqrt{J_{1(1)}}} I_{0}
$$

is a fixed function of four-point invariants. Here the factors we had before are replaced by factors with cross ratios, $\mathcal{I}_{3}, \mathcal{I}_{4}$ and $\mathcal{I}_{6}$ (eqns. 4.2.9, 4.2.10) and (4.2.12), and $J_{1(1)}$ (eq.4.2.8)). These differ only in higher order, which can be done here with the effect, that the coefficient functions in higher orders change correspondingly. The new factors just eliminate the overall prefactors of the nilpotent invariants in all orders, which simplifies calculations in the following. 


\subsubsection{Iterative conclusion to higher Graßmannian orders}

Eq. (5.3.42 shows exactly, what restrictions follow from the lowest non-vanishing (i.e. first) order of the differential equations (5.3.9). If one looks beyond first order of Graßmann variables, one simplifies matters even more going over to a new set of three nilpotent invariants replacing the three appearing in eq. (5.3.43). Using 4.3.9) they are given by eqns. 4.3.147, 4.3.148) and 4.3.164.

They have especially simple expressions in terms of the variables $\theta_{i}, \bar{\theta}_{j}$ and $\tilde{x}_{\bar{i} j}$ and differ only in second and higher orders from those in eq. (5.3.43). Consequently $T^{\prime}$ is replaced by

$$
T=T_{212}-T_{222}-T_{0},
$$

which still solves the differential equations to first order. This new set of invariants is especially suitable because it allows the iteration from lowest to higher orders. We prepare these steps with a lemma. We show the equation, which is the basis of the iterative steps. Afterwards we see, that there are also at higher orders no possible other combinations of invariants, which could appear in the four-point function.

With the new function $T$ we find the following equation, instead of the corresponding one with $T^{\prime}$, which we have found to be only valid in lowest order.

\section{Lemma 5.2.}

$$
\mathfrak{D} \mathcal{I}_{1}=-4 \mathrm{i} \mathcal{I}_{1} \mathfrak{D} T
$$

Proof. The derivatives of the invariants $T_{111}, T_{212}, T_{222}$ and $T_{0}$ can be found in appendix C.2. They add to the derivatives of $T$ :

Plugged into eq. 5.3.45 this already ends this prove.

Now we have to bring eq. 5.3.20 back into our mind. We can write down the corresponding equation with the results we have so far with the new invariants. This is nothing else than the summands with lowest order $\theta$ resp. $\bar{\theta}$ of the derivative of eq. 5.3.42 with $T^{\prime}$ replaced by $T$ :

$$
\mathfrak{D}\left(1+4 \mathrm{i} T \mathcal{I}_{1} \frac{\partial}{\partial \mathcal{I}_{1}}\right) f_{0,1}\left(\mathcal{I}_{1}, \mathcal{I}_{2}\right)=\frac{\partial f_{0,1}}{\partial \mathcal{I}_{1}} \mathfrak{D} \mathcal{I}_{1}+4 \mathrm{i}(\mathfrak{D} T) \mathcal{I}_{1} \frac{\partial f_{0,1}}{\partial \mathcal{I}_{1}}+\mathcal{O}\left((\theta \bar{\theta})^{2}\right) .
$$

The $\mathcal{O}\left((\theta \bar{\theta})^{2}\right)$ here is a summand from the product rule in the second term, which we will get to later. One directly sees, that the two summands cancel because of lemma 5.2. These two summands do not only cancel in their lowest order, but completely as they are written down here. This is remarkable because we just demanded the cancellation of the lowest orders in the derivation of eq. 5.3.42. Because of this fact there are no complicated remainders left by the cancellation in lowest order, as it would have been with the set of invariants we started with.

The coefficient functions have been redefined twice now corresponding to the steps, in which $I_{0}$ was introduced and in which $T^{\prime}$ was replaced by $T$. So we call the so far undetermined coefficient functions $h_{k, l}$ with $k \geq 2$ and have

$$
f_{4 P F}=\left(1+4 \mathrm{i} T \mathcal{I}_{1} \frac{\partial}{\partial \mathcal{I}_{1}}\right) f_{0,1}\left(\mathcal{I}_{1}, \mathcal{I}_{2}\right)+\sum_{k=2}^{4} \sum_{l=1}^{n_{k}} h_{k, l} \Im_{k, l} .
$$


If we now look at the derivative of the whole four point function in eq. (5.3.13) we are left with half the product rule of eq. (5.3.46) and the other terms of order $(\theta \bar{\theta}) \theta$ resp. $(\theta \bar{\theta}) \bar{\theta}$ and higher. If we plug in eq. (5.3.45), we get the following:

$$
\begin{aligned}
\mathfrak{D} f_{4 P F}= & 16 \mathcal{I}_{1} \frac{\partial}{\partial \mathcal{I}_{1}}\left(\mathcal{I}_{1} \frac{\partial f_{0,1}}{\partial \mathcal{I}_{1}}\right) T \mathfrak{D} T+\sum_{i=2}^{3} \sum_{j=1}^{n_{i}}\left(\mathfrak{D} h_{i, j}\right) \mathfrak{I}_{i, j} \\
& +\sum_{k=2}^{4} \sum_{l=1}^{n_{k}} h_{k, l} \mathfrak{D} \mathfrak{I}_{k, l} .
\end{aligned}
$$

Exterminating the lowest order left here $((\theta \bar{\theta}) \theta$ resp. $(\theta \bar{\theta}) \bar{\theta})$, which has to be zero, we can write down an equation with one side, which depends only on $T, \mathcal{I}_{1}$ and $\mathcal{I}_{2}$ (as argument of $f_{0,1}$ ):

$$
\sum_{l=1}^{18} h_{2, l} \mathfrak{D I}_{2, l} \stackrel{\text { l.o. }}{=}-16 \mathcal{I}_{1} \frac{\partial}{\partial \mathcal{I}_{1}}\left(\mathcal{I}_{1} \frac{\partial f_{0,1}}{\partial \mathcal{I}_{1}}\right) T \mathfrak{D} T .
$$

Thus there is the following special solution for the coefficient functions $h_{2, l}$ on the left hand side, in which they also only depend on these three invariants,

$$
\sum_{l=1}^{18} h_{2, l} \Im_{2, l} \stackrel{\text { l.o. }}{=}-8\left(T \mathcal{I}_{1} \frac{\partial}{\partial \mathcal{I}_{1}}\right)^{2} f_{0,1}\left(\mathcal{I}_{1}, \mathcal{I}_{2}\right)
$$

Now we have to discuss the question of uniqueness of the solution. That is tantamount to the proof of the following lemma.

Lemma 5.3. Let $\alpha_{l}\left(\mathcal{I}_{1}, \mathcal{I}_{2}\right)$ be functions of these two invariants, so that

$$
\sum_{l=1}^{18} \alpha_{l} \mathfrak{D I}_{2, l} \stackrel{\text { l.o. }}{=} 0
$$

then

$$
\forall l \in\{1, \ldots, 18\}: \alpha_{l}=0 .
$$

This is not obvious, although we have proven already lemma 4.4. As we have seen in the proof of lemma 5.1, it is in principle possible, that such a combination of the derivatives of nilpotent invariants vanished, while the combination with the same coefficient functions of only the nilpotent invariants does not.

The proof of this lemma can be found in appendix C.3.1. It consists of a lengthy calculation, which is not needed for the argumentation here.

Here we can again redefine the higher order coefficients $h_{3, l}$ and $h_{4,1}$, so that the general solution of the differential equations, which we solve here, can be written down in the following form:

$$
f_{4 P F}=\left(1+4 \mathrm{i} T \mathcal{I}_{1} \frac{\partial}{\partial \mathcal{I}_{1}}-8\left(T \mathcal{I}_{1} \frac{\partial}{\partial \mathcal{I}_{1}}\right)^{2}\right) f_{0,1}\left(\mathcal{I}_{1}, \mathcal{I}_{2}\right)+\sum_{k=3}^{4} \sum_{l=1}^{n_{k}} h_{k, l}^{\prime} \Im_{k, l}
$$


If we take the derivative of this, we find, that one term of the product rule of the new term is of lowest order 3 in $\theta \bar{\theta}$ and is left after the cancellations following lemma 5.2. This term comes from the derivative of all but $T^{2}$ :

$$
-8 T^{2}\left[\frac{\partial}{\partial \mathcal{I}_{1}}\left(\left(\mathcal{I}_{1} \frac{\partial}{\partial \mathcal{I}_{1}}\right)^{2} f_{0,1}\left(\mathcal{I}_{1}, \mathcal{I}_{2}\right)\right)\right]\left(-4 \mathrm{i} \mathcal{I}_{1} \mathfrak{D} T\right) .
$$

The whole derivative of eq. 5.3.53 is

$$
\begin{aligned}
\mathfrak{D} f_{4 P F}= & 32 \mathrm{i}\left(\mathcal{I}_{1} \frac{\partial}{\partial \mathcal{I}_{1}}\right)^{3} f_{0,1}\left(\mathcal{I}_{1}, \mathcal{I}_{2}\right) T^{2} \mathfrak{D} T+\sum_{j=1}^{n_{i}}\left(\mathfrak{D} h_{i, j}\right) \mathfrak{I}_{i, j} \\
& +\sum_{k=3}^{4} \sum_{l=1}^{n_{k}} h_{k, l} \mathfrak{D I}_{k, l} .
\end{aligned}
$$

In the same way as for the last step we get the term of the next order as the solution from the lowest order of this derivative again,

$$
\sum_{l=1}^{8} h_{3, l} \mathfrak{D I}_{3, l} \stackrel{\text { l.o. }}{=}-32 \mathrm{i}\left(\mathcal{I}_{1} \frac{\partial}{\partial \mathcal{I}_{1}}\right)^{3} f_{0,1}\left(\mathcal{I}_{1}, \mathcal{I}_{2}\right) T^{2} \mathfrak{D} T,
$$

so that we can just write it down:

$$
\begin{aligned}
f_{4 P F}= & \left(1+4 \mathrm{i} T \mathcal{I}_{1} \frac{\partial}{\partial \mathcal{I}_{1}}-8\left(T \mathcal{I}_{1} \frac{\partial}{\partial \mathcal{I}_{1}}\right)^{2}-\frac{32}{3} \mathrm{i}\left(T \mathcal{I}_{1} \frac{\partial}{\partial \mathcal{I}_{1}}\right)^{3}\right) f_{0,1}\left(\mathcal{I}_{1}, \mathcal{I}_{2}\right) \\
& +h_{4,1}^{\prime \prime} \mathfrak{I}_{4,1}
\end{aligned}
$$

We only need to look at the uniqueness of the solution also here:

Lemma 5.4. Let $\alpha_{l}\left(\mathcal{I}_{1}, \mathcal{I}_{2}\right)$ be functions of these two invariants, so that

$$
\sum_{l=1}^{8} \alpha_{l} \mathfrak{D I}_{3, l} \stackrel{\text { l.o. }}{=} 0
$$

then

$$
\forall l \in\{1, \ldots, 8\}: \alpha_{l}=0 .
$$

Also the proof of this lemma is given in the appendix in section C.3.2.

For the last step we take the derivative of eq. 5.3.57):

$$
\mathfrak{D} f_{4 P F}=-\frac{32}{3} 4\left(\mathcal{I}_{1} \frac{\partial}{\partial \mathcal{I}_{1}}\right)^{4} f_{0,1}\left(\mathcal{I}_{1}, \mathcal{I}_{2}\right) T^{3} \mathfrak{D} T+h_{k, l} \mathfrak{D} \mathfrak{I}_{4,1} .
$$

Here there is no difference between the lowest order and the exact result, as no terms in higher orders are possible due to the nilpotency of $T$. Hence the function $f_{4 P F}$ is

$$
\begin{aligned}
f_{4 P F}= & \left(1+4 \mathrm{i} T \mathcal{I}_{1} \frac{\partial}{\partial \mathcal{I}_{1}}-8\left(T \mathcal{I}_{1} \frac{\partial}{\partial \mathcal{I}_{1}}\right)^{2}-\frac{32}{3} \mathrm{i}\left(T \mathcal{I}_{1} \frac{\partial}{\partial \mathcal{I}_{1}}\right)^{3}\right. \\
& \left.+\frac{32}{3}\left(T \mathcal{I}_{1} \frac{\partial}{\partial \mathcal{I}_{1}}\right)^{4}\right) f_{0,1}\left(\mathcal{I}_{1}, \mathcal{I}_{2}\right) .
\end{aligned}
$$


As this is the expansion of the exponential in eq. (5.3.11), this finishes the proof of theorem 5.1 .

The dependence on nilpotent invariants has been totally fixed and the superconformal four-point function of scalar chiral and anti-chiral superfields is left with no more degrees of freedom than the scalar conformal four point function.

The four point function in eq. 5.3.10 is not explicitly given in terms of the chiral and anti-chiral coordinates $z_{1-}, z_{2-}, z_{3+}$ and $z_{4+} . \mathcal{I}_{1}$ and the differential operator $\mathcal{D}$ depend on $\theta_{2}$ and $\bar{\theta}_{3}$. These terms, of course, cancel.

Theorem 5.1 has to be seen here as the link to integrate the scalar chiral/antichiral four point function into the system of nilpotent invariants, which we have analyzed in the last chapter. The proof includes the analysis of derivatives of these nilpotent invariants, which could be transferred to chiral/anti-chiral four point functions, which are not scalar.

\subsubsection{Expression with one other non-nilpotent invariant}

We investigate now, how the four-point function looks, if it is expressed in terms of a different choice of non-nilpotent invariants. More specifically, the new choice consists of a different pair of superconformal cross ratio. As the superconformal cross ratios - just as the conformal ones - have the property, that one goes over into another one under permutation of the indices, we just look at the expressions of the rest of the four point function under such a permutation.

The example we look at here is the exchange of $\mathcal{I}_{1}$ by $\mathcal{I}_{6}$. This is especially interesting for the next chapter because conformal four point functions are given by the inverses of the zeroth order of $\mathcal{I}_{2}$ and $\mathcal{I}_{6}$, for example, in the paper [NRT05] about the partial wave expansion of scalar global conformal four-point functions. The coefficients of the results there can then be directly compared to the superconformal four-point function here.

We start with a look at the four-point function as in eq. (5.3.7). The following equation expresses the replacement, which we want to perform:

$$
f_{4 P F}\left(\mathcal{I}_{1}, \mathcal{I}_{2}, I_{111}, \ldots, I_{222}\right)=f_{4 P F}^{(6)}\left(\mathcal{I}_{6}, \mathcal{I}_{2}, I_{111}, \ldots, I_{222}\right) .
$$

But we do not start with the function on the left side and plug in $\mathcal{I}_{1}$ as a function of $\mathcal{I}_{6}, \mathcal{I}_{2}$, and $I_{i j k}$, which we could get from eq. 4.3.142, if we solve it for $\mathcal{I}_{1}$ with the help of iterative insertion.

We rather take the iteration equation, eq. (5.3.45), and interchange the indices 3 and 4 in it. Thereby $\mathcal{I}_{1}$ gets $\mathcal{I}_{6}$ and we get a new function $T^{(6)}$ instead of $T$,

$$
\mathfrak{D I} \mathcal{I}_{6}=-4 \mathrm{i} \mathcal{I}_{6} \mathfrak{D} T^{(6)} \text {. }
$$

As the third and fourth derivative, which $\mathfrak{D}$ stands for, are $\bar{D}_{3+}$ and $\bar{D}_{4+}$, only the corresponding two equations switch. This also shows the limits, in which the superconformal cross ratios can be easily replaced, because we need exactly these equations with the derivatives $\mathfrak{D}$ for the four point function, eq. (5.3.7), and may not exchange indices between chiral and anti-chiral fields. 
Now we interchange the indices in the function $T=T_{222}+T_{0}-T_{212}$. For the first two summands this is simple, as $T_{222}$ gets $-T_{222}$ using (A.2.12) and $T_{0}$ gets $T_{111}$ (cf. eqns. 4.3.147), (4.3.148) and (4.3.144)). The replacement of $T_{212}$, however, is a nilpotent invariant, which we have not given a name so far and now name $T_{212}^{(6)}$. Its expression with superconformal intervals is 4.3.164) with the indices replaced. But we learn more about it, if we plug eq. (4.3.4) into (4.3.154) and change the variables therein. We see, that the appearing three point variables $\tilde{\mathrm{X}}_{1(2)-}$ (eq. 4.2.4), $\tilde{\Theta}_{1(1)}$ (eq. 4.2.18) and $\tilde{\bar{\Theta}}_{1(2)}$ (eq. 4.2.19) change to the variables $\tilde{\mathrm{X}}_{1(2)+}, \tilde{\Theta}_{1}$ and $-\tilde{\bar{\Theta}}_{1(2)}$, respectively. So, if we define

$$
T_{212}^{(6)}=I_{212}^{(6)}-2 \mathrm{i} I_{212}^{(6)}{ }^{2}
$$

we have

$$
I_{212}^{(6)}=\frac{\tilde{\bar{\Theta}}_{1(2)} \tilde{X}_{1(2)+} \tilde{\Theta}_{1}}{X_{1(2)+}{ }^{2}} .
$$

With eq. 4.2.26 and the relation of $\tilde{\mathrm{X}}_{1(2)+}$ and $\tilde{\mathrm{X}}_{1(2)-}$ analogues to eq. 4.1.13) we can rewrite this in terms of invariants $I_{i j k}$ and $\hat{X}_{1(i) \pm}^{2}$,

$$
\begin{aligned}
I_{212}^{(6)} & =\frac{\tilde{\Theta}_{1(2)}\left(\tilde{X}_{1(2)-}+4 \mathrm{i} \bar{\Theta}_{1(2)} \Theta_{1(2)}\right)\left(\tilde{\Theta}_{1(1)}-\tilde{\Theta}_{1(2)}\right)}{X_{1(2)+}{ }^{2}} \\
& =-\frac{I_{212}}{X_{1(2)+}{ }^{2}}+\frac{I_{222}}{\hat{X}_{1(2)+}^{2}}+8 \mathrm{i} \frac{I_{112} I_{122}}{\hat{X}_{1(1)+}^{2} \hat{X}_{1(2)+}^{2}}-8 \mathrm{i} \frac{I_{122}{ }^{2}}{\hat{X}_{1(1)+}^{2} \hat{X}_{1(2)+}^{2}} .
\end{aligned}
$$

In the last line we used eq. 4.3.25). Plugged into eq. 5.3 .63 it is possible to simplify the result with the help of eq. 4.3.43). Also one could replace $\hat{X}_{1(1)+}^{2}$ by $I_{111}$ with eq. 4.3.113. But at this point this is not significant.

We now need to see, that the iteration works also with the new equation 5.3.62 and gives us a very similar result in the new variables in comparison to the old ones. The starting point is an expansion of the function $f_{4 P F}^{(6)}$ as in theorem 4.1 , but with $\mathcal{I}_{1}$ replaced by $\mathcal{I}_{6}$. This expansion exists and is unique, because it is a simple corollary of this theorem, that any independent pair of non-nilpotent invariants can replace $\mathcal{I}_{1}$ and $\mathcal{I}_{2}$ in this expansion. $\mathcal{I}_{1}$ and $\mathcal{I}_{2}$ can always be given as a function of the new pair of non-nilpotent invariants and the nilpotent monomials, $\mathfrak{I}_{p, q}$, which can be plugged into the expansion in theorem 4.1.

We get the analogous version of eq. 5.3.13,

$$
\mathfrak{D} f_{4 P F}^{(6)}=\sum_{i=0}^{3} \sum_{j=1}^{n_{i}} \frac{\partial f_{i, j}^{(6)}\left(\mathcal{I}_{6}, \mathcal{I}_{2}\right)}{\partial \mathcal{I}_{6}}\left(\mathfrak{D} \mathcal{I}_{6}\right) \mathfrak{I}_{i, j}+\sum_{k=1}^{4} \sum_{l=1}^{n_{k}} f_{k, l}^{(6)}\left(\mathcal{I}_{6}, \mathcal{I}_{2}\right) \mathfrak{D} \mathfrak{I}_{k, l},
$$

where $f_{i, j}^{(6)}\left(\mathcal{I}_{6}, \mathcal{I}_{2}\right)$ are the coefficient functions of the expansion of $f_{4 P F}^{(6)}$. Because the calculation has just two indices exchanged, it is clear, that as in the proof of 
theorem 5.1 all coefficient functions $f_{1, j}^{(6)}$ have to be determined by $f_{0,1}^{(6)}$, so that the derivatives cancel in lowest order. This leads to (cf. eq. (5.3.47))

$$
f_{4 P F}^{(6)}=\left(1+4 \mathrm{i} T^{(6)} \mathcal{I}_{6} \frac{\partial}{\partial \mathcal{I}_{6}}\right) f_{0,1}^{(6)}\left(\mathcal{I}_{6}, \mathcal{I}_{2}\right)+\mathcal{O}\left((\theta \bar{\theta})^{2}\right) .
$$

As just a change of variables is discussed here, which cannot add degrees of freedom, we skip most part of the proof of theorem 5.1 about vanishing linear combination and the iteration then is straight forward. Eq. (5.3.62) leads three times to the next higher order and we get analogously to eq. (5.3.60

$$
\begin{aligned}
f_{4 P F}^{(6)}= & \left(1+4 \mathrm{i} T^{(6)} \mathcal{I}_{6} \frac{\partial}{\partial \mathcal{I}_{6}}-8\left(T^{(6)} \mathcal{I}_{6} \frac{\partial}{\partial \mathcal{I}_{6}}\right)^{2}-\frac{32}{3} \mathrm{i}\left(T^{(6)} \mathcal{I}_{6} \frac{\partial}{\partial \mathcal{I}_{6}}\right)^{3}\right. \\
& \left.+\frac{32}{3}\left(T^{(6)} \mathcal{I}_{6} \frac{\partial}{\partial \mathcal{I}_{6}}\right)^{4}\right) f_{0,1}\left(\mathcal{I}_{6}, \mathcal{I}_{2}\right)
\end{aligned}
$$

Finally we write the bracket as an exponential and have the four-point function in the new form:

$$
\left\langle\bar{\Phi}_{1}\left(z_{1-}\right) \bar{\Phi}_{2}\left(z_{2-}\right) \Phi_{3}\left(z_{3+}\right) \Phi_{4}\left(z_{4+}\right)\right\rangle=\underbrace{e^{4 \mathrm{i} T^{(6)} \mathcal{I}_{6} \frac{\partial}{\partial \mathcal{I}_{6}}}}_{\mathcal{D}^{(6)}} \frac{f_{0,1}^{(6)}\left(\mathcal{I}_{6}, \mathcal{I}_{2}\right)}{x_{\overline{1} 3}{ }^{2\left(\eta_{3}-\eta_{2}\right)} x_{\overline{1} 4}{ }^{2 \eta_{4}} x_{\overline{2} 3}{ }^{2 \eta_{2}}} .
$$

So the change from the function $f_{0,1}\left(\mathcal{I}_{1}, \mathcal{I}_{2}\right)$ to $f_{0,1}^{(6)}\left(\mathcal{I}_{6}, \mathcal{I}_{2}\right)$ is compensated by the one from $T$ to $T^{(6)}$.

\subsubsection{Rational four-point functions}

In the remaining part of this chapter the case of rational four-point function is discussed, which leads to an expression containing only $z_{1-}, z_{2-}, z_{3+}$ and $z_{4+}$. It was shown in [NT01, that in global conformal field theory all correlation functions are rational. As a consequence of global conformal symmetry of the correlation functions of component fields this is also the case for supersymmetric theories with this spacetime symmetry. If the correlation functions of all combinations of component fields of a multiplet are rational, the correlation function of the corresponding superfield also has to be rational.

In rational four-point functions the function $f_{0,1}$ is a power series:

$$
f_{0,1}\left(\mathcal{I}_{1}, \mathcal{I}_{2}\right)=\sum_{k_{1}, k_{2}} f_{0,1, k_{1}, k_{2}} \mathcal{I}_{1}^{k_{1}} \mathcal{I}_{2}{ }^{k_{2}}
$$

The differential operator applied to this leads to

$$
\mathcal{D} f_{0,1}\left(\mathcal{I}_{1}, \mathcal{I}_{2}\right)=\sum_{k_{1}, k_{2}} f_{0,1, k_{1}, k_{2}} \underbrace{e^{4 \mathrm{i} T k_{1}} \mathcal{I}_{1}^{k_{1}}}_{\equiv \mathcal{I}_{1}^{\prime}} \mathcal{I}_{2}^{k_{2}} .
$$

This is rewritten now as a function of chiral and anti-chiral variables. We also define here the invariant $\mathcal{I}_{1}^{\prime}$, which we simplify and get an expression in terms of 
these variables in eq. (5.3.76). Only $\theta_{34}, \theta_{21}$ and $x_{\bar{i} j}$ with $i=1,2$ and $j=3,4$ may appear. Because of the exponential function in (5.3.71) it is useful to express $x_{\overline{1} 2}$, $x_{\overline{3} 4}$ and $x_{\overline{3} 2}$ by the "allowed "intervals times an exponential function with nilpotent exponent. One finds, that

$$
\begin{aligned}
x_{\overline{1} 2}^{2} & =\left(x_{\overline{1} 3}^{\mu}-x_{\overline{2} 3}^{\mu}\right)^{2} e^{4 \mathrm{i}\left(\theta_{23} \tilde{\mathrm{x}}_{\overline{1} 2}^{-1} \bar{\theta}_{21}-2 \mathrm{i}\left(\theta_{23} \tilde{\mathrm{x}}_{\overline{1} 2}^{-1} \bar{\theta}_{21}\right)^{2}\right)}, \\
x_{\overline{3} 4}^{2} & =\left(x_{\overline{1} 3}^{\mu}-x_{\overline{1} 4}^{\mu}\right)^{2} e^{4 \mathrm{i}\left(\theta_{43} \tilde{\tilde{x}}_{\overline{3} 4}^{-1} \bar{\theta}_{13}-2 \mathrm{i}\left(\theta_{43} \tilde{\mathrm{x}}_{\overline{3} 4}^{-1} \bar{\theta}_{13}\right)^{2}\right)}, \\
x_{\overline{3} 2}^{2} & =x_{\overline{2} 3}^{2} e^{-4 \mathrm{i}\left(\rho_{23}-2 \mathrm{i} \rho_{23}{ }^{2}\right)}
\end{aligned}
$$

using $\rho_{23}$ from eq. 4.3 .9 .

This is inserted in the cross ratio $\mathcal{I}_{1}$. The exponents in eqns. 5.3.72)-5.3.74 multiplied with these powers cancel most of the exponent in eq. (5.3.71). The following four-point function remains:

$$
\left\langle\bar{\Phi}_{1}\left(z_{1-}\right) \bar{\Phi}_{2}\left(z_{2-}\right) \Phi_{3}\left(z_{3+}\right) \Phi_{4}\left(z_{4+}\right)\right\rangle=\frac{\sum_{k_{1}, k_{2}} f_{0, k_{1}, k_{2}} \mathcal{I}_{1}^{\prime k_{1}} \mathcal{I}_{2}{ }^{k_{2}}}{x_{\overline{1} 3}{ }^{2\left(\eta_{3}-\eta_{2}\right)} x_{\overline{14}}{ }^{2 \eta_{4}} x_{\overline{2} 3}{ }^{2 \eta_{2}}} .
$$

with

$$
\mathcal{I}_{1}^{\prime}=e^{4 \mathrm{i}\left(\gamma-2 \mathrm{i} \gamma^{2}\right)} \frac{x_{\overline{1} 4}^{2} x_{\overline{2} 3}^{2}}{\left(x_{\overline{1} 3}^{\mu}-x_{\overline{2} 3}^{\mu}\right)^{2}\left(x_{\overline{1} 3}^{\mu}-x_{\overline{1} 4}^{\mu}\right)^{2}}
$$

and

$$
\gamma=\frac{\theta_{34} \mathrm{x}_{\overline{1} 4} \tilde{\mathrm{x}}_{\overline{1} 3} \mathrm{x}_{\overline{2} 3} \bar{\theta}_{21}}{\left(x_{\overline{1} 3}^{\mu}-x_{\overline{2} 3}^{\mu}\right)^{2}\left(x_{\overline{1} 3}^{\mu}-x_{\overline{1}}^{\mu}\right)^{2}}-\frac{x_{\overline{1} 3}^{2}\left(\theta_{34} \mathrm{x}_{\overline{2} 3} \bar{\theta}_{21}+\theta_{34} \mathrm{x}_{\overline{1} 4} \bar{\theta}_{21}-\theta_{34} \mathrm{x}_{\overline{1} 3} \bar{\theta}_{21}\right)}{\left(x_{\overline{1} 3}^{\mu}-x_{\overline{2} 3}^{\mu}\right)^{2}\left(x_{\overline{1} 3}^{\mu}-x_{\overline{1} 4}^{\mu}\right)^{2}} .
$$

Setting the Grassmann variables to zero, the exponent in (5.3.76) vanishes and thus $\mathcal{I}_{1}^{\prime}$ passes into a conformal cross ratio just like $\mathcal{I}_{2}$ does. Hence the coefficients $f_{0, k_{1}, k_{2}}$ are also those of the four-point function of the lowest component fields, which are scalar conformal fields.

If we now look again at the four-point function expressed with the superconformal cross ratio $\mathcal{I}_{6}$ instead of $\mathcal{I}_{1}$, we see, that the differential operator $\mathcal{D}^{(6)}$ and the function $f_{0,1}^{(6)}$ in eq. 5.3 .69 result from the interchange of the indices 3 and 4 in comparison to the case above. Thus applying this differential operator to the series expension of this function is the same calculation with different indices and results in

$$
\left\langle\bar{\Phi}_{1}\left(z_{1-}\right) \bar{\Phi}_{2}\left(z_{2-}\right) \Phi_{3}\left(z_{3+}\right) \Phi_{4}\left(z_{4+}\right)\right\rangle=\frac{\sum_{k_{1}, k_{2}} f_{0, k_{1}, k_{2}}^{(6)}\left(\mathcal{I}_{6}^{\prime-1}\right)^{k_{1}} \mathcal{I}_{2}^{k_{2}}}{x_{\overline{1} 3}{ }^{2\left(\eta_{3}-\eta_{2}\right)} x_{\overline{1} 4^{2 \eta_{4}} x_{\overline{2} 3}{ }^{2 \eta_{2}}}} .
$$

with

$$
\mathcal{I}_{6}^{\prime-1}=e^{-4 \mathrm{i}\left(\gamma^{(6)}-2 \mathrm{i}\left(\gamma^{(6)}\right)^{2}\right)} \frac{\left(x_{\overline{1} 4}^{\mu}-x_{\overline{2} 4}^{\mu}\right)^{2}\left(x_{\overline{1} 3}^{\mu}-x_{\overline{1} 4}^{\mu}\right)^{2}}{x_{\overline{1} 3}^{2} x_{\overline{2} 4}^{2}} \quad, \quad \mathcal{I}_{2}=\frac{x_{\overline{1} 4}^{2} x_{\overline{2} 3}^{2}}{x_{\overline{1} 3}^{2} x_{\overline{2} 4}^{2}}
$$


and

$$
\gamma^{(6)}=\frac{\theta_{43} \mathrm{x}_{\overline{1} 3} \tilde{\mathrm{x}}_{\overline{1} 4} \mathrm{x}_{\overline{2} 4} \bar{\theta}_{21}}{\left(x_{\overline{1} 4}^{\mu}-x_{\overline{2} 4}^{\mu}\right)^{2}\left(x_{\overline{1} 3}^{\mu}-x_{\overline{1} 4}^{\mu}\right)^{2}}-\frac{x_{\overline{1} 4}^{2}\left(\theta_{43} \mathrm{x}_{\overline{2} 4} \bar{\theta}_{21}+\theta_{43} \mathrm{x}_{\overline{1} 3} \bar{\theta}_{21}-\theta_{43} \mathrm{x}_{\overline{1} 4} \bar{\theta}_{21}\right)}{\left(x_{\overline{1} 4}^{\mu}-x_{\overline{2} 4}^{\mu}\right)^{2}\left(x_{\overline{1} 3}^{\mu}-x_{\overline{1} 4}^{\mu}\right)^{2}} .
$$

Here we have switched over from $\mathcal{I}_{6}^{\prime}$ to $\mathcal{I}_{6}^{\prime-1}$ by changing the sign of the index $k_{1}$. Now the two variables of the expansion, $\mathcal{I}_{6}^{\prime}$ and $\mathcal{I}_{2}$, do not only pass into any conformal cross ratios, but into those in eq. 2.2.6) used in the expressions of global conformal invariant four-point functions in that chapter, when the Graßmann variables are set to zero.

Finally we want to give the relation between the coefficients $f_{0, k_{1}, k_{2}}^{(6)}$ and $f_{0, k_{1}, k_{2}}$. In order to do so we just need to look at zeroth order in Graßmann variables. The conformal cross ratios $\left(\mathcal{I}_{6}^{\prime-1}\right)_{0}=\eta_{1},\left(\mathcal{I}_{1}^{\prime}\right)_{0}$ and $\left(\mathcal{I}_{2}\right)_{0}=\eta_{2}$ - the index zero denotes the zeroth order part - fulfill the following relation:

$$
\left(\mathcal{I}_{1}^{\prime}\right)_{0}=\left(\mathcal{I}_{2}\right)_{0}\left(\mathcal{I}_{6}^{\prime}\right)_{0}
$$

If we plug this into eq. 5.3 .75 , we can introduce new sumation indices $k_{1}^{\prime}=-k_{1}$ and $k_{2}^{\prime}=k_{1}+k_{2}$, so that

$$
\sum_{k_{1}, k_{2}} f_{0, k_{1}, k_{2}} \mathcal{I}_{1}^{\prime k_{1}} \mathcal{I}_{2} \stackrel{k_{2}}{\stackrel{\text { l.o. }}{=}} \sum_{k_{1}^{\prime}, k_{2}^{\prime}} f_{0,-k_{1}^{\prime}, k_{2}^{\prime}+k_{1}^{\prime}}\left(\mathcal{I}_{6}^{\prime-1}\right)^{k_{1}^{\prime}} \mathcal{I}_{2}{ }^{k_{2}^{\prime}}
$$

As eqns. 5.3.75) and 5.3.78 contain the same correlation function. the expansion on the right hand side must be the one in $(5.3 .78)$ and we can identify

$$
f_{0, k_{1}, k_{2}}^{(6)}=f_{0,-k_{1}, k_{2}+k_{1}} .
$$

The scalar four-point function can be reached without all the nilpotent invariants in this chapter, but then also without the perspective beyond the scalar case. In [PSD11] the four-point function of two chiral and two anti-chiral superfields all having the same scaling dimension are given using a trace invariant and $\mathcal{I}_{2}$. This trace invariant is given by

$$
\operatorname{tr}\left(\mathrm{x}_{\overline{2} 3}{ }^{-1} \mathrm{x}_{\overline{1} 3} \mathrm{x}_{\overline{1} 4}{ }^{-1} \mathrm{x}_{\overline{2} 4}\right)=1-\mathcal{I}_{1}^{\prime-1}+\mathcal{I}_{2}{ }^{-1}
$$

with $\mathcal{I}_{2}$ and $\mathcal{I}_{1}^{\prime}$ from eqns. (4.2.7) and (5.3.76), respectively.

There is another reason, which opposes the use of this trace invariant. If a rational four-point function is written as a power series with this invariant and $\mathcal{I}_{2}$, the coefficients would be resorted in comparison to eq. 5.3.75 and the step from global conformal to global superconformal four-point function would be more complicated. 


\section{Chapter 6}

\section{From conformal to superconformal pole bounds and partial wave expansion}

In this chapter we get back to the result for global conformal field theories in chapter 2. At this point we are now able to transfer these results to global superconformal theories, which means in analogy to global conformal invariance, that these theories are invariant under the single valued action of the superconformal group $S U(2,2 \mid 1)$, whenever both $z$ and the $g z, g \in S U(2,2 \mid 1)$, belong to the superMinkowski space. Representations on covering spaces of Minkowski space (multiplets formulation) respective superspace (superfields formulation) are excluded.

This amounts to global conformal invariances for all component fields in a superconformal superfield. Thus the consequences of global conformal invariance for GCI fields and their correlation functions apply to each such component field. But these fields are not independent. They are connected by supersymmetry transformations: By definition the supersymmetry generators, $Q$ and $\bar{Q}$, relate them. but in section 3.4 .2 we have seen, that also the special conformal generators, $S, \bar{S}$ and $K_{\mu}$, mix the component fields. This mixing goes back to their relation to the supersymmetry generators. So it is sufficient to concentrate on the latter here.

This chapter is devided in two parts. The first is devoted to pole bounds and the second takes a look at the partial wave expansion, which we have discussed in section 2.3. The strategy is similar for both parts. We review, what we know about the component fields from GCI and then see, which consequences the properties of one component field has for the others because of their relation by supersymmetry. This relation is already given by the form of the superfield, which simplifies matters.

\subsection{Pole bounds}

The pole bounds of the correlation functions given in section 2.2 only depend on the represenations the contained fields belong to. Even more the pole bound for a distance of two specific points $x_{i}$ and $x_{j}$ in Minkowski space only depends on the 
representation of the two fields, which are functions of these points in the correlation function. We can write down the bounds following eq. (2.2.3) for all pairs of component fields, which appear in the superconformal correlation function of superfields, if we expand these superfields.

We study the four-point function of a chiral scalar superfield, $\Phi$, and its antichiral hermitian conjugate, $\bar{\Phi}=\Phi^{\dagger}$. Eq. (5.3.78) for four scalar fields of the same scaling dimension $d\left(d=\eta_{2}=\eta_{3}=\eta_{4}\right.$ in 5.3.78) and $\left.d=\eta_{1}=\eta_{3}+\eta_{4}-\eta_{2}\right)$ is

$$
\left\langle\bar{\Phi}\left(x_{1-}, \bar{\theta}_{1}\right) \bar{\Phi}\left(x_{2-}, \bar{\theta}_{2}\right) \Phi\left(x_{3+}, \theta_{3}\right) \Phi\left(x_{4+}, \theta_{4}\right)\right\rangle=\frac{\sum_{k_{1}, k_{2}} f_{0, k_{1}, k_{2}}^{(6)}\left(\mathcal{I}_{6}^{\prime-1}\right)^{k_{1}} \mathcal{I}_{2}{ }^{k_{2}}}{x_{\overline{1} 4}{ }^{2 d} x_{\overline{2} 3}{ }^{2 d}} .
$$

The function $f_{0, k_{1}, k_{2}}^{(6)}$ has been introduced in eq. (5.3.61).

The superfields have the expansion in eqns. (3.4.77) and (3.4.78) in the corresponding chiral and anti-chiral Graßmann variable. Because the four-point function only contains terms with some power of $\theta_{i} \overline{\theta_{j}}$ with $i=3,4$ and $j=1,2$, only nineteen of the in general 81 terms do not vanish. As factors, with which the coefficient functions are multiplied, are not important for our discussion here, we just list the terms in the expansion of eq. 6.1.1 without them:

- $\left\langle\phi^{*}\left(x_{1-}\right) \phi^{*}\left(x_{2-}\right) \phi\left(x_{3+}\right) \phi\left(x_{4+}\right)\right\rangle$,

- $\theta_{3}\left\langle\tilde{\bar{\psi}} \phi^{*} \tilde{\psi} \phi\right\rangle \bar{\theta}_{1}, \theta_{4}\left\langle\tilde{\bar{\psi}} \phi^{*} \phi \tilde{\psi}\right\rangle \bar{\theta}_{1}, \theta_{3}\left\langle\phi^{*} \tilde{\bar{\psi}} \tilde{\psi} \phi\right\rangle \bar{\theta}_{2}, \theta_{4}\left\langle\phi^{*} \tilde{\bar{\psi}} \phi \tilde{\psi}\right\rangle \bar{\theta}_{2}$,

- $\theta_{3} \theta_{4}\langle\tilde{\bar{\psi}} \tilde{\bar{\psi}} \tilde{\psi} \tilde{\psi}\rangle \bar{\theta}_{1} \bar{\theta}_{2}$

- $\theta_{3} \tilde{\theta}_{3}\left\langle m^{*} \phi^{*} m \phi\right\rangle \tilde{\bar{\theta}}_{1} \bar{\theta}_{1}, \theta_{4} \tilde{\theta}_{4}\left\langle m^{*} \phi^{*} \phi m\right\rangle \tilde{\bar{\theta}}_{1} \bar{\theta}_{1}, \theta_{3} \tilde{\theta}_{3}\left\langle\phi^{*} m^{*} m \phi\right\rangle \tilde{\bar{\theta}}_{2} \bar{\theta}_{2}$, $\theta_{4} \tilde{\theta}_{4}\left\langle\phi^{*} m^{*} \phi m\right\rangle \tilde{\bar{\theta}}_{2} \bar{\theta}_{2}$,

- $\theta_{3} \theta_{4}\left\langle m^{*} \phi^{*} \tilde{\psi} \tilde{\psi}\right\rangle \tilde{\bar{\theta}}_{1} \bar{\theta}_{1}, \theta_{3} \theta_{4}\left\langle\phi^{*} m^{*} \tilde{\psi} \tilde{\psi}\right\rangle \tilde{\bar{\theta}}_{2} \bar{\theta}_{2}$,

- $\theta_{3} \tilde{\theta}_{3}\langle\tilde{\bar{\psi}} \tilde{\bar{\psi}} m \phi\rangle \bar{\theta}_{1} \bar{\theta}_{2}, \theta_{4} \tilde{\theta}_{4}\langle\tilde{\bar{\psi}} \tilde{\bar{\psi}} \phi m\rangle \bar{\theta}_{1} \bar{\theta}_{2}$,

- $\left(\theta_{3} \tilde{\theta}_{3}\right) \theta_{4}\left\langle m^{*} \tilde{\bar{\psi}} m \tilde{\psi}\right\rangle\left(\tilde{\bar{\theta}}_{1} \bar{\theta}_{1}\right) \bar{\theta}_{2},\left(\theta_{4} \tilde{\theta}_{4}\right) \theta_{3}\left\langle m^{*} \tilde{\bar{\psi}} \tilde{\psi} m\right\rangle\left(\tilde{\bar{\theta}}_{1} \bar{\theta}_{1}\right) \bar{\theta}_{2}$, $\left(\theta_{3} \tilde{\theta}_{3}\right) \theta_{4}\left\langle\tilde{\bar{\psi}} m^{*} m \tilde{\psi}\right\rangle\left(\tilde{\bar{\theta}}_{2} \bar{\theta}_{2}\right) \bar{\theta}_{1},\left(\theta_{4} \tilde{\theta}_{4}\right) \theta_{3}\left\langle\tilde{\bar{\psi}} m^{*} \tilde{\psi} m\right\rangle\left(\tilde{\bar{\theta}}_{2} \bar{\theta}_{2}\right) \bar{\theta}_{1}$,

- $\left(\theta_{3} \tilde{\theta}_{3}\right)\left(\theta_{4} \tilde{\theta}_{4}\right)\left\langle m^{*} m^{*} m m\right\rangle\left(\tilde{\bar{\theta}}_{1} \bar{\theta}_{1}\right)\left(\tilde{\bar{\theta}}_{2} \bar{\theta}_{2}\right)$.

All these correlation functions of component fields depend on the same chiral variables as the first one. They are not all independent. Each item groups correlation functions, which are related to each other because of the locality of the four-point function of superfields. Here we only take a look at very few of them. We start with the zeroth order, which is the four-point function of complex scalar fields. So we set the Graßmann variables to zero in eq. 6.1.1) and get in lowest, i.e. zeroth order,

$$
\left\langle\phi^{*}\left(x_{1-}\right) \phi^{*}\left(x_{2-}\right) \phi\left(x_{3+}\right) \phi\left(x_{4+}\right)\right\rangle \stackrel{\text { l.o. }}{=} \frac{\sum_{k_{1}, k_{2}} f_{0, k_{1}, k_{2}}^{(6)} s^{k_{1}} t^{k_{2}}}{x_{14}{ }^{2 d} x_{23}{ }^{2 d}} .
$$


We only need the lowest order, because we are only interested in the coefficients, $f_{0, k_{1}, k_{2}}^{(6)}$, which are the same whether we have chiral variables or coordinates of Minkowski space on the right hand side. One could expand the chiral coordinates, which the exact expression of the left hand side contains and has additional to the four-point function of the scalar component fields on Minkowski space also four-point functions of vector fields, which are completely determined by derivatives of the one of the scalar fields (cf. eqns. (3.4.81), (3.4.83) and (3.4.74)). This is the same for the four-point functions of the higher independent component fields, which we listed above, because there is no mixing in the expansions: While in the expansion in chiral variables the indices of $\theta$ and $\bar{\theta}$ are always different, expanding the chiral variables always leads to pairs of $\theta$ and $\bar{\theta}$ with the same indices.

As the pole bounds are more restrictive for the truncated part of the four-point function, we want to know, which summands contain the rest of the four-point function. As we know, that the two-point functions of two scalar fields, $\phi_{i}$ and $\phi_{j}$, are proportional to $x_{i j}{ }^{-2 d}$, one quickly finds

$$
\begin{array}{ll}
\left\langle\phi^{*}\left(x_{1-}\right) \phi^{*}\left(x_{2-}\right)\right\rangle\left\langle\phi\left(x_{3+}\right) \phi\left(x_{4+}\right)\right\rangle: & \left(k_{1}, k_{2}\right)=(-d, d) \\
\left\langle\phi^{*}\left(x_{1-}\right) \phi\left(x_{3+}\right)\right\rangle\left\langle\phi^{*}\left(x_{2-}\right) \phi\left(x_{4+}\right)\right\rangle: & \left(k_{1}, k_{2}\right)=(0, d) \\
\left\langle\phi^{*}\left(x_{1-}\right) \phi\left(x_{4+}\right)\right\rangle\left\langle\phi^{*}\left(x_{2-}\right) \phi\left(x_{3+}\right)\right\rangle: & \left(k_{1}, k_{2}\right)=(0,0)
\end{array}
$$

These poles are generally the maximal poles in the four-point function. The second and the third line considers poles of fields, which actually are in the four-point function. These terms are not in the truncated four-point function, as we also see below, and so the corresponding pole bounds in the truncated four-point function are lower in comparison to the whole four-point function. This is not the case for the first line, but here the two-point functions are already zero. Consequently the term $f_{0,-d, d}^{(6)}$ also does neither appear in the truncated four-point function nor in the whole four-point function.

Now we can write down the poles, which the summands in the truncated fourpoint function have together with the denominator. We also give the bounds, which follow from eq. (2.2.4) in the case of the two fields being $\phi^{*}$ and $\phi$ and from eq. 2.2 .3 and with the fact, that the two-pint function is zero in this case, otherwise:

$$
\begin{aligned}
& \mu_{12}=\mu_{34}=-k_{1} \leq d-1, \\
& \mu_{13}=\mu_{24}=k_{1}+k_{2} \leq d-1, \\
& \mu_{14}=\mu_{23}=d-k_{2} \leq d-1 .
\end{aligned}
$$

Hence the coefficients $f_{0, k_{1}, k_{2}}^{(6)}$ can only be different from zero within the bounds

$$
k_{1} \geq-d+1 \quad, \quad k_{2} \geq 1 \quad \text { and } \quad k_{1}+k_{2} \leq d-1
$$

For $d=4$, which we take as an example here throughout this section, these are 21 possible values in a triangle in the $k_{1}-k_{2}$ plane with corners $\left(k_{1}, k_{2}\right)=(-3,1)$, $(-3,6)$ and $(2,1)$. 
But the coefficients are related by the locality property to each other. If we permute the indices 1 and 2 the four-point function gets

$$
\left\langle\phi^{*}\left(x_{2-}\right) \phi^{*}\left(x_{1-}\right) \phi\left(x_{3+}\right) \phi\left(x_{4+}\right)\right\rangle \stackrel{\text { 1.o. }}{=} \frac{\sum_{k_{1}, k_{2}} f_{0, k_{1}, k_{2}}^{(6)} s^{k_{1}} t^{d-k_{1}-k_{2}}}{x_{14}{ }^{2 d} x_{23}{ }^{2 d}} .
$$

This has to be the same as the original one and by redefining the index $k_{2} \rightarrow$ $d-k_{1}-k_{2}$ we get the relation

$$
f_{0, k_{1}, k_{2}}^{(6)}=f_{0, k_{1}, d-k_{1}-k_{2}}^{(6)} .
$$

This leaves in the case of $d=4$ twelve coefficients independent. The permutation of the indices 3 and 4 leads to the same restriction.

Next we have to determine $\left\langle\tilde{\bar{\psi}} \phi^{*} \tilde{\psi} \phi\right\rangle$. For this we have to expand some terms of eq. 6.1.1). These terms have to have solely the Graßmann variables $\theta_{3}$ and $\bar{\theta}_{1}$, which can either come from $\gamma^{(6)}$, which is part of $\mathcal{I}_{6}^{\prime}$, (eqns. (5.3.79) and (5.3.80) ) or from the expansion of $x_{\overline{1} 3}$. We only need the first order in $\theta_{3} \theta_{1}$ and the zeroth order of all other Graßmann variables. On the way to the expansion of $\mathcal{I}_{6}^{\prime-1}$ we find

$$
\begin{aligned}
\left(x_{\overline{1} 3}^{2}\right)^{n} & =\left(x_{13}^{2}\right)^{n}\left[1-4 \operatorname{in} \frac{\theta_{3} x_{13} \bar{\theta}_{1}}{x_{13}{ }^{2}}\right]+\ldots, \\
\left(x_{\overline{1} 3}^{\mu}-x_{\overline{1} 4}^{\mu}\right)^{2} & =\left(x_{34}^{2}\right)^{n}\left[1-4 \operatorname{in} \frac{\theta_{3} x_{43} \bar{\theta}_{1}}{x_{13}{ }^{2}}\right]+\ldots,
\end{aligned}
$$

The inverse of these terms is given by eq. A.2.10. Thus we get for the more difficult part of $\mathcal{I}_{6}^{\prime-1 k_{1}}$ :

$$
\left[\frac{\left(x_{\overline{1} 4}^{\mu}-x_{\overline{2} 4}^{\mu}\right)^{2}\left(x_{\overline{1} 3}^{\mu}-x_{\overline{1} 4}^{\mu}\right)^{2}}{x_{\overline{1} 3}^{2} x_{\overline{2} 4}^{2}}\right]^{k_{1}}=s^{k_{1}}+4 \mathrm{i} k_{1} \theta_{3}\left(\frac{x_{13}}{x_{13}{ }^{2}}-\frac{x_{43}}{x_{34}{ }^{2}}\right) \bar{\theta}_{1} s^{k_{1}}+\ldots
$$

From the exponential of $\gamma^{(6)}$ we can easily read off the terms:

$$
e^{-4 \mathrm{i} \gamma^{(6)}}=1-4 \mathrm{i} \theta_{3}\left(\frac{x_{13} \tilde{x}_{14} x_{24}-x_{14} 2 \overbrace{\left(x_{24}+x_{13}-x_{14}\right)}^{=x_{23}}}{x_{12}{ }^{2} x_{34}{ }^{2}}\right) \bar{\theta}_{1}+\ldots .
$$

The lowest order of the square of $\gamma^{(6)}$ is already to high to be relevant here. In summary and together with the expansion of $x_{\overline{1} 3}$ in $\mathcal{I}_{2}$ we find, that

$$
\begin{aligned}
2 \theta_{3}\left\langle\tilde{\bar{\psi}} \phi^{*} \tilde{\psi} \phi\right\rangle \bar{\theta}_{1}= & \sum_{k_{1}, k_{2}} 4 \mathrm{i} \theta_{3}\left[\frac{x_{13}}{x_{13}{ }^{2}} k_{2}+\left(\frac{x_{13}}{x_{13}{ }^{2}}-\frac{x_{43}}{x_{34}{ }^{2}}-\frac{x_{13} \tilde{x}_{14} x_{24}-x_{14}{ }^{2} x_{23}}{x_{12}{ }^{2} x_{34}{ }^{2}}\right) k_{1}\right] \bar{\theta}_{1} \\
& \frac{f_{0, k_{1}, k_{2}}^{(6)} s^{k_{1}} t^{d-k_{1}-k_{2}}}{x_{14}{ }^{2 d} x_{23}{ }^{2 d}} .
\end{aligned}
$$

We see, that the expansion has led to factors, which increase the degrees of some of the poles. On the other hand the fermions appearing here are representations of the 
conformal group with $\left(d+\frac{1}{2}, 0, \frac{1}{2}\right)$ and $\left(d+\frac{1}{2}, \frac{1}{2}, 0\right)$. This weakens the pole bounds slightly. This effect could be compensated by the stronger poles from the expansion above, but we find

$$
\begin{aligned}
\mu_{12}=\mu_{34} & =1-k_{1} \leq d, \\
\mu_{13} & =1+k_{1}+k_{2} \leq d, \\
\mu_{14}=\mu_{23} & =d-k_{2} \leq d, \\
\mu_{24} & =k_{1}+k_{2} \leq d-1,
\end{aligned}
$$

and in the end the allowed range is even larger as compared with (6.1.9):

$$
k_{1} \geq-d+1 \quad, \quad k_{2} \geq 0 \quad \text { and } \quad k_{1}+k_{2} \leq d-1 .
$$

A special case is $k_{1}=k_{2}=0$. Here the summand in the eq. 6.1 .16 is zero independent of the coefficient $f_{0, k_{1}, k_{2}}^{(6)}$. But this coefficient has been already excluded in the truncated part of the scalar four-point function in eq. 6.1.2).

The other four-point functions may lead to further restriction. It is not necessary to do the whole expansion and calculate these functions completely. If we look at the appearing terms in 6.1.1), we can already see, which powers of the intervals appear in a specific correlation of function of the component fields.

For example, for $\langle\tilde{\bar{\psi}} \tilde{\bar{\psi}} \tilde{\psi} \tilde{\psi}\rangle$ we have to find the terms, which contain $\theta_{3} \theta_{4} \bar{\theta}_{1} \bar{\theta}_{2}$. Actually we only need the denominators of the canceled down terms. From the square of $\gamma^{(6)}$ we get a factor $1 /\left(x_{12}{ }^{4} x_{34}{ }^{4}\right)$ additional to the poles in the scalar fourpoint function. The expansion of the different appearances of $x_{\bar{i} j}^{2}$ or $\left(x_{\bar{k} l}^{\mu}-x_{\bar{m} n}^{\mu}\right)^{2}$ with $i=k$ or $j=l$ leads to additional factors $1 / x_{i j}{ }^{2}, 1 / x_{k m}{ }^{2}$ or $1 / x_{l n}{ }^{2}$. Thus then poles of the different summands give the following inequalities with the pole bounds for the truncated four-point function:

$$
\begin{aligned}
& \mu_{12}=\mu_{34}=2-k_{1} \leq d, \\
& \mu_{13}=\mu_{24}=1+k_{1}+k_{2} \leq d, \\
& \mu_{14}=\mu_{23}=1+d-k_{2} \leq d .
\end{aligned}
$$

This time we get stricter bounds for $k_{1}$ and $k_{2}$ than in eq. 6.1.9):

$$
k_{1} \geq-d+2 \quad, \quad k_{2} \geq 1 \quad \text { and } \quad k_{1}+k_{2} \leq d-1 .
$$

Finally we want to mention here only one other restriction from a single term in the correlation function $\left\langle m^{*} \phi^{*} m \phi\right\rangle$. This term has to contain $\theta_{3} \tilde{\theta}_{3} \tilde{\bar{\theta}}_{1} \bar{\theta}_{1}$ in eq. 6.1.1). It comes from $\left(\gamma^{(6)}\right)^{2}$. It is easy to see, that there is a part in the numerator, which does not cancel with denominator $x_{12}{ }^{4} x_{34}{ }^{4}$. No term can give a stronger contribution to the following two poles:

$$
\mu_{12}=\mu_{34}=2-k_{1} \leq d
$$

This is equivalent to

$$
k_{1} \geq-d+2
$$


So $k_{1}$ is not more restricted than in (6.1.25). In our example, $d=4,15$ possible summands are left and 9 may be different from each other.

These are the results we obtained so far. In this way one can continue with the rest of the correlation functions of component fields and it seems, that with higher component fields and their correlation functions $k_{1}$ seems to get more and more restricted, as far as one can guess from two steps of stronger restrictions, which we have found so far.

\subsection{Partial wave expansion}

The form of the GCI four-point function of real scalar fields with scaling dimension $d=4$ is known from NRT05. The coefficients of the partial wave expansion were calculated from the five constant in it, But this case is not applicable in the context of chiral and anti-chiral superfields. We have seen in section 3.4.2, that scalar chiral superfields always have a complex scalar field as their lowest component field. In the four-point function of two anti-chiral and two chiral superfields we thus have even for equal scaling dimensions only two pairs of identical fields and these pairs are related by $\bar{\Phi}=\Phi^{\dagger}$.

This leads to more possibly non-vanishing independent amplitudes in the fourpoint function of these four complex scalar fields compared to the case of four real scalar fields discussed in [NRT05]. The reason is, that there are less identities between coefficients because only one identity (eq. (6.1.11)) instead of those in eq. (2.2.7) comes from the locality property.

If one wants to transfer the results of [NRT05] and the constraints from positivity discussed therein, one has to look at other supermultiplets. The general multiplet can be restricted by the condition $V^{\dagger}=V$ to get the general real multiplet.

Still the more general result on partial wave expansions also apply to the fourpoint function of scalar chiral superfields. Also the method to calculate the coefficients of this expansion described in section 2.3 can be used for the explicit calculation of partial wave expansion of the four-point function of four complex scalar fields, as they are contained in the four-point function of superfields.

We have already seen, that the function of cross ratios appearing in the conformal scalar four-point function of the lowest component fields, $\phi$ and $\phi^{*}$, is the same as the one in the superconformal scalar chiral/anti-chiral four-point function of the superfields, $\Phi$ and $\bar{\Phi}$. In the former case this function is evaluated at the conformal cross ratios and in the latter case its arguments are superconformal cross ratios. The cause is the reduction of the superconformal four-point function to the conformal four-point function for vanishing Graßmann variables:

$$
\left.\left\langle\bar{\Phi}_{1} \bar{\Phi}_{2} \Phi_{3} \Phi_{4}\right\rangle\right|_{\theta_{i}=0, \bar{\theta}_{j}=0}=\left\langle\phi_{1}^{*} \phi_{2}^{*} \phi_{3} \phi_{4}\right\rangle=\frac{f(s, t)}{x_{13}{ }^{2\left(\eta_{3}-\eta_{2}\right)} x_{14}{ }^{2 \eta_{4}} x_{23}{ }^{2 \eta_{2}}} .
$$

Because of the global conformal invariance, which we assume here, this function is rational:

$$
f(s, t)=\sum_{k_{1}, k_{2}} f_{0, k_{1}, k_{2}}^{(6)} s^{k_{1}} t^{k_{2}} .
$$


From pole bounds only a finite number of terms may not vanish in this sum. As detailed in section 2.3 the partial wave expansion can be calculated for $\left\langle\phi^{*} \phi^{*} \phi \phi\right\rangle$, if we substitute the cross ratios here by chiral variables, $u$ and $v$, and use expansion formulas to get to the partial wave coefficients. This is, of course, increasingly difficult for more summands in $(6.2 .2)$ and also for higher scaling dimension and less identical fields. A promising eventually non-trivial candidate could be the example from the last section with $d=4$ and fields $\Phi_{3}^{\dagger}=\Phi_{4}^{\dagger}=\bar{\Phi}_{1}=\bar{\Phi}_{2}$.

In the end we have a set of partial wave coefficients, $B_{k L}$, which give the expansion of the superconformal four-point function for vanishing Graßmann variables:

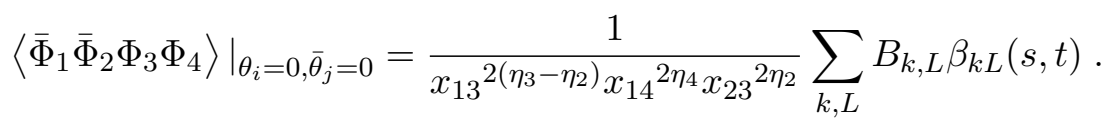

This sum is equal to the function in the numerator in eq. 6.2.1). We have seen in theorem 5.1 , that the whole superconformal four-point function results from the conformal four-point function of the lowest component fields, if we replace the conformal cross ratios by superconformal ones and apply a differential operator, $\mathcal{D}$ to it, which is to the lowest order equal to 1 . But in that theorem the conformal cross ratios have not been $s$ and $t$, yet. Later in section 5.3 .3 we found out, that we have to take the similar differential operator, $\mathcal{D}^{(6)}$, in order to start from the conformal four-point function in terms $s$ and $t$.

This is also true after the expansion into partial waves:

$$
\left\langle\bar{\Phi}_{1} \bar{\Phi}_{2} \Phi_{3} \Phi_{4}\right\rangle=\frac{1}{x_{\overline{1} 3}^{2\left(\eta_{3}-\eta_{2}\right)} x_{\overline{1} 4}^{2 \eta_{4}} x_{\overline{2} 3}{ }^{2 \eta_{2}}} \sum_{k, L} B_{k, L} \mathcal{D}^{(6)} \beta_{k L}\left(\mathcal{I}_{6}{ }^{-1}, \mathcal{I}_{2}\right) .
$$

Because of the rationality of the four-point functions of the component fields, we have the possibility to write this without the differential operator: To get eq. (5.3.78) we have seen, that the application of the differential operator to the function $f\left(\mathcal{I}_{6}{ }^{-1}, \mathcal{I}_{2}\right)$ is equal to the function $f\left(\mathcal{I}_{6}^{\prime-1}, \mathcal{I}_{2}\right)$ with $\mathcal{I}_{6}^{\prime-1}$ from eq. 55.3.79). As this is only caused by rationality of the function $f$ the same has to be true for each partial wave, which can also be expanded as a power series. Hence we have

$$
\left\langle\bar{\Phi}_{1} \bar{\Phi}_{2} \Phi_{3} \Phi_{4}\right\rangle=\frac{1}{x_{\overline{1} 3}{ }^{2\left(\eta_{3}-\eta_{2}\right)} x_{\overline{1} 4}{ }^{2 \eta_{4}} x_{\overline{2} 3}{ }^{2 \eta_{2}}} \sum_{k, L} B_{k, L} \beta_{k L}\left(\mathcal{I}_{6}^{\prime-1}, \mathcal{I}_{2}\right) \text {. }
$$

Thus we have a partial wave expansion of the superconformal four-point function.

A chiral or anti-chiral superfield representation is determined by the representation of their lowest component conformal field, because in contrast to the general case the R-charge is given by the scaling dimension. This suggest the assumption, that we have a more general analogy of the conformal and the chiral/anti-chiral superconformal situation. For the superconformal Casimir operator, $\mathcal{C}_{\text {suco. }}$, analogous to $\mathcal{C}$ in (2.3.4), we then expect, that irreducible positive energy representations of the superconformal group on chiral superspace, i.e. chiral superfields, are eigenvectors. The corresponding differential operator, $\mathcal{C}_{\text {suco., } \mathcal{I}_{6}{ }^{-1}, \mathcal{I}_{2}}$, acting on the $f\left(\mathcal{I}_{6}{ }^{-1}, \mathcal{I}_{2}\right)$ should then have the superconformal partial waves, $\beta_{k L}\left(\mathcal{I}_{6}^{\prime-1}, \mathcal{I}_{2}\right)$, as eigenfunctions. The 
partial waves being the same functions the differential operator, $\mathcal{C}_{\text {suco. }} \mathcal{I}_{6}{ }^{-1} \mathcal{I}_{2}$, has to be equal to the conformal one, $\mathcal{C}_{s, t}$, with $s$ and $t$ replaced by $\mathcal{I}_{6}{ }^{-1}$ and $\mathcal{I}_{2}$, as it does not matter, which name the variables in the equation for the eigenvalues have.

If one has calculated for a concrete case the coefficients of the partial wave expansion, $B_{k, L}$, one can then also analyze the restrictions on the amplitudes, on which these coefficients depend, coming from positivity. As the partial waves are shown to be all positive from free field calculations (cf. [NRT05]), all coefficients have to be separatly positive.

The coefficients, $B_{k, L}$, here in this superconformal four-point function are the same as the ones in the conformal scalar four-point function. But the other four-point functions of the higher component fields have also partial wave expansions. After we have seen, that the pole bounds from different component four-point functions have different results on the superconformal four-point function, the question is, if also the different partial wave expansions have different results on the superconformal level and thus positivity of each one restricts the amplitudes in a different way leading to altogether stronger restrictions.

To check this one has to work out partial wave expansions of four-point functions with less identical fields, weaker pole bounds due to $\phi_{i} \neq \phi_{j}{ }^{*}$, with fields with higher scaling dimensions and with fermionic fields. These correlation functions have more amplitudes, which are independent in the conformal case. But these amplitudes have to be related by their role as component correlation functions in the superior structure.

This approach would be rather tedious, but eventually it is not needed. We have already gotten a large simplification due to the pole bounds of higher component fields in comparison to the conformal case with the same identities from locality.

But the transfer of the conformal partial wave expansion for scalar lowest component fields also means a transfer of the conditions from positivity on the partial wave coefficients in consideration of eq. 6.2.5). Then one can also ask the following question on the other direction: Can we extract information on the positivity of the other component correlation functions from the correlation function of superfields? This could lead to a significant simplification compared to the calculation of the partial wave expansions of all four-point functions of component field. 


\section{Chapter 7}

\section{Conclusion}

This thesis builds a basis for further investigations of $\mathcal{N}=1$ superconformal invariance in four dimensions. Most of the results are valid for general superconformal field theories, but we also emphasized at specific points the specialties of global superconformal symmetry.

The investigation of scalar invariants has taken place under the most general conditions in the main text for partial and full three point invariants and full fourpoint invariants and in the appendix for partial four-point invariants.

Three and four-point functions with vanishing R-charge of general scalar superfields can be expressed with ten invariants. Two are non-nilpotent and eight are nilpotent. This is a significant reduction to the former knowledge, that counted 16 invariants for $\mathcal{N}=1$.

We have also seen, that there will not be a further reduction of the number of invariants, as none of these ten invariants can be expressed by the others. Even more we have listed all independent monomial of the non-nilpotent invariants, which by definition cannot be expressed by a linear combination of the other nilpotent monomials with coefficients, which are functions of non-nilpotent invariants. This led to an expansion of any four-point invariant in these nilpotent invariants with coefficient functions depending on two non-nilpotent invariants, which is unique after the choice of the ten invariants is fixed.

In the context of these expansions this thesis contains several sections describing computational methods and in the appendix procedures for Maple, which are useful for the calculations with four-point invariants.

The chosen form of the chiral/anti-chiral scalar four-point function and the proof, which leads to it from the general scalar four-point function, are not only useful in the context of global superconformal field theories, but more generally starting points for further investigations. First it appears novel, that a superconformal four-point function is essentially expressed as the conformal four-point function of the lowest component fields evaluated at superconformal instead of conformal cross ratios, on which a differential operator is applied. Second the calculations are given in a form, which makes it easy to see, how the chiral and anti-chiral restrictions act and lead to the proven form of their four-point function. One can, for example directly see, that there are only three of four restrictions used in the lowest order calculations of the 
proof of theorem 5.1 and the fourth is then fulfilled automatically (cf. eq. (5.3.27)). This already means in combination with the iteration condition used afterwards for the higher orders, that a four-point function with two chiral, only one anti-chiral - or the other way around - superfield and a more general fourth superfield has a solution also fixed by the lowest component fields. However, it remains to be checked, if or if not this is also the most general solution to the restrictions.

The mentioned differential operator results from a simple equation, which relates terms of different orders of nilpotent invariants in the four-point function for all chiral and anti-chiral restrictions of the four-point function simultaneously. Eq. 5.3 .45 is valid for all four derivatives with respect to Graßmann variables from these restrictions. It has on the one side the derivative of a nilpotent invariant, which lowers the order in Graßmann variables of the term by eliminating one of these variables. On the other side the derivative acts on a non-nilpotent invariants, which produces a nilpotent factor and raises the order in Graßmann variables. Thus the two terms originating from different orders in the four-point function meet in the middle, when the derivative is applied to it. Such equations are central in order to determine the dependences between different orders or equivalently different component fields within correlation functions of superfields .

In the last chapter we have started the investigation of global superconformal symmetry within the framework of constructive/axiomatic field theory. The aim is to find a model, which fulfills the Wightman axioms, especially the positivity of all correlation functions and Schwarz inequalities from mixed terms. The aim is to show, that it is an interacting model. This is a tremendous task, if one looks at the problems in the discussion of the four-point functions, and sees, that this is only a first step. Global superconformal models add more possibilities to the global conformal models, for which partial wave analysis can be used for this task. Due to the supersymmetry relating different component fields in a multiplet the superconformal models are subject to different combinations of pole bounds than global conformal models. One can apply the partial wave analysis to correlation function restricted by these pole bounds and gets different expansions and different conditions from positivity on the coefficients in these expansions. If positivity conditions leave for a global conformal model only the possibility, that it is free, a similarly complicated superconformal model might - in a logic sequence - be the next case to investigate, before moving on to more complicated global conformal models.

With supersymmetry research in axiomatic field theory spreads in a new direction in order to broaden its view on structures in quantum field theory. 


\section{Appendix A}

\section{Conventions and useful equations}

The conventions in this thesis are those of [Par99]. The Minkoski metric is $\eta^{\mu \nu}=$ $\operatorname{diag}(+1,-1,-1,-1)$.

\section{A.1 Pauli matrices}

The Pauli matrices $\sigma_{\alpha \dot{\alpha}}^{\mu}$ are given by

$$
\begin{aligned}
\sigma^{0} & =\left(\begin{array}{ll}
1 & 0 \\
0 & 1
\end{array}\right), \\
\sigma^{1} & =\left(\begin{array}{ll}
0 & 1 \\
1 & 0
\end{array}\right), \\
\sigma^{2} & =\left(\begin{array}{cc}
0 & -\mathrm{i} \\
\mathrm{i} & 0
\end{array}\right), \\
\sigma^{3} & =\left(\begin{array}{cc}
1 & 0 \\
0 & -1
\end{array}\right) .
\end{aligned}
$$

We can raise the spinoral indices with anti-symmetric matrices $\left(\epsilon^{-1}\right)^{\beta \alpha}$ and $\left(\bar{\epsilon}^{-1}\right)^{\dot{\beta} \dot{\alpha}}$ and lower them with their inverses $\epsilon_{\alpha \beta}$ and $\bar{\epsilon}_{\dot{\alpha} \dot{\beta}}$, where we have $\epsilon_{12}=\bar{\epsilon}_{\dot{1} \dot{2}}=\left(\epsilon^{-1}\right)^{21}=$ $\left(\bar{\epsilon}^{-1}\right)^{\dot{2} \mathrm{i}}=1$. One also finds for the inverses,

$$
\left(\epsilon^{-1}\right)^{\beta \alpha}=-\epsilon^{\beta \alpha}, \quad\left(\bar{\epsilon}^{-1}\right)^{\dot{\beta} \dot{\alpha}}=-\bar{\epsilon}^{\dot{\beta} \dot{\alpha}} .
$$

For the Pauli matrices we use the notation,

$$
\left(\bar{\sigma}^{\mu}\right)^{\dot{\beta} \beta}=\left(\epsilon^{-1}\right)^{\beta \alpha}\left(\bar{\epsilon}^{-1}\right)^{\dot{\beta} \dot{\alpha}} \sigma_{\alpha \dot{\alpha}}^{\mu},
$$

and also have the relation,

$$
\epsilon\left(\bar{\sigma}^{\mu}\right)^{t} \bar{\epsilon}=-\sigma^{\mu} .
$$


A Pauli matrix contracted with another one is

$$
\left(\sigma^{\mu}\right)_{\alpha \dot{\alpha}}\left(\sigma_{\mu}\right)_{\beta \dot{\beta}}=2 \epsilon_{\alpha \beta} \bar{\epsilon}_{\dot{\alpha} \dot{\beta}}
$$

From the relation of Pauli matrices,

$$
\sigma_{\mu} \bar{\sigma}_{\nu}+\sigma_{\nu} \bar{\sigma}_{\mu}=2 \eta_{\mu \nu}
$$

we get a the following useful equation

$$
\sigma_{\rho}\left(\bar{\sigma}_{\mu} \sigma_{\nu}-\bar{\sigma}_{\nu} \sigma_{\mu}\right)=\left(\sigma_{\mu} \bar{\sigma}_{\nu}-\sigma_{\nu} \bar{\sigma}_{\mu}\right) \sigma_{\rho}+4 \eta_{\mu \rho} \sigma_{\nu}-4 \eta_{\nu \rho} \sigma_{\mu}
$$

which fits the frequent situation, in which the Pauli matrix $\sigma_{\rho}$ has to "brought to the other side" of the antisymmetrization of Pauli matrices. The trace of a product of two Pauli matrices is

$$
\operatorname{tr}\left(\sigma_{\mu} \bar{\sigma}_{\nu}\right)=2 \eta_{\mu \nu}
$$

For four Pauli matrices we get,

$$
\frac{1}{2} \operatorname{tr}\left(\sigma_{\mu} \bar{\sigma}_{\nu} \sigma_{\rho} \bar{\sigma}_{\lambda}\right)=\eta_{\mu \nu} \eta_{\rho \lambda}+\eta_{\mu \lambda} \eta_{\nu \rho}-\eta_{\mu \rho} \eta_{\nu \lambda}+\mathrm{i} \epsilon_{\mu \nu \rho \lambda}
$$

Products of two Pauli matrices multiplied by the matrix $\epsilon$ resp. $\epsilon^{-1}$ have the following properties,

$$
\begin{aligned}
&\left(\sigma_{\mu}\right)_{\alpha \dot{\alpha}}\left(\bar{\sigma}_{\nu}\right)^{\dot{\alpha} \gamma} \epsilon_{\gamma \beta}-\left(\sigma_{\nu}\right)_{\beta \dot{\alpha}}\left(\bar{\sigma}_{\mu}\right)^{\dot{\alpha} \gamma} \epsilon_{\gamma \alpha}, \\
&\left(\epsilon^{-1}\right)^{\alpha \gamma}\left(\sigma_{\mu}\right)_{\gamma \dot{\alpha}}\left(\bar{\sigma}_{\nu}\right)^{\dot{\alpha} \beta}=-\left(\epsilon^{-1}\right)^{\beta \gamma}\left(\sigma_{\nu}\right)_{\gamma \dot{\alpha}}\left(\bar{\sigma}_{\mu}\right)^{\dot{\alpha} \alpha} .
\end{aligned}
$$

It follows, that the antisymmetrization with respect to the four-vector indices is symmetric in the spinoral indices. We have

$$
\left(\sigma_{\mu} \bar{\sigma}_{\nu}-\sigma_{\nu} \bar{\sigma}_{\mu}\right)_{\alpha}^{\gamma} \epsilon_{\gamma \beta}=\left(\sigma_{\mu} \bar{\sigma}_{\nu}-\sigma_{\nu} \bar{\sigma}_{\mu}\right)_{\beta}{ }^{\gamma} \epsilon_{\gamma \alpha}
$$

and the corresponding equation with $\epsilon^{-1}$ multiplied from the other side.

The same can, of course, be done also with the product of Pauli matrices turned around:

$$
\begin{aligned}
\left(\bar{\sigma}_{\mu}\right)^{\dot{\alpha} \alpha}\left(\sigma_{\nu}\right)_{\alpha \dot{\gamma}}\left(\bar{\epsilon}^{-1}\right)^{\dot{\gamma} \dot{\beta}}=-\left(\bar{\sigma}_{\nu}\right)^{\dot{\beta} \alpha}\left(\sigma_{\mu}\right)_{\alpha \dot{\gamma}}\left(\bar{\epsilon}^{-1}\right)^{\dot{\gamma} \dot{\alpha}} \\
\left(\bar{\sigma}_{\mu} \sigma_{\nu}-\bar{\sigma}_{\nu} \sigma_{\mu}\right)^{\dot{\alpha}}{ }_{\dot{\gamma}}\left(\bar{\epsilon}^{-1}\right)^{\dot{\gamma} \dot{\beta}}=\left(\bar{\sigma}_{\mu} \sigma_{\nu}-\bar{\sigma}_{\nu} \sigma_{\mu}\right)_{\dot{\gamma}}^{\dot{\beta}}\left(\bar{\epsilon}^{-1}\right)^{\dot{\gamma} \dot{\alpha}} .
\end{aligned}
$$

Also here the analogous equations with $\bar{\epsilon}$ multiplied from the other side are true. Any vector $v^{\mu}$ can be written as $2 \times 2$ hermitian matrices by the contractions with Pauli matrices,

$$
\begin{aligned}
\mathrm{v}_{\alpha \dot{\alpha}} & =v^{\mu} \sigma_{\mu_{\alpha \dot{\alpha}}} \\
\tilde{\mathrm{v}}^{\dot{\alpha} \alpha} & =v^{\mu} \bar{\sigma}_{\mu}^{\dot{\alpha} \alpha} .
\end{aligned}
$$




\section{A.2 Superconformal intervals}

If we contract the superconformal interval, $x_{\bar{i} j}{ }^{\mu}$ in these ways, the product is,

$$
\mathrm{x}_{i j}^{-} \tilde{\mathrm{x}}_{i j}=x_{i j}^{2} 1_{2 \times 2} .
$$

Different intervals are related by

$$
\begin{gathered}
\mathrm{x}_{\bar{i} k}=\mathrm{x}_{\bar{i} j}+\mathrm{x}_{\bar{j} k}+4 \mathrm{i} \tilde{\theta}_{j k} \tilde{\bar{\theta}}_{i j}, \\
\tilde{\mathrm{x}}_{i k}=\tilde{\mathrm{x}}_{\bar{i} j}+\tilde{\mathrm{x}}_{\bar{j} k}-4 \mathrm{i} \bar{\theta}_{i j} \theta_{j k} .
\end{gathered}
$$

We can switch the indices with the help of

$$
\mathrm{x}_{i j}=-\mathrm{x}_{\overline{j i}}+4 \mathrm{i} \tilde{\theta}_{j i} \tilde{\bar{\theta}}_{j i} .
$$

Written with four-vectors this is

$$
x_{\overline{i j}}{ }^{\mu}=-x_{j i}{ }^{\mu}+2 \mathrm{i} \theta_{j i} \sigma^{\mu} \bar{\theta}_{j i} .
$$

Products of spinoral intervals, $\theta_{i j}$ and $\bar{\theta}_{i j}$, can often be simplified with the help of eqns. 3.2.12 and (3.2.13). With these equations one can show for these intervals,

$$
\begin{aligned}
\theta_{i j} \sigma^{\mu} \bar{\theta}_{i j} \theta_{i j} \sigma^{\nu} \bar{\theta}_{i j} & =\frac{1}{2} \eta^{\mu \nu} \theta_{i j} \tilde{\theta}_{i j} \tilde{\tilde{\theta}}_{i j} \bar{\theta}_{i j}, \\
\theta_{i j} \sigma^{\mu} \bar{\theta}_{i j} \theta_{i j} \sigma_{\mu} \bar{\theta}_{i j} & =\frac{4}{y^{2}}\left(\theta_{i j} \bar{y}_{i j}\right),
\end{aligned}
$$

where $y^{\mu}$ may be any vector or vector-valued function with invertable square. The analogues equations are also true for other spinors, which fulfill equations (3.2.12) and 3.2 .13 , such as $\Theta_{1(j)}$ and $\bar{\Theta}_{1(j)}$ (cf. eqns. 4.3.21) and 4.3.22).

We frequently use the abbreviation

$$
\rho_{i j}=\theta_{i j} \tilde{x}_{\overline{i j}}^{-1} \bar{\theta}_{i j}
$$

such as in the following case. The square of eq. A.2.5 can be simplified as

$$
\begin{aligned}
{x_{\bar{i} j}}^{2} & =x_{\overline{j i}}{ }^{2}-4 \mathrm{i} x_{\bar{j}}{ }^{\mu} \theta_{j i} \sigma_{\mu} \bar{\theta}_{j i}-4 \theta_{j i} \sigma^{\mu} \bar{\theta}_{j i} \theta_{j i} \sigma_{\mu} \bar{\theta}_{j i} \\
& =x_{\bar{j} i}{ }^{2}\left(1-4 \mathrm{i} \rho_{j i}-16 \rho_{j i}{ }^{2}\right),
\end{aligned}
$$

using eq. A.2.7) for the second line.

Let a variable or function $y=y_{0}+y_{1}$ consist of a non-nilpotent part $y_{0}$ and a nilpotent part $y_{1}$ containing Graßmann spinors, $\theta$ and $\bar{\theta}$. If the nilpotent part has an overall Graßmann factor, $y_{1}=\theta(\ldots)$ or $y_{1}=(\ldots) \bar{\theta}$, its third power already vanishes. So its inverse can be calculated by an expansion around $y_{1}=0$, which ends after the second order term:

$$
\frac{1}{y}=\frac{1}{y_{0}}-\frac{1}{y_{0}^{2}} y_{1}+\frac{1}{y_{0}^{3}} y_{1}^{2} .
$$


This can be used to calculate

$$
\frac{1}{x_{i j}^{2}}=\frac{1}{x_{\bar{j} i}{ }^{2}}\left(1+4 \mathrm{i} \rho_{j i}\right) .
$$

The squares of $\rho_{i j}$ appearing in the first and second order term of the expansion cancel. With the help of eqns. A.2.4, A.2.6, A.2.7) and A.2.11 we find

$$
\rho_{i j}=-\rho_{j i}+4 \mathrm{i} \rho_{j i}{ }^{2} .
$$




\section{Appendix B}

\section{$\mathcal{N}=1$ superconformal algebra}

In section 3.3 we have studied the different superconformal transformations. In eqns. (3.3.2) through 3.3.20 we encountered all parameters defining the specific transformations. Here we chose the split into dilations and R-symmetry (eqns. (3.3.15)(3.3.20 ) rather than the expression in terms of superdilations (eqns. (3.3.5)-(3.3.5). This way any superconformal transformation is given by the set of parameters, $\left(\left(a^{\mu}, \alpha, \bar{\alpha}\right), \lambda, \Omega, w^{\mu \nu},\left(b^{\mu}, \beta, \bar{\beta}\right)\right) \in \mathbb{R}^{4 \mid 4} \times \mathbb{R}^{+} \times \mathbb{R} \times \mathbb{R}^{6} \times \mathbb{R}^{4 \mid 4}$, These belong to translations of superspace or supertranslations, dilations, R-symmetry transformations, Lorentz transformations and special superconformal transformations, respectively. The corresponding generators are $\left(P_{\mu}, Q, \bar{Q}, D, R, M_{\mu \nu}, K_{\mu}, S, \bar{S}\right)$.

From two successively executed infinitesimal superconformal transformations (cf. eq. (3.3.26) $)$ the superconformal algebra, these generators fulfill, can be deduced. The $\mathcal{N}$ extended algebra is given in the appendix of [Par99]. We first have the super-Poincaré algebra as in section 3.1

$$
\begin{aligned}
{\left[P_{\mu}, P_{\nu}\right] } & =0 \\
{\left[P_{\mu}, Q\right] } & =\left[P_{\mu}, \bar{Q}\right]=\{Q, Q\}=\{\bar{Q}, \bar{Q}\}=0, \\
\{Q, \bar{Q}\} & =2 \sigma^{\mu} P_{\mu}, \\
{\left[M_{\mu \nu}, P_{\rho}\right] } & =\mathrm{i}\left(\eta_{\mu \rho} P_{\nu}-\eta_{\nu \rho} P_{\mu}\right), \\
{\left[M_{\mu \nu}, Q\right] } & =\frac{1}{4} \mathrm{i}\left(\sigma_{\mu} \bar{\sigma}_{\nu}-\sigma_{\nu} \bar{\sigma}_{\mu}\right) Q, \\
{\left[M_{\mu \nu}, \bar{Q}\right] } & =-\frac{1}{4} \mathrm{i} \bar{Q}\left(\bar{\sigma}_{\mu} \sigma_{\nu}-\bar{\sigma}_{\nu} \sigma_{\mu}\right), \\
{\left[M_{\mu \nu}, M_{\rho \sigma}\right] } & =\mathrm{i}\left(\eta_{\mu \rho} M_{\nu \sigma}-\eta_{\mu \sigma} M_{\nu \rho}-\eta_{\nu \rho} M_{\mu \sigma}+\eta_{\nu \sigma} M_{\mu \rho}\right) .
\end{aligned}
$$

Another copy of this algebra is also a subalgebra of the superconformal algebra. It is given by the conjugate subalgebra und superinversion. This special superconformal algebra contains the generators $\left(K_{\mu}, S, \bar{S}, M_{\mu, \nu}\right)$. Of cause, it also contains eq. (B.0.7) and

$$
\begin{aligned}
{\left[K_{\mu}, K_{\nu}\right] } & =0, \\
{\left[K_{\mu}, S\right] } & =\left[K_{\mu}, \bar{S}\right]=\{S, S\}=\{\bar{S}, \bar{S}\}=0, \\
\{S, \bar{S}\} & =2 \sigma^{\mu} K_{\mu},
\end{aligned}
$$




$$
\begin{aligned}
{\left[M_{\mu \nu}, K_{\rho}\right] } & =\mathrm{i}\left(\eta_{\mu \rho} K_{\nu}-\eta_{\nu \rho} K_{\mu}\right), \\
{\left[M_{\mu \nu}, S\right] } & =\frac{1}{4} \mathrm{i}\left(\sigma_{\mu} \bar{\sigma}_{\nu}-\sigma_{\nu} \bar{\sigma}_{\mu}\right) S, \\
{\left[M_{\mu \nu}, \bar{S}\right] } & =-\frac{1}{4} \mathrm{i} \bar{S}\left(\bar{\sigma}_{\mu} \sigma_{\nu}-\bar{\sigma}_{\nu} \sigma_{\mu}\right) .
\end{aligned}
$$

We also find the conformal Lie algebra consisting of all commutators without spinoral generators, which we had so far in eqns. (B.0.1), (B.0.4), (B.0.7), (B.0.8) and B.0.11, and

$$
\begin{aligned}
{\left[P_{\mu}, K_{\nu}\right] } & =2 \mathrm{i}\left(M_{\mu \nu}+\eta_{\mu \nu} D\right) \\
{\left[D, P_{\mu}\right] } & =-\mathrm{i} P_{\mu} \\
{\left[D, K_{\mu}\right] } & =\mathrm{i} K_{\mu} \\
{[D, D] } & =\left[D, M_{\mu \nu}\right]=0
\end{aligned}
$$

The rest of the commutators with the dilation operators are

$$
\begin{aligned}
{[D, Q] } & =-\mathrm{i} \frac{1}{2} Q \\
{[D, \bar{Q}] } & =-\mathrm{i} \frac{1}{2} \bar{Q}, \\
{[D, S] } & =\mathrm{i} \frac{1}{2} S, \\
{[D, \bar{S}] } & =\mathrm{i} \frac{1}{2} \bar{S},
\end{aligned}
$$

We have encountered now all generators except the R-symmetry generator $R$. But this we also find in the missing (anti-)commutators of the other generators:

$$
\begin{aligned}
{\left[P_{\mu}, S\right] } & =\sigma_{\mu} \tilde{\bar{Q}} \\
{\left[P_{\mu}, \bar{S}\right] } & =-\tilde{Q} \sigma_{\mu} \\
{\left[K_{\mu}, Q\right] } & =\sigma_{\mu} \tilde{\bar{S}} \\
{\left[K_{\mu}, \bar{Q}\right] } & =-\tilde{S} \sigma_{\mu} \\
\left\{Q_{\alpha}, S_{\beta}\right\} & =-2 \mathrm{i} \epsilon_{\alpha \beta} D-\frac{1}{2} \mathrm{i}\left(\sigma_{\alpha \dot{\alpha}}^{\mu}\left(\bar{\sigma}^{\nu}\right)^{\dot{\alpha} \gamma}-\sigma_{\alpha \dot{\alpha}}^{\nu}\left(\bar{\sigma}^{\mu}\right)^{\dot{\alpha} \gamma}\right) \epsilon_{\gamma \beta} M_{\mu \nu}+6 \mathrm{i} \epsilon_{\alpha \beta} R, \\
\left\{\bar{Q}_{\dot{\alpha}}, \bar{S}_{\dot{\beta}}\right\} & =2 \mathrm{i} \bar{\epsilon}_{\dot{\alpha} \dot{\beta}} D-\frac{1}{2} \mathrm{i} \bar{\epsilon}_{\dot{\beta} \dot{\gamma}}\left(\left(\bar{\sigma}^{\mu}\right)^{\dot{\gamma} \alpha} \sigma_{\alpha \dot{\alpha}}^{\nu}-\left(\bar{\sigma}^{\nu}\right)^{\dot{\gamma} \alpha} \sigma_{\alpha \dot{\alpha}}^{\mu}\right) M_{\mu \nu}+6 \mathrm{i} \bar{\epsilon}_{\dot{\alpha} \dot{\beta}} R \\
\{Q, \bar{S}\} & =\left\{\bar{Q}_{\bar{Q}}, S\right\}=0 .
\end{aligned}
$$

The prefactor of the R-symmetry operator $R$ in the last two anti-commutators is conventional and leads to the relation of the scaling dimension and the R-charge of chiral and antichiral superfields in eqns. (3.4.59) and (3.4.60). 
Finally the commutators with $R$ are

$$
\begin{aligned}
{[R, Q] } & =-\mathrm{i} \frac{1}{2} Q \\
{[R, \bar{Q}] } & =\mathrm{i} \frac{1}{2} \bar{Q} \\
{[R, S] } & =\mathrm{i} \frac{1}{2} S \\
{[R, \bar{S}] } & =-\mathrm{i} \frac{1}{2} \bar{S} \\
{[R, R] } & =[R, D]=\left[R, P_{\mu}\right]=\left[R, K_{\mu}\right]=\left[R, M_{\mu \nu}\right]=0 .
\end{aligned}
$$

This completes the superconformal algebra consisting of all (anti-)commutators in this chapter. 


\section{Appendix C}

\section{Details on Invariants}

\section{C.1 Lowest order of the nilpotent invariants $I_{i j k}$ and their derivatives}

In this section we provide a list of the lowest order terms of the nilpotent invariants, $I_{i j k}$ and $I_{0}$, from eqns. (4.3.3), 4.3.4) and 4.3.70). They and their derivatives are needed for the proof of theorem 5.1. The lowest order of the invariants are presented here in terms of the lowest order of the supersymmetric intervals (eq. (3.2.18)-3.2.20) for $i, j=1,2,3,4$ and $i \neq j$ :

$$
\mathrm{x}_{i j} \stackrel{\text { l.o. }}{=} \mathrm{x}_{i j}=\mathrm{x}_{i}-\mathrm{x}_{j} \text {. }
$$

For their derivatives we abbreviate with the help of the lowest order of the functions of three points, $\tilde{\mathrm{X}}_{1(1)+}, \tilde{\mathrm{X}}_{1(2)-}, \tilde{\mathrm{X}}_{1+}$ (eqns. 4.2.3, 4.2.4 and 4.1.1)) and the corresponding matrices with lower indices:

$$
\begin{gathered}
\tilde{\mathrm{X}}_{1(1)+} \stackrel{\text { l.o. }}{=} \mathrm{x}_{12}{ }^{-1}-\mathrm{x}_{14}{ }^{-1} \equiv \tilde{\mathrm{X}}_{1(1) 0} \\
\tilde{\mathrm{X}}_{1(2)-} \stackrel{\text { l.o. }}{=} \mathrm{x}_{13}{ }^{-1}-\mathrm{x}_{14}{ }^{-1} \equiv \tilde{\mathrm{X}}_{1(2) 0} \\
\tilde{\mathrm{X}}_{1+} \stackrel{\text { l.o. }}{=} \mathrm{x}_{12}{ }^{-1}-\mathrm{x}_{13}{ }^{-1} \equiv \tilde{\mathrm{X}}_{10} .
\end{gathered}
$$

These expressions do not differ for an index + instead of - and vice versa.

We start with $I_{i j k}$ for $i, j, k=1,2$ :

$$
\begin{aligned}
I_{111} \stackrel{\text { l.o. }}{=} & -\theta_{12} \tilde{\mathrm{x}}_{12}^{-1} \bar{\theta}_{12}-\theta_{24} \tilde{\mathrm{x}}_{24}^{-1} \bar{\theta}_{24}+\theta_{14} \tilde{\mathrm{x}}_{14}^{-1} \bar{\theta}_{14} \\
I_{112} \stackrel{\text { l.o. }}{=} & -\theta_{14} \tilde{\mathrm{x}}_{24}^{-1} \bar{\theta}_{24}+\theta_{14} \tilde{\mathrm{x}}_{14}^{-1} \bar{\theta}_{14}-\theta_{13} \tilde{\mathrm{x}}_{13}^{-1} \bar{\theta}_{12}+\theta_{13} \tilde{\mathrm{x}}_{13}^{-1} \tilde{\mathrm{x}}_{12} \tilde{\mathrm{x}}_{24}^{-1} \bar{\theta}_{24} \\
I_{121} \stackrel{\text { l.o. }}{=} & -\theta_{24} \tilde{\mathrm{x}}_{24}^{-1} \bar{\theta}_{34}-\theta_{12} \tilde{\mathrm{x}}_{13}^{-1} \bar{\theta}_{13}+\theta_{14} \tilde{\mathrm{x}}_{14}^{-1} \bar{\theta}_{14}-\theta_{24} \tilde{\mathrm{x}}_{24}^{-1} \tilde{\mathrm{x}}_{23} \tilde{\mathrm{x}}_{13}^{-1} \bar{\theta}_{13} \\
I_{122} \stackrel{\text { l.o. }}{=} & -\frac{x_{12} x_{34}^{2}}{x_{13} x_{24}^{2}}\left(\theta_{34} \tilde{\mathrm{x}}_{34}^{-1} \bar{\theta}_{34}+\theta_{14} \tilde{\mathrm{x}}_{34}^{-1} \bar{\theta}_{14}+\theta_{13} \tilde{\mathrm{x}}_{13}^{-1} \bar{\theta}_{13}\right) \\
& +\frac{x_{14}{ }^{2} x_{23}{ }^{2}}{x_{13}{ }^{2} x_{24}{ }^{2}}\left(\theta_{34} \tilde{\mathrm{x}}_{23}^{-1} \bar{\theta}_{13}+\theta_{13} \tilde{\mathrm{x}}_{13}^{-1} \bar{\theta}_{13}\right) \\
& -\frac{1}{x_{13} x_{24}{ }^{2}}\left(\theta_{14} \mathrm{x}_{14} \tilde{\mathrm{x}}_{24} \mathrm{x}_{23} \bar{\theta}_{13}-\theta_{13} \mathrm{x}_{23} \tilde{\mathrm{x}}_{12} \mathrm{x}_{14} \bar{\theta}_{14}\right)
\end{aligned}
$$




$$
\begin{aligned}
& I_{211} \stackrel{\text { l.o. }}{=}-\theta_{24} \tilde{\mathrm{x}}_{24}^{-1} \bar{\theta}_{24}-\theta_{14} \tilde{\mathrm{x}}_{24}^{-1} \bar{\theta}_{14}-\theta_{12} \tilde{\mathrm{x}}_{12}^{-1} \bar{\theta}_{12} \\
& -\frac{x_{14}^{2} x_{23}^{2}}{x_{13}{ }^{2} x_{24}{ }^{2}}\left(\theta_{24} \tilde{\mathrm{x}}_{23}^{-1} \bar{\theta}_{12}-\theta_{12} \tilde{\mathrm{x}}_{12}^{-1} \bar{\theta}_{12}\right) \\
& +\frac{1}{x_{13}{ }^{2} x_{24}{ }^{2}}\left(\theta_{14} \mathrm{x}_{14} \tilde{\mathrm{x}}_{34} \mathrm{x}_{23} \bar{\theta}_{12}-\theta_{12} \mathrm{x}_{23} \tilde{\mathrm{x}}_{13} \mathrm{x}_{14} \bar{\theta}_{14}\right), \\
& I_{212} \stackrel{\text { l.o. }}{=}-\frac{x_{12}^{2} x_{34}^{2}}{x_{13}{ }^{2} x_{24}{ }^{2}}\left(\theta_{34} \tilde{\mathrm{x}}_{34}^{-1} \bar{\theta}_{24}+\theta_{13} \tilde{\mathrm{x}}_{12}^{-1} \bar{\theta}_{12}-\theta_{14} \tilde{\mathrm{x}}_{14}^{-1} \bar{\theta}_{14}\right) \\
& +\frac{1}{x_{13}^{2} x_{24}^{2}} \theta_{34} \mathrm{x}_{34} \tilde{\mathrm{x}}_{23} \mathrm{x}_{12} \bar{\theta}_{12} \text {, } \\
& I_{221} \stackrel{\text { l.o. }}{=}-\frac{x_{12}^{2} x_{34}^{2}}{x_{13}{ }^{2} x_{24}{ }^{2}}\left(\theta_{14} \tilde{\mathrm{x}}_{34}^{-1} \bar{\theta}_{34}-\theta_{14} \tilde{\mathrm{x}}_{14}^{-1} \bar{\theta}_{14}+\theta_{12} \tilde{\mathrm{x}}_{12}^{-1} \bar{\theta}_{13}\right) \\
& +\frac{1}{x_{13}^{2} x_{24}^{2}} \theta_{12} \mathrm{x}_{12} \tilde{\mathrm{x}}_{13} \mathrm{x}_{34} \bar{\theta}_{34} \text {, } \\
& I_{222} \stackrel{\text { l.o. }}{=}-\frac{x_{12}^{2} x_{43}^{2}}{x_{13}{ }^{2} x_{42}{ }^{2}}\left(\theta_{13} \tilde{\mathrm{x}}_{13}^{-1} \bar{\theta}_{13}+\theta_{34} \tilde{\mathrm{x}}_{34}^{-1} \bar{\theta}_{34}-\theta_{14} \tilde{\mathrm{x}}_{14}^{-1} \bar{\theta}_{14}\right) \text {. }
\end{aligned}
$$

At last the combination $I_{0}$ of these invariants, which is essentially the three point invariant times cross ratios (cf. eq. 4.3.69), is given by

$$
I_{0}=\frac{x_{14}^{2} x_{23}^{2}}{x_{13}{ }^{2} x_{24}{ }^{2}}\left(-\theta_{12} \tilde{\mathrm{x}}_{12}^{-1} \bar{\theta}_{12}-\theta_{23} \tilde{\mathrm{x}}_{23}^{-1} \bar{\theta}_{23}+\theta_{13} \tilde{\mathrm{x}}_{13}^{-1} \bar{\theta}_{13}\right) .
$$

Their derivatives are

$$
\begin{aligned}
& D_{1-} I_{i j k} \stackrel{\text { l.o. }}{=}-\mathrm{i} \frac{\mathrm{X}_{1(k) 0} \tilde{\mathrm{X}}_{1(i) 0}}{\mathrm{X}_{1(1) 0}{ }^{2}} \tilde{\Theta}_{1(j)}, \\
& D_{2-} I_{i j 2} \stackrel{\text { l.o. }}{=} 0 \text {, } \\
& D_{2-} I_{i j 1} \stackrel{\text { l.o. }}{=} \quad \mathrm{i} \tilde{\mathrm{x}}_{12}^{-1} \frac{\tilde{\mathrm{X}}_{1(i) 0}}{\mathrm{X}_{1(1) 0}{ }^{2}} \tilde{\Theta}_{1(j)} \text {, } \\
& \bar{D}_{3+} I_{i 1 k} \stackrel{\text { l.o. }}{=} 0 \text {, } \\
& \bar{D}_{3+} I_{i 2 k} \stackrel{\text { l.o. }}{=} \quad \mathrm{i} \tilde{\Theta}_{1(k)} \frac{\mathrm{X}_{1(i) 0}}{\mathrm{X}_{1(1) 0}{ }^{2}} \mathrm{x}_{13}{ }^{-1} \text {, } \\
& \bar{D}_{4+} I_{i j k} \stackrel{\text { l.o. }}{=}-\mathrm{i} \tilde{\bar{\Theta}}_{1(k)} \frac{\mathrm{X}_{1(i) 0}}{\mathrm{X}_{1(1) 0}{ }^{2}} \mathrm{x}_{14}{ }^{-1} \text {, } \\
& D_{1-} I_{\Sigma} \stackrel{\text { l.o. }}{=}-\mathrm{i} \frac{\mathrm{X}_{10} \tilde{\mathrm{X}}_{10}}{\mathrm{X}_{1(1) 0}{ }^{2}} \tilde{\Theta}_{1}, \\
& D_{2-} I_{\Sigma} \stackrel{\text { l.o. }}{=} \mathrm{i} \tilde{\mathrm{x}}_{12}^{-1} \frac{\tilde{\mathrm{X}}_{10}}{\mathrm{X}_{1(1) 0}{ }^{2}} \tilde{\Theta}_{1} \\
& \bar{D}_{3+} I_{\Sigma} \stackrel{\text { l.o. }}{=}-\mathrm{i} \tilde{\bar{\Theta}}_{1} \frac{\mathrm{X}_{10}}{\mathrm{X}_{1(1) 0}{ }^{2}} \mathrm{x}_{13}{ }^{-1}, \\
& \bar{D}_{4+} I_{\Sigma} \stackrel{\text { l.o. }}{=} 0
\end{aligned}
$$


where the index 0 denotes the zeroth order in Graßmann variables of the respective variable and $i, j, k=1,2$.

\section{C.2 Derivatives of the nilpotent invariants $T_{111}, T_{212}, T_{222}$ and $T_{0}$}

The derivatives of $T_{111}, T_{222}$ and $T_{0}$ in eqns. 4.3.144, (4.3.147) and 4.3.148), respectively, with respect to the Graßmann variables can be easily calculated, because with eq. A.2.12 one can always convert it into an expression, in which the derivative only acts on the intervals $\theta_{i j}$ resp. $\bar{\theta}_{i j}$ in the appearing $\rho_{i j}$ 's (cf. eq. 4.3.9)). The cause is the dependence of the supersymmetric intervals, $\mathrm{x}_{i j}$, only on the (anti)chiral variables $z_{i-}$ and $z_{j+}$ (cf. eq. (3.2.18)). For example, $T_{111}$ can be brought into the following convenient forms

$$
\begin{aligned}
T_{111} & =\rho_{14}-\rho_{12}-\rho_{24}+2 \mathrm{i}\left(-\rho_{14}{ }^{2}+\rho_{12}{ }^{2}+\rho_{24}{ }^{2}\right) \\
& =\rho_{14}+\rho_{21}-\rho_{24}+2 \mathrm{i}\left(-\rho_{14}{ }^{2}-\rho_{21}{ }^{2}+\rho_{24}{ }^{2}\right) .
\end{aligned}
$$

The first line then directly gives the derivatives with respect to $\theta_{1}$, while the second line is used for the one with repect to $\theta_{2}$. Both are useful for $\bar{\theta}_{4}$ :

$$
\begin{aligned}
\frac{\partial}{\partial \theta_{1}} T_{111} & =\tilde{\mathrm{x}}_{\overline{1} 4}^{-1} \bar{\theta}_{14}-\tilde{\mathrm{x}}_{\overline{1} 2}^{-1} \bar{\theta}_{12}+4 \mathrm{i}\left(-\tilde{\mathrm{x}}_{\overline{1} 4}^{-1} \bar{\theta}_{14} \rho_{14}+\tilde{\mathrm{x}}_{\overline{1} 2}^{-1} \bar{\theta}_{12} \rho_{12}\right) \\
\frac{\partial}{\partial \theta_{2}} T_{111} & =\tilde{\mathrm{x}}_{\overline{1} 4}^{-1} \bar{\theta}_{14}-\tilde{\mathrm{x}}_{\overline{1} 2}^{-1} \bar{\theta}_{12}+4 \mathrm{i}\left(-\tilde{\mathrm{x}}_{\overline{1} 4}^{-1} \bar{\theta}_{14} \rho_{14}+\tilde{\mathrm{x}}_{\overline{1} 2}^{-1} \bar{\theta}_{12} \rho_{12}\right) \\
\frac{\partial}{\partial \bar{\theta}_{3}} T_{111} & =0 \\
\frac{\partial}{\partial \bar{\theta}_{4}} T_{111} & =\tilde{\mathrm{x}}_{\overline{1} 4}^{-1} \bar{\theta}_{14}-\tilde{\mathrm{x}}_{\overline{2} 4}^{-1} \bar{\theta}_{24}+4 \mathrm{i}\left(-\tilde{\mathrm{x}}_{\overline{1} 4}^{-1} \bar{\theta}_{14} \rho_{14}+\tilde{\mathrm{x}}_{\overline{2} 4}^{-1} \bar{\theta}_{24} \rho_{24}\right)
\end{aligned}
$$

Analogously we get the results for $T_{222}$,

$$
\begin{aligned}
\frac{\partial}{\partial \theta_{1}} T_{222} & =\tilde{\mathrm{x}}_{\overline{1} 4}^{-1} \bar{\theta}_{14}-\tilde{\mathrm{x}}_{\overline{1} 3}^{-1} \bar{\theta}_{13}+4 \mathrm{i}\left(-\tilde{\mathrm{x}}_{\overline{1} 4}^{-1} \bar{\theta}_{14} \rho_{14}+\tilde{\mathrm{x}}_{\overline{1} 3}^{-1} \bar{\theta}_{13} \rho_{13}\right) \\
\frac{\partial}{\partial \theta_{2}} T_{222} & =0 \\
\frac{\partial}{\partial \bar{\theta}_{3}} T_{222} & =\tilde{\mathrm{x}}_{\overline{4} 3}^{-1} \bar{\theta}_{43}-\tilde{\mathrm{x}}_{\overline{1} 3}^{-1} \bar{\theta}_{13}+4 \mathrm{i}\left(-\tilde{\mathrm{x}}_{\overline{4} 3}^{-1} \bar{\theta}_{43} \rho_{43}+\tilde{\mathrm{x}}_{\overline{1} 3}^{-1} \bar{\theta}_{13} \rho_{13}\right) \\
\frac{\partial}{\partial \bar{\theta}_{4}} T_{222} & =\tilde{\mathrm{x}}_{\overline{1} 4}^{-1} \bar{\theta}_{14}-\tilde{\mathrm{x}}_{\overline{3} 4}^{-1} \bar{\theta}_{34}+4 \mathrm{i}\left(-\tilde{\mathrm{x}}_{\overline{1} 4}^{-1} \bar{\theta}_{14} \rho_{14}+\tilde{\mathrm{x}}_{\overline{3} 4}^{-1} \bar{\theta}_{34} \rho_{34}\right)
\end{aligned}
$$


and for $T_{0}$,

$$
\begin{aligned}
\frac{\partial}{\partial \theta_{1}} T_{0} & =\tilde{\mathrm{x}}_{\overline{1} 3}^{-1} \bar{\theta}_{13}-\tilde{\mathrm{x}}_{\overline{1} 2}^{-1} \bar{\theta}_{12}+4 \mathrm{i}\left(-\tilde{\mathrm{x}}_{\overline{1} 3}^{-1} \bar{\theta}_{13} \rho_{13}+\tilde{\mathrm{x}}_{\overline{1} 2}^{-1} \bar{\theta}_{12} \rho_{12}\right), \\
\frac{\partial}{\partial \theta_{2}} T_{0} & =\tilde{\mathrm{x}}_{\overline{1} 3}^{-1} \bar{\theta}_{13}-\tilde{\mathrm{x}}_{\overline{1} 2}^{-1} \bar{\theta}_{12}+4 \mathrm{i}\left(-\tilde{\mathrm{x}}_{\overline{1} 3}^{-1} \bar{\theta}_{13} \rho_{13}+\tilde{\mathrm{x}}_{\overline{1} 2}^{-1} \bar{\theta}_{12} \rho_{12}\right), \\
\frac{\partial}{\partial \bar{\theta}_{3}} T_{0} & =\tilde{\mathrm{x}}_{\overline{1} 3}^{-1} \bar{\theta}_{13}-\tilde{\mathrm{x}}_{\overline{2} 3}^{-1} \bar{\theta}_{23}+4 \mathrm{i}\left(-\tilde{\mathrm{x}}_{\overline{1} 3}^{-1} \bar{\theta}_{13} \rho_{13}+\tilde{\mathrm{x}}_{\overline{2} 3}^{-1} \bar{\theta}_{23} \rho_{23}\right), \\
\frac{\partial}{\partial \bar{\theta}_{4}} T_{0} & =0 .
\end{aligned}
$$

Finally we take the derivatives of $T_{212}$ (cf. eq. 4.3.164)):

$$
\begin{aligned}
\frac{\partial}{\partial \theta_{1}} T_{0} & =\tilde{\mathrm{x}}_{\overline{1} 4}^{-1} \bar{\theta}_{14}-\tilde{\mathrm{x}}_{\overline{1} 2}^{-1} \bar{\theta}_{12}+4 \mathrm{i}\left(-\tilde{\mathrm{x}}_{\overline{1} 4}^{-1} \bar{\theta}_{14} \rho_{14}+\tilde{\mathrm{x}}_{\overline{1} 2}^{-1} \bar{\theta}_{12} \rho_{12}\right), \\
\frac{\partial}{\partial \theta_{2}} T_{0} & =0, \\
\frac{\partial}{\partial \bar{\theta}_{3}} T_{0} & =0, \\
\frac{\partial}{\partial \bar{\theta}_{4}} T_{0} & =\tilde{\mathrm{x}}_{\overline{1} 4}^{-1} \bar{\theta}_{14}-\tilde{\mathrm{x}}_{\overline{3} 4}^{-1} \bar{\theta}_{34}+4 \mathrm{i}\left(-\tilde{\mathrm{x}}_{\overline{1} 4}^{-1} \bar{\theta}_{14} \rho_{14}+\tilde{\mathrm{x}}_{\overline{3} 4}^{-1} \bar{\theta}_{34} \rho_{34}\right) .
\end{aligned}
$$

\section{C.3 Products of nilpotent invariants and their deriva- tives}

There are products of nilpotent invariants, $I_{i j k}$, with derivatives of them with respect to the Graßmann variables, which do not vanish, although the product without the derivative is zero because of the nilpotency. An example is (cf. eq. C.1.19)

$$
I_{111}^{2} I_{122} \bar{D}_{4+} I_{112} \text { l.o. }\left(\hat{\tilde{\Theta}}_{1(1)} \hat{\tilde{\mathrm{X}}}_{1(1)+} \hat{\tilde{\Theta}}_{1(1)}\right)^{2} \hat{\tilde{\Theta}}_{1(2)} \hat{\tilde{\mathrm{X}}}_{1(1)+} \hat{\tilde{\Theta}}_{1(2)}\left(\mathrm{i} \tilde{\bar{\Theta}}_{1(2)} \frac{\tilde{\mathrm{X}}_{1(1) 0}}{X_{1(1) 0}{ }^{2}} \mathrm{x}_{14}{ }^{-1}\right)
$$

where the index zero again denotes the zeroth order order in Graßmann variables of the respective variable.

Still for some such monomials it is easy to show, that they vanish for all four derivatives, $\mathfrak{D} \in\left\{D_{1-}, D_{2-}, \bar{D}_{3+}, \bar{D}_{4+}\right\}$. If we take the derivative of $I_{i j k}{ }^{3}$, we immediately get

$$
I_{i j k}^{2} \mathfrak{D} I_{i j k}=0 .
$$

Furthermore the derivative of the vanishing product $I_{i j k}{ }^{2} I_{l j k}$ with $l \neq i$ is

$$
0=2 I_{l j k} I_{i j k} \mathfrak{D} I_{i j k}+I_{i j k}^{2} \mathfrak{D} I_{l j k}
$$

The first summand is zero because of eq. C.3.2, as $I_{l j k} I_{i j k}$ is equal to a nonnilpotent factor times $I_{i j k}{ }^{2}$. 


\section{C.3.1 Proof of Lemma 5.3}

Lemma 5.3 (restated). Let $\alpha_{l}\left(\mathcal{I}_{1}, \mathcal{I}_{2}\right)$ be functions of these two invariants, so that

$$
\sum_{l=1}^{18} \alpha_{l} \mathfrak{D I}_{2, l} \stackrel{\text { l.o. }}{=} 0
$$

then

$$
\forall l \in\{1, \ldots, 18\}: \alpha_{l}=0
$$

Proof. We use the same strategy as in the proof of lemma 4.4. We multiply eq. C.3.4 by $\mathfrak{I}_{2, k}$ for all $k=1, \ldots, 18$ and get 18 equation,

$$
0 \stackrel{\text { l.o. }}{=} \mathfrak{I}_{2, k} \sum_{l=1}^{18} \alpha_{l} \mathfrak{D I}_{2, l}=\sum_{(q, r, s, t, u, v)} \alpha_{l} \underbrace{I_{\text {imn }} I_{\text {jop }}}_{\mathfrak{I}_{2, k}} \mathfrak{D} \underbrace{I_{q r s} I_{\text {tuv }}}_{\mathfrak{I}_{2, l}} .
$$

The sum on the right side is over a set of tuples, $(q, r, s, t, u, v)$, which contains the 18 tuples, which are $\mathfrak{I}_{2, l}$.

We immediately see, that a number of summands are zero. If $k \leq 4$, then $m=o$ and $n=p$ and eq. (C.3.3) only leaves terms with $(m \neq r \vee n \neq s) \wedge(m \neq u \vee n \neq v)$. The other way around is, of course, also true. If $l \leq 4$, then $r=u$ and $s=v$ and eq. C.3.3 only leaves terms with $(r \neq m \vee s \neq n) \wedge(r \neq o \vee s \neq t)$. Due to the product rule there may not be four times the same $\Theta_{1(i)}$ and $\bar{\Theta}_{1(i)}, i=1,2$, in one summand. This gives the conditions $(m+o+r+u) \bmod 4 \neq 0$ and $(n+p+s+v) \bmod 4 \neq 0$. Furthermore for every invariant, $I_{q r s}$, the four derivatives, $D_{1-}, D_{2-}, \bar{D}_{3+}$ and $\bar{D}_{4+}$, vanish or leave in lowest order a term proportional to $\Theta_{1(r)}$ or $\bar{\Theta}_{1(s)}$ (cf. eqns. (C.1.14-(C.1.19)). So terms can only be non-zero in one of these derivatives, if $m+o+r+u=6$ or $n+p+s+v=6$. This gives eighteen equations, which are valid for all four invariants. For these we apply the product rule in each summand. There are three cases,

$$
\begin{aligned}
& I_{\text {imn }} I_{\text {jop }} \mathfrak{D} I_{\text {qrs }} I_{\text {tuv }} \\
& = \begin{cases}I_{\text {imn }} I_{\text {jop }} I_{\text {qrs }} \mathfrak{D} I_{\text {tuv }} & m=o=u \vee m=p=v \\
I_{\text {imn }} I_{\text {jop }} I_{\text {tuv }} \mathfrak{D} I_{\text {qrs }} & m=o=r \vee m=p=s \\
I_{\text {imn }} I_{\text {jop }} I_{\text {qrs }} \mathfrak{D} I_{\text {tuv }}+I_{\text {imn }} I_{\text {jop }} I_{\text {tuv }} \mathfrak{D} I_{\text {qrs }} & \text { else. }\end{cases}
\end{aligned}
$$

At last we can replace each product of three invariants in front of a derivative by an invariant $\mathfrak{I}_{3, h}$ with $h \in\{1, \ldots, 8\}$, if it is not yet one of the latter. We call this set of simplified equations $E_{\text {all }}$. The remaining summands in these equations have the form $I_{1 i j}{ }^{2} I_{m n o} \mathfrak{D} I_{p q r}$ with new indices $i, j, m, n, o, p, q, r=1,2$ and $n \neq i$ and $o \neq j$ and $n=q \vee o=r$.

To get equations, which are easier to solve, we do not look at all derivatives at once, but only on one. Eq. C.3.4 is assumed to vanish for each of the four 
derivatives in this lemma. The derivative $D_{2-}$ of the invariants, $I_{i j 2}$, vanishes. $D_{2-} I_{i j 1}$ has an overall factor $\Theta_{1(j)}$ in lowest order. This leaves only the summands $I_{1 i j}{ }^{2} I_{m n o} \mathfrak{D} I_{p n 1}$ with $n \neq i$ and $o \neq j$. We denote this set of equations as $E_{2}$ refering to the derivative $D_{2-}$.

This set of equations now contains already very simple ones. For the second and the forth equation - numbered by $k$ in eq. C.3.6 - only one term is left,

$$
\begin{aligned}
& 2 \alpha_{3} I_{112}{ }^{2} I_{121} D_{2-} I_{121}=0, \\
& 2 \alpha_{1} I_{122}{ }^{2} I_{111} D_{2-} I_{111}=0 .
\end{aligned}
$$

Thus $\alpha_{1}=\alpha_{3}=0$.

At this point we need the following two identities, which are valid to lowest order and can be easily seen with the help of eq. C.1.16) and eq. A.1.9,

$$
\begin{gathered}
I_{2 i j} D_{2-} I_{2 i 1} \stackrel{\text { l.o. }}{=} \underbrace{\frac{X_{1(2) 0}^{2}}{X_{1(1) 0}^{2}} I_{1 i j} D_{2-} I_{1 i 1},}_{=: A_{0}} \\
I_{1 i j} D_{2-} I_{2 i 1}+I_{2 i j} D_{2-} I_{1 i 1} \stackrel{\text { l.o. }}{=} \underbrace{\frac{X_{1(1) 0} \cdot X_{1(2) 0}}{X_{1(1) 0}}}_{=: A_{1}} I_{1 i j} D_{2-} I_{1 i 1} .
\end{gathered}
$$

These can be used for the further simplification of $E_{2}$. This way the first equation of $E_{2}$ gets,

$$
\left(\alpha_{10} I_{111}^{2} I_{122}+\alpha_{12} A_{1} I_{111}^{2} I_{122}-\alpha_{12} I_{111}^{2} I_{222}\right) D_{2-} I_{121}=0 .
$$

If we now look at

$$
\begin{aligned}
& I_{1 i j} D_{2-} I_{1 i 1} \stackrel{\text { 1.o. }}{=} \mathrm{i} \frac{X_{1(2) 0}^{2}}{X_{1(1) 0}{ }^{4}} \tilde{\mathrm{x}}_{12}^{-1} \bar{\Theta}_{1(j)} \Theta_{1(i)} \tilde{\Theta}_{1(i)}, \\
& I_{2 i j} D_{2-} I_{1 i 1} \stackrel{\text { l.o. }}{=} \mathrm{i} \frac{1}{2 X_{1(1) 0} 4} \tilde{\mathrm{x}}_{12}^{-1} \tilde{\mathrm{X}}_{1(1)} \mathrm{X}_{1(2)} \bar{\Theta}_{1(j)} \Theta_{1(i)} \tilde{\Theta}_{1(i)}
\end{aligned}
$$

they differ by linear independent hermitian matrices. Thus the coefficient functions in eq. C.3.12 of these two terms have to be separately zero. It follows $\alpha_{10}=\alpha_{12}=$ 0 . Similar we get from the tenth equation of $E_{2}$ using $\alpha_{1}=0$,

$$
-\frac{1}{2}\left(\alpha_{5} I_{111}+\alpha_{15} I_{211}\right) I_{122}^{2} D_{2-} I_{111}=0,
$$

the next two vanishing coefficient functions, $\alpha_{5}=\alpha_{15}=0$.

If we look at this stage at the second equation of $E_{\text {all }}$ and choose the derivative $\bar{D}_{4+}$, we are left with

$$
\alpha_{6} I_{112}^{2} I_{121} \bar{D}_{4+} I_{111}+\alpha_{11} I_{112}^{2} I_{221} \bar{D}_{4+} I_{111}=0 .
$$

As in the two cases above, it follows $\alpha_{6}=\alpha_{11}=0$. 
As in the proof of lemma (4.4) we cannot separate subsets of the six coefficient functions, $\alpha_{i}$, with $i=7,8,13,14,17,18$, from the other coefficient functions. With the help of the identity,

$$
I_{1 j k}^{2} I_{p m n} D_{2-} I_{l m 1} \stackrel{\text { l.o. }}{=} I_{1 m k}^{2} I_{p j n} D_{2-} I_{l j 1}
$$

which we only need in lowest order and in this order can be shown easily with eqns. C.1.16, 4.3.21 and 4.3.22, we simplify the 7th, 13th, 14th, 17th and 18th equation of $E_{2}$ :

$$
\begin{aligned}
0 \stackrel{\text { l.o. }}{=} & \left(A_{0} A_{1} \alpha_{17}+A_{0}^{2} \alpha_{18}+\frac{1}{2} A_{0} \alpha_{7}-A_{0} A_{1} \alpha_{14}+\frac{1}{2} A_{0} A_{1} \alpha_{14}\right) I_{122}^{2} I_{111} D_{2-} I_{111} \\
& +\left(A_{1} \alpha_{8}-A_{1} \alpha_{7}-\frac{1}{2} A_{0} \alpha_{13}-\frac{1}{2} A_{0} \alpha_{14}\right) I_{122}{ }^{2} I_{211} D_{2-} I_{111}, \\
0 \stackrel{\text { l.o. }}{=} & \left(A_{0} \alpha_{17}+A_{0} A_{1} \alpha_{18}+\frac{1}{2} A_{0} \alpha_{13}+A_{0} \alpha_{14}\right) I_{122}{ }^{2} I_{111} D_{2-} I_{111} \\
& +\left(\alpha_{8}-\frac{1}{2} \alpha_{7}\right) I_{122}{ }^{2} I_{211} D_{2-} I_{111} \\
0 \stackrel{\text { l.o. }}{=} & \left(-\frac{1}{2} A_{0} \alpha_{17}-\frac{1}{2} A_{0} A_{1} \alpha_{18}+A_{0} \alpha_{14}\right) I_{122}{ }^{2} I_{111} D_{2-} I_{111} \\
& +\left(-\frac{1}{2} \alpha_{8}+\frac{1}{2} A_{0} \alpha_{18}+A_{1} \alpha_{13}+\alpha_{7}\right) I_{122}{ }^{2} I_{211} D_{2-} I_{111}, \\
0 \stackrel{\text { l.o. }}{=} & \left(-\frac{1}{2} A_{0} \alpha_{17}-\frac{1}{2} A_{0} A_{1} \alpha_{18}+A_{1} \alpha_{7}+A_{0} \alpha_{13}+A_{1}^{2} \alpha_{14}\right) I_{122}{ }^{2} I_{111} D_{2-} I_{111} \\
& +\left(\frac{1}{2} A_{0} \alpha_{18}-\frac{1}{2} \alpha_{8}+A_{1} \alpha_{14}\right) I_{122}{ }^{2} I_{211} D_{2-} I_{111}, \\
0 \stackrel{\text { l.o. }}{=} & \left(\alpha_{7}-\frac{1}{2} \alpha_{8}-\frac{1}{2} A_{1} \alpha_{17}-A_{1}^{2} \alpha_{18}+\frac{1}{2} A_{0} \alpha_{18}+A_{1} \alpha_{13}+A_{1} \alpha_{14}\right) \\
& \cdot I_{122}{ }^{2} I_{111} D_{2-} I_{111}+\left(\frac{1}{2} \alpha_{17}+A_{1} \alpha_{18}-\alpha_{14}\right) I_{122}^{2} I_{211} D_{2-} I_{111},
\end{aligned}
$$

with $A_{0}=X_{1(2) 0}{ }^{2} / X_{1(1) 0}{ }^{2}$ and $A_{1}=\left(X_{1(1) 0} \cdot X_{1(2) 0}\right) / X_{1(1) 0}{ }^{2}$. Every bracket in these five equations has to be zero. There is only the trivial solution, $\alpha_{7}=\alpha_{8}=$ $\alpha_{13}=\alpha_{14}=\alpha_{17}=\alpha_{18}=0$.

As we have shown for most coefficient functions, that they are zero, the other ones, $\alpha_{2}, \alpha_{4}, \alpha_{9}$ and $\alpha_{16}$, appear in very simple equations. The third equation of $E_{\text {all }}$ - the one, for which eq. C.3.4 is multiplied by $\mathfrak{I}_{2,3}-$ for $\bar{D}_{3+}$ simplifies to

$$
\left(\alpha_{9} I_{112}+\alpha_{16} I_{212}\right) I_{121}^{2} \bar{D}_{3+} I_{122}=0,
$$

so that $\alpha_{9}=\alpha_{16}=0$. The first and the third equation of $E_{\text {all }}$ for either $D_{1-}$ or $\bar{D}_{4+}$ now only contain $\alpha_{4}$ and $\alpha_{2}$, respectively, and it directly follows $\alpha_{2}=\alpha_{4}=0$. This completes the proof. 


\section{C.3.2 Proof of Lemma 5.4}

Lemma 5.4 (restated). Let $\alpha_{l}\left(\mathcal{I}_{1}, \mathcal{I}_{2}\right)$ be functions of these two invariants, so that

$$
\sum_{l=1}^{8} \alpha_{l} \mathfrak{D I}_{3, l} \stackrel{\text { l.o. }}{=} 0
$$

then

$$
\forall l \in\{1, \ldots, 8\}: \alpha_{l}=0
$$

Proof. We multiply eq. C.3.24 by $\mathfrak{I}_{1, p}=I_{i j k}$ and can split the summands in three groups,

$$
\begin{aligned}
0 \stackrel{\text { l.o. }}{=} \sum_{(m, n, r, s, t) \hat{=} l=1}^{8} \alpha_{l} I_{i j k} \mathfrak{D} I_{1 m n}{ }^{2} I_{r s t}= & \sum_{(m, n, r) \hat{=} l \in \mathfrak{S}_{1, p}} \alpha_{l} I_{i j k} \mathfrak{D} I_{1 m n}{ }^{2} I_{r j k} \\
& +\sum_{(n, r, s) \hat{=} l \in \mathfrak{S}_{2, p}} \alpha_{l} I_{i j k} \mathfrak{D} I_{1 j n}{ }^{2} I_{r m k} \\
& +\sum_{(m, r, t) \hat{=} l \in \mathfrak{S}_{3, p}} \alpha_{l} I_{i j k} \mathfrak{D} I_{1 m k}{ }^{2} I_{r j n},
\end{aligned}
$$

with $m \neq s$ and $n \neq t$ on the left hand side, $m \neq j$ and $n \neq k$ on the right hand side and sets $\mathfrak{S}_{1, p}, \mathfrak{S}_{2, p}, \mathfrak{S}_{3, p} \subset\{1, \ldots, 8\}$, which are disjunct for each $p$. The sums are over indices $l$ of $\mathfrak{I}_{3, l}$ in eq. (C.3.24), which correspond to tuples of indices appearing in the monomial $\mathfrak{I}_{3, l}$, when it is written in terms of $I_{r s t}$.

We look at the derivatives in the second and third sum in eq. (C.3.26):

$$
\begin{aligned}
I_{i j k} \mathfrak{D} I_{1 j n}{ }^{2} I_{r m k} & =I_{i j k} I_{1 j n} I_{r m k} \mathfrak{D} I_{1 j n} \\
& =-\frac{1}{2}\left(K_{i r} I_{1 j k}{ }^{2} I_{1 m n}+L_{i r} I_{1 j k}{ }^{2} I_{2 m n}\right) \mathfrak{D} I_{1 j n}, \\
I_{i j k} \mathfrak{D} I_{1 m k}{ }^{2} I_{r j n} & =I_{i j k} I_{1 m k} I_{r j n} \mathfrak{D} I_{1 m k} \\
& =-\frac{1}{2}\left(K_{i r}^{\prime} I_{1 j k}{ }^{2} I_{1 m n}+L_{i r}^{\prime} I_{1 j k}{ }^{2} I_{2 m n}\right) \mathfrak{D} I_{1 m k},
\end{aligned}
$$

with suitable functions $K_{i r}, L_{i r}, K_{i r}^{\prime}$ and $L_{i r}^{\prime}$ resulting from eqns. 4.3.52, 4.3.53 and 4.3.54.

For $k=2$ and for the derivative $D_{2-}$ the third sum on the right hand side of eq. (C.3.26) vanishes (cf. eq. (C.1.15). Because of (C.3.27), $n=1$ and eq. (C.1.16) the appearing derivative in the second sum is in lowest order proportional to $\Theta_{1(j)}$ and this sum also is zero. Hence

$$
0 \stackrel{\text { l.o. }}{=} \sum_{(m, n, r, s, t) \hat{=} l=1}^{8} \alpha_{l} I_{i j 2} \mathfrak{D} I_{1 m n}{ }^{2} I_{r s t} \stackrel{\text { 1.o. }}{=} \sum_{(m, r) \triangleq l \in \mathfrak{S}_{1, p}} \alpha_{l} I_{i j 2} D_{2-} I_{1 m 1}{ }^{2} I_{r j 2} .
$$


This sum has only two terms, as $m \neq j$. For the possible $i=1,2$ we get with the help of eqns. 4.3.41) and (4.3.43) the following two equations,

$$
\begin{aligned}
0 & \stackrel{\text { l.o. }}{=} 2\left(\alpha_{l_{1}}+\frac{\hat{\mathrm{X}}_{1(1)+} \cdot \hat{\mathrm{X}}_{1(2)-}}{\hat{\mathrm{X}}_{1(1)+}^{2}} \alpha_{l_{2}}\right) I_{1 j 2} I_{1 m 1} D_{2-} I_{1 m 1}, \\
0 & \stackrel{\text { l.o. }}{=} 2\left(\frac{\hat{\mathrm{X}}_{1(1)+} \cdot \hat{\mathrm{X}}_{1(2)-}}{\hat{\mathrm{X}}_{1(1)+}^{2}} \alpha_{l_{1}}+\frac{\hat{\mathrm{X}}_{1(2)-}^{2}}{\hat{\mathrm{X}}_{1(1)+}^{2}} \alpha_{l_{2}}\right) I_{1 j 2} I_{1 m 1} D_{2-} I_{1 m 1} .
\end{aligned}
$$

$\operatorname{As}\left[\left(\hat{\mathrm{X}}_{1(1)+} \cdot \hat{\mathrm{X}}_{1(2)-}\right) / \hat{\mathrm{X}}_{1(1)+}^{2}\right]^{2} \neq \hat{\mathrm{X}}_{1(2)-}^{2} / \hat{\mathrm{X}}_{1(1)+}^{2}$, we end up with $\alpha_{l_{1}}=\alpha_{l_{2}}=0$, which means in this case, $k=2, \alpha_{1}=\alpha_{2}=\alpha_{3}=\alpha_{4}=0$.

For $j=1$ and for the derivative $\bar{D}_{3+}$ the second sum vanishes (cf. eq. (C.1.17)). Because of (C.3.28), $m=2$ and eq. (C.1.18) the appearing derivative in the third sum is in lowest order proportional to $\bar{\Theta}_{1(k)}$ and this sum also is zero.

$$
0 \stackrel{\text { l.o. }}{=} \sum_{(m, n, r, s, t) \hat{=} l=1}^{8} \alpha_{l} I_{i 1 k} \mathfrak{D} I_{1 m n}{ }^{2} I_{r s t} \stackrel{\text { l.o. }}{=} \sum_{(n, r) \hat{=} l \in \mathfrak{S}_{1, p}} \alpha_{l} I_{i 1 k} D_{3+} I_{12 n}{ }^{2} I_{r 1 k} .
$$

This case has an overlap with the one, $k=2$, so that we only have one unsolved pair of equations here,

$$
\begin{aligned}
0 & \stackrel{\text { l.o. }}{=}\left(\alpha_{7}+\frac{\hat{\mathrm{X}}_{1(1)+} \cdot \hat{\mathrm{X}}_{1(2)-}}{\hat{\mathrm{X}}_{1(1)+}^{2}} \alpha_{8}\right) I_{111}{ }^{2} I_{122} \bar{D}_{3+} I_{122}, \\
0 & \stackrel{\text { l.o. }}{=}\left(\frac{\hat{\mathrm{X}}_{1(1)+} \cdot \hat{\mathrm{X}}_{1(2)-}}{\hat{\mathrm{X}}_{1(1)+}^{2}} \alpha_{7}+\frac{\hat{\mathrm{X}}_{1(2)-}^{2}}{\hat{\mathrm{X}}_{1(1)+}^{2}} \alpha_{8}\right) I_{111}{ }^{2} I_{122} \bar{D}_{3+} I_{122} .
\end{aligned}
$$

Thus we have $\alpha_{7}=\alpha_{8}=0$.

For the remaining two coefficient functions, which appear in the equations eq. C.3.26 for $j=2$ and $k=1$ with the derivative $D_{2-}$, we simplify these equations using eqns. (C.3.10) and (C.3.11),

$$
\begin{aligned}
& 0 \stackrel{\text { l.o. }}{=}\left[\left(\alpha_{5}+\alpha_{6} A_{1}\right) I_{112}^{2} I_{121}-\alpha_{5} I_{112}{ }^{2} I_{221}\right] D_{2-} I_{121} \text {, } \\
& 0 \stackrel{\text { l.o. }}{=}\left[\alpha_{6} A_{0} I_{112}{ }^{2} I_{121}+\alpha_{5} I_{112}{ }^{2} I_{221}\right] D_{2-} I_{121} \text {. }
\end{aligned}
$$

Already one of these equations together with eqns. C.3.13 and (C.3.14 results in $\alpha_{5}=\alpha_{6}=0$.

\section{C.4 R-symmetry violating partial four-point invariants}

As we saw in the end of the discussion of three point invariants, there are a few more invariants of superconformal symmetry without R-symmetry than of the whole superconformal group. 
We are left with only few possibilities for further invariants. They have to be build from the basic three point functions we defined for the construction of the full four-point invariants. As the non-nilpotent functions $\hat{\mathrm{X}}_{1(1)+}$ and $\hat{\mathrm{X}}_{1(2)-}$ have been combined in all possible ways already in section 4.2 , the new invariants have to contain the spinors. These can either be directly contracted among themselves or combined with both $\hat{\mathrm{X}}_{1(1)+}$ and $\hat{\mathrm{X}}_{1(2)-}$, which results in the following list:

$$
\begin{aligned}
G_{i j} & =\hat{\Theta}_{1(i)} \hat{\tilde{\Theta}}_{1(j)}, \\
G_{3} & =\hat{\Theta}_{1(1)} \hat{\mathrm{X}}_{1(1)+} \hat{\tilde{\mathrm{X}}}_{1(2)-} \hat{\tilde{\Theta}}_{1(2)}, \\
\bar{G}_{i j} & =\hat{\tilde{\Theta}}_{1(i)} \hat{\bar{\Theta}}_{1(j)}, \\
\bar{G}_{3} & =\hat{\tilde{\Theta}}_{1(1)} \hat{\tilde{\mathrm{X}}}_{1(1)+} \hat{\mathrm{X}}_{1(2)-} \hat{\bar{\Theta}}_{1(2)} .
\end{aligned}
$$

Some questions have to be addressed now:

1. Why are this all possibilities?

2. How do monomials of these invariants relate to each other and to the invariants of the full superconformal group?

3. And which mixed monomials of invariants of the smaller symmetry groups $(G$ 's) and the full superconformal group (I's) are there and how do they relate to each other?

Question 1: As the invariants only containing the spinors are actually already at first glance all possible combinations, this is more a question of the relations to other combinations, which contain $\hat{\mathrm{X}}_{1(1)+}$ and $\hat{\mathrm{X}}_{1(2)-}$. We discuss this only for the invariant $G_{3} . \bar{G}_{3}$ can be dealt with analoguesly.

If there are, instead of the two different spinors in $G_{3}$, twice the same spinors,

$$
\hat{\Theta}_{1(i)} \hat{\mathrm{X}}_{1(1)+} \hat{\tilde{\mathrm{X}}}_{1(2)-} \hat{\tilde{\Theta}}_{1(\mathrm{i})}
$$

one can use eq. 4.3.21 and gets a non-nilpotent invariant $\hat{X}_{1(1)+}^{\mu} \hat{X}_{1(2)-\nu}$ and $G_{i i}$.

If the two matrices between the spinors are the same, one can use the property of the Pauli matrices contracted with a symmetric tensor (cf. eq. 4.3.24) ) and gets again one of the invariants we already know and $G_{12}$.

Furthermore $\hat{\mathrm{X}}_{1(1)+}$ and $\hat{\mathrm{X}}_{1(2)-}$ can be exchange with eq. A.1.9), which gives an additional term with $\hat{X}_{1(1)+}^{\mu} \hat{X}_{1(2)-\mu}$ and $G_{12}$. By raising and lowering indices the two spinors in $G_{3}$ can be exchanged, which also exchanges $\hat{\mathrm{X}}_{1(1)+}$ and $\hat{\mathrm{X}}_{1(2)-}$ and brings us back to the last sentence.

At last one could think of the possibility to have more than two matrices between the spinors. These would be again $\hat{\mathrm{X}}_{1(1)+}$ and $\hat{\mathrm{X}}_{1(2)-}$ or functions of them. So one could resort the matrices and contract pairs of $\hat{\mathrm{X}}_{1(1)+}$ and pairs of $\hat{\mathrm{X}}_{1(2)-}$ and ends up with the $G^{\prime} s$ listed above.

Therefore there are no other possibilities for further independent invariants. 
Question 2: Due to their nilpotency not many products are possible among these invariants. There are only two independent ones, which do not lead again to the known invariants of the full superconformal group:

$$
G_{11} G_{22} \text { and } \bar{G}_{11} \bar{G}_{22} \text {. }
$$

They are each related to other products:

$$
\begin{aligned}
G_{11} G_{22}=-2 G_{12}{ }^{2} & =-2 \frac{G_{12} G_{3}}{\hat{X}_{1(1)+}^{\mu} \hat{X}_{1(2)-\nu}}=-2 \frac{G_{3}{ }^{2}}{\hat{X}_{1(1)+}^{2} \hat{X}_{1(2)-}^{2}}, \\
\bar{G}_{11} \bar{G}_{22}=-2 \bar{G}_{12}^{2} & =-2 \frac{\bar{G}_{12} \bar{G}_{3}}{\hat{X}_{1(1)+}^{\mu} \hat{X}_{1(2)-\nu}}=-2 \frac{\bar{G}_{3}^{2}}{\hat{X}_{1(1)+}^{2} \hat{X}_{1(2)-}^{2}} .
\end{aligned}
$$

We are left with products of $G$ 's and $\bar{G}$ 's. All products of $G_{i j}$ and $\bar{G}_{k l}$ are equal to invariants of lowest order $(\theta \bar{\theta})^{2}$, which were discussed in eqns. 4.3.25, 4.3.23 and 4.3.26). This has to be the case, because a product of one $G$ and one $G$ has vanishing $\mathrm{R}$-charge. So it has to be invariant under R-symmetry and thus an invariant of the full superconformal group.

A bit more complicated are the products involving $G_{3}$ or $\bar{G}_{3}$ :

$$
\begin{aligned}
G_{i i} \bar{G}_{3} & =\hat{\Theta}_{1(i)} \hat{\tilde{\Theta}}_{1(i)} \hat{\tilde{\Theta}}_{1(1)} \hat{\tilde{\mathrm{X}}}_{1(1)+} \hat{\mathrm{X}}_{1(2)-} \hat{\bar{\Theta}}_{1(2)} \\
& =-\left(\epsilon^{-1}\right)^{\alpha \beta} \hat{\Theta}_{1(i)} \hat{\tilde{\Theta}}_{1(i)} \hat{\mathrm{X}}_{1(1)+\alpha \dot{\alpha}} \hat{\bar{\Theta}}_{1(1)}^{\dot{\alpha}} \hat{\mathrm{X}}_{1(2)-\beta \dot{\beta}} \hat{\bar{\Theta}}_{1(2)}^{\dot{\beta}} \\
& =2 \hat{\Theta}_{1(i)} \hat{\mathrm{X}}_{1(1)+} \hat{\bar{\Theta}}_{1(1)} \hat{\Theta}_{1(i)} \hat{\mathrm{X}}_{1(2)-} \hat{\bar{\Theta}}_{1(2)} \\
& =2 I_{1 i 1} I_{2 i 2} .
\end{aligned}
$$

In the third line eq. (4.3.21) is used. This works analoguesly with the help of eq. (4.3.22) in order to show

$$
G_{3} \bar{G}_{i i}=2 I_{11 i} I_{22 i}
$$

For the missing products we need another small equation for the spinors, which directly follows from the property of the Pauli matrices A.1.8:

$$
\hat{\Theta}_{1(i)}^{\alpha} \hat{\bar{\Theta}}_{1(j)}^{\dot{\alpha}}=\frac{1}{2} \hat{\Theta}_{1(i)} \sigma^{\mu} \hat{\bar{\Theta}}_{1(j)} \bar{\sigma}_{\mu}^{\dot{\alpha} \alpha}
$$

Together with eq. A.1.9) we find

$$
\begin{aligned}
G_{12} \bar{G}_{3} & =\hat{\Theta}_{1(1)} \hat{\tilde{\Theta}}_{1(2)} \hat{\tilde{\Theta}}_{1(1)} \hat{\tilde{\mathrm{X}}}_{1(1)+} \hat{\mathrm{X}}_{1(2)-} \hat{\bar{\Theta}}_{1(2)} \\
& =-\frac{1}{2} \hat{\Theta}_{1(1)} \sigma^{\mu} \hat{\bar{\Theta}}_{1(2)} \hat{\tilde{\Theta}}_{1(1)} \hat{\tilde{\mathrm{X}}}_{1(1)+}\left(2 \hat{X}_{1(2)-\mu}-\sigma_{\mu} \hat{\tilde{\mathrm{X}}}_{1(2)-}\right) \hat{\tilde{\Theta}}_{1(2)} \\
& =I_{212} I_{121}+I_{111} I_{222} .
\end{aligned}
$$

In the same way one can show, that

$$
G_{3} \bar{G}_{12}=I_{112} I_{221}+I_{111} I_{222} .
$$


Question 3: To answer this question we give a list of all monomials of $G$ 's, $\bar{G}$ 's and $I_{i j k}$ sorted according to their degree in the rows and their R-charge in the columns:

\begin{tabular}{l|l|l|l|l}
2 & 1 & 0 & -1 & -2 \\
\hline \multirow{3}{*}{$G_{11} G_{22}$} & $G_{i j}, G_{3}$ & $\mathfrak{I}_{1, i}$ & $\bar{G}_{i j}, \bar{G}_{3}$ & \multirow{2}{*}{$\bar{G}_{11} \mathfrak{G}_{1, i}$} \\
& $\mathfrak{I}_{2, i}$ & $\bar{G}_{12} \mathfrak{I}_{1, i}$ \\
& $G_{12} \mathfrak{I}_{2, i}$ for $i=6,7,9,13$ & $\mathfrak{I}_{3, i}$ & $\bar{G}_{12} \mathfrak{I}_{2, i}$ for $i=5,7,10,13$ & \\
& $\mathfrak{I}_{4, i}$ & &
\end{tabular}

Once more with the help of eqns. 4.3.21 and 4.3.22 all other mixed monomials of $G$ 's and I's, which do not vanish, can be expressed by those listed above. For second degree we get

$$
\begin{aligned}
G_{11} I_{i 2 k} & =-2 G_{12} I_{i 1 k} \\
G_{22} I_{i 1 k} & =-2 G_{12} I_{i 2 k} \\
G_{3} I_{11 k} & =G_{12} \hat{X}_{1(1)+}^{2} I_{21 k} \\
G_{3} I_{22 k} & =G_{12} \hat{X}_{1(2)-}^{2} I_{12 k} \\
G_{3} I_{12 k} & =G_{12}\left(2 \hat{X}_{1(1)+}^{\mu} \hat{X}_{1(2)-\mu} I_{12 k}-\hat{X}_{1(1)+}^{2} I_{22 k}\right) \\
G_{3} I_{21 k} & =G_{12}\left(2 \hat{X}_{1(1)+}^{\mu} \hat{X}_{1(2)-\mu} I_{21 k}-\hat{X}_{1(1)+}^{2} I_{11 k}\right) \\
\bar{G}_{11} I_{i j 2} & =-2 \bar{G}_{12} I_{i j 1} \\
\bar{G}_{22} I_{i j 1} & =-2 \bar{G}_{12} I_{i j 2} \\
\bar{G}_{3} I_{1 j 1} & =\bar{G}_{12} \hat{X}_{1(1)+}^{2} I_{2 j 1} \\
\bar{G}_{3} I_{2 j 2} & =\bar{G}_{12} \hat{X}_{1(2)-}^{2} I_{1 j 2} \\
\bar{G}_{3} I_{1 j 2} & =\bar{G}_{12}\left(2 \hat{X}_{1(1)+}^{\mu} \hat{X}_{1(2)-\mu} I_{1 j 2}-\hat{X}_{1(1)+}^{2} I_{2 j 2}\right) \\
\bar{G}_{3} I_{2 j 1} & =\bar{G}_{12}\left(2 \hat{X}_{1(1)+}^{\mu} \hat{X}_{1(2)-\mu} I_{2 j 1}-\hat{X}_{1(1)+}^{2} I_{1 j 1}\right) .
\end{aligned}
$$

Due to these equations also mixed monomials of third degree can always be written in a way, which contains $G_{12}$ or $\bar{G}_{12}$ and no other $G$ 's. Furthermore we can use eqns. C.4.14 and (C.4.20 twice to relate some of the remaining monomials with each other:

$$
\begin{aligned}
G_{12} I_{i 1 k} I_{n 2 m} & =-\frac{1}{2} G_{11} I_{i 2 k} I_{n 2 m}=G_{12} I_{i 2 k} I_{n 1 m}, \\
\bar{G}_{12} I_{i 1 k} I_{n 2 m} & =-\frac{1}{2} \bar{G}_{11} I_{i 2 k} I_{n 2 m}=\bar{G}_{12} I_{i 2 k} I_{n 1 m} .
\end{aligned}
$$

If we now multiply eqns. (4.3.47), 4.3.48), 4.3.50 and 4.3.51) by $G_{12}$ and use eq. (C.4.26), we get relations for the monomials $G_{12} \Im_{2, l}$ for $l=11,16,14,18$, respec- 
tively:

$$
\begin{aligned}
& G_{12} I_{111} I_{221} \stackrel{\text { C.4.26 }}{-} \frac{1}{2}\left(G_{12} I_{111} I_{221}+G_{12} I_{211} I_{121}\right) \\
& \text { 4.3.47) } \hat{X}_{1(1)+}^{\mu} \hat{X}_{1(2)-\mu} G_{12} I_{111} I_{121} \text {, } \\
& G_{12} I_{122} I_{212} \stackrel{\text { C.4.26 }}{-} \frac{1}{2}\left(G_{12} I_{122} I_{212}+G_{12} I_{222} I_{112}\right) \\
& \stackrel{4.3 .48}{-} \hat{X}_{1(1)+}^{\mu} \hat{X}_{1(2)-\mu} G_{12} I_{112} I_{122}, \\
& G_{12} I_{122} I_{211}+G_{12} I_{111} I_{222} \stackrel{\text { C.4.26 }}{=} \frac{1}{2}\left(G_{12} I_{121} I_{212}+G_{12} I_{112} I_{221}+G_{12} I_{111} I_{222}+\right. \\
& \left.+G_{12} I_{122} I_{211}\right) \\
& \text { 4.3.50) } \hat{X}_{1(1)+}^{\mu} \hat{X}_{1(2)-\mu}\left(G_{12} I_{111} I_{122}+G_{12} I_{112} I_{121}\right) \\
& \stackrel{\text { C.4.26 }}{-} \hat{X}_{1(1)+}^{\mu} \hat{X}_{1(2)-\mu} G_{12} I_{111} I_{122} \text {, } \\
& G_{12} I_{211} I_{222} \stackrel{\text { C.4.26 }}{=} \frac{1}{2}\left(G_{12} I_{211} I_{222}+G_{12} I_{212} I_{221}\right) \\
& \stackrel{4.3 .51}{-} \hat{X}_{1(2)-}^{2}\left(G_{12} I_{111} I_{122}+G_{12} I_{112} I_{121}\right) \\
& \stackrel{\text { C.4.26 }}{=} \hat{X}_{1(2)-}^{2} G_{12} I_{111} I_{122} \text {. }
\end{aligned}
$$

Analoguesly equations can be derived for the third order monomials containing $\bar{G}_{12}$ with the help of eq. C.4.27) and the one referred to above the equal sign:

$$
\begin{aligned}
& \bar{G}_{12} I_{111} I_{212} \stackrel{4.3 .46}{=} \hat{X}_{1(1)+}^{\mu} \hat{X}_{1(2)-\mu} \bar{G}_{12} I_{111} I_{112}, \\
& \bar{G}_{12} I_{122} I_{221} \stackrel{4.3 .49}{-} \hat{X}_{1(1)+}^{\mu} \hat{X}_{1(2)-\mu} \bar{G}_{12} I_{121} I_{122}, \\
& \bar{G}_{12} I_{122} I_{211} \stackrel{4.3 .50}{-} \hat{X}_{1(1)+}^{\mu} \hat{X}_{1(2)-\mu} \bar{G}_{12} I_{111} I_{122}-\bar{G}_{12} I_{111} I_{222}, \\
& \bar{G}_{12} I_{211} I_{222} \stackrel{4.3 .51}{-} \hat{X}_{1(2)-}^{2} \bar{G}_{12} I_{111} I_{122} \text {. }
\end{aligned}
$$

This end the discussion of the monomials containing $G$ 's and the discussion of the parital four-point invariants. 


\section{Appendix D}

\section{Calculations using Maple}

\section{D.1 Procedures for the calculation of expansions in terms of monomials of $I_{i j k}$}

With all the identities from section 4.3 .2 we can compute the coefficients of the expansion of any product of two invariant functions. We define the functions $F$ and $G$ to have the expansions (cf. theorem 4.1 and eq. 4.3.119)

$$
\begin{array}{r}
F\left(\hat{X}_{1(2)-}^{2}, \hat{X}_{1(1)+} \cdot \hat{X}_{1(2)-}, I_{111}, \ldots, I_{222}\right)=\sum_{i=0}^{4} \sum_{j=1}^{n_{i}} F_{i, j}\left(\hat{X}_{1(2)-}^{2}, \hat{X}_{1(1)+} \cdot \hat{X}_{1(2)-}\right) \mathfrak{I}_{i, j} \\
G\left(\hat{X}_{1(2)-}^{2}, \hat{X}_{1(1)+} \cdot \hat{X}_{1(2)-}, I_{111}, \ldots, I_{222}\right)=\sum_{i=0}^{4} \sum_{j=1}^{n_{i}} G_{i, j}\left(\hat{X}_{1(2)-}^{2}, \hat{X}_{1(1)+} \cdot \hat{X}_{1(2)-}\right) \mathfrak{I}_{i, j} .
\end{array}
$$

Their product is the function $H$

$$
\begin{aligned}
& H\left(\hat{X}_{1(2)-}^{2}, \hat{X}_{1(1)+}^{\mu} \hat{X}_{1(2)-\mu}, I_{111}, \ldots, I_{222}\right) \\
= & (F G)\left(\hat{X}_{1(2)-}^{2}, \hat{X}_{1(1)+}^{\mu} \hat{X}_{1(2)-\mu}, I_{111}, \ldots, I_{222}\right), \\
= & \sum_{i=0}^{4} \sum_{j=1}^{n_{i}} H_{i, j}\left(\hat{X}_{1(2)-}^{2}, \hat{X}_{1(1)+}^{\mu} \hat{X}_{1(2)-\mu}\right) \Im_{i, j} .
\end{aligned}
$$

Then the coefficients $H_{i, j}$ are

$$
\begin{aligned}
H_{0,1}= & F_{0,1} G_{0,1} \\
& \forall i \in\{1, \ldots, 8\}: \\
H_{1, i}= & F_{1, i} G_{0,1}+F_{0,1} G_{1, i} \\
& \forall i \in\{1, \ldots, 4\}: \\
H_{2, i}= & F_{2, i} G_{0,1}+F_{0,1} G_{i}+F_{1, i} G_{1, i}+F_{1, i+4} G_{1, i+4} \hat{X}_{1(2)-}^{2} \\
& +F_{1, i} G_{1, i+4} \hat{X}_{1(1)+}^{\mu} \hat{X}_{1(2)-\mu}+F_{1, i+4} G_{1, i} \hat{X}_{1(1)+}^{\mu} \hat{X}_{1(2)-\mu},
\end{aligned}
$$




$$
\begin{aligned}
& H_{2,5}=F_{2,5} G_{0,1}+F_{0,1} G_{2,5}+F_{1,1} G_{1,2}+F_{1,2} G_{1,1}+F_{1,6} G_{1,5} \hat{X}_{1(2)-}^{2} \\
& +F_{1,5} G_{1,6} \hat{X}_{1(2)-}^{2}+2\left(F_{1,2} G_{1,5}+F_{1,5} G_{1,2}\right) \hat{X}_{1(1)+}^{\mu} \hat{X}_{1(2)-\mu}, \\
& H_{2,6}=F_{2,6} G_{0,1}+F_{0,1} G_{2,6}+F_{1,1} G_{1,3}+F_{1,3} G_{1,1}+F_{1,7} G_{1,5} \hat{X}_{1(2)-}^{2} \\
& +F_{1,5} G_{1,7} \hat{X}_{1(2)-}^{2}+2\left(F_{1,3} G_{1,5}+F_{1,5} G_{1,3}\right) \hat{X}_{1(1)+}^{\mu} \hat{X}_{1(2)-\mu}, \\
& H_{2,7}=F_{2,7} G_{0,1}+F_{0,1} G_{2,7}+F_{1,4} G_{1,2}+F_{1,2} G_{1,4}+F_{1,6} G_{1,8} \hat{X}_{1(2)-}^{2} \\
& +F_{1,8} G_{1,6} \hat{X}_{1(2)-}^{2}+2\left(F_{1,2} G_{1,8}+F_{1,8} G_{1,2}\right) \hat{X}_{1(1)+}^{\mu} \hat{X}_{1(2)-\mu}, \\
& H_{2,8}=F_{2,8} G_{0,1}+F_{0,1} G_{2,8}+F_{1,3} G_{1,4}+F_{1,4} G_{1,3}+F_{1,8} G_{1,7} \hat{X}_{1(2)-}^{2} \\
& +F_{1,7} G_{1,8} \hat{X}_{1(2)-}^{2}+2\left(F_{1,3} G_{1,8}+F_{1,8} G_{1,3}\right) \hat{X}_{1(1)+}^{\mu} \hat{X}_{1(2)-\mu}, \\
& H_{2,9}=F_{2,9} G_{0,1}+F_{0,1} G_{2,9}+F_{1,1} G_{1,6}+F_{1,6} G_{1,1}-F_{1,2} G_{1,5}-F_{1,5} G_{1,2} \text {, } \\
& H_{2,10}=F_{2,10} G_{0,1}+F_{0,1} G_{2,10}+F_{1,1} G_{1,7}+F_{1,7} G_{1,1}-F_{1,3} G_{1,5}-F_{1,5} G_{1,3} \text {, } \\
& H_{2,11}=F_{2,11} G_{0,1}+F_{0,1} G_{2,11}-F_{1,2} G_{1,8}-F_{1,8} G_{1,2}+F_{1,4} G_{1,6}+F_{1,6} G_{1,4} \text {, } \\
& H_{2,12}=F_{2,12} G_{0,1}+F_{0,1} G_{2,12}-F_{1,3} G_{1,8}-F_{1,8} G_{1,3}+F_{1,4} G_{1,7}+F_{1,7} G_{1,4} \text {, } \\
& H_{2,13}=F_{2,13} G_{0,1}+F_{0,1} G_{2,13}+F_{1,1} G_{1,4}+F_{1,4} G_{1,1} \\
& +2 \hat{X}_{1(1)+}^{\mu} \hat{X}_{1(2)-\mu}\left(F_{1,3} G_{1,6}+F_{1,6} G_{1,3}\right)+\hat{X}_{1(2)-}^{2}\left(F_{1,5} G_{1,8}+F_{1,8} G_{1,5}\right) \text {, } \\
& H_{2,14}=F_{2,14} G_{0,1}+F_{0,1} G_{2,14}+F_{1,2} G_{1,3}+F_{1,3} G_{1,2} \\
& +2 \hat{X}_{1(1)+}^{\mu} \hat{X}_{1(2)-\mu}\left(F_{1,3} G_{1,6}+F_{1,6} G_{1,3}\right)+\hat{X}_{1(2)-}^{2}\left(F_{1,5} G_{1,8}+F_{1,8} G_{1,5}\right) \text {, } \\
& H_{2,15}=F_{2,15} G_{0,1}+F_{0,1} G_{2,15}+F_{1,6} G_{1,7}+F_{1,7} G_{1,6}-F_{1,5} G_{1,8}-F_{1,8} G_{1,5} \text {, } \\
& H_{2,16}=F_{2,16} G_{0,1}+F_{0,1} G_{2,16}+F_{1,1} G_{1,8}+F_{1,8} G_{1,1}-F_{1,3} G_{1,6}-F_{1,6} G_{1,3} \text {, } \\
& H_{2,17}=F_{2,17} G_{0,1}+F_{0,1} G_{2,17}+F_{1,2} G_{1,7}+F_{1,7} G_{1,2}-F_{1,3} G_{1,6}-F_{1,6} G_{1,3} \text {, } \\
& H_{2,18}=F_{2,18} G_{0,1}+F_{0,1} G_{2,18}+F_{1,5} G_{1,4}+F_{1,4} G_{1,5}-F_{1,3} G_{1,6}-F_{1,6} G_{1,3} \text {, } \\
& H_{3,1}=F_{3,1} G_{0,1}+F_{0,1} G_{3,1}+F_{2,1} G_{1,4}+F_{1,4} G_{10}+F_{2,13} G_{1,1}+F_{1,1} G_{2,13} \\
& +2 \mathrm{i} \hat{X}_{1(1)+}^{\mu} \hat{X}_{1(2)-\mu}\left(F_{1,3} G_{1,6}+F_{1,6} G_{1,3}\right)+\mathrm{i} \hat{X}_{1(2)-}^{2}\left(F_{1,5} G_{1,8}+F_{1,8} G_{1,5}\right) \\
& -\frac{1}{2}\left(F_{1,1} G_{2,14}+F_{2,14} G_{1,1}+F_{1,3} G_{2,5}+F_{2,5} G_{1,3}+F_{1,2} G_{2,6}+F_{2,6} G_{1,2}\right) \\
& +\hat{X}_{1(1)+}^{\mu} \hat{X}_{1(2)-\mu}\left(F_{1,1} G_{2,18}+F_{2,18} G_{1,1}+F_{1,5} G_{2,13}+F_{2,13} G_{1,5}\right) \\
& -\hat{X}_{1(1)+}^{\mu} \hat{X}_{1(2)-\mu}\left(F_{1,5} G_{2,14}+F_{2,14} G_{1,5}\right)+\hat{X}_{1(2)-}^{2}\left(F_{1,5} G_{2,18}+F_{2,18} G_{1,5}\right) \\
& -\frac{1}{2} \hat{X}_{1(2)-}^{2}\left(F_{1,5} G_{2,17}+F_{2,17} G_{1,5}\right)+\frac{1}{2} \hat{X}_{1(2)-}^{2}\left(F_{1,1} G_{2,15}+F_{2,15} G_{1,1}\right. \\
& \left.+F_{1,7} G_{2,9}+F_{2,9} G_{1,7}+F_{1,6} G_{2,10}+F_{2,10} G_{1,6}\right), \\
& H_{3,2}=F_{3,2} G_{0,1}+F_{0,1} G_{3,2}+F_{13} G_{1,1}+F_{1,1} G_{13}+F_{2,13} G_{1,4}+F_{1,4} G_{2,13} \\
& +2 \mathrm{i} F_{1,8} G_{1,8} \hat{X}_{1(2)-}^{2}+2 \mathrm{i} F_{1,4} G_{1,8} \hat{X}_{1(1)+}^{\mu} \hat{X}_{1(2)-\mu}+2 \mathrm{i} F_{1,8} G_{1,4} \hat{X}_{1(1)+}^{\mu} \hat{X}_{1(2)-\mu} \\
& -\frac{1}{2}\left(F_{1,2} G_{2,8}+F_{2,8} G_{1,2}+F_{1,4} G_{2,14}+F_{2,14} G_{1,4}+F_{1,3} G_{2,7}+F_{2,7} G_{1,3}\right) \\
& +\hat{X}_{1(1)+}^{\mu} \hat{X}_{1(2)-\mu}\left(F_{1,4} G_{2,16}+F_{2,16} G_{1,4}+F_{1,8} G_{2,13}+F_{2,13} G_{1,8}\right) \\
& -\hat{X}_{1(1)+}^{\mu} \hat{X}_{1(2)-\mu}\left(F_{1,8} G_{2,14}+F_{2,14} G_{1,8}\right)+\hat{X}_{1(2)-}^{2}\left(F_{1,8} G_{2,16}+F_{2,16} G_{1,8}\right) \\
& -\frac{1}{2} \hat{X}_{1(2)-}^{2}\left(F_{1,8} G_{2,17}+F_{2,17} G_{1,8}\right)+\frac{1}{2} \hat{X}_{1(2)-}^{2}\left(F_{1,4} G_{2,15}+F_{2,15} G_{1,4}\right. \\
& \left.+F_{1,7} G_{2,11}+F_{2,11} G_{1,7}+F_{1,6} G_{2,12}+F_{2,12} G_{1,6}\right),
\end{aligned}
$$




$$
\begin{aligned}
& H_{3,3}=F_{3,3} G_{0,1}+F_{0,1} G_{3,3}+F_{11} G_{1,3}+F_{1,3} G_{11}+F_{2,14} G_{1,2}+F_{1,2} G_{2,14} \\
& -\frac{1}{2}\left(F_{1,1} G_{2,7}+F_{2,7} G_{1,1}+F_{1,4} G_{2,5}+F_{2,5} G_{1,4}+F_{1,2} G_{2,13}+F_{2,13} G_{1,2}\right) \\
& -\mathrm{i}\left(F_{1,6} G_{1,8}+F_{1,8} G_{1,6}\right) \hat{X}_{1(2)-}^{2}-2 \mathrm{i}\left(F_{1,2} G_{1,8}+F_{1,8} G_{1,2}\right) \hat{X}_{1(1)+}^{\mu} \hat{X}_{1(2)-\mu} \\
& +\hat{X}_{1(1)+}^{\mu} \hat{X}_{1(2)-\mu}\left(F_{1,6} G_{2,14}+F_{2,14} G_{1,6}\right)-\hat{X}_{1(1)+}^{\mu} \hat{X}_{1(2)-\mu}\left(F_{1,1} G_{2,11}\right. \\
& \left.+F_{2,11} G_{1,1}+F_{1,6} G_{2,13}+F_{2,13} G_{1,6}+F_{1,4} G_{2,9}+F_{2,9} G_{1,4}\right) \\
& -\frac{1}{2} \hat{X}_{1(2)-}^{2}\left(F_{1,5} G_{2,11}+F_{2,11} G_{1,5}+F_{1,6} G_{2,18}+F_{2,18} G_{1,6}+F_{1,6} G_{2,16}\right. \\
& \left.+F_{2,16} G_{1,6}+F_{1,8} G_{2,9}+F_{2,9} G_{1,8}\right), \\
& H_{3,4}=F_{3,4} G_{0,1}+F_{0,1} G_{3,4}+F_{12} G_{1,2}+F_{1,2} G_{12}+F_{2,14} G_{1,3}+F_{1,3} G_{2,14} \\
& -\frac{1}{2}\left(F_{1,1} G_{2,8}+F_{2,8} G_{1,1}+F_{1,4} G_{2,6}+F_{2,6} G_{1,4}+F_{1,3} G_{2,13}+F_{2,13} G_{1,3}\right) \\
& -\mathrm{i}\left(F_{1,8} G_{1,7}+F_{1,7} G_{1,8}\right) \hat{X}_{1(2)-}^{2}-2 \mathrm{i}\left(F_{1,3} G_{1,8}+F_{1,8} G_{1,3}\right) \hat{X}_{1(1)+}^{\mu} \hat{X}_{1(2)-\mu} \\
& +\hat{X}_{1(1)+}^{\mu} \hat{X}_{1(2)-\mu}\left(F_{1,3} G_{2,17}+F_{2,17} G_{1,3}+F_{1,7} G_{2,14}+F_{2,14} G_{1,7}\right) \\
& -\hat{X}_{1(1)+}^{\mu} \hat{X}_{1(2)-\mu}\left(F_{1,1} G_{2,12}+F_{2,12} G_{1,1}+F_{1,7} G_{2,13}+F_{2,13} G_{1,7}\right. \\
& \left.+F_{1,4} G_{2,10}+F_{2,10} G_{1,4}\right)+\hat{X}_{1(2)-}^{2}\left(F_{1,7} G_{2,17}+F_{2,17} G_{1,7}\right) \\
& -\frac{1}{2} \hat{X}_{1(2)-}^{2}\left(F_{1,5} G_{2,12}+F_{2,12} G_{1,5}+F_{1,7} G_{2,18}+F_{2,18} G_{1,7}+F_{1,7} G_{2,16}\right. \\
& \left.+F_{2,16} G_{1,7}+F_{1,8} G_{2,10}+F_{2,10} G_{1,8}\right) \text {, } \\
& H_{3,5}=F_{3,5} G_{0,1}+F_{0,1} G_{3,5}+F_{10} G_{1,8}+F_{1,8} G_{10}+F_{2,16} G_{1,1}+F_{1,1} G_{2,16} \\
& +\frac{1}{2}\left(F_{1,5} G_{2,14}+F_{2,14} G_{1,5}\right)-\frac{1}{2}\left(F_{1,3} G_{2,9}+F_{2,9} G_{1,3}+F_{1,6} G_{2,6}+F_{2,6} G_{1,6}\right. \\
& \left.+F_{1,1} G_{2,17}+F_{2,17} G_{1,1}+F_{1,7} G_{2,5}+F_{2,5} G_{1,7}+F_{1,2} G_{2,10}+F_{2,10} G_{1,2}\right) \\
& +\hat{X}_{1(1)+}^{\mu} \hat{X}_{1(2)-\mu}\left(F_{1,5} G_{2,16}+F_{2,16} G_{1,5}\right)-\frac{1}{2} \hat{X}_{1(2)-}^{2}\left(F_{1,5} G_{2,15}+F_{2,15} G_{1,5}\right) \\
& -\hat{X}_{1(1)+}^{\mu} \hat{X}_{1(2)-\mu}\left(F_{1,1} G_{2,15}+F_{2,15} G_{1,1}+F_{1,7} G_{2,9}+F_{2,9} G_{1,7}\right. \\
& \left.+F_{1,6} G_{2,10}+F_{2,10} G_{1,6}\right) \text {, } \\
& H_{3,6}=F_{3,6} G_{0,1}+F_{0,1} G_{3,6}+F_{11} G_{1,7}+F_{1,7} G_{11}+F_{2,17} G_{1,2}+F_{1,2} G_{2,17} \\
& +\frac{1}{2}\left(F_{1,1} G_{2,11}+F_{2,11} G_{1,1}+F_{1,6} G_{2,13}+F_{2,13} G_{1,6}+F_{1,4} G_{2,9}+F_{2,9} G_{1,4}\right) \\
& -\frac{1}{2}\left(F_{1,2} G_{2,16}+F_{2,16} G_{1,2}+F_{1,8} G_{2,5}+F_{2,5} G_{1,8}+F_{1,2} G_{2,18}+F_{2,18} G_{1,2}\right. \\
& \left.+F_{1,5} G_{2,7}+F_{2,7} G_{1,5}\right)+\hat{X}_{1(2)-}^{2}\left(F_{1,6} G_{2,15}+F_{2,15} G_{1,6}\right) \\
& +\hat{X}_{1(1)+}^{\mu} \hat{X}_{1(2)-\mu}\left(F_{1,2} G_{2,15}+F_{2,15} G_{1,2}+F_{1,6} G_{2,17}+F_{2,17} G_{1,6}\right), \\
& H_{3,7}=F_{3,7} G_{0,1}+F_{0,1} G_{3,7}+F_{12} G_{1,6}+F_{1,6} G_{12} \\
& +\frac{1}{2}\left(F_{1,1} G_{2,12}+F_{2,12} G_{1,1}+F_{1,7} G_{2,13}+F_{2,13} G_{1,7}+F_{1,4} G_{2,10}+F_{2,10} G_{1,4}\right) \\
& -\frac{1}{2}\left(F_{1,3} G_{2,16}+F_{2,16} G_{1,3}+F_{1,8} G_{2,6}+F_{2,6} G_{1,8}+F_{1,3} G_{2,18}+F_{2,18} G_{1,3}\right. \\
& \left.+F_{1,5} G_{2,8}+F_{2,8} G_{1,5}\right)+\hat{X}_{1(1)+}^{\mu} \hat{X}_{1(2)-\mu}\left(F_{1,3} G_{2,15}+F_{2,15} G_{1,3}\right) \\
& +\hat{X}_{1(2)-}^{2}\left(F_{1,7} G_{2,15}+F_{2,15} G_{1,7}\right) \text {, }
\end{aligned}
$$




$$
\begin{aligned}
& H_{3,8}=F_{3,8} G_{0,1}+F_{0,1} G_{3,8}+F_{13} G_{1,5}+F_{1,5} G_{13}+F_{2,18} G_{1,4}+F_{1,4} G_{2,18} \\
& +\frac{1}{2}\left(F_{1,8} G_{2,14}+F_{2,14} G_{1,8}\right)+\hat{X}_{1(1)+}^{\mu} \hat{X}_{1(2)-\mu}\left(F_{1,8} G_{2,18}+F_{2,18} G_{1,8}\right) \\
& -\frac{1}{2}\left(F_{1,2} G_{2,12}+F_{2,12} G_{1,2}+F_{1,7} G_{2,7}+F_{2,7} G_{1,7}+F_{1,4} G_{2,17}+F_{2,17} G_{1,4}\right. \\
& \left.+F_{1,3} G_{2,11}+F_{2,11} G_{1,3}+F_{1,6} G_{2,8}+F_{2,8} G_{1,6}\right) \\
& -\hat{X}_{1(1)+}^{\mu} \hat{X}_{1(2)-\mu}\left(F_{1,4} G_{2,15}+F_{2,15} G_{1,4}+F_{1,7} G_{2,11}+F_{2,11} G_{1,7}+F_{1,6} G_{2,12}\right. \\
& \left.+F_{2,12} G_{1,6}\right)-\frac{1}{2} \hat{X}_{1(2)-}^{2}\left(F_{1,8} G_{2,15}+F_{2,15} G_{1,8}\right), \\
& H_{4,1}=F_{4,1} G_{0,1}+F_{0,1} G_{4,1}+F_{3,2} G_{1,1}+F_{1,1} G_{3,2}+F_{3,1} G_{1,4}+F_{1,4} G_{3,1} \\
& +F_{13} G_{10}+F_{10} G_{13}+F_{2,13} G_{2,13}+F_{3,4} G_{1,2}+F_{1,2} G_{3,4}+F_{3,3} G_{1,3} \\
& +F_{1,3} G_{3,3}+F_{11} G_{12}+F_{12} G_{11}+F_{2,14} G_{2,14}-10 F_{1,8} G_{1,8} \hat{X}_{1(2)-}^{2} \\
& -10 F_{1,4} G_{1,8} \hat{X}_{1(1)+}^{\mu} \hat{X}_{1(2)-\mu}-10 F_{1,8} G_{1,4} \hat{X}_{1(1)+}^{\mu} \hat{X}_{1(2)-\mu} \\
& +2 \mathrm{i} \hat{X}_{1(1)+}^{\mu} \hat{X}_{1(2)-\mu}\left(F_{1,4} G_{2,16}+F_{2,16} G_{1,4}+F_{1,8} G_{2,13}+F_{2,13} G_{1,8}\right) \\
& -2 \mathrm{i} \hat{X}_{1(1)+}^{\mu} \hat{X}_{1(2)-\mu}\left(F_{1,8} G_{2,14}+F_{2,14} G_{1,8}\right)+2 \mathrm{i} \hat{X}_{1(2)-}^{2}\left(F_{1,8} G_{2,16}+F_{2,16} G_{1,8}\right) \\
& -\mathrm{i} \hat{X}_{1(2)-}^{2}\left(F_{1,8} G_{2,17}+F_{2,17} G_{1,8}\right)+\mathrm{i} \hat{X}_{1(2)-}^{2}\left(F_{1,4} G_{2,15}+F_{2,15} G_{1,4}+F_{1,7} G_{2,11}\right. \\
& \left.+F_{2,11} G_{1,7}+F_{1,6} G_{2,12}+F_{2,12} G_{1,6}\right)-\frac{1}{2}\left(F_{2,6} G_{2,7}+F_{2,7} G_{2,6}+F_{2,5} G_{2,8}\right. \\
& \left.+F_{2,8} G_{2,5}+F_{2,13} G_{2,14}+F_{2,14} G_{2,13}\right) \\
& +\hat{X}_{1(1)+}^{\mu} \hat{X}_{1(2)-\mu}\left(F_{1,4} G_{3,5}+F_{3,5} G_{1,4}+F_{1,8} G_{3,1}+F_{3,1} G_{1,8}+F_{2,13} G_{2,16}\right. \\
& +F_{2,16} G_{2,13}+F_{1,1} G_{3,8}+F_{3,8} G_{1,1}+F_{1,5} G_{3,2}+F_{3,2} G_{1,5}+F_{2,13} G_{2,18} \\
& +F_{2,18} G_{2,13}+F_{1,3} G_{3,6}+F_{3,6} G_{1,3}+F_{1,7} G_{3,3}+F_{3,3} G_{1,7}+F_{2,14} G_{2,17} \\
& \left.+F_{2,17} G_{2,14}+F_{1,2} G_{3,7}+F_{3,7} G_{1,2}+F_{1,6} G_{3,4}+F_{3,4} G_{1,6}\right) \\
& -2 \mathrm{i} \hat{X}_{1(1)+}^{\mu} \hat{X}_{1(2)-\mu}^{2}\left(F_{1,4} G_{2,15}+F_{2,15} G_{1,4}+F_{1,7} G_{2,11}+F_{2,11} G_{1,7}+F_{1,6} G_{2,12}\right. \\
& \left.+F_{2,12} G_{1,6}\right)+2 \mathrm{i} \hat{X}_{1(1)+}^{\mu} \hat{X}_{1(2)-\mu}^{2}\left(F_{1,8} G_{2,18}+F_{2,18} G_{1,8}\right) \\
& -\mathrm{i} \hat{X}_{1(1)+}^{\mu} \hat{X}_{1(2)-\mu} \hat{X}_{1(2)-}^{2}\left(F_{1,8} G_{2,15}+F_{2,15} G_{1,8}\right) \\
& -\frac{1}{2} \hat{X}_{1(1)+}^{\mu} \hat{X}_{1(2)-\mu}\left(F_{2,14} G_{2,16}+F_{2,16} G_{2,14}+F_{2,13} G_{2,17}+F_{2,17} G_{2,13}\right. \\
& +F_{2,7} G_{2,10}+F_{2,10} G_{2,7}+F_{2,5} G_{2,12}+F_{2,12} G_{2,5}+F_{2,6} G_{2,11}+F_{2,11} G_{2,6} \\
& \left.+F_{2,8} G_{2,9}+F_{2,9} G_{2,8}+F_{2,18} G_{2,14}+F_{2,14} G_{2,18}\right) \\
& +\hat{X}_{1(2)-}^{2}\left(F_{1,5} G_{3,8}+F_{3,8} G_{1,5}+F_{1,7} G_{3,6}+F_{3,6} G_{1,7}+F_{1,6} G_{3,7}+F_{3,7} G_{1,6}\right. \\
& \left.+F_{1,8} G_{3,5}+F_{3,5} G_{1,8}+F_{2,16} G_{2,16}+F_{2,17} G_{2,17}+F_{2,18} G_{2,18}\right) \\
& +\left(\hat{X}_{1(1)+}^{\mu} \hat{X}_{1(2)-\mu}\right)^{2}\left(F_{2,16} G_{2,18}+F_{2,18} G_{2,16}+F_{2,14} G_{2,15}+F_{2,15} G_{2,14}\right) \\
& -\left(\hat{X}_{1(1)+}^{\mu} \hat{X}_{1(2)-\mu}\right)^{2}\left(F_{2,13} G_{2,15}+F_{2,15} G_{2,13}+F_{2,9} G_{2,12}+F_{2,12} G_{2,9}\right. \\
& \left.+F_{2,10} G_{2,11}+F_{2,11} G_{2,10}\right)+\frac{1}{2} \hat{X}_{1(2)-}^{2}\left(F_{2,13} G_{2,15}+F_{2,15} G_{2,13}+F_{2,9} G_{2,12}\right. \\
& \left.+F_{2,12} G_{2,9}+F_{2,10} G_{2,11}+F_{2,11} G_{2,10}\right) \\
& -\frac{1}{2} \hat{X}_{1(2)-}^{2}\left(F_{2,16} G_{2,17}+F_{2,17} G_{2,16}+F_{2,18} G_{2,17}+F_{2,17} G_{2,18}\right) \\
& -\frac{1}{2} \hat{X}_{1(1)+}^{\mu} \hat{X}_{1(2)-\mu} \hat{X}_{1(2)-}^{2}\left(F_{2,15} G_{2,16}+F_{2,16} G_{2,15}+F_{2,15} G_{2,18}+F_{2,18} G_{2,15}\right) \\
& +\hat{X}_{1(1)+}^{\mu} \hat{X}_{1(2)-\mu} \hat{X}_{1(2)-}^{2}\left(F_{2,15} G_{2,17}+F_{2,17} G_{2,15}\right)+\left(\hat{X}_{1(2)-}^{2}\right)^{2}\left(F_{2,15} G_{2,15}\right) \text {. }
\end{aligned}
$$


We programmed a computer algebra procedure, ProdExpaInv(a,b), with these equations, which enabled us to quickly calculate these products. With a small procedure, PowerExpaInv $(F, \mathrm{n})$, repeating this product $n-1$ times, the power of an invariant can be calculated.

Also a linear combination procedure, LinComExpaInv $(\mathrm{k} 1, \mathrm{~F} 1, \mathrm{k} 2, \mathrm{~F} 2)$, taking a linear combination $k_{1} F_{1}+k_{2} F_{2}$ by calculating it for every component of the 36 -tuple has often been helpful.

\section{D.2 Replacing $\hat{X}_{1(2)-}^{2}$ by other non-nilpotent invariants}

The input variables are

- the expansion, a:=array $(1 \ldots 36, \ldots)$, of the invariant, which shall replace $\hat{X}_{1(2)-}^{2}$,

- a new variable name, va, for the same invariant, which shall appear in the resulting expansion of $\hat{X}_{1(2)-}^{2}$, and

- the expansion, nxterm, which shall be inserted for $\mathfrak{X}_{3}$ (cf. eq. 4.3.117), depending only on $\mathcal{I}_{2}$ named I2v therein.

We used the latter to replace $\mathfrak{X}_{3}$ by $\mathcal{I}_{2}$ with the help of eq. 4.3.127).

In the whole procedure we replace all appearances of $\hat{X}_{1(1)+} \cdot X_{1(2)-}$ by $\hat{X}_{1(2)-}^{2}$ $(=: \mathrm{X} 2 \mathrm{mX} 2 \mathrm{~m})$ and the variable $\mathrm{Xterm}$ representing $\mathfrak{X}_{3}$. After the variable declaration we check, that $\mathrm{X} 2 \mathrm{mX} 2 \mathrm{~m}$ only appear with degree 1 .

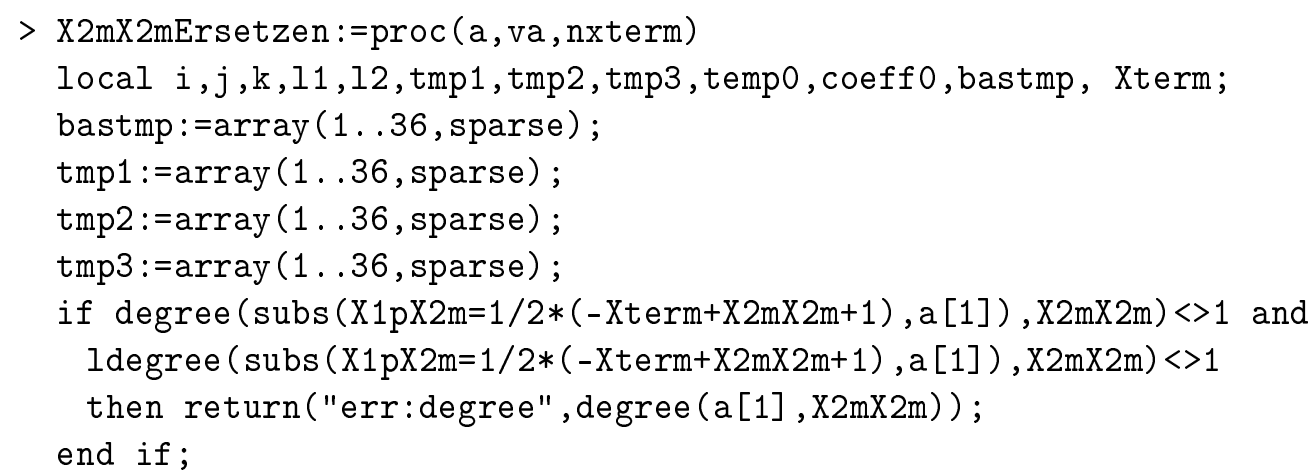

The coefficient of $\hat{X}_{1(2)-}^{2}$ is determined. The first component of the temporary result tmp1 - this component is already final - is just the new variable name divided by this coefficient. The negative values of other the components are divided by the coefficient are copied. Thus we have brought these components to the other side of the equation, which is given by the expansion a, and isolated X2mX2m. tmp2 is used as a buffer for intermediate results to avoid circle assignmets and differs only from tmp1 by the vanishing element tmp2[1].

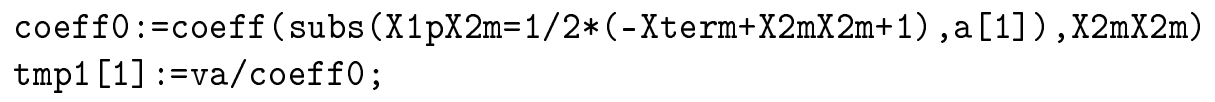




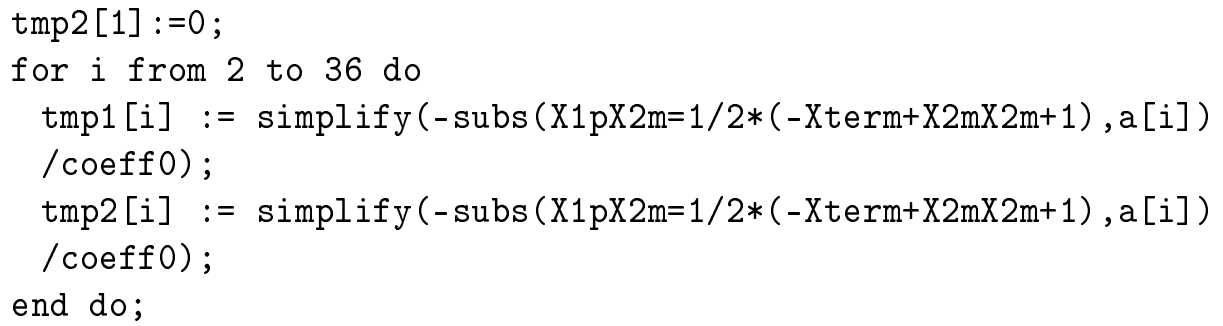

For the range of degrees of Xterm in the lowest components tmp1[1] we compute the linear combination of the expansion tmp2 and the powers of nxterm times the corresponding coefficients. This way the variable Xterm is completely replaced in the lowest component.

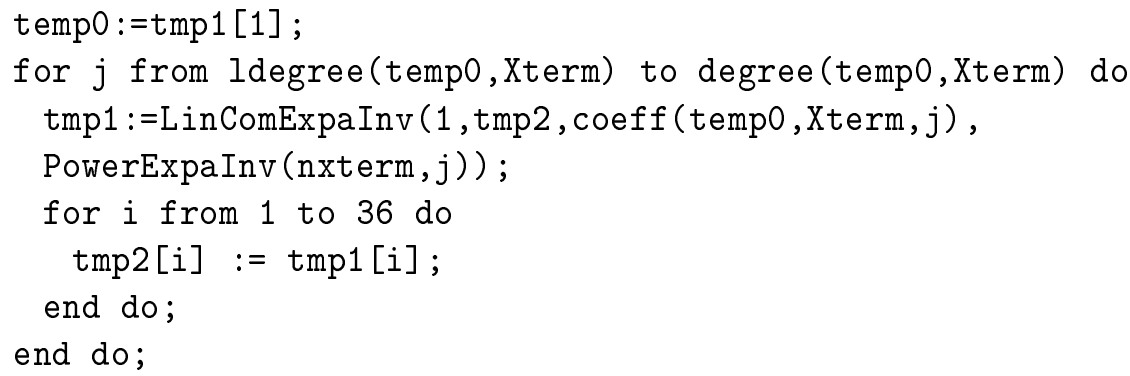

Now the loop over the other components begins. We need a copy, tmp3, of the intermediate result, tmp1, which is not changed within the loops, that follow further down.

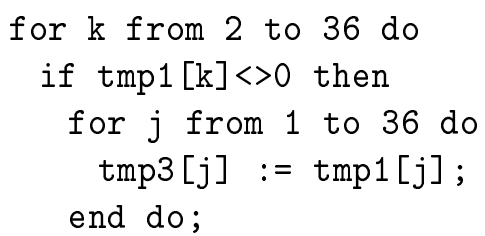

The procedure is programmed only for the case, that there are no components with negative powers of $\mathrm{X} 2 \mathrm{mX} 2 \mathrm{~m}$. The component, tmp2[k] has to be set to zero, so that this component with the needed replacement can be calculated together with the changes of the higher components and added to the expansion tmp2. This is essentially done as for the lowest component, but for both variables X2mX2m and Xterm. The procedure ProdExpaInv is used to calculate the monomials of these variables and the multiplication with a basis element bastmp, which is zero for all components but $\mathrm{k}$.

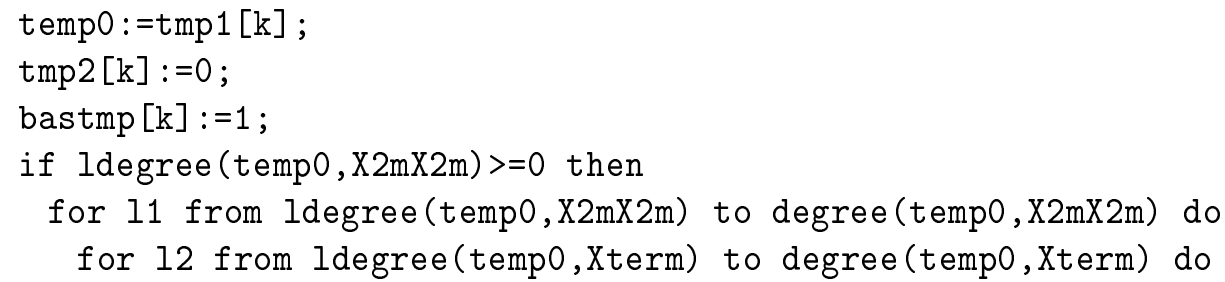




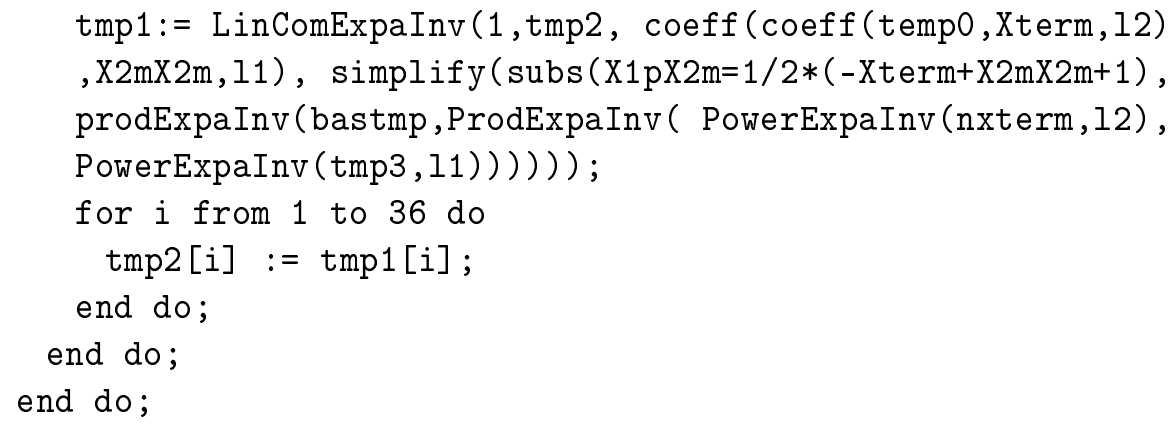

The variable for the basis element bastmp has to be set to zero again, so that it can be used for the next $\mathrm{k}$ again.

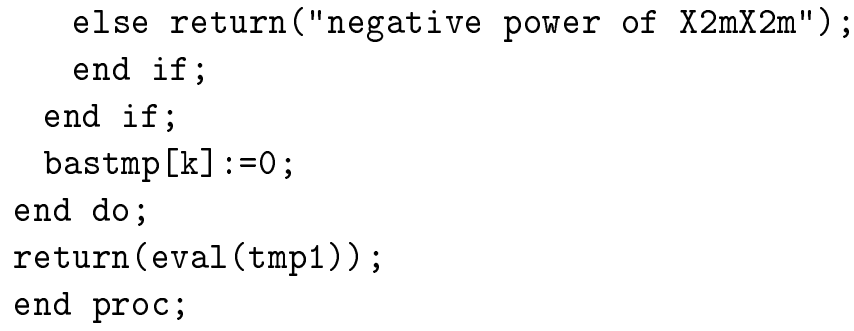

If we use the expansion of the inverse of $\mathcal{I}_{6}$ as input a and take $1 /$ I $6 \mathrm{v}$ for va, we get the expansion of $\hat{X}_{1(2)-}^{2}$ in terms of the variables I6v and I2v. If instead $\mathrm{a}$ is the expansion of $\mathcal{I}_{1}$ and $\mathrm{va}$ is $I 1 \mathrm{v}$, the elements of the resulting array are expressed in terms of $\mathrm{I} 1 \mathrm{v}$ and $\mathrm{I} 2 \mathrm{v}$.

\section{D.3 Change of coefficient functions from dependence on $\hat{X}_{1(2)-}^{2}$ and $\hat{X}_{1(1)+} \cdot \hat{X}_{1(2)-}$ to dependence on $\mathcal{I}_{2}$ and $\mathcal{I}_{1}$ or $\mathcal{I}_{6}$}

The result from the procedure in the last section and the expansion of $\mathfrak{X}_{3}$ in terms of $\mathcal{I}_{2}$ can now be used for the replacement of $\hat{X}_{1(2)-}^{2}$ and $\hat{X}_{1(1)+} \cdot \hat{X}_{1(2)-}$ in any expansion of an invariant. We present the procedure here for the replacement with $\mathcal{I}_{6}$ and $\mathcal{I}_{2}$. But, if the expansion of $\hat{X}_{1(2)-}^{2}$ in terms of $\mathcal{I}_{1}$ and $\mathcal{I}_{2}$ in eq. 4.3.129 is used, these two superconformal cross ratios are the replacements of $\hat{X}_{1(2)-}^{2}$ and $\hat{X}_{1(1)+} \cdot \hat{X}_{1(2)-}$. The argument a is an expansion of an invariant in form of an array with 36 elements depending on the variables X2mX2m and X1pX2m standing for $\hat{X}_{1(2)-}^{2}$ and $\hat{X}_{1(1)+} \cdot \hat{X}_{1(2)-}$.

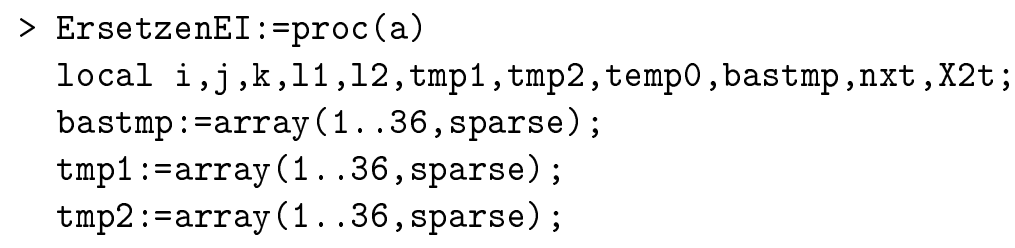


The variables nxt and X2t contain the expansions of $\mathfrak{X}_{3}$ and $\hat{X}_{1(2)-}^{2}$.

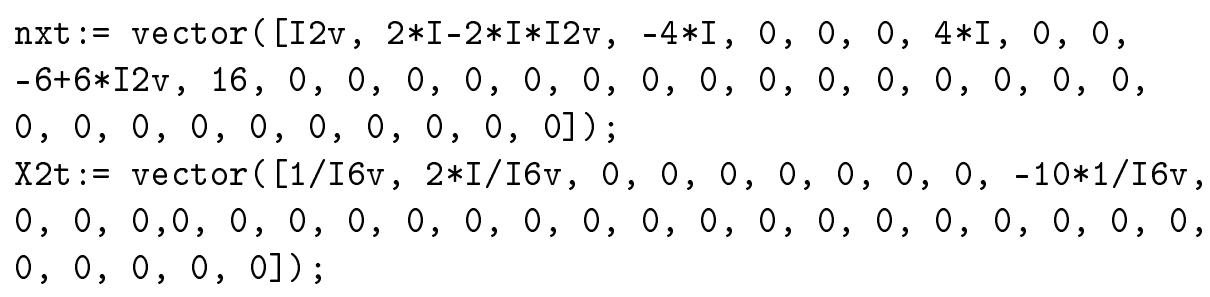

So we need to substitute $\hat{X}_{1(1)+} \cdot \hat{X}_{1(2)-}$.

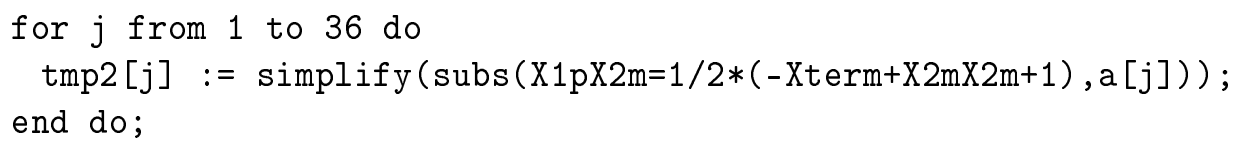

The rest of the procedure works in the same way as the one in section D.2

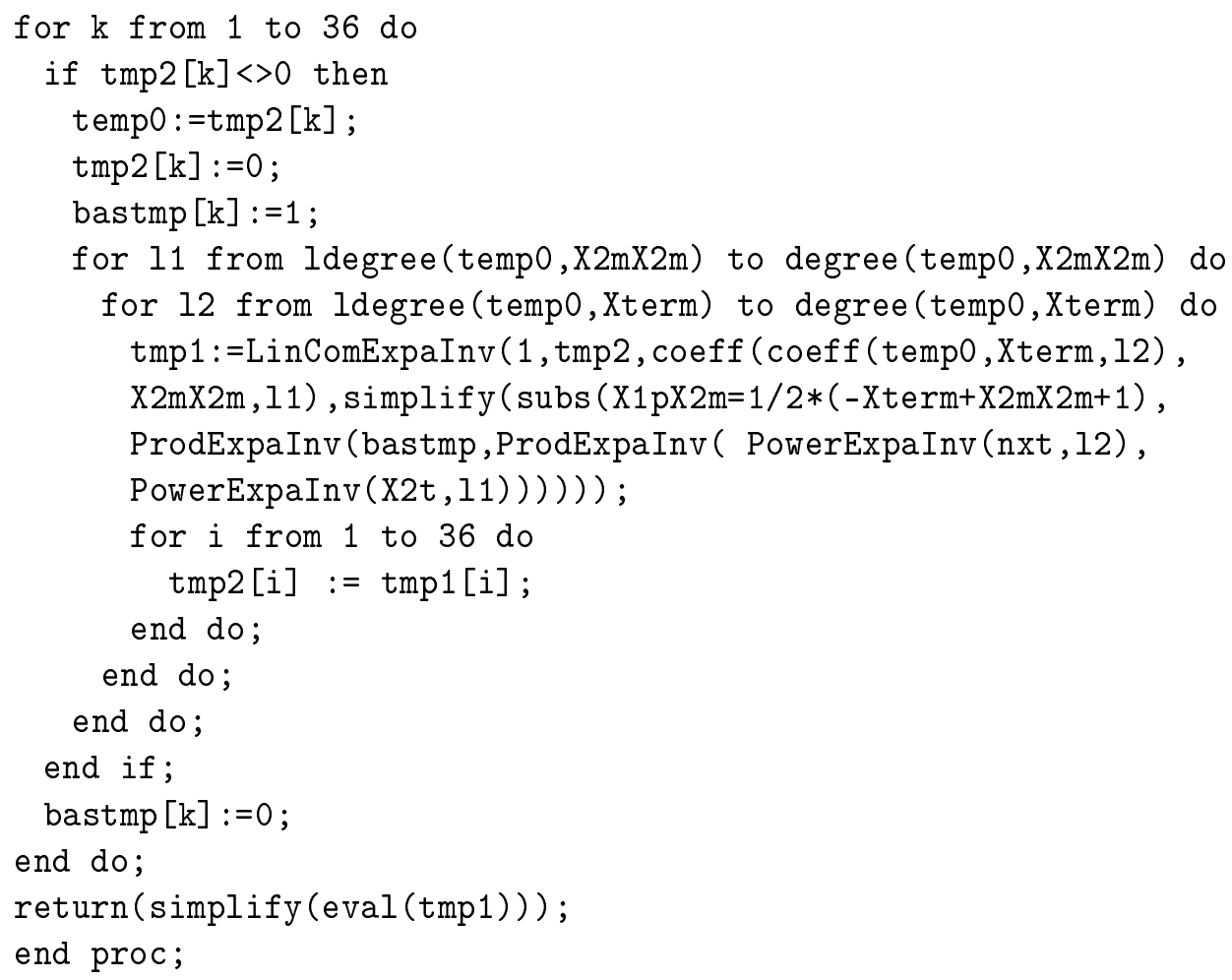

With this procedure we get the expansions with coefficient functions depending on superconformal cross ratios and want to do calculation with them. The linear combination procedure, LinComExpaInv, can still be used for them. But the usage of the product procedure, ProdExpaInv, would lead to term with X2mX2m and X1pX2m again. To avoid this one can generate most of the source code of a new product procedure, ProdEI, if we apply the substitution procedure, ErsetzenEI, to the result of the old product, ProdExpaInv $(a, b)$, with arrays a:=array $(1 \ldots 36)$ and $\mathrm{b}:=\operatorname{array}(1.36)$ with elements, which are not further declared: 
$>$ erg:=ErsetzenEI(ProdExpaInv $(\mathrm{a}, \mathrm{b}))$ :

$>\operatorname{print}\left(\operatorname{seq}\left(\left[^{\prime} \operatorname{erg}\left[{ }^{6}, i,{ }^{6}\right]:=^{6}, \operatorname{erg}[i],{ }^{\prime} ;{ }^{\prime}\right], i=10 \ldots 36\right)\right)$;

\section{D.4 Expansions of inverses and square roots of invari- ants}

With a procedure for the product of two expanded invariants - e.g. ProdExpaInv or ProdEI - the calculation of the inverse can also be easily implemented, as the inverse, for example, of function $F$ (cf. eq. (4.3.119) ) is given by

$$
\begin{aligned}
& \left(\frac{1}{F}\right)\left(\hat{X}_{1(2)-}^{2}, \hat{X}_{1(1)+}^{\mu} \hat{X}_{1(2)-\mu}, I_{111}, \ldots, I_{222}\right) \\
= & \sum_{h=0}^{4} \frac{1}{F_{0,0}{ }^{(1+h)}}\left(-\sum_{i=1}^{4} \sum_{j=1}^{n_{i}} F_{i, j} \Im_{i, j}\right)^{h} .
\end{aligned}
$$

We have generated the square route procedure from the above mentioned product procedure, ProdExpaInv (a,b), with the following few lines of source code

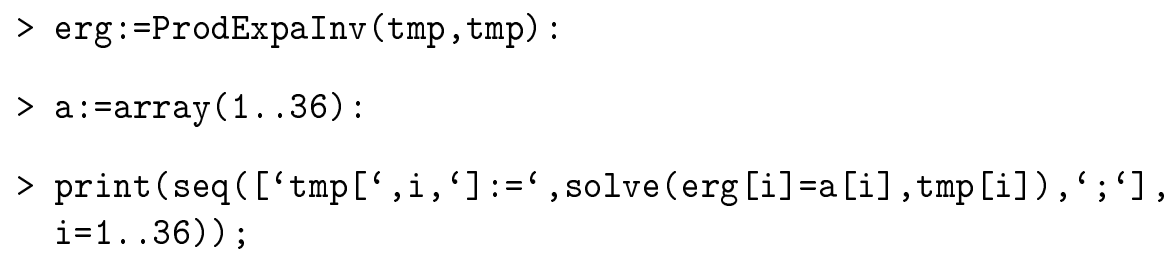

The equations can be solved one by one in the sequence, because in each solution $\operatorname{tmp}[i]$ there are only other $\operatorname{tmp}[j]$, which belong to lower order invariants. Thus, if one takes the printed output of this sequence into the source code of the procedure, every assignment of a variable tmp [i] only contains variables calculated before.

\section{D.5 Combinatorics of non-nilpotent invariants}

In this section we generate all invariants, which are ratios of squares of superconformal intervals, $x_{i j}{ }^{2}$. We describe the combinatorial approach used for this task in the paragraph about more invariant ratios surrounding eqns. 4.2.29 4.2.35.

In these calculation we treat all trees, of which we have discussed an example in that paragraph, simultaneously. We need a whole bunch of variables to keep track of our progress. We symbolize a squared interval, $x_{\bar{i}}{ }^{2}$, as a list, $[i, j]$. We assign different variables for numerators and denominators, so that these variables always appear in pairs. zaehler and nenner stand for the ratio currently treated with their numerator and denominator, respectively. zaehlerliste and nennerliste are the major working list for all generated elements of the tree, also the intermediate ones. So zaehlerliste [i] and nennerliste [i] are numerator and denominator of one ratio. counter counts in its first element the number of elements of one of these two 
equally long lists. In the second element the number of elements of current length, which is the number of intervals, $[i, j]$, in a numerator or denominator, is counted.

Now the stocks of 24 trees are generated. Of cause, one could take less, if one arguments, that there are inverses of each other among these 24 , but then one would have to argue also, which selection is taken, so that one does not miss invariants. Taking all 24 guarantees completeness and the extra calculations are not noticeable.

We get all ratios $x_{\bar{i} j}{ }^{2} / x_{\bar{i} k}{ }^{2}$ and count them in counter [2]

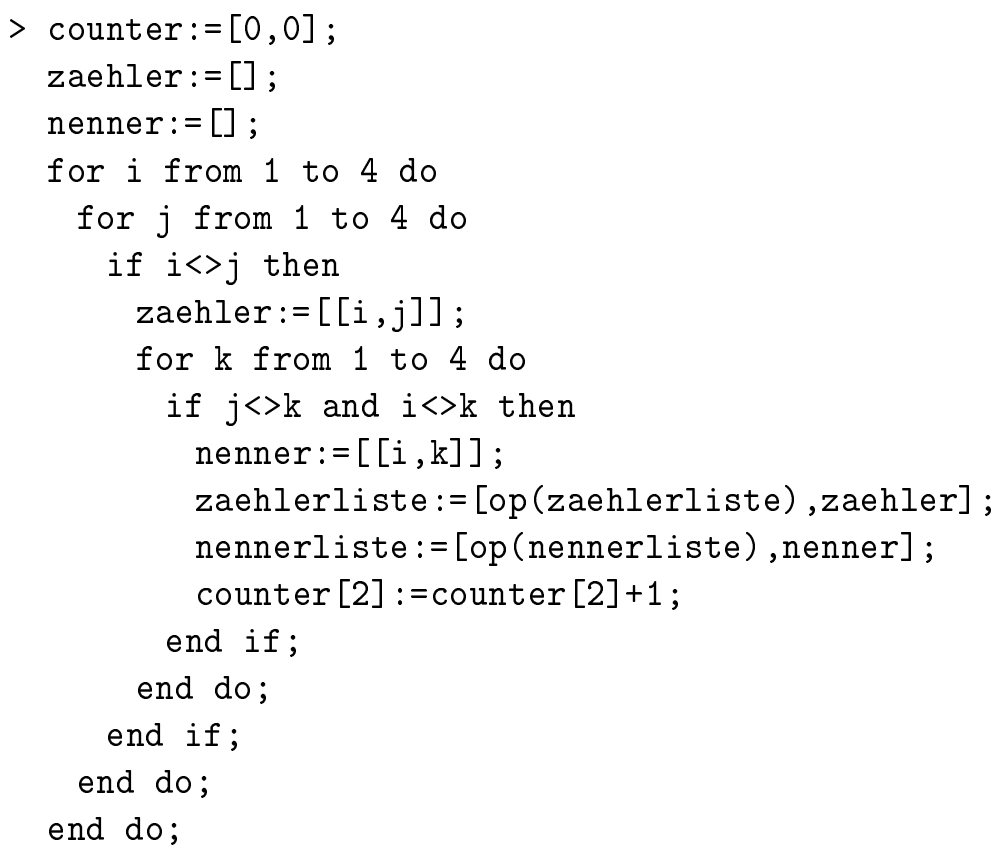

Because in each round of the contained while-loop the numerators and the denominators of the ratios get longer and longer, this current length is counted by anzahlzaehler. The lists, endzaehler and endnenner, are filled with lists of numerators resp. denominators of completed invariants in groups with the same length starting with length 1 . So endzaehler $[1, p, q]$ is the squared interval, $[i, j]$, which is the q'th interval in the numerator of the p'th finished invariant with 1 squared intervals on each side of the fraction line. The number of finished invariants with the same length $i$ is counted as an element of a list, endzaehlerzahl [i]. As we still need counter [2], we use a temporary counter, countertmp, to count the number of new ratios of length anzahlzaehler+1 generated in the current round.

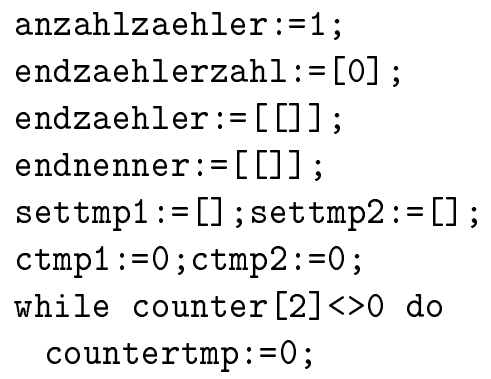


The for-loop goes through all ratios generated in the last round with the length anzahlzaehler. For each ratio the numerator and the denominator are taken out of zaehlerliste and nennerliste. Also one needs the last added interval, $[i, j]$, in the denominator, which is assigned to letzternenner.

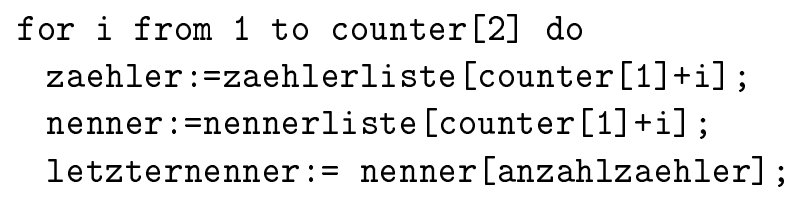

After the first round, in which there cannot appear an invariant, we test here, if for any of the found invariants its numerator and denominator can be simultaneously found in the current numerator and denominator, zaehler and nenner. This requires the simultaneous appearance of all intervals of the invariant in the current ratio. If this is not the case, testb2 is set to zero. And if it is the case for any of the invariants found so far, testb is set to one. This is one of the terminating conditions, which are described in section 4.2 .2 and prevents infinite loops and double invariants in the resulting list.

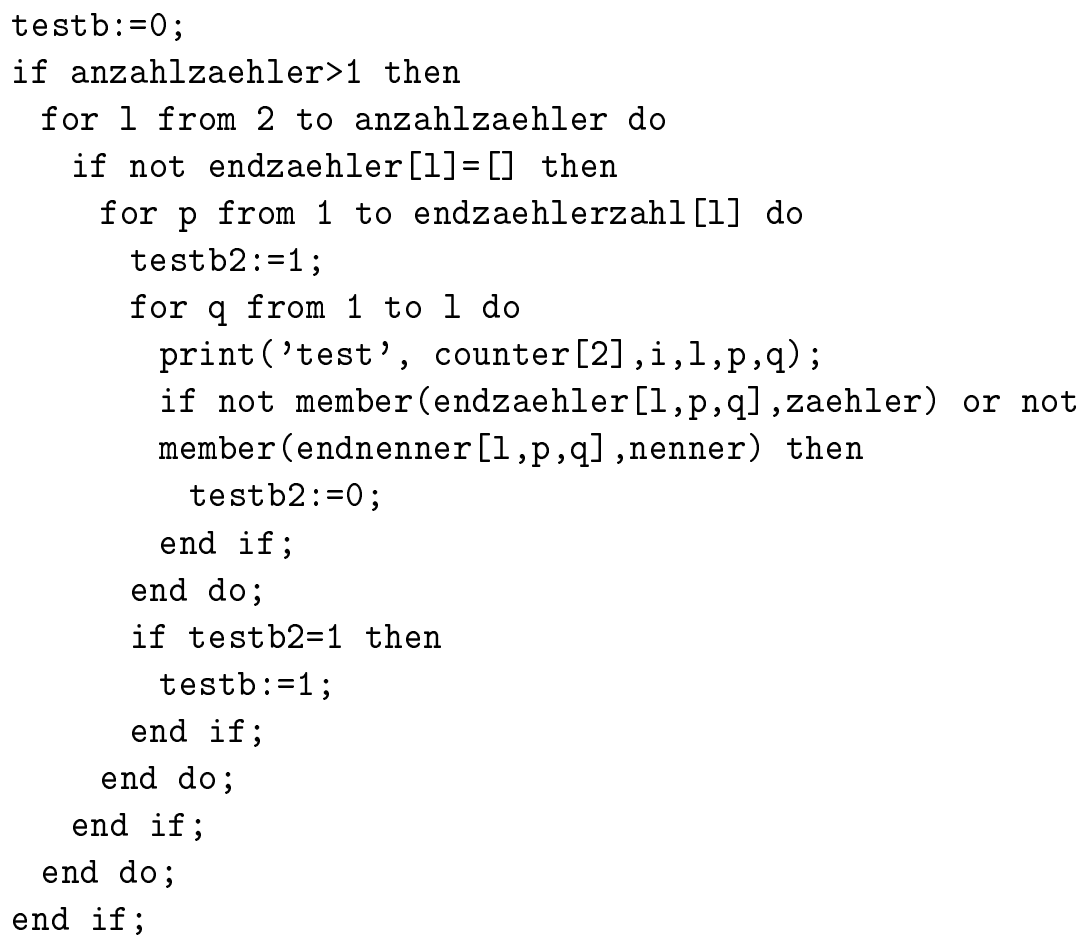

For the branches, which are not terminated, the first question to ask is, if a new invariant has been finished with zaehler and nenner, which is the other terminating condition (letzternenner [2]=zaehler $[1,2]$; this case is treated in the else below).

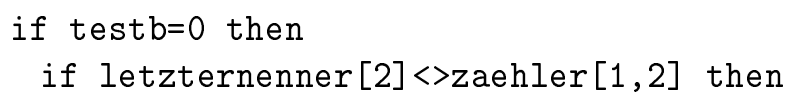


Now one new interval for the numerator and one for the denominator is searched. The new element for the numerator has be [j,letzternenner [2]] for some $j$ unequal to letzternenner [2], so that it does not appear in the denominator, yet. Otherwise it would cancel down. Once found, it is assigned to letzterzaehler. The new denominator, [letzterzaehler $[1], \mathrm{k}$ ], for some $\mathrm{k}$ unequal to the number letzterzaehler[1], has to fulfill the corresponding condition. If both could be found, it is saved as new ratio in the lists zaehlerliste and nennerliste and the counter is incremented.

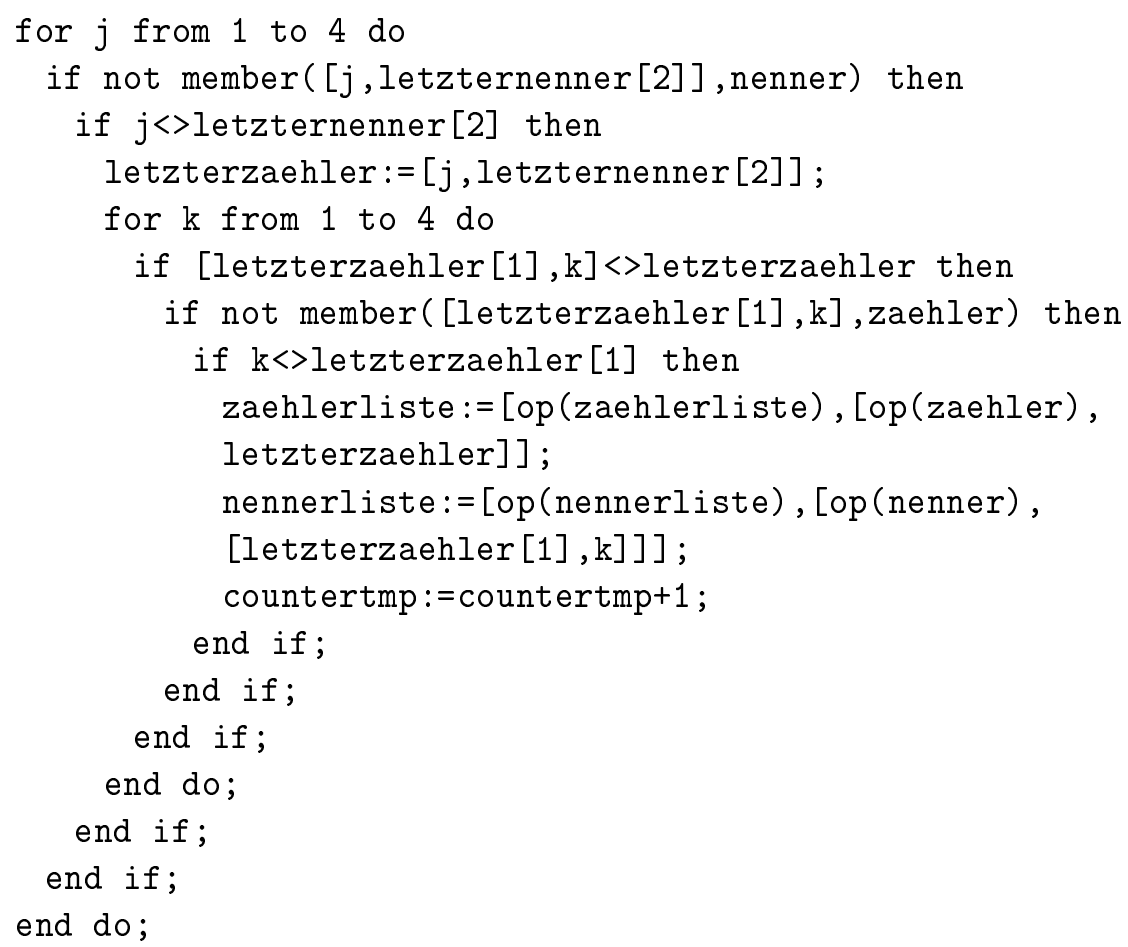

This else belongs to the query, if we have not found a new invariant. Thus for the answer " $n o$ " - so in the case of a new invariant - we have to save this invariant in the lists endzaehler [anzahlzaehler] and endnenner [anzahlzaehler], which contain those of the currently treated length anzahlzaehler, and increment the corresponding counter endzaehlerzahl [anzahlzaehler].

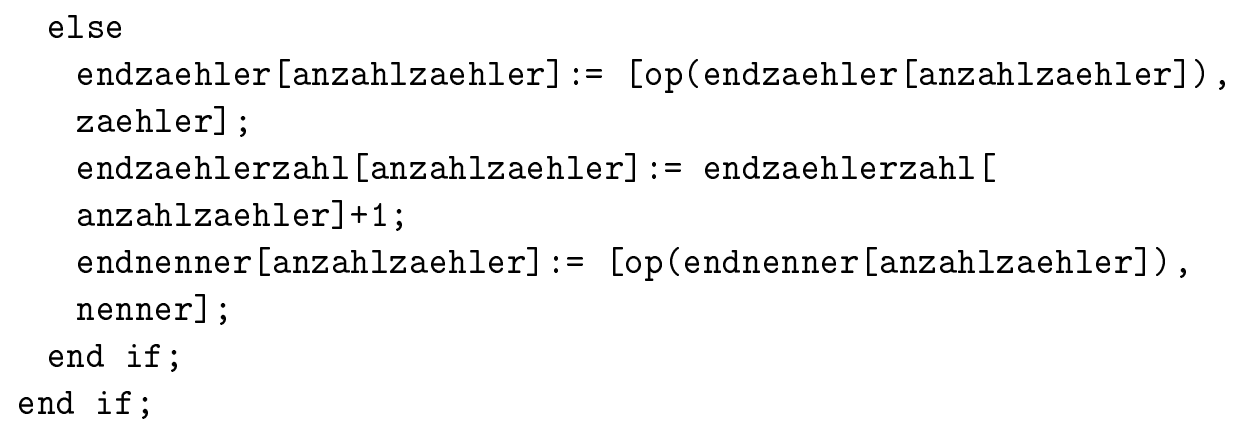

After the for-loop over all ratios of one length is finished, we have to increment the variable for the length anzahlzaehler and bring counter up to date. The lists 
for the resulting invariants and their numbers get their new elements for the next length, if there is a next round of the while-loop. For every length we print the invariants found so far.

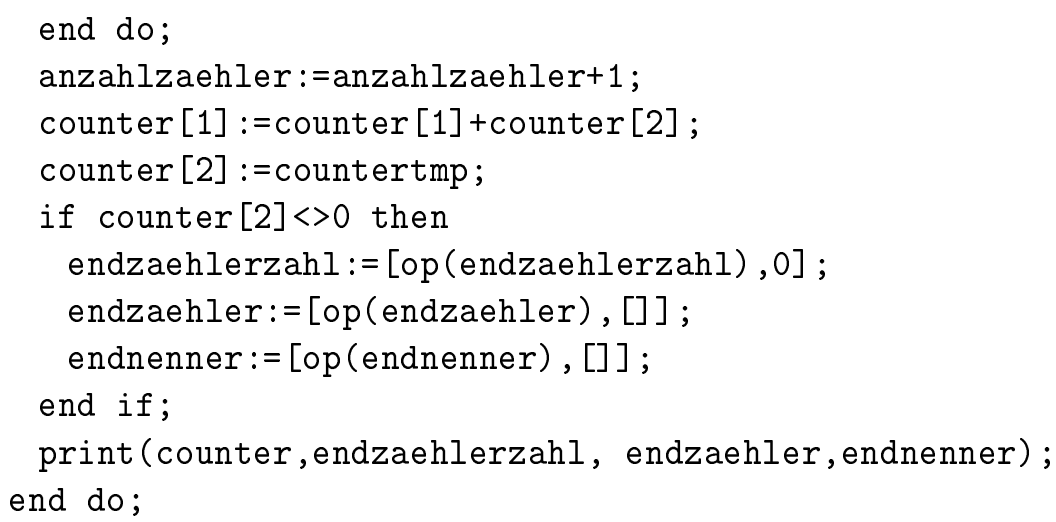

In the fourth round of the while-loop, no ratio is found any more, which can be made longer under the stated conditions. The longest ratios in the lists have four intervals in the numerator and four in the denominator. In the end the 24 trees contain altogether 648 elements and lead to 12 invariants of length 2, 32 of length 3 and 12 of length 4.

They are those of eqns. 4.2.29 4.2 .35 and all possible permutations of indices and can all be expressed by the set of ten invariants, which we choose in sections 4.2 and 4.3 . 


\section{Bibliography}

[AEPS02] G. Arutyunov, B. Eden, A. C. Petkou, and E. Sokatchev. Exceptional non-renormalization properties and OPE analysis of chiral four-point functions in N = 4 SYM(4). Nucl. Phys., B620:380-404, 2002, hepth/0103230.

[AMS76] B. L. Aneva, S. G. Mikhov, and D. Ts. Stoyanov. Some representations of the conformal superalgebra. Theoretical and Mathematical Physics, 27:502-508, 1976. 10.1007/BF01028617.

[AMS77] B. L. Aneva, C. G. Mikhov, and D. Ts. Stoyanov. Properties of representations of the conformal superalgebra. Theoretical and Mathematical Physics, 31:394-402, 1977. 10.1007/BF01036669.

[AMS78] B. L. Aneva, S. G. Mikhov, and D. Ts. Stoyanov. Two- and threepoint functions of conformal superfields. Theoretical and Mathematical Physics, 35:383-390, 1978. 10.1007/BF01039108.

[BIWW05] Edwin Barnes, Kenneth A. Intriligator, Brian Wecht, and Jason Wright. $\mathrm{N}=1$ RG flows, product groups, and a-maximization. Nucl. Phys., B716:33-64, 2005, hep-th/0502049.

[BK95] I.L. Buchbinder and S.M. Kuzenko. Ideas and methods of supersymmetry and supergravity: A Walk through superspace. 1995.

[BMRW09] Marcel Bischoff, Daniel Meise, Karl-Henning Rehren, and Ingo Wagner. Conformal quantum field theory in various dimensions. 2009, 0908.3391. dedicated to Ivan Todorov on the occasion of his 75 th birthday.

[BZ81] L. Baulieu and D. Zwanziger. Equivalence of Stochastic Quantization and the Faddeev-Popov Ansatz. Nucl.Phys., B193:163, 1981.

[CL80] N.H. Christ and T.D. Lee. Operator Ordering and Feynman Rules in Gauge Theories. Phys.Rev., D22:939, 1980.

[DO01a] F. A. Dolan and H. Osborn. Implications of $\mathrm{N}=1$ superconformal symmetry for chiral fields. Nucl. Phys., B593:599-633, 2001, hepth/0006098. 
[DO01b] F.A. Dolan and H. Osborn. Conformal four point functions and the operator product expansion. Nucl.Phys., B599:459-496, 2001, hepth/0011040.

[DO02] F. A. Dolan and H. Osborn. Superconformal symmetry, correlation functions and the operator product expansion. Nucl. Phys., B629:3-73, 2002, hep-th/0112251.

[DO03] F. A. Dolan and H. Osborn. On short and semi-short representations for four dimensional superconformal symmetry. Ann. Phys., 307:41-89, 2003, hep-th/0209056.

[DO04] F. A. Dolan and H. Osborn. Conformal partial waves and the operator product expansion. Nucl. Phys., B678:491-507, 2004, hep-th/0309180.

[DO06] F. A. Dolan and H. Osborn. Conformal partial wave expansions for $\mathrm{N}=4$ chiral four point functions. Annals Phys., 321:581-626, 2006, hep-th/0412335.

[DP85a] V. K. Dobrev and V. B. Petkova. All Positive Energy Unitary Irreducible Representations of Extended Conformal Supersymmetry. Phys. Lett., B162:127-132, 1985.

[DP85b] V. K. Dobrev and V. B. Petkova. On the group theoretical approach to extended conformal supersymmetry: classification of multiplets. Lett. Math. Phys., 9:287-298, 1985.

[DP87] V. K. Dobrev and V. B. Petkova. Group theoretical approach to extended conformal supersymmetry: function space realizations and invariant differential operators. Fortschr. Phys., 35:537, 1987.

[DS74] P.H. Dondi and M. Sohnius. Supergauge Transformations with Isospin Symmetry. Nucl.Phys., B81:317, 1974.

[EF06] J. Earman and D. Fraser. Haag's Theorem and Its Implications for the Foundations of Quantum Field Theory. Erkenntnis, 64:305, 2006.

$\left[\mathrm{EHP}^{+} 00\right]$ B. U. Eden, Paul S. Howe, A. Pickering, E. Sokatchev, and Peter C. West. Four-point functions in $\mathrm{N}=2$ superconformal field theories. Nucl. Phys., B581:523-558, 2000, hep-th/0001138.

[EHSW00] B. U. Eden, Paul S. Howe, E. Sokatchev, and Peter C. West. Extremal and next-to-extremal n-point correlators in four- dimensional SCFT. Phys. Lett., B494:141-147, 2000, hep-th/0004102.

[EHW99] B. Eden, Paul S. Howe, and Peter C. West. Nilpotent invariants in N = 4 SYM. Phys. Lett., B463:19-26, 1999, hep-th/9905085.

$\left[\mathrm{FKL}^{+}\right.$10a] Sergio Ferrara, Renata Kallosh, Andrei Linde, Alessio Marrani, and Antoine Van Proeyen. Jordan Frame Supergravity and Inflation in NMSSM. Phys. Rev., D82:045003, 2010, 1004.0712. 
$\left[\mathrm{FKL}^{+}\right.$10b] Sergio Ferrara, Renata Kallosh, Andrei Linde, Alessio Marrani, and Antoine Van Proeyen. Superconformal Symmetry, NMSSM, and Inflation. $2010,1008.2942$.

[FRS07] Klaus Fredenhagen, Karl-Henning Rehren, and Erhard Seiler. Quantum field theory: Where we are. Lect.Notes Phys., 721:61-87, 2007, hepth/0603155.

[Haa55] R. Haag. On quantum field theories. Kong.Dan.Vid.Sel.Mat.Fys.Med., 29N12:1-37, 1955.

[HH03] P. J. Heslop and P. S. Howe. Four-point functions in N = 4 SYM. JHEP, 01:043, 2003, hep-th/0211252.

[HH04] P. J. Heslop and P. S. Howe. Aspects of N = 4 SYM. JHEP, 01:058, 2004, hep-th/0307210.

[Hil] David Hilbert. Mathematical problems. Bull. Amer. Math. Soc., 8:437479 .

[HLS75] Rudolf Haag, Jan T. Lopuszanski, and Martin Sohnius. All Possible Generators of Supersymmetries of the s Matrix. Nucl.Phys., B88:257, 1975.

[HSSW00] Paul S. Howe, C. Schubert, E. Sokatchev, and Peter C. West. Explicit construction of nilpotent covariants in $\mathrm{N}=4$ SYM. Nucl. Phys., B571:71-90, 2000, hep-th/9910011.

[HSW98] Paul S. Howe, E. Sokatchev, and Peter C. West. 3-point functions in N = 4 Yang-Mills. Phys. Lett., B444:341-351, 1998, hep-th/9808162.

[HW96a] Paul S. Howe and Peter C. West. Is N = 4 Yang-Mills theory soluble? 1996, hep-th/9611074.

[HW96b] Paul S. Howe and Peter C. West. Operator product expansions in fourdimensional superconformal field theories. Phys. Lett., B389:273-279, 1996, hep-th/9607060.

[HW97] Paul S. Howe and Peter C. West. Superconformal Ward identities and $\mathrm{N}=2$ Yang-Mills theory. Nucl. Phys., B486:425-442, 1997, hepth/9607239.

[HW99] Paul S. Howe and Peter C. West. Non-perturbative Green's functions in theories with extended superconformal symmetry. Int. J. Mod. Phys., A14:2659-2674, 1999, hep-th/9509140.

[IW03] Kenneth A. Intriligator and Brian Wecht. The exact superconformal R-symmetry maximizes a. Nucl. Phys., B667:183-200, 2003, hepth/0304128. 
[IW04] Kenneth A. Intriligator and Brian Wecht. RG fixed points and flows in SQCD with adjoints. Nucl. Phys., B677:223-272, 2004, hep-th/0309201.

[JO00] Palle E.T. Jorgensen and Gestur Olafsson. Osterwalder-Schrader axioms - Wightman axioms. 2000, math-ph/0001010.

[Knu11] Holger Knuth. On invariants and scalar chiral correlation functions in $\mathrm{N}=1$ superconformal field theories. Int.J.Mod.Phys., A26:2007-2025, 2011, 1010.2740 .

[LSZ55] H. Lehmann, K. Symanzik, and W. Zimmermann. On the formulation of quantized field theories. Nuovo Cim., 1:205-225, 1955.

[Mac77] G. Mack. All Unitary Ray Representations of the Conformal Group SU(2,2) with Positive Energy. Commun.Math.Phys., 55:1, 1977.

[MPS76] V. V. Molotkov, S. G. Petrova, and D. Ts. Stoyanov. Representations of superconformal algebra. two-point and three-point green's functions of scalar superfields. Theoretical and Mathematical Physics, 26:125-131, 1976. 10.1007/BF01079417.

[NRT05] N. M. Nikolov, Karl-Henning Rehren, and I. T. Todorov. Partial wave expansion and Wightman positivity in conformal field theory. Nucl. Phys., B722:266-296, 2005, hep-th/0504146.

[NRT08] Nikolay M. Nikolov, Karl-Henning Rehren, and Ivan Todorov. Harmonic bilocal fields generated by globally conformal invariant scalar fields. Commun.Math.Phys., 279:225-250, 2008, 0704.1960.

[NRW11] Christoph Neumann, Karl-Henning Rehren, and Lena Wallenhorst. New methods in conformal partial wave analysis. 2011, 1112.3512.

[NST02a] Nikolay M. Nikolov, Yassen S. Stanev, and Ivan T. Todorov. Fourdimensional CFT models with rational correlation functions. J.Phys.A, A35:2985-3008, 2002, hep-th/0110230.

[NST02b] Nikolay M. Nikolov, Yassen S. Stanev, and Ivan T. Todorov. Global conformal invariance and bilocal fields with rational correlation functions. 2002, hep-th/0211106.

[NST03] Nikolay M. Nikolov, Yassen S. Stanev, and Ivan T. Todorov. Globally conformal invariant gauge field theory with rational correlation functions. Nucl.Phys., B670:373-400, 2003, hep-th/0305200.

[NT01] Nikolay M. Nikolov and Ivan T. Todorov. Rationality of conformally invariant local correlation functions on compactified Minkowski space. Commun. Math. Phys., 218:417-436, 2001, hep-th/0009004.

[OS73] Konrad Osterwalder and Robert Schrader. Axioms for euclidean Green's functions. Commun.Math.Phys., 31:83-112, 1973. 
[Osb99] Hugh Osborn. $\mathrm{N}=1$ superconformal symmetry in four-dimensional quantum field theory. Annals Phys., 272:243-294, 1999, hep-th/9808041.

[Par98] Jeong-Hyuck Park. N = 1 superconformal symmetry in 4-dimensions. Int. J. Mod. Phys., A13:1743-1772, 1998, hep-th/9703191.

[Par99] Jeong-Hyuck Park. Superconformal symmetry and correlation functions. Nucl. Phys., B559:455-501, 1999, hep-th/9903230.

[PSD11] David Poland and David Simmons-Duffin. Bounds on 4D Conformal and Superconformal Field Theories. JHEP, 1105:017, 2011, 1009.2087.

[QKS10] Fernando Quevedo, Sven Krippendorf, and Oliver Schlotterer. Cambridge Lectures on Supersymmetry and Extra Dimensions. 2010, 1011.1491.

[Sei95] N. Seiberg. Electric - magnetic duality in supersymmetric nonAbelian gauge theories. Nucl. Phys., B435:129-146, 1995, hep-th/9411149.

[Sha03] Nausheen R. Shah. Minimal Supersymmetric Standard Model (MSSM). 2003, http://theory.uchicago.edu/ sethi/Teaching/P487S2003/MSSMnausheen.pdf.

[Soh85] M.F. Sohnius. Introducing Supersymmetry. Phys.Rept., 128:39-204, 1985.

[SW] R.F. Streater and A.S. Wightman. PCT, spin and statistics, and all that. Benjamin, 1964; Princeton Univ. Press, Princeton, N.J.

[Wag] I Wagner. Twist-2-Partialwellen höherer Korrelationsfunktionen in global konform-invarianter Quantenfeldtheorie. Diploma thesis.

[Wig39] Eugene P. Wigner. On Unitary Representations of the Inhomogeneous Lorentz Group. Annals Math., 40:149-204, 1939.

[WZ74] J. Wess and B. Zumino. Supergauge Transformations in FourDimensions. Nucl.Phys., B70:39-50, 1974.

[Zwa81] Daniel Zwanziger. Covariant Quantization of Gauge Fields Without Gribov Ambiguity. Nucl.Phys., B192:259, 1981. 


\section{Acknowledgements}

I would like to thank Prof. K.-H. Rehren for suggesting a very interesting topic, which I have had the possibility to explore for the last years and which let me pass quite a few turning points on the way to the goal. It allowed me not only to dwell on a really novel intersection of supersymmetry and the Wightman axiomatic approach, but also to be concerned with computer algebraic methods, which I enjoyed and which helped me to understand essential connections. The discussions with him have always been very fruitful concerning details as well as the general view. Major parts of the organization of the always inspiring LQP workshops lie also on his shoulders, when it takes place in Göttingen once a year, for which I want to thank him, as well.

I also would like to express my gratitude to Prof. L. Covi, who accepted on relatively short notice to be the second examiner for this dissertation.

Thank you also to Prof. D. Buchholz, who has been my second adviser and supported me for my application for the graduate scholarship of the Evangelisches Studienwerk e.V. Villigst and the three successful requests of extension. I have been supported by this scholarship from November 2007 until October 2010.

Furthermore I owe my gratitude to Prof. R. Meyer for his work as speaker of the Research Training Group 1493 "Mathematische Strukturen in der modernen Quantenphysik", especially for the great project of the "DoktorandInnenzeitung" and the suggestion, that I could finish my thesis supported by a short scholarship of this RTG from October until December 2011. I also thank his designated successor Prof. D. Bahns for her commitment for the continuation of this RTG.

It has also been a great time at the institute with many interesting discussions, for which I would like to thank specifically the ones, with whom I shared the office, Antonia Kukhtina, Daniela Cadamuro and in the last months also Gennaro Tedesco.

I am deeply grateful to my parents for all they have done for me in my life, which brought me here finishing my dissertation.

My greatest thanks go to my wife, Christina, who kept me grounded over difficult periods of my work in the last years, although her patience has been tested excessively in that time. 



\title{
Lebenslauf
}

\author{
Holger Knuth \\ Lister Kirchweg 29 \\ 30163 Hannover \\ Tel. 0511-7690196 \\ knuth@theorie.physik.uni-goettingen.de
}

Geboren am 19.06.1981 in Bergisch-Gladbach

Deutsche Staatsangehörigkeit

\begin{tabular}{|c|c|}
\hline Juni 2000 & Abitur am Maximilian-Kolbe-Gymnasium, Köln-Porz-Wahn \\
\hline September 2001 & Studium der Physik an der Rheinischen Friedrich-Wilhelms- \\
\hline bis Dezember 2006 & Universität Bonn \\
\hline September 2003 & Diplom-Vorprüfung \\
\hline $\begin{array}{r}\text { September } 2004 \\
\text { bis Juni } 2005\end{array}$ & $\begin{array}{l}\text { Studienaufenthalt im Rahmen des Sokrates/Erasmus Programms } \\
\text { an der Universiteit van Amsterdam }\end{array}$ \\
\hline Dezember 2006 & $\begin{array}{l}\text { Diplomprüfung. Titel der Diplomarbeit: } \\
\text { „Fusion Algebras and Verlinde-Formula in Logarithmic } \\
\text { Conformal Field Theories”. } \\
\text { Anleitung: Priv.-Doz. Dr. Michael Flohr }\end{array}$ \\
\hline Seit September 2007 & $\begin{array}{l}\text { Bearbeitung des Promotionsthemas } \\
\text { „Superconformal Invariants and Correlation Functions” } \\
\text { unter Betreuung von Prof. Dr. Karl-Henning Rehren }\end{array}$ \\
\hline
\end{tabular}

